\title{
Characterization of Novel Optoelectronic Semiconductor Materials and Devices
}

\author{
A Dissertation \\ Presented to \\ the faculty of the School of Engineering and Applied Science \\ University of Virginia \\ in partial fulfillment \\ of the requirements for the degree of \\ Doctor of Philosophy
}

By

Lijun Li

December

2014 
APPROVAL SHEET

The dissertation

is submitted in partial fulfillment of the requirements

for the degree of

Doctor of Philosophy

AUTHOR

The dissertation has been read and approved by the examining committee:

Joe C. Campbell

Advisor

Robert M. Weikle II

Stephen G. Wilson

Lloyd R. Harriott

Lin Pu

Accepted for the School of Engineering and Applied Science:

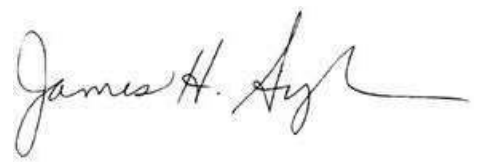

Dean, School of Engineering and Applied Science

December

2014 
This dissertation is dedicated to my mother, Manling, for her unconditional love. 
"Education is the path from cocky ignorance to miserable uncertainty."

--- Mark Twain 


\begin{abstract}
The continuous arrival of novel optoelectronic semiconductor materials and devices in recent years has resulted in a demand for adequate metrology to extract important physical parameters and metrics as well as understanding the fundamentals of material and device physics. This dissertation is dedicated to the development and/or application of four characterization methods. The transient photocurrent method is used to measure mobilities and lifetimes of materials that are used to fabricate organic solar cells, which are very crucial physical parameters related to the device performance. The photoinduced current transient spectroscopy technique is applied for the first time to hybrid solar cells in order to extract the trap density of states, which is not easily accessible otherwise. Low-frequency noise spectroscopy near DC is developed and applied to organic solar cells and high-power photodiodes; the results are correlated with charge conduction processes. Finally, a UHF-band RF system based on resonance-coupled photoconductivity decay for the purpose of contactless measurement of carrier recombination lifetimes in semiconductor wafers is built, and is further improved with in-phase and quadrature (IQ) mixing. All four methods have shown great potential and importance in addressing the new metrological challenges from novel optoelectronic materials and devices. I conclude with proposals for future work related to noise measurement and contactless characterization.
\end{abstract}




\section{Acknowledgments}

I would like to take this opportunity to give my appreciation to all the people who have helped make who I am. First of all, I am very fortunate to have Prof. Joe Campbell, a renowned researcher with Hall-of-Fame caliber, as my advisor. With his signature insights and immeasurable patience, he has been guiding all the students in the group to fledge. I have no doubt that what I learned from Prof. Campbell is going to yield lasting dividends in the coming years. Secondly, this dissertation will not be possible without the undiscounted support from my other PhD committee members Prof. Bobby Weikle, Prof. Stephen Wilson, Prof. Lloyd Harriott and Prof. Lin $\mathrm{Pu}$, who have been providing precious suggestions and generous help. A big Danke also goes to Prof. Andreas Beling whom I am very lucky to befriend for years, for patiently answering every question from me, sometimes a little off the line, with the unadulterated German caliber. By no means should not I thank Dr. Xiaoguang Zheng, a mentor and friend in Campbell's group, for not only the unselfish help on my research and career, but also the sharing of the excitement of being in this era. I sincerely appreciate the crucial support I received from Prof. Avik Ghosh, Prof. Mircea Stan, Prof. Keith Williams and Prof. Nathan Swami in my early years at the University. Besides, I am fortunate to work with our collaborators at the University of Southern California. The study on the novel organic and hybrid solar cells will not be possible without the generous support from Prof. Barry Thompson, Petr Khlyabich and Beate Burkhart (organic solar cells), and also Prof. Richard Brutchey and Matt Greaney (hybrid solar cells).

Besides, I received numerous help from other faculty members, staff and graduate students in the department. I am always very grateful for the unreserved knowledge transfer from Prof. Harry Powell, the most knowledgeable person I have known on the electronic circuits and embedded systems. I must thank Prof. Scott Barker for the enlightening discussion on RF circuits. I enjoyed every minute of talking with Harry Wade for sharing the enjoyable pieces in and out of UVA's cleanroom. Also, I would like to thank the essential support and patience from Prof. Arthur Lichtenberger, Joe Beatrice, Alex Lobo, Tim Pernell, Jian Zhang, Michael Stogoski, Jie Wang, Michael Cyberey, Naser Alijabbari, Lihan Chen and Qiang Yu in the cleanroom. I cherish my friendship with Eileen Thacker, the former Graduate Coordinator of the department who is now probably joyfully playing mandolin in the scenic countryside, and thank her for the encouragement and help in my early years here. I also would like to sincerely acknowledge the 
assistance from Yadira Weaver, Joan Sipe, Susan Malone, Daniel Fekto and Gloria Walker. Last but not least, I fully enjoyed and appreciated the fellowship with Frank Tseng, Dincer Unluer, Kamaram Munira, Medhi Kabir, Carlos Polanco, Qian Sang and Yanqing Zhang when I was still sitting in the second floor of Thornton Hall C-wing years ago.

All students in Campbell's group are essentially my siblings with different last names. The positive group chemistry has been an indispensable source of inspiration and resilience in research. Especially, I should thank Kejia Li and Yang Shen for their help on the solar cell project. The research maturity I learned from them lightened my way for quality work. I cannot forget the help from Qiugui Zhou and Dion McIntosh when I first joined the group. Moreover, I have been extremely fortunate to bask in the comradeship with Yang Fu, Zhi Li, Huapu Pan, Joe Murray, Wenlu Sun, Zhiwen Lu, Yaojia Chen, Jinrong Yuan, Baile Chen, Angad Sachdeva, Min Ren, Xiaojun Xie, Qinglong Li, Zhanyu Yang, Maddy Woodson, Ye Wang, Chenfei Jin and our mascot Jean (a daydreaming Siberian Husky who doesn't love?). My graduate school life is gleefully colored by their versatile personalities and the good time spent together consisting of group lunching, hiking, running, golfing, footballing, frisbeeing and watching the games of UVA men's basketball team coached by Tony Bennett.

Finally I would like to dedicate this big moment to honoring my parents, Manling Shou and Fudong Li. Without the unconditional support from my mother in the past decade, I would not have knocked on the door of the graduate school. And my dear father, a seasoned and intelligent mechanic engineer who planted the technological seeds inside me when I was a child, will surely take a moment to sip from a glass of wine in the heaven. It was also my great pleasure to meet Yiqi Huang in the beautiful Charlottesville, with whom I shared our lives and fledged together in the past few years.

It has been a six-year uneven but rewarding journey. I believe it will be invaluable assets for the rest of my life. 


\section{Table of Contents}

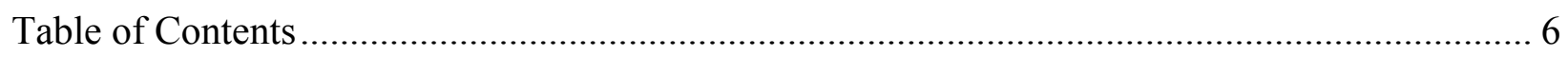

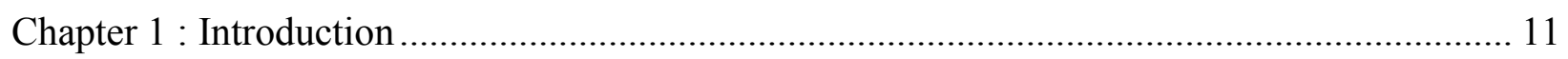

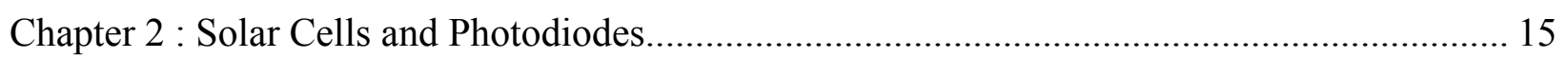

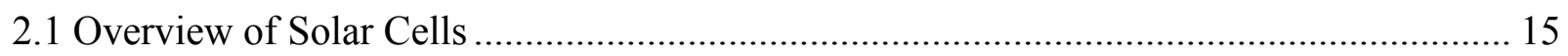

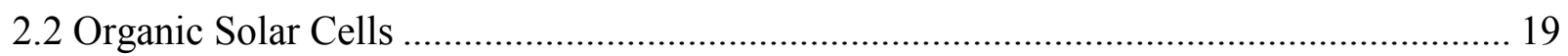

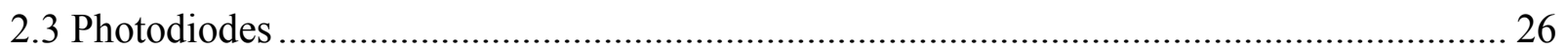

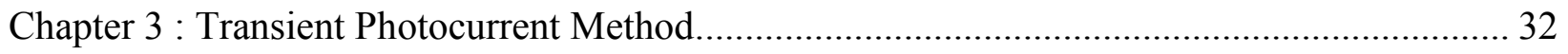

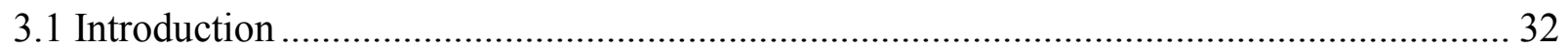

3.2 Transient Photoconductivity for Organic Solar Cells ...................................................... 34

3.3 Transient Photocurrents of Polymer-Fullerene Bulk Heterojunction Organic Solar Cells. 36

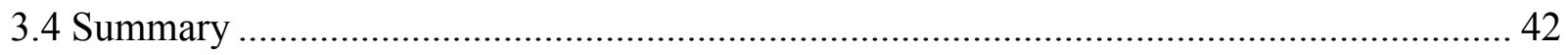

Chapter 4 : Photo-induced Current Transient Spectroscopy............................................................ 43

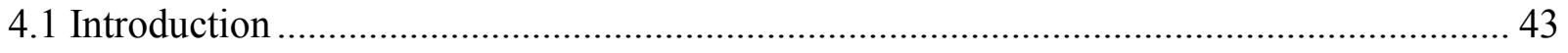

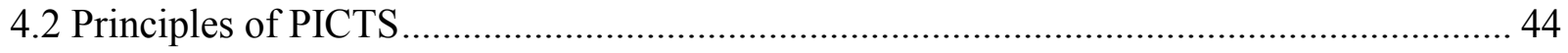

4.3 Data Processing of PICTS......................................................................................... 46

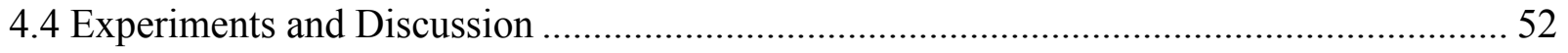

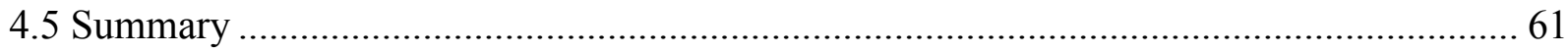

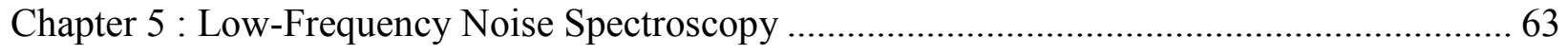

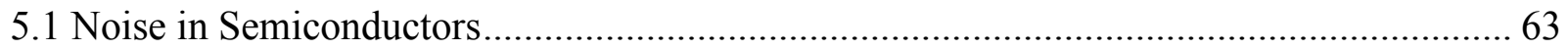

5.2 Low Frequency Noise in Organic Solar Cells..................................................................... 69

5.3 Low-Frequency Noise in MUTC Photodiodes …………................................................ 78

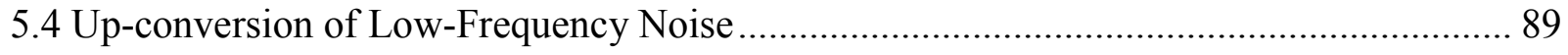

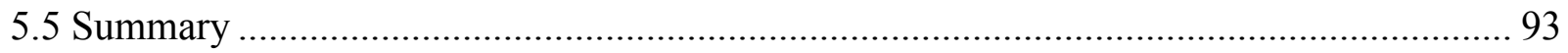

Chapter 6 : Resonance-Coupled Photoconductivity Decay Measurement .................................... 95

6.1 Contactless Characterization of Semiconductor Materials ................................................. 95

6.2 Resonance-Coupled Photoconductivity Decay Measurement .............................................. 97

6.3 Improved RCPCD Measurement with IQ Mixing ......................................................... 105

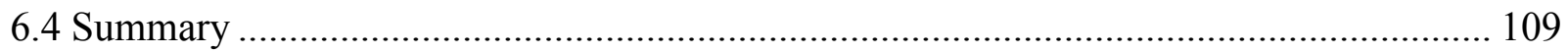

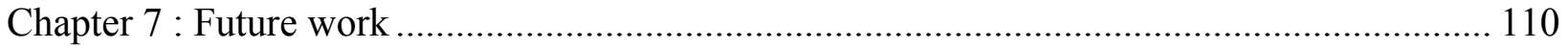

7.1 Phase Noise Measurement of High-Power Photodiodes with Phase-Sensitive Detection 110

7.2 Contactless Characterization of Organic Thin Films ................................................... 113

Appendix: Source Code for PICTS Data Processing ……….................................................... 115

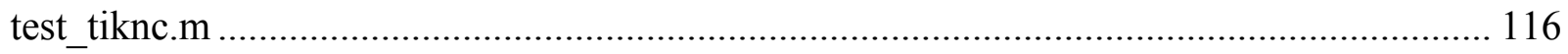




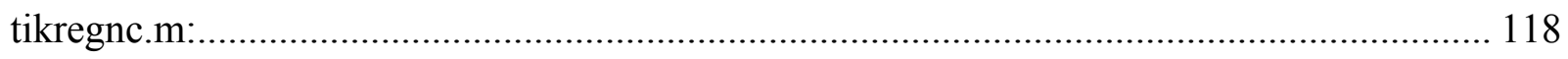

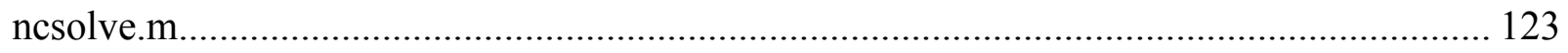

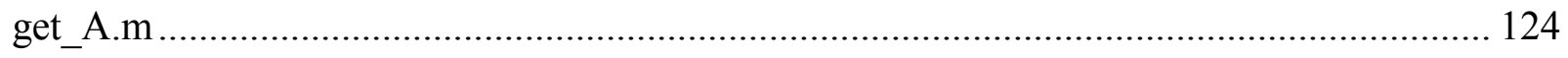

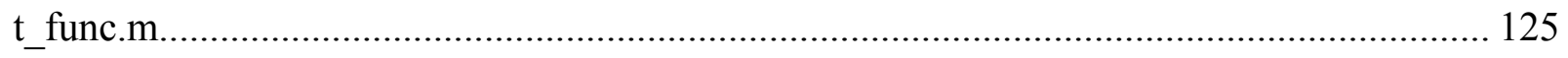

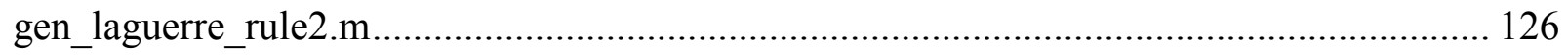

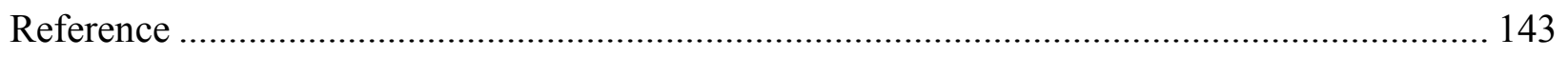




\section{List of Figures}

Figure 1.1: Various applications and products powered by semiconductor devices. Upper left: iPhone from Apple. Middle left: solar panels under the sun. Lower left: Smart thermostat from Nest Labs (acquired by Google in 2014) for home automation. Upper right: a home theater with a large LED display. Lower right: Google's self-driving car (2014 model). .............................. 12 Figure 2.1: An illustration of working principles of a typical pn-junction inorganic solar cell. $\mathrm{E}_{\mathrm{bi}}$

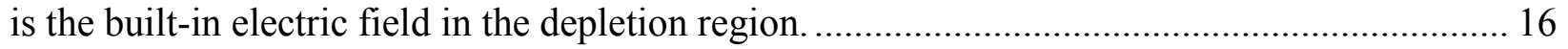
Figure 2.2: Typical current-voltage characteristics of a solar cell in dark and illuminated conditions. Important parameters, such as open-circuit voltage $\mathrm{V}_{\mathrm{oc}}$, short-circuit current $\mathrm{I}_{\mathrm{sc}}$, and maximum deliverable power $P_{\max }$, are labeled on the plot. Fill factor can be calculated from those three parameters. $\mathrm{V}_{0}$ and $\mathrm{I}_{0}$ are the voltage and current corresponding to the maximum power point.

Figure 2.3: Three different standard solar spectra. The AM1.5 Global (AM1.5G) spectrum is for the terrestrial use of flat photovoltaic cells and modules with power density of $1000 \mathrm{~W} / \mathrm{m}^{2}$ or 100 $\mathrm{mW} / \mathrm{cm}^{2}$. The AM1.5 Direct (AM1.5D) is for the terrestrial use of solar concentrators. The AM0 spectrum is for the photovoltaic applications in space. Usually, AM1.5G is most widely used for lab cells. This figure is from Ref [24], and the original data can be found in Ref[25]................ 19 Figure 2.4: Best power conversion efficiencies of different PV cells certified by National Renewable Energy Laboratory (Source: Ref [26]) ……………………………………….... 20 Figure 2.5: The device structures and light harvesting processes of (a) bilayer and (b) bulk heterojunction organic solar cells. The pink dots in the magnified circles stand for small molecule acceptors like PCBM, while the wiggling chains in the other magnified circles

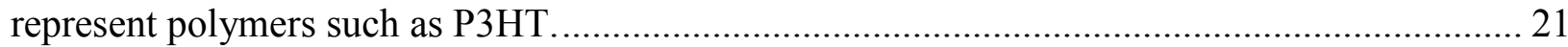

Figure 2.6: The energy band diagram of P3HT:PCBM organic solar cells............................... 22 Figure 2.7: The light harvesting processes of bulk heterojunction organic solar cells................. 24 Figure 2.8: Step-by-step fabrication process of typical P3HT:PCBM bulk heterojunction organic solar cells ..................................................................................................................... 25

Figure 2.9: P3HT:PCBM bulk heterojunction solar cells fabricated in our lab. There are eight

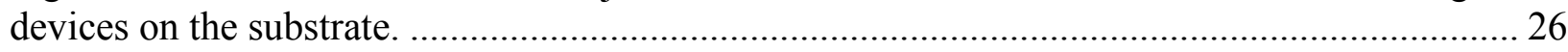

Figure 2.10: An illustration of a p-n junction photodiode in working condition......................... 27 Figure 2.11: An illustration of the figures of merit for photodiode linearity. The image is based on the class notes of ECE6261 Microwave Engineering II taught by Bobby Weikle at the University of Virginia.

Figure 2.12: The (a) band diagram and (b) device structure of a MUTC1 photodiode. The image is from Ref[33] ....................................................................................................... 31 Figure 3.1: (a) The experimental concept of transient photoconductivity and (b) the typical transient photocurrent in an inorganic semiconductor where both electrons and holes participate in the charge transport. The orange solid line is the transient photocurrent without diffusion, while the red solid line is the transient photocurrent with the diffusion......................................... 35 Figure 3.2: (a) Device structure of P3HT:PCBM and P3HTT-TP:PCBM bulk heterojunction organic solar cells. (b) The measurement circuit for transient photocurrents................................ 39 Figure 3.3: The normalized transient photocurrents of (a)P3HT:PCBM and (b)P3HTT-TP:PCBM BHJ organic solar cells excited by $640 \mathrm{~nm}$ light pulses. J is the photocurrent density. Both devices are measured under 1 sun equivalence. 
Figure 3.4: Transient photocurrent decay under $-2 \mathrm{~V}$ reverse bias for (a) P3HT:PCBM and (b) P3HTT-TP:PCBM organic solar cells. The photocurrent is normalized by its initial value at $\mathrm{t}=0$.

Figure 3.5: Transient photocurrent decay time constant $\tau_{1 / \mathrm{e}}$ as a function of the forward applied

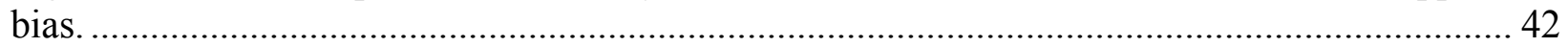

Figure 4.1: The trapping model in semiconductors for PICTS method................................... 45

Figure 4.2: The PICTS data processing workflow............................................................. 47

Figure 4.3: A graphical demonstration of the principle of L-curve criterion. $\lambda$ is the regularization

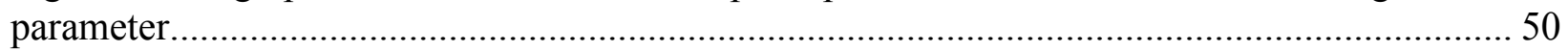

Figure 4.4: A graphical demonstration of 5-fold ordinary cross-validation for PICTS data

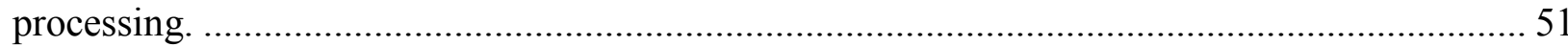

Figure 4.5: (a) The transient current corresponding to the artificially generated spectrum in Table 4.1but with additive white noise. (b) The solution to the inverse transform of the transient current in (a) with 200 sampling points in f-space. 52 Figure 4.6: (a) Typical PICTS transient photocurrents; (b) typical trap emission spectra after the inverse transform of transients; (c) Arrhenius plot of P3HT:CdSe(tBT); (d) Arrhenius plot of

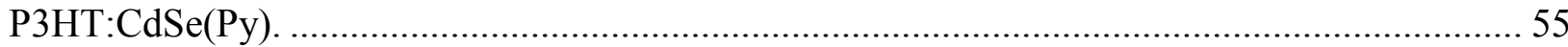
Figure 4.7: (a) Trap emission strength and (b) energetic broadening ( $\boldsymbol{\sigma} \boldsymbol{E T})$ of P3HT:CdSe(tBT)

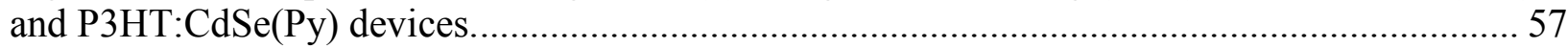
Figure 4.8: The visualization of the trap parameters in Table 4.3 and Figure 4.7.................... 59 Figure 4.9: (a) Fast photocurrent transient decay normalized to the peak values for P3HT:CdSe(tBT) and P3HT:CdSe(Py); (b) Log-log plots providing a quantitative value of the transit times $\left(\mathrm{t}_{\mathrm{tr}}\right)$............................................................................................... 59 Figure 4.10: TEM images of (a) P3HT:CdSe(Py) and (b) P3HT:CdSe(tBT) BHJ films. .......... 61 Figure 5.1: (a) Thevenin and (b) Norton representation of noise sources. ................................ 64 Figure 5.2: Carrier fluctuation caused by the trapping and detrapping in (a) a MOSFET and (b) a diode. 67

Figure 5.3: The illustration of how G-R noise resulted from multiple defects can give rise to "1/f" signature. 68

Figure 5.4: The circuit diagram for the low-frequency noise measurement. The bias on the devices under test is applied from the SR570 amplifier. C1 and R1 make a high-pass filter with

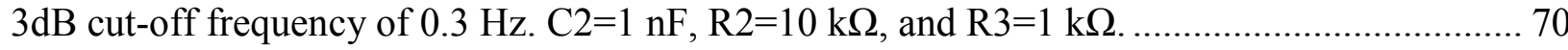
Figure 5.5: The dark current-voltage characteristics of four P3HT:PCBM devices annealed at 60 ${ }^{\circ} \mathrm{C}, 95^{\circ} \mathrm{C}, 110^{\circ} \mathrm{C}$ and $140{ }^{\circ} \mathrm{C}$. 72 Figure 5.6: Four LFN spectrum examples under forward bias. The system noise is tested with an $1 \mathrm{M}$ ohm resistor. The measured " $1 / \mathrm{f}$ " slope is around 1.5 . 73 Figure 5.7: Current noise power spectral density at $10 \mathrm{~Hz}\left(\mathrm{~S}_{\mathrm{I} 0}\right)$ versus forward and reverse DC dark currents of devices annealed at different temperatures 74 Figure 5.8: The ratio of the Hooge parameter to the carrier recombination lifetime as a function of the annealing temperatures. All values are calculated at $\mathrm{I}_{\mathrm{DC}}=1 \times 10^{-7} \mathrm{~A}$ in the forward bias. . 78 Figure 5.9: The dark low-frequency current noise power spectral density at $10 \mathrm{~Hz}$ of MUTC4 photodiodes with different active area sizes in the (a) forward and (b) reverse bias conditions. The dashed-lines indicate the fitted $\gamma$ value, the power law relationship between the current noise

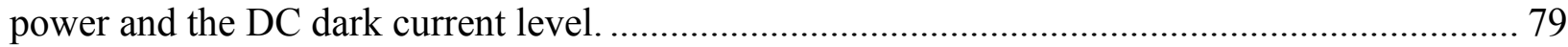
Figure 5.10: Photonoise measurement with balanced photodiodes...................................... 82 
Figure 5.11: Multiple repetitive measurements of (a) dark noise and (b) photonoise (DC photocurrent $\sim 1 \mathrm{~mA}$ ) of commercial photodiodes from AOI under $-5 \mathrm{~V}$ reverse bias.

Figure 5.12: The photonoise of AOI commercial photodiodes with DC photocurrent levels of (a) $0.1 \mathrm{~mA}$, (b) $1 \mathrm{~mA}$ and (c) $7 \mathrm{~mA}$ under reverse bias of $-1 \mathrm{~V},-3 \mathrm{~V},-5 \mathrm{~V}$ and $-7 \mathrm{~V}$. For (c), only data under reverse bias of $-1 \mathrm{~V}$ is measured because of the unstable current under higher reverse bias. Also, the noise floor begins rolling off above $1 \mathrm{kHz}$ because of the bandwidth limit of the

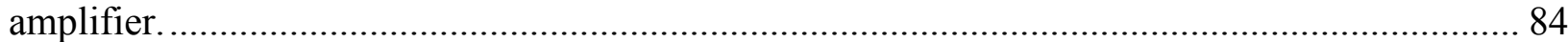

Figure 5.13: Packaged MUTC4 photodiodes from Finisar.................................................... 85 Figure 5.14: The photo-LFN of packaged MUTC4 photodiodes under (a) 0V and (b) -4V bias. 85 Figure 5.15: Photonoise PSD at $10 \mathrm{~Hz}$ versus averaged photocurrent level for MUTC4 photodiodes. 86

Figure 5.16: The illustration of (a) a highly-doped p-n junction (b) band-to-band tunneling and (c) trap-assisted tunneling. The tunneling barrier height $\mathrm{E}_{\mathrm{bar}}$ usually equals the band gap $\mathrm{E}_{\mathrm{g}}$ for a homojunction diode. 88

Figure 5.17: The electric field profiles of a typical MUTC4 photodiode under different illumination levels. Note that for a $40 \mu \mathrm{m}$-diameter MUTC4 photodiode, the $4.8 \mathrm{kA} / \mathrm{cm} 2$ current density corresponds to $60 \mathrm{~mA}$ photocurrent. This figure is from Ref[33]............................. 88 Figure 5.18: The photocurrent $\mathrm{I}_{\mathrm{ph}}$ (black solid line) and the mean of responsivity $\mathrm{R}$ (orange solid line) as a function of input optical power $\mathrm{P}_{\text {in }}$ in a typical photodiode. The $\mathrm{I}_{\mathrm{ph} \text {,sat }}$ is the saturation photocurrent. $\mathrm{R}_{0}$ is the small-signal responsivity. $\mathrm{P}_{\mathrm{a}}$ and $\mathrm{P}_{\mathrm{b}}$ are the operating points in the linear region and non-linear region. 90

Figure 6.1: A typical experimental setup for time-resolved microwave conductivity measurement. The image is from Ref [161].

Figure 6.2: The circuit block diagram of RCPCD measurement. The impedance of the contactless load is subject to change upon the photoexcitation. 98

Figure 6.3: The RCPCD setup with a single mixer.

Figure 6.4: An illustration of the solenoid with a dielectric slab (e.g. silicon wafer) on the top in the RCPCD resonance circuit.

Figure 6.5: The driving and measurement circuits of the pulsed light source, and the photocurrent response of the (a) complete pulse, (b) the falling edge of the pulse and (c) the rising edge of the pulse.

Figure 6.6: (a) The absolute and (b) normalized transient RF response induced by the conductivity of the high-resistivity silicon wafer at different input RF power levels. The DC offsets are all subtracted already.

Figure 6.7: The circuit block diagram of improved RCPCD measurement with IQ mixers. The part inside the dashed circle lines is the implementation of IQ mixers using discrete components.

Figure 6.8: The transient response of (a) I and Q channels and (b) the extracted amplitude and phase measured by the improved RCPCD method with IQ mixing. 108 Figure 7.1: (a) Principle of optics-based microwave signal generation. Part of the figure is from Ref[33]. (b) A simplified block diagram of photodiode phase noise measurement. ................ 112 Figure 7.2: AM-PM conversion factor measurement. This figure is from Ref[166]................ 112 Figure 7.3: The improved resonance circuit. .............................................................. 114 


\section{Chapter 1 : Introduction}

Since the advent of the industrialization of semiconductors in the $20^{\text {th }}$ century, pioneering products made from semiconductor materials and devices that were unthinkable by the previous generations have drastically changed the lives of essentially all humans on the planet. In the dawn of the "third great wave" [1], the era of the digital revolution, the impact has been and will continue to be pervasive in all aspects. The semiconductor lasers inside digital media players such as compact disc (CD) and digital versatile disc (DVD) players have reshaped the business of music and multimedia industries. The large-scale production of light emitting diodes (LEDs) has been refreshing the records on the size and image fidelity of displays that influence lives both at work and home. The jarring cost reduction of mass storage and high-speed optoelectronic transceivers in mega-size data centers has enabled "cloud computing" in the $21^{\text {st }}$ century, causing a shift away from local desktops. The commercialization of organic electronics, especially the organic light emitting diodes (OLEDs), is one of the major driving forces behind affordable consumer electronics such as smartphones, tablets, and smart watches. All of this has not only given rise to multiple multinational billion-dollar businesses, but has also changed the way people communicate with others and perceive their surroundings. Various sensors made from semiconductor devices and circuits, which have been the powering advanced robots in the past, will alter transportation (e.g., autonomous driving), manufacturing, and health care in unprecedented scales. These sensors will also become the backbones of the Internet of Things that will penetrate into previously unreachable corners, possibly establishing a network of 50 billion interconnected devices by 2020 as predicted by Cisco [2]. Last but not least, semiconductor photovoltaic technology is actively participating in the exploration of renewable energy generation, which is crucial for sustainable development of the human society in the foreseeable future.

Optoelectronic semiconductor devices are the type of semiconductor devices that manipulate both optical and electrical power. They can be semiconductor lasers or LEDs that convert the injected electric current to optical outputs, semiconductor photodetectors (e.g. photodiodes, phototransistors) and solar cells that convert the input optical signal to electric current, or other devices such as optical modulators that do not participate in the energy conversion. They are the indispensable building bricks of optoelectronic modules for various applications, such as fiber 
optical communication [3], photovoltaic energy generation [4], biological agent detection [5], optical image sensing [6], ultrastable microwave signal generation [7], and much more.

Although there have been decades of active research and development on optoelectronic materials and devices, many challenges remain as new applications emerge. For example, there has been a great demand for low-cost narrow linewidth lasers with wide tunability for high-speed interconnects in large data centers [8]. Photodiodes with good photoresponse in deep ultraviolet or far infrared wavelengths are crucial for various types of sensing. High-speed photodiodes with high power handling are also of great interest [7]. Solar cells with higher optical absorption are still aggressively pursued, pushing the power conversion efficiency toward the theoretical limit [9] and even beyond with multiexciton generation [10]. The pursuit of "the better" never ends for optoelectronic devices.

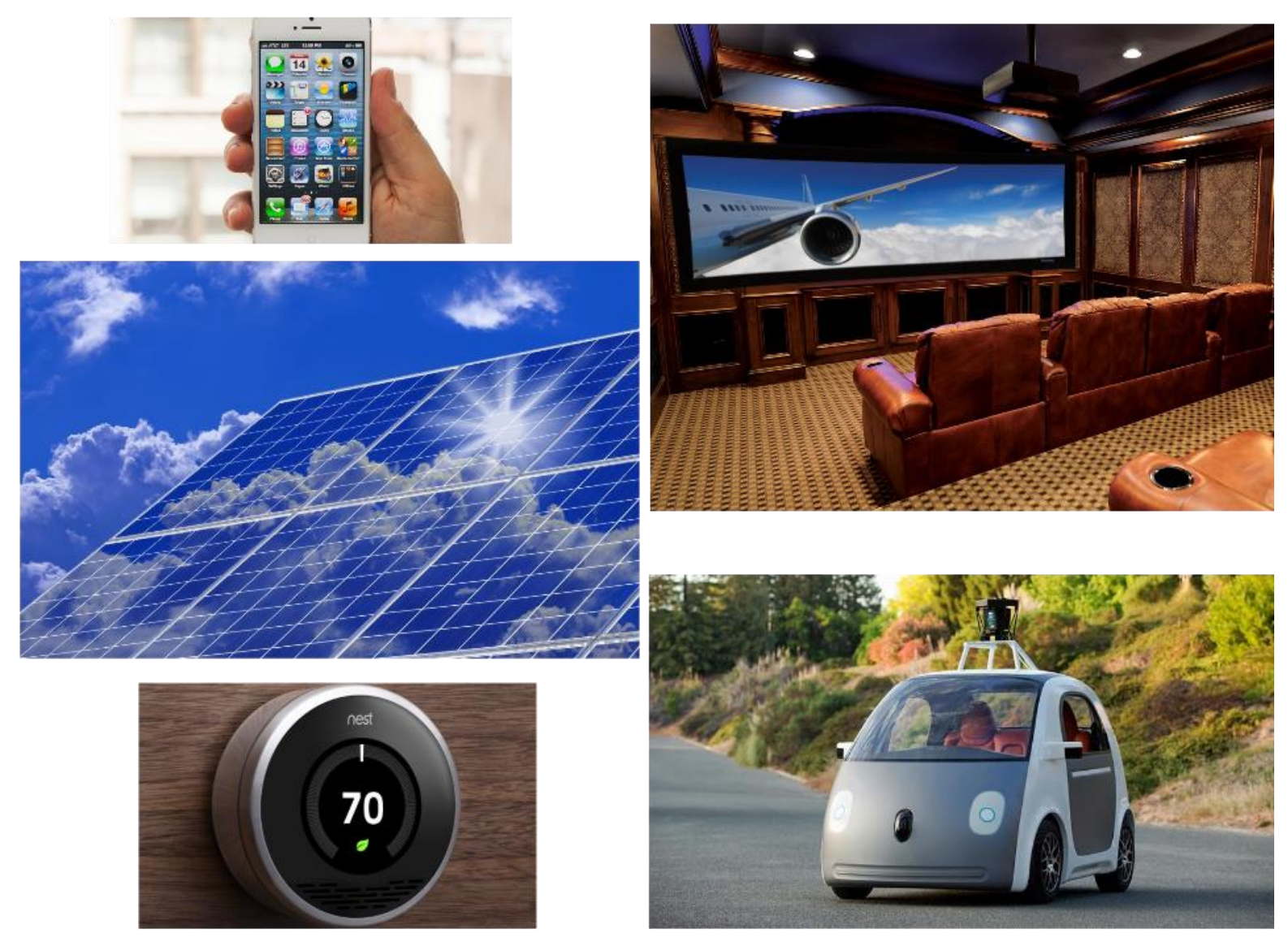

Figure 1.1: Various applications and products powered by semiconductor devices. Upper left: iPhone from Apple. Middle left: solar panels under the sun. Lower left: Smart thermostat 
from Nest Labs (acquired by Google in 2014) for home automation. Upper right: a home theater with a large LED display. Lower right: Google's self-driving car (2014 model).

On the way to "the better", the difficulties in optoelectronic material growth and device fabrication are not the only challenges. Physical characterization after the design and fabrication is an essential step to access qualitative or quantitative physical parameters. It is usually challenging to characterize novel optoelectronic materials and devices because:

- The measurement may be limited by absence of requisite technologies. For example, even if a photodiode with $10 \mathrm{THz}$ bandwidth can be fabricated (which is not possible in the current technology), it is still hard to test its response near that high frequency with the current technology.

- Parameter extraction is based on certain physical model, but the model may not be well defined for novel materials and devices and should be reworked. Either the characterization method should be revised for such change, or the data need to be interpreted differently. A well-known example is that the modified energy band theory behind organic electronics is adapted from the crystalline inorganics.

Developing the novel characterization methods for novel materials and devices is worthwhile because:

- Testing previously unreachable parameters, although difficult, is required to validate the design goals.

- The revised physical models need to be verified. This is crucial not only for the understanding of the novel properties, but also for optimization of materials and devices.

- Novel characterization may also probe the physical properties from complementary angles.

This dissertation is dedicated to understanding the properties of novel optoelectronic materials and devices with different characterization methods. Specifically, the transient photocurrent method, photo-induced current transient spectroscopy, low-frequency noise spectroscopy, and resonance-coupled photoconductivity decay are applied to study novel solar cells and photodiodes. The organization is as follows: 
- Chapter 2 provides an overview of solar cells and photodiodes.

- Chapter 3 introduces the transient photocurrent method, and explains how it can be used to study the physics of bulk heterojunction organic solar cells.

- Chapter 4 applies the photo-induced current transient spectroscopy to bulk heterojunction hybrid organic-inorganic solar cells for the first time, and correlate the distribution of trap states to the DC photovoltaic performance.

- Chapter 5 first gives an overview of noise, and then is focused on the low-frequency noise in semiconductor devices. The low-frequency noise is measured on organic solar cells and high-power photodiodes. This is followed by the discussion on its correlation with the underlying charge conduction processes.

- Chapter 6 discusses the contactless characterization of semiconductors using resonance coupled photoconductivity decay measurement. A UHF-band RF system is built and improved to accurately measure the carrier recombination lifetime in a silicon wafer.

- Chapter 7 suggests possible future work. 


\section{Chapter 2 : Solar Cells and Photodiodes}

\subsection{Overview of Solar Cells}

The ascent of the photovoltaic (PV) industry has been prominent in the last decade despite the intermittent influence of economic and political disruptions. PV-generated power continues to be one of the major contenders in the renewable energy market with excellent prospects for years to come. The global cumulative PV capacity exceeded 100 gigawatts by the end of 2012 [11], 12\% of which was in the United States [12]. PV is already the number-one new source of electricity in the European Union, which also expects additional PV capacity of 48 84 gigawatts by 2017 [11]. As a comparison, in 2012, there were 104 nuclear reactors in US, and the average generation capacity of a single reactor was 0.85 gigawatt [13].

The existing PV technologies can be grouped into three categories. The crystalline silicon (either mono-crystalline or multi-crystalline) wafer-based PV technology first developed in mid-late $20^{\text {th }}$ century is referred as the $1^{\text {st }}$ generation PV technology. Thin film PV technology, which includes amorphous silicon, cadmium telluride (CdTe), copper indium gallium selenide (CIGS) or another variant, is referred as the $2^{\text {nd }}$ generation PV technologies. The $3^{\text {rd }}$ generation PV technologies include all other emerging candidates such as dye-sensitized solar cells, organic solar cells, quantum dot solar cells, and organic-inorganic solar cells. While the $3^{\text {rd }}$ generation PV technologies address the cell efficiencies, fabrication cost, and environmental impacts in promising ways, the $1^{\text {st }}$ and $2^{\text {nd }}$ generation PV products still dominate as they constituted up to $85 \% \sim 90 \%$ of total PV market by the end of 2012 [14].

Despite the market dominance of the $1^{\text {st }}$ and $2^{\text {nd }}$ generation PV products, the past few years have also witnessed significant advances in organic photovoltaics (OPV). Compared with the traditional inorganic solar cells, organic solar cells, whose ingredients are polymers and/or small molecules, feature easy and low-cost material synthesis, low-cost fabrication, lightweight, and flexibility [15], [16]. Although the power conversion efficiency (PCE), the ratio of the maximum output electrical power to the input solar power, of organics has been much lower than those of inorganic solar cells, the advent of the bulk heterojunction (BHJ) structure [17] 
has resulted in a major performance improvement. Recently, the highest PCE of an OPV cell has surpassed 10\% [18], making the OPV a serious player among other PV technologies.

A related technology, hybrid solar cells consisting of polymers and inorganic semiconductor nanocrystals, is attractive for the next-generation photovoltaics owing to the tunable bandgap, high electron mobility, strong and broadband absorption, and the suitability for solution processing of the nanocrystals [19], [20]. However, the favorable material properties above have not been translated to practical advantages over organic solar cells. The reported PCE for hybrid BHJ solar cells has not exceeded 4\% [21], [22], leaving space for improvement.

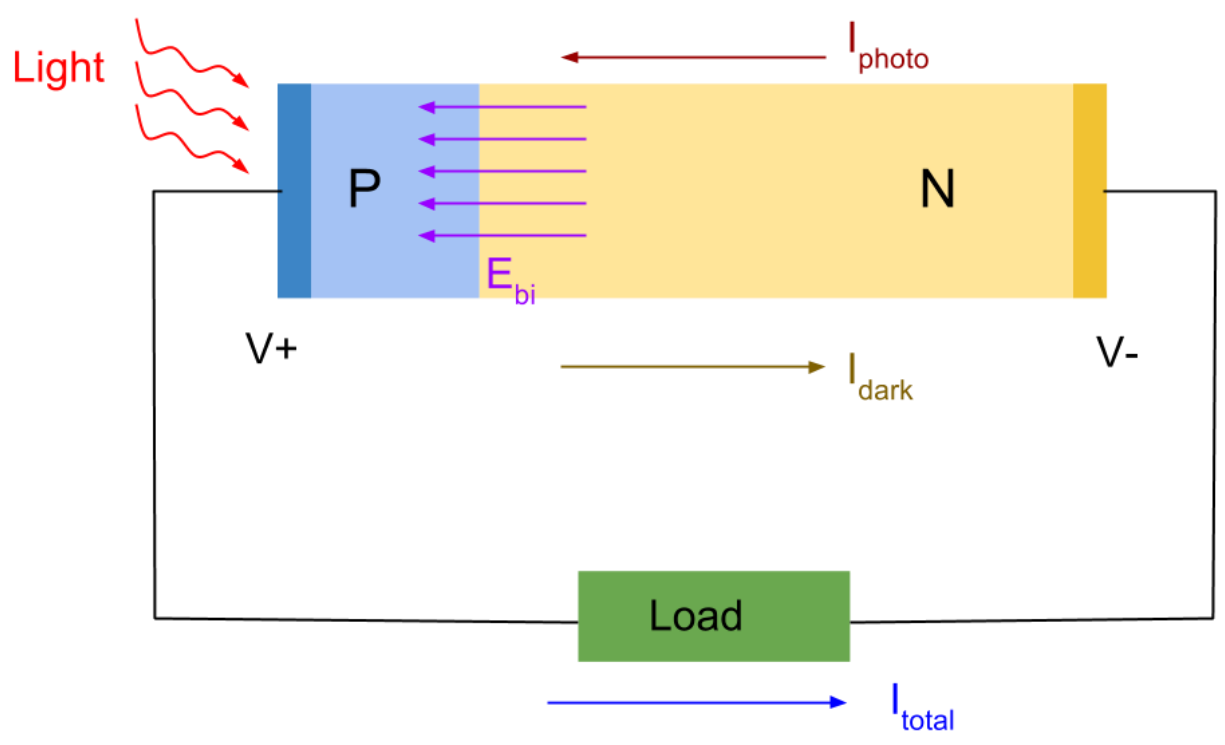

Figure 2.1: An illustration of working principles of a typical pn-junction inorganic solar cell. $E_{b i}$ is the built-in electric field in the depletion region.

Figure 2.1 illustrates the operation of a typical inorganic pn-junction solar cell (under illumination). The working principles of other types of solar cells (e.g., organic, hybrid, dyesensitized, etc.) are very similar. Electron-hole pairs are created when the incident light is absorbed. These are split and drift under the influence of the built-in electric field in the depletion region before being collected by the electrodes. Since the photocurrent flows in the direction of built-in electric field and induces a voltage across the external load, the solar cell is forward biased and the dark current flows in the opposite direction of the photocurrent. The 
amount of the total current flowing through the load depends on the physical properties of the solar cell and the load impedance.

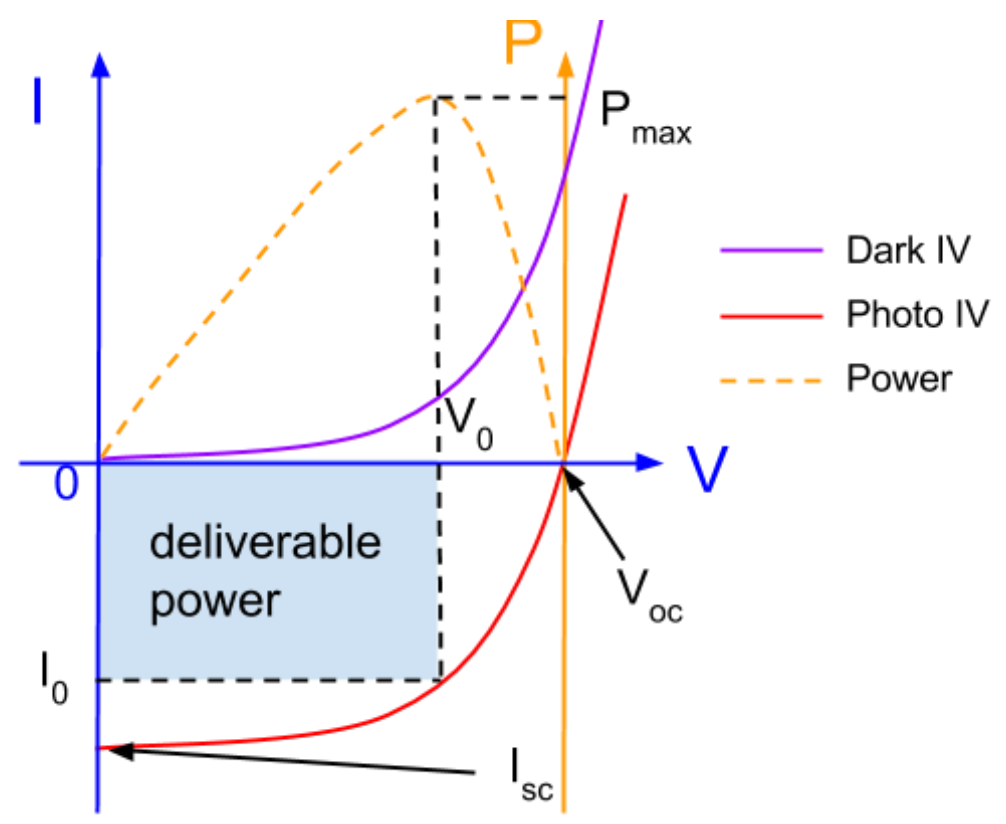

Figure 2.2: Typical current-voltage characteristics of a solar cell in dark and illuminated conditions. Important parameters, such as open-circuit voltage $V_{o c}$, short-circuit current $I_{\text {sc }}$, and maximum deliverable power $P_{\max }$, are labeled on the plot. Fill factor can be calculated from those three parameters. $V_{0}$ and $I_{0}$ are the voltage and current corresponding to the maximum power point.

Although diversified in material types and device structures, all solar cells share the same benchmark metrics and the goal is to extract maximum electrical power from the sun. Figure 2.2 shows two typical current-voltage (IV) characteristics in dark and illuminated conditions. Figure 2.2 only shows the part where the applied bias is non-negative because solar cells normally operate in the forward bias mode. When there is no illumination, the IV curve of a solar cell follows the typical Shockley diode equation [23]. When the solar cell is illuminated, the IV curve moves into the fourth quadrant. There are four important parameters that describe the performance of an illuminated solar cell as follows:

1) Open-circuit voltage $V_{o c}$ is defined as the positive voltage when there is no current flowing in the cell. It is also the maximum voltage an external circuit can sense from an illuminated solar cell. A solar cell with large energy bandgap usually has large $\mathrm{V}_{\mathrm{oc}}$. 
2) Short-circuit current $I_{s c}$ is defined as the current flowing in an illuminated cell when the applied voltage is zero. It is also the maximum current that can be extracted by an external circuit. A solar cell with large optical absorption usually results in large $\mathrm{I}_{\mathrm{sc}}$.

3) Fill factor (FF) is the ratio of the "deliverable power" to the product of open-circuit voltage and short-circuit current and is expressed as

$$
F F=\frac{P_{\max }}{V_{o c} \times I_{s c}}
$$

where $\mathrm{P}_{\max }$ is the maximum power that can be delivered to an external circuit. A solar cell with small series resistance usually yields large fill factor.

4) Power conversion efficiency (PCE) is the ratio of the "deliverable power" to the input solar power.

$$
\eta_{P C E}=\frac{P_{\max }}{P_{\text {solar }}}
$$

where $\mathrm{P}_{\text {solar }}$ is the input solar power. To standardize the measurement of PCE in laboratory conditions, the input solar power is usually calibrated to the air mass 1.5 global (AM 1.5G) solar spectrum, which is one of the standard solar spectra for the northern hemisphere as shown in Figure 2.3, with $1000 \mathrm{~W} / \mathrm{m}^{2}$ or $100 \mathrm{~mW} / \mathrm{cm}^{2}$ power density. PCE is also the ultimate metric for comparing the photovoltaic performance of different solar cells.

It is desirable to maximize all of four parameters in order to get the best power conversion efficiency. However, they are usually interrelated and trade-offs have to be made. For example, $\mathrm{V}_{\mathrm{oc}}$ can be large for a solar cell with a big energy bandgap at the cost of reduced $\mathrm{I}_{\mathrm{sc}}$, because more long-wavelength solar power is discarded, leading to reduced optical absorption. Therefore, the resulting PCE may not necessarily increase relative to a lower bandgap material with 
correspondingly lower $\mathrm{V}_{\mathrm{oc}}$. Moreover, $\mathrm{V}_{\mathrm{oc}}$ and $\mathrm{I}_{\mathrm{sc}}$ are physically linked to the material processing and the charge carrier transport which will be further discussed in Chapter 4.

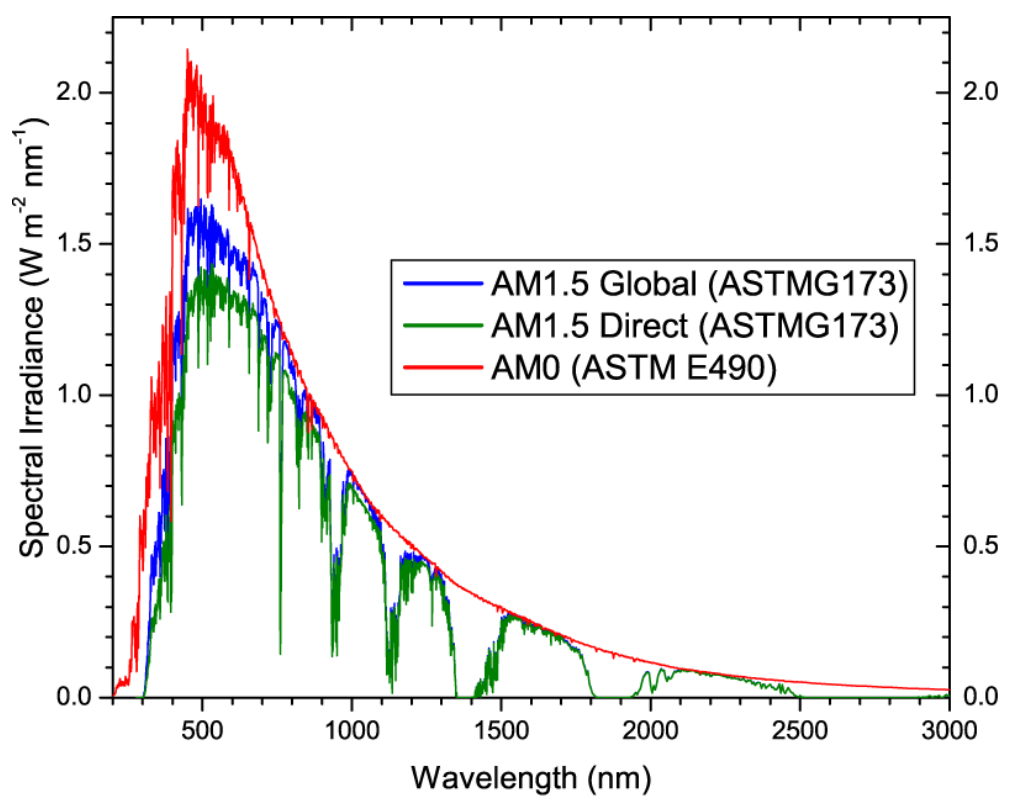

Figure 2.3: Three different standard solar spectra. The AM1.5 Global (AM1.5G) spectrum is for the terrestrial use of flat photovoltaic cells and modules with power density of $1000 \mathrm{~W} / \mathrm{m}^{2}$ or $100 \mathrm{~mW} / \mathrm{cm}^{2}$. The AM1.5 Direct (AM1.5D) is for the terrestrial use of solar concentrators. The AMO spectrum is for the photovoltaic applications in space. Usually, AM1.5G is most widely used for lab cells. This figure is from Ref [24], and the original data can be found in $\operatorname{Ref}[25]$.

\subsection{Organic Solar Cells}

Figure 2.4 shows the best power conversion efficiencies of different PV technologies. The multi-junction solar cells have achieved champion PCE owing to their ability to absorb across a broad spectrum with optimized bandgap energies. For single-junction solar cells, inorganic monocrystalline solar cells still lead both PCE and market share. In the lower right corner of the figure are the emerging PV technologies including organic and hybrid solar cells which are improving as the fabrication cost keeps decreasing and PCE increasing. 
There are two types of organic solar cells: bilayer and bulk heterojunction. In both types the exciton dissociation occurs near the interface between the n-type and p-type materials, and the excition diffusion length is no larger than a few tens of $\mathrm{nm}$. In bilayer organic solar cells (Figure 2.5 (a)), the active layer consists of two distinctive n-type and p-type organic layers. The active layer cannot be thicker than the exciton diffusion length, making the cell an inefficient light absorber. This limitation is alleviated by making the active layer an interlocked network of ntype and p-type materials as in bulk heterojunction (BHJ) organic solar cells (Figure 2.5 (b)). The excitons can diffuse to a localized p-n interface nearby without traveling through the whole active layer. Therefore the active layer of BHJ cells can be much thicker than that of bilayer cells, which explains why BHJ cells are usually more efficient than bilayer cells.

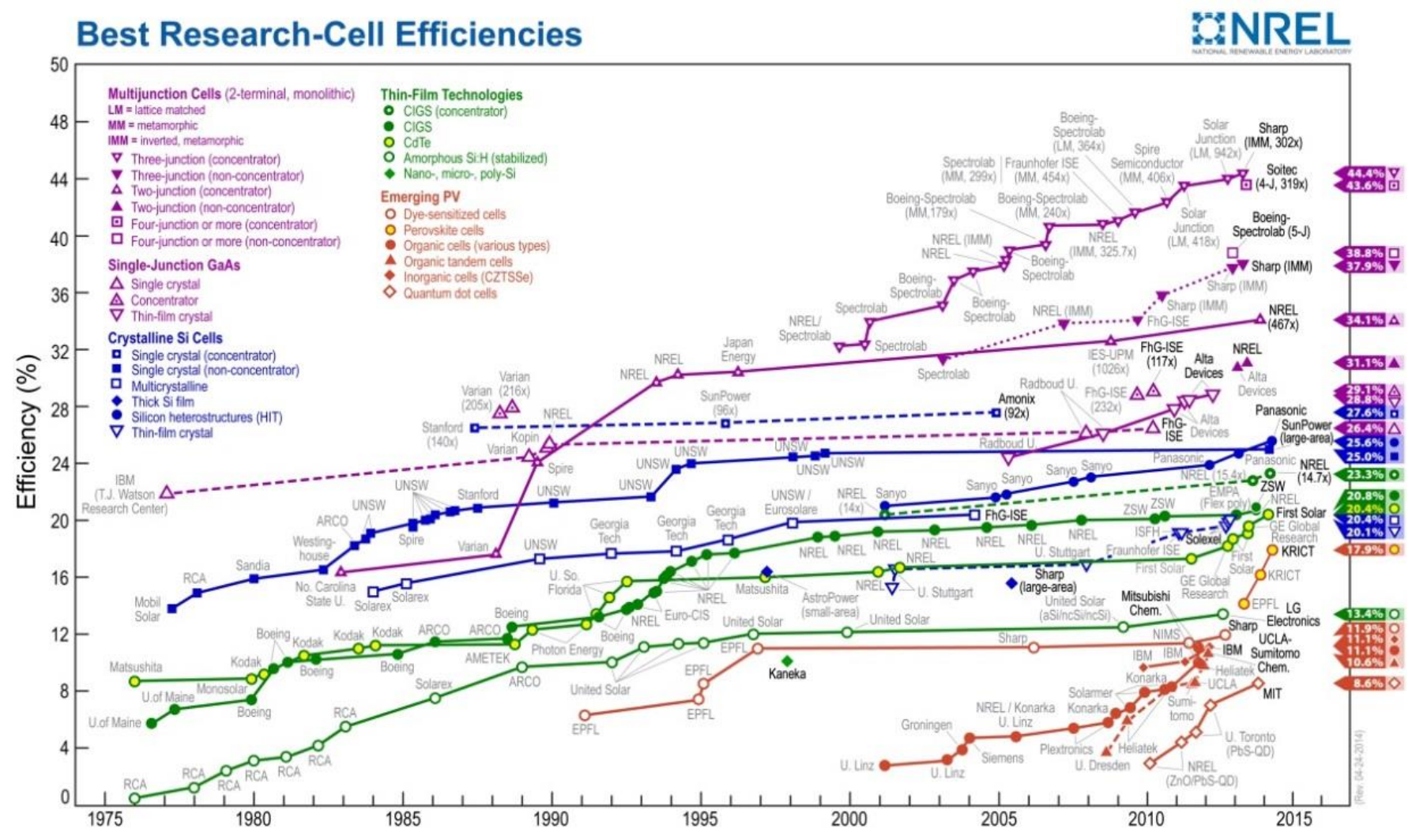

Figure 2.4: Best power conversion efficiencies of different PV cells certified by National Renewable Energy Laboratory (Source: Ref [26])

Figure 2.5(b) shows the device structures of a typical BHJ organic solar cell. For many scientific studies, a donor-type polymer, poly (3-hexylthiophene-2,5-diyl) (P3HT), and an 
acceptor-type small molecule, [6,6]-phenyl C61-butyric acid methyl ester (PCBM), are used as the reference because their properties are well known as a result of numerous studies. The energy band diagram of P3HT:PCBM organic solar cells is shown in Figure 2.6. The active layer is sandwiched between the indium tin oxide (ITO) (anode) and aluminum (cathode). The PEDOT:PSS layer is the electron-blocking layer that only permits hole transport.
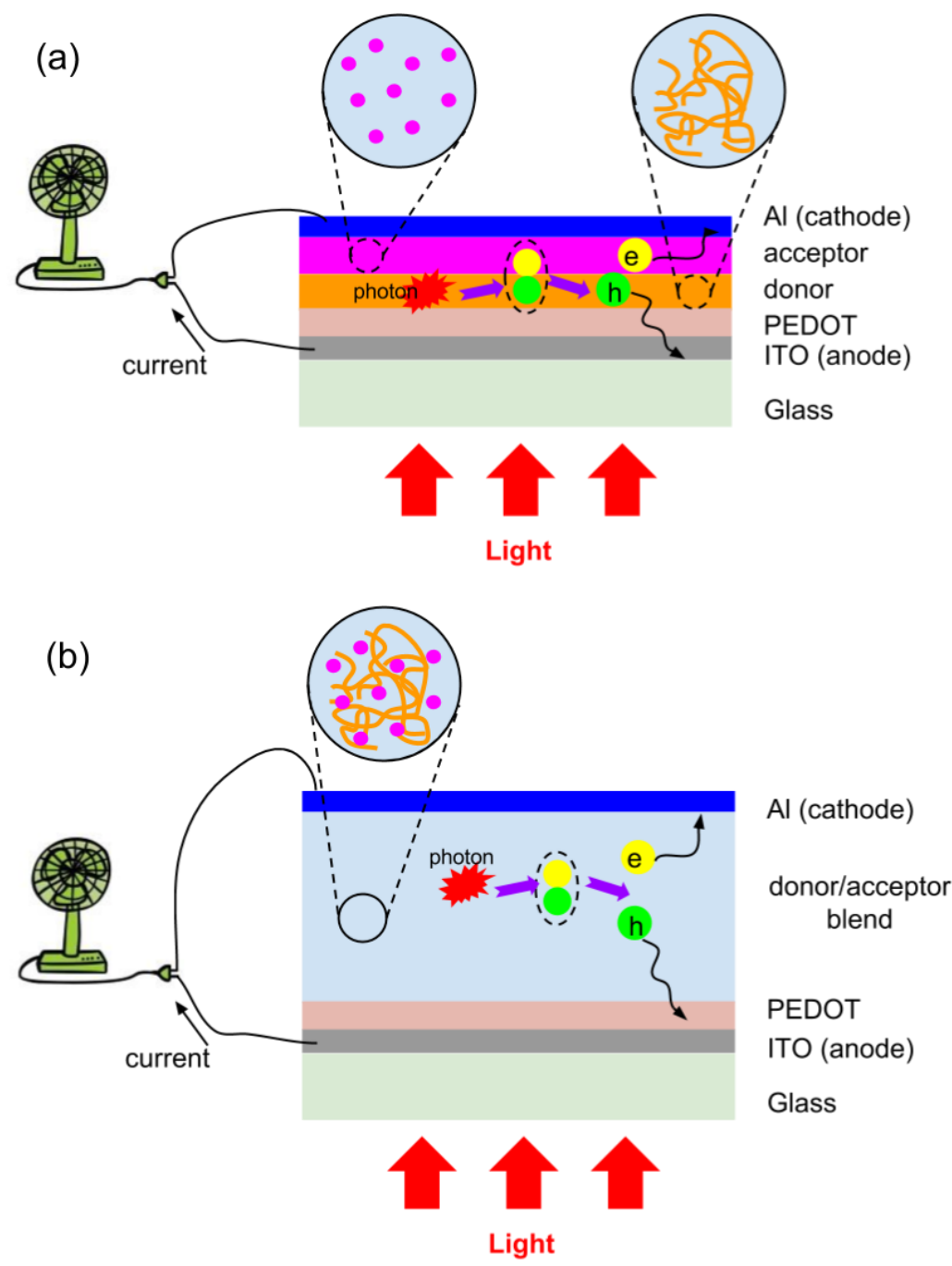

Figure 2.5: The device structures and light harvesting processes of (a) bilayer and (b) bulk heterojunction organic solar cells. The pink dots in the magnified circles stand for small molecule acceptors like PCBM, while the wiggling chains in the other magnified circles represent polymers such as $\mathrm{P} 3 \mathrm{HT}$.

However, there is a caveat about the band diagram in Figure 2.6. Because organic crystals lack long-range periodic crystalline structure, the energy band theory of inorganic crystals does not strictly apply to organics. As an analogy and for the ease of understanding the charge transport, 
the highest occupied molecular orbital (HOMO) and lowest unoccupied molecular orbital (LUMO) of organic solar cells correspond to the conduction band edge and valence band edge of inorganic solar cells. However, understanding the energetics of heterogeneous organic material systems needs more complicated molecular-level simulation. Also, there is still few work exploring the band pinning of the organic donor-acceptor heterojunction interface, which is drastically simplified in the drawing of Figure 2.6. Lots of future work is still needed to understand the most fundamental physics upon which the organic photovoltaics is built.

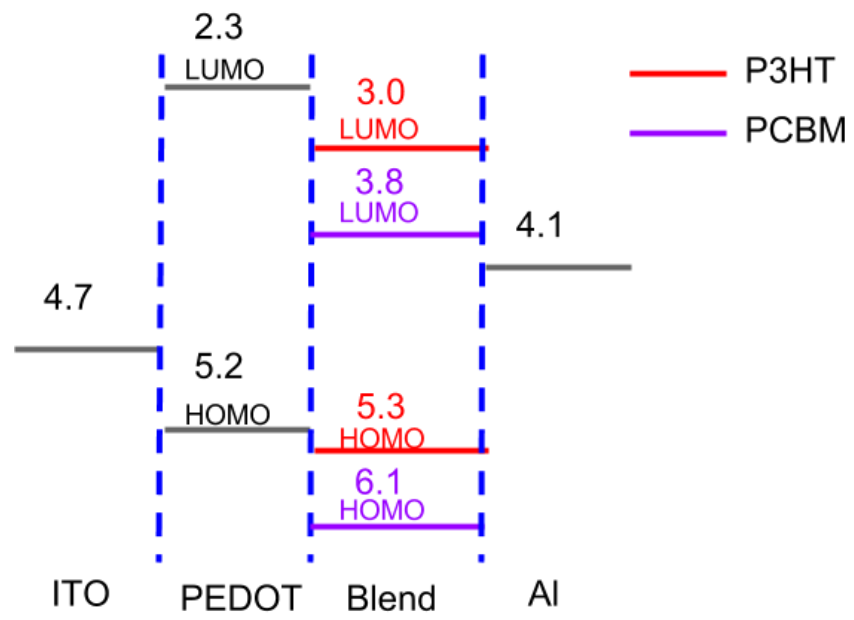

Figure 2.6: The energy band diagram of P3HT:PCBM organic solar cells.

It is also instructive to understand the energy conversion processes of BHJ organic solar cells as shown in Figure 2.7, and the subtle differences from their inorganic counterparts.

1) Absorption and exciton generation (Figure 2.7 (a)). For a typical BHJ solar cell, the light enters through the bottom glass substrate and is absorbed by the active layer. Each absorbed photon is converted to an exciton, i.e., a bound electron and hole pair. Enormous effort has been devoted to enhancing the material absorption of the solar spectrum in this step because it remains the most direct way of improving the PCE of BHJ solar cells.

2) Exciton diffusion and recombination (Figure 2.7 (b)). Unlike the fact that excitons in inorganic solar cells have low binding energies and can be easily split into separate electrons and holes by room-temperature thermal agitation, the relatively low dielectric constants of organic materials make exciton binding energies much larger than kT. Therefore, the newly- 
generated excitons in organic materials remain bound and diffuse in all directions before recombination. Only the excitons that manage to diffuse to the BHJ interfaces can proceed through the subsequent steps required to produce photocurrent. Long exciton recombination lifetime, a parameter related to the material properties of the blend, is favored in this step because it yields longer diffusion length.

3) Exciton dissociation and charge transfer (Figure 2.7 (c) and (d)). The LUMO and HOMO misalignment at the interfaces of the donor-type (e.g., P3HT) and acceptor-type (e.g. PCBM) materials induces large interfacial electrical fields. As a result, excitons will be split into separate electron and hole polarons (analogous to the free electron and hole carriers in inorganic materials) near the interface. The electron polarons will transfer to the acceptor LUMO, and the hole polarons to the donor HOMO. In this step, the choice of materials influences both the exciton binding energy and the interfacial electric field, which determine the exciton dissociation efficiency.

4) Polaron transport and collection (Figure 2.7 (e)). After the dissociation, the electron and hole polarons will travel under the influence of the built-in electric field, and finally be collected by the electrodes. Several physical processes, such as trapping and recombination, will affect the collection efficiency.

Different from inorganic crystals with long-range order where the mobile charges can freely drift in the electric field, the charges in amorphous semiconductors such as organic optoelectronic devices are localized to individual molecular sites. The charge transport in such systems occurs by "hopping". Charges localized in one site have to overcome the potential barriers either by thermal agitation or tunneling, and hop to adjacent sites. The hopping process is random in time, and the statistical hopping rate from site $i$ to site $j$ can be described by the Miller-Abraham model in Equation ( 2.3):

$$
v_{i j}=v_{0} \exp \left(-\gamma r_{i j}\right) \exp \left(-\frac{\Delta E_{i j}}{k T}\right)
$$


where $v_{0}$ is the attempt-to-escape rate, $r_{i j}$ is the distance between site $i$ and site $j, \gamma$ is a proportionality factor related to how easily the charge can hop from site $i$ to site $j, \Delta E_{i j}$ is the energy difference between states at site $\mathrm{i}$ and site $\mathrm{j}, \mathrm{k}$ is the Boltzmann's constant and $\mathrm{T}$ is the temperature.

The charge hopping mechanism renders charge transport in organic semiconductors less mobile than inorganic crystals, and it is the major reason why the charge carrier mobility in organic solar cells is much lower than in inorganic crystalline solar cells.

(a)

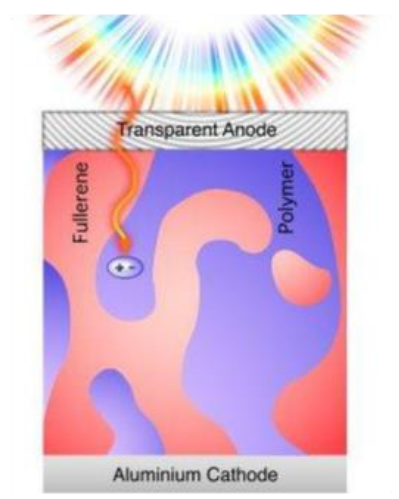

(b)

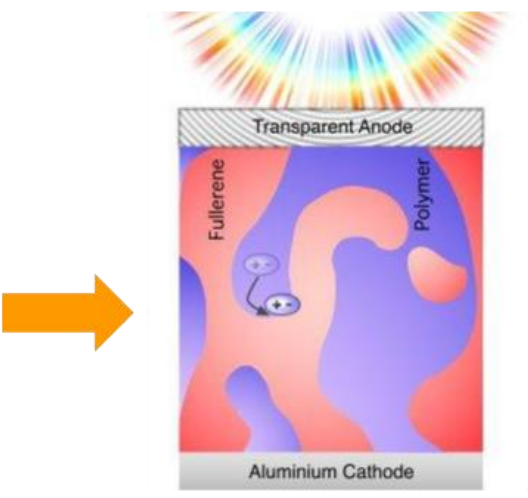

(c)

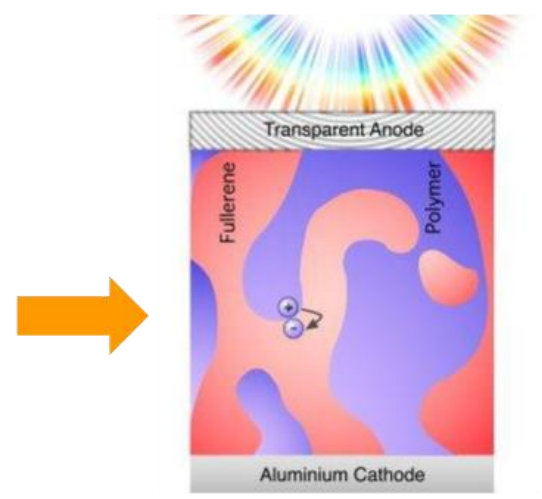

(e)

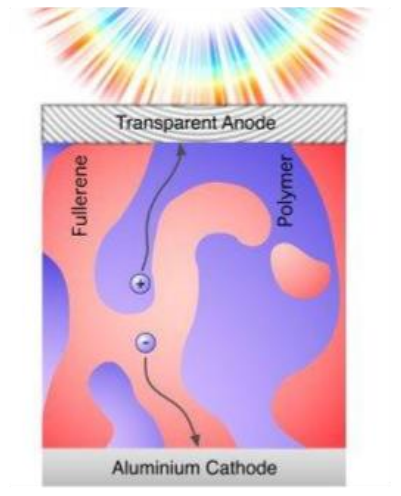

(d)

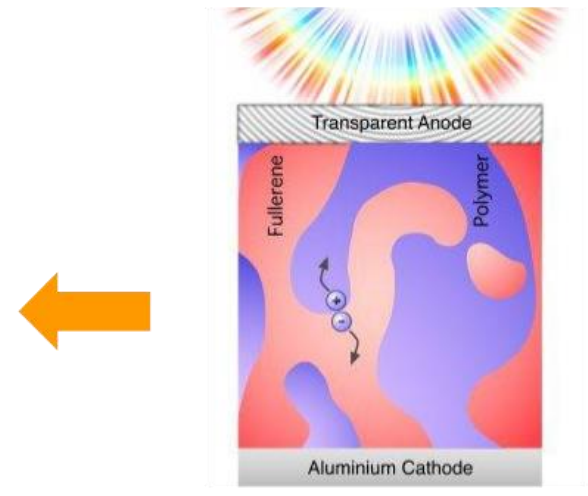

Figure 2.7: The light harvesting processes of bulk heterojunction organic solar cells. 
(2)

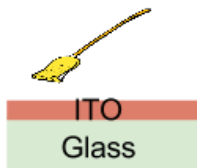

(3)

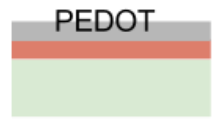

(8)

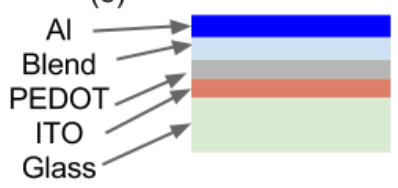

(1)

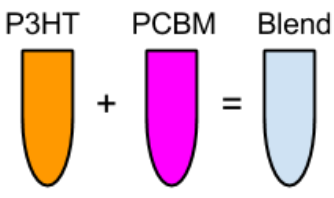

(4)

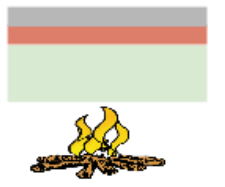

(7)

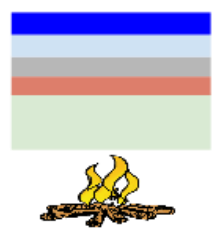

(5)

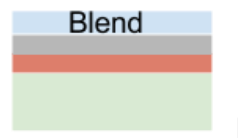

(6)

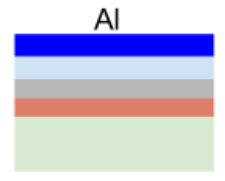

Figure 2.8: Step-by-step fabrication process of typical P3HT:PCBM bulk heterojunction organic solar cells

Compared with inorganic material growth and fabrication which may require high temperature, gas pressure control, poisonous exhaust handling, and sophisticated patterning, the fabrication of organic solar cells is relatively easy. The multilayer stack is realized by solution based spin-casting techniques, and the crystallization by thermal treatment. Figure 2.8 illustrates the step-by-step fabrication of typical P3HT:PCBM BHJ organic solar cells, which is described as follows:

1) P3HT (from Rieke Metals) and PCBM (from American Dye Source) are dissolved and stirred separately in chlorobenzene for 12 hours before being mixed and stirred for another 12 hours at room temperature. The density is $2 \mathrm{wt} . \%$ in total, composed of a ratio of 11:9 of P3HT to PCBM.

2) Indium tin oxide (ITO)-coated glass substrates are ultrasonically cleaned with acetone, isopropyl alcohol, and de-ionized water and subsequently blow dried by compressed air.

3) Highly conducting aqueous PEDOT:PSS (Clevious PH 500) solution is spin-coated onto the ITO substrates with a spin rate of $4000 \mathrm{rpm}$.

4) The substrates are baked for 15 minutes at $120{ }^{\circ} \mathrm{C}$ in vacuum.

5) Another spin-coating of P3HT:PCBM blend with a spin rate of $900 \mathrm{rpm}$ is carried out.

6) After that, the 100-nm Al top electrode layer is thermally evaporated onto the blend. 
7) The sample is annealed at certain temperature (usually between $120^{\circ} \mathrm{C}$ and $140^{\circ} \mathrm{C}$ ) for 30 minutes in vacuum. The annealing process helps the crystallization of the P3HT phase and the aggregation of the PCBM phase.

8) Finished P3HT:PCBM cells as pictured in Figure 2.9 are ready for further tests.

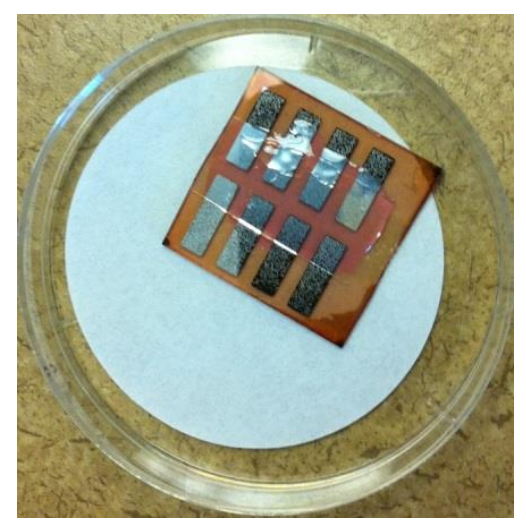

Figure 2.9: P3HT:PCBM bulk heterojunction solar cells fabricated in our lab. There are eight devices on the substrate.

\subsection{Photodiodes}

Photodiodes are a type of widely used photodetectors that convert optical signals to electrical signals. Compared with photomultiplier tubes (PMT), photodiodes are compact and do not require extremely high voltage (usually hundreds of volts) to operate. Compared with chargecoupled devices (CCD), photodiodes respond much faster. Devices based on superconducting materials are also very promising photodetectors with very high responsivity and speed [27]. However, they usually require low temperatures to bring the material to superconducting mode. In many applications such as fiber optic communications, biological agent detection and single photon counting, a photodiode is the solution featuring high sensitivity, high speed and small form factor.

As shown in Figure 2.10, the basic working principle of a pn-junction photodiode is very similar to a solar cell (Figure 2.1). Photodiodes are frequently used for signal-conditioning 
beyond mere power conversion. There are three important metrics in terms of the performance of a photodiode:

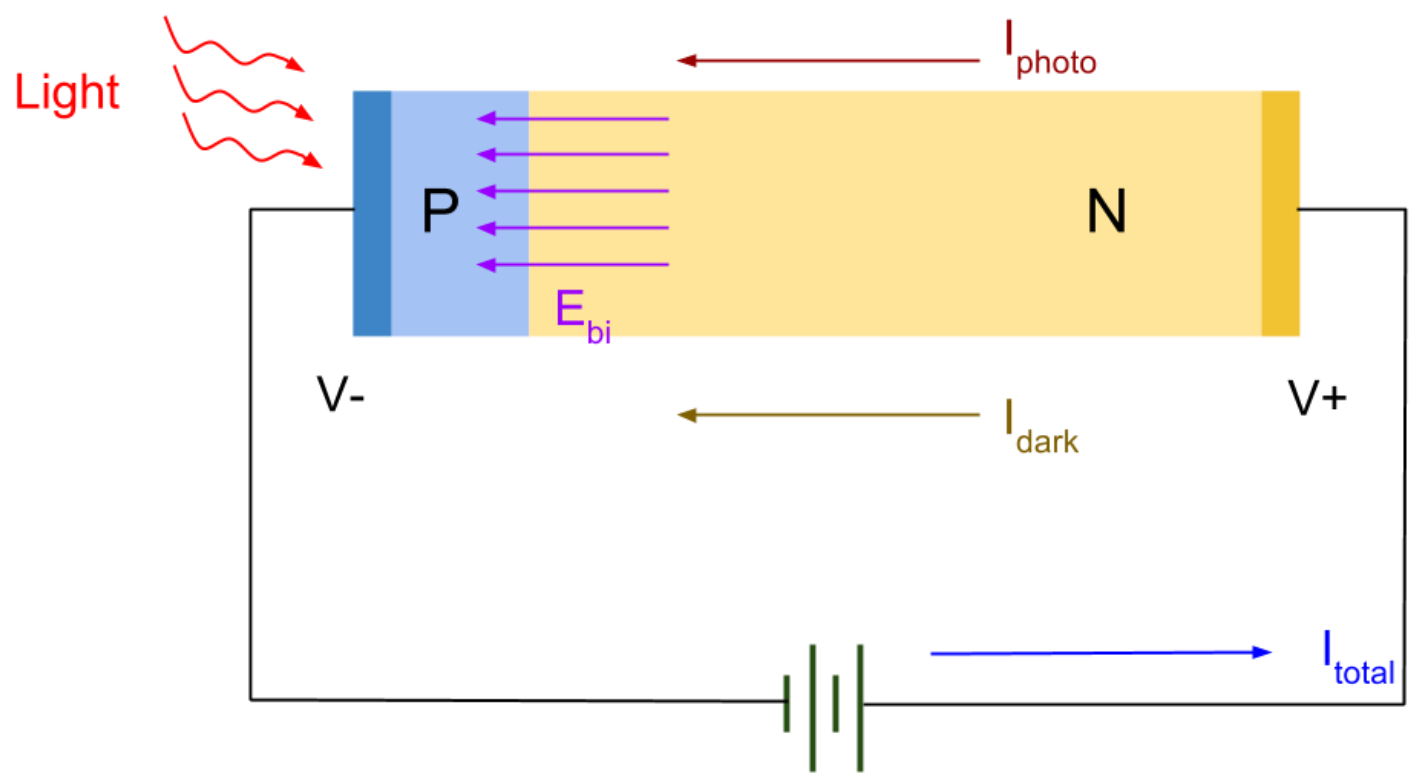

Figure 2.10: An illustration of a p-n junction photodiode in working condition.

1) Responsivity is defined as the ratio of output photocurrent to the incident optical power; The responsivity can be expressed as

$$
R=\frac{I_{p h}}{P_{\text {opt }}}=\eta_{E Q E} \frac{\lambda[\mu m]}{1.24}
$$

where $I_{p h}$ is the output photocurrent, $P_{\text {opt }}$ is the incident optical power, $\eta_{E Q E}$ is the external quantum efficiency, and $\lambda$ is the wavelength of the incident optical signal with the unit of $\mu \mathrm{m}$. The external quantum efficiency is further related to the material absorption and surface reflection, which can be enhanced by increasing the thickness of the absorbing region and incorporating anti-reflection coatings, at the cost of device speed and processing expense.

2) Bandwidth. The $(3 \mathrm{~dB})$ bandwidth of a photodiode is the highest frequency at which the output RF power decreases to half its value at low frequency. The larger the bandwidth, the higher the speed of the device. There are two mechanisms that limit the bandwidth of the device. The first is the RC limit inherent to the first-order low-pass RC system formed 
by the junction capacitance of the device, $\mathrm{C}_{\mathrm{j}}$, and the load resistance, $\mathrm{R}_{\mathrm{L}}$. The $3 \mathrm{~dB}$ bandwidth relation to the $\mathrm{RC}$ limit is

$$
f_{3 d B, R C}=\frac{1}{2 \pi R_{L} C_{j}} .
$$

The second limiting factor is the finite transit time required to collect carriers. This is usually the time to reach the contact region. The $3 \mathrm{~dB}$ bandwidth associated with the transit time limit is estimated as

$$
f_{3 d B, t r}=\frac{3.5 v}{2 \pi d}
$$

where $\mathrm{v}$ is the average carrier velocity, and $\mathrm{d}$ is the thickness of the device. The measured $3 \mathrm{~dB}$ bandwidth of a photodiode is given by [28]

$$
\frac{1}{f_{3 d B}^{2}}=\frac{1}{f_{3 d B, R C}^{2}}+\frac{1}{f_{3 d B, t r}^{2}}
$$

Shrinking the active area of the device will increase $f_{3 \mathrm{~dB}, \mathrm{RC}}$ without affecting the carrier transit time. However, manipulating the thickness of the device will incur a trade-off: decreasing the thickness of the device will decrease $f_{3 d B, R C}$ but increase $f_{3 d B, t r}$.

3) Linearity. The output of a photodiode is a linear function of the input only for smallsignals. Non-linearity occurs when the photodiode is driven to saturation by large inputs. Linearity can be understood as the range where the output follows the input without noticeable distortion. The figures of merit for the linearity are the $1 \mathrm{~dB}$ compression point and the $3^{\text {rd }}$-order intercept (IP3) point. The dashed green line in Figure 2.11 is the ideal linear response of a photodiode extrapolated from the low signal level, while the red line is the measured response. The $1 \mathrm{~dB}$ compression point is defined as the output RF power where the measured response is $1 \mathrm{~dB}$ lower than the ideal linear response, which is $\mathrm{P}_{1 \mathrm{~dB}}$ in Figure 2.11. As the input further increases, higher order harmonics appear, and the IP3 
point is the output power where the ideal linear response and $3^{\text {rd }}$-order response intercept. Since the lower bound of the operating power for photodiodes is set by the noise floor, high $1 \mathrm{~dB}$ compression point and IP3 point are always desired for high dynamic range.

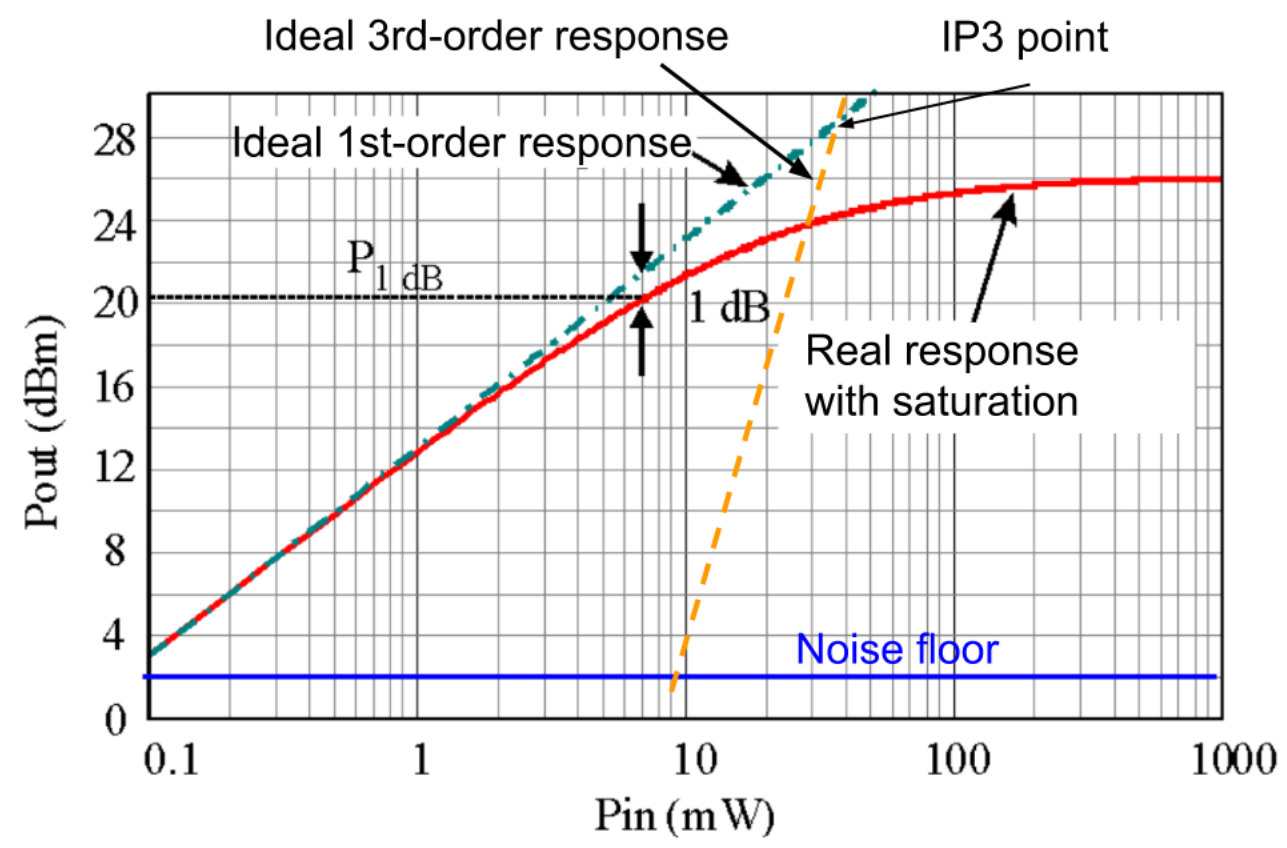

Figure 2.11: An illustration of the figures of merit for photodiode linearity. The image is based on the class notes of ECE6261 Microwave Engineering II taught by Bobby Weikle at the University of Virginia.

Since the targeted market for photodiodes is very different from that of solar cells, the design goals of photodiodes and solar cells are different in many aspects. Some of those are:

- Speed is an important metric for photodiodes because they are widely used in optical communication systems, but is rarely considered for solar cells. The active area of photodiodes is usually a trade-off between the sensitivity and speed, while solar cells are usually designed for large-surface application to harvest as much sunlight as possible.

- Photodiodes usually work under reverse bias condition provided by an external voltage source, while solar cells are always forward biased as discussed previously in this chapter. Reverse bias can increase the electric field in the depletion region of a photodiode and increase its speed.

- Noise performance is very important for photodiodes, which is rarely considered for 
solar cells.

- The output photocurrent and its properties are of great interest for a photodiode, while for solar cells, the extracted power is more important than a single parameter such as photocurrent.

At the same time, photodiodes and solar cells share some of the same metrics such as

- Energy conversion efficiency. Both try to extract the maximum electrical signal from an input optical signal. For photodiodes, large responsivity is desired. For solar cells, higher power conversion efficiency is the ultimate goal.

- High current/power handling. For some applications it is important for the device to operate without degradation when being stressed to extremes. For example, high-power high-speed photodiodes such as modified uni-traveling carrier (MUTC) photodiodes are frequently driven to saturation. In this case, non-linearity plays a non-negligible role and thermal dissipation becomes a problem. For concentrated photovoltaic (CPV) modules, the input optical power after focusing the solar radiation can be many times large than other usual PV applications. And the resulting photocurrent is also significantly larger.

A complete survey of photodiodes and their diverse applications is beyond the scope of this chapter. For the interest of this dissertation, we are interested in the high-power and high-speed modified uni-traveling carrier (MUTC) photodiodes that are very useful for applications such as analog optical links [29]-[31] and low phase noise microwave signal generation [7]. The noise performance of MUTC photodiodes will be discussed in Chapter 5.

Figure 2.12 shows the band diagram and device structure of an MUTC1 photodiode [32]. Although the device structure looks much more complicated than a typical solar cell, it is essentially a diode with p-type InGaAs layers as the absorber and n-type InP as the electron collector. As most absorption occurs in the p-region, the holes can be easily collected while the electrons have to diffuse to the depletion region to be swept to the n-region. Therefore, the charge transport of the electrons is a design focus for increasing the device speed and optical power handling. 


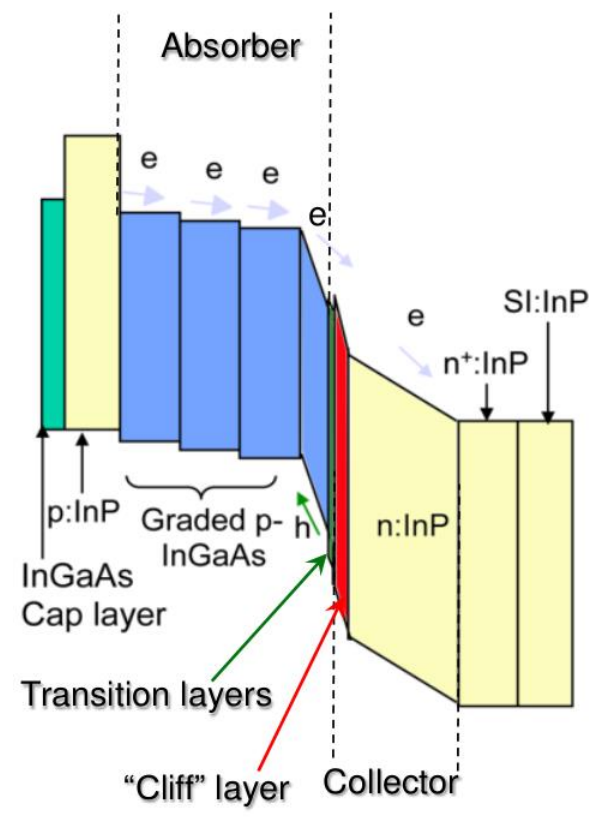

(a)

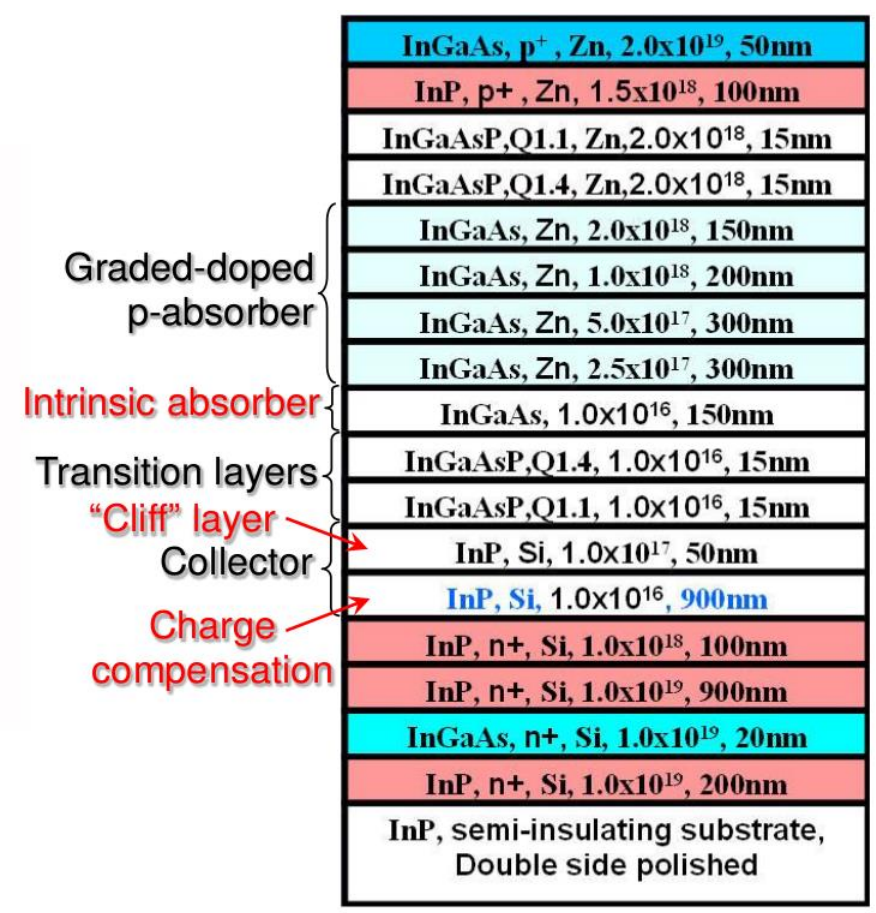

(b)

Figure 2.12: The (a) band diagram and (b) device structure of a MUTC1 photodiode. The image is from Ref[33].

MUTC photodiodes are designed for high-speed and high-power operations. Several design modifications have been implemented to improve the charge transport of the electrons. For example, a highly-doped n-type InP layer, also called the "cliff layer", is inserted to increase the electric field in the depletion region so that the electrons can be collected more efficiently. Also, the doping in the undepleted p-type InGaAs absorber is graded, which forms an electric field that assists diffusion of photogenerated electrons toward the depletion region. When the photocurrent is high, there is another "self-induced" electric field that originates from the ohmic voltage drop across the undepleted absorber. Its directions is from the n-region to the p-region. This "selfinduced" field can further facilitate the electron transport [33]. 


\section{Chapter 3 : Transient Photocurrent Method}

\subsection{Introduction}

For optoelectronic devices, the charge carrier transport after the optical absorption plays a crucial role in the final device performance. Good charge carrier transport usually implies

- Few carriers are lost due to recombination, which, in turn, implies high quantum efficiency is desirable for essentially all photodiodes and solar cells.

- Charge carriers can be extracted quickly. Quick charge extraction not only increases the speed of the photodiodes, but can also mitigate the space-charge effect when the photodiodes operate in the large-signal condition. For solar cells, quick charge extraction counteracts the chance of recombination and thus improves the charge collection efficiency.

- Carrier trapping is not significant. Trapping is a physical process in which mobile carriers are captured by "traps" formed by material defects or impurities before recombining with another carrier or being released to participate in the charge carrier transport. For some types of optoelectronic devices such as Geiger-mode avalanche photodiodes (APDs), carrier trapping can be especially detrimental because the released carriers that were trapped in previous operating cycles, although small in number, can trigger avalanches in subsequent detection periods. In amorphous material systems such as organic solar cells, the carrier trapping is ubiquitous, and is closely related to important photovoltaic metrics such as open-circuit voltage, short-circuit current, and power conversion efficiencies.

Charge carrier mobility and lifetime are two important parameters related to the physical properties of optoelectronic devices. Measuring those two parameters can reveal the essential information directly related to the final device performance. There have been various ways to measure these parameters, some of which are briefly described as follows:

- Time-of-flight (ToF) method [34], [35] is a very popular transient technique to measure the mobility of semiconductor materials. Charges are injected into a semiconductor material either electrically or optically, and the transit time of the charges in the material 
is measured, from which the mobility is calculated. Originally developed for the inorganic semiconductors, this approach may need revision when being applied to organic devices. Details will be discussed later this chapter.

- Space-charge limited current (SCLC) method [36], [37]. When a semiconductor material or device is stressed with high current injection, the space-charge effect occurs as the current-voltage characteristics deviate from the normal Ohm's Law (for a homogenous semiconductor slab) or Shockley's Equation (for a diode). The mobility can be extracted by fitting the steady-state current-voltage data with the corresponding physical model. It is known that trapping in semiconductors may alter the details of the SCLC model [38], [39]. Therefore, there are always caveats when applying the SCLC method to measure the charge carrier mobility in amorphous material systems.

- Carrier extraction by the linearly increased voltage (CELIV) method [40]. As its name suggests, for this method, a linearly increased voltage is applied on the device after charge injection, and the current in the external circuit is measured. Mobility is extracted by fitting the physical model governed by a Ricatti-type ordinary differential equation.

- (Organic) field-effect transistor (FET) method [41]. The mobility can be obtained by fitting the steady-state current-voltage characteristics of a FET-type device under different source-to-drain and gate-to-source voltages. However, a FET has to be fabricated using material to be measured, which may not be as straightforward as other device structures such as diodes. For organic crystals, it is well known that the carrier transport is highly anisotropic owing to the alignment of polymer chains [42]. Given that the carriers travel parallel to the channel and the layer interfaces in a FET, the mobility measured by the FET method may not reflect the mobility in the direction perpendicular to the layer interfaces, which is usually the direction of current flow in organic solar cells and organic light emitting diodes.

- Impedance spectroscopy method [43], [44]. For this characterization method, the impedance of the material systems as a function of frequency is measured. Various physical parameters, including the mobility and lifetime, can be extracted by modeling the whole system as an impedance circuit network.

- The forward-to-zero-bias transient current method [23] can measure the minority carrier lifetime of a semiconductor diode by observing the current decay under zero bias after a 
certain period of forward bias. Originally developed for inorganic materials, this method can be extended to organics as well [45].

- Forward bias transient photoconductivity method [46]. This is another way of measuring the charge carrier recombination lifetime in organic diodes by measuring the transient decay lifetimes under a series of different forward biases. The lifetimes are expected to saturate as the internal electrical field decreases (forward bias increases), and the saturation value is the recombination lifetime. Details of this method will be discussed later.

In this chapter, we study the charge carrier transport in organic material systems. We measure the mobility and recombination lifetime of two types of bulk heterojunction organic solar cells by analyzing the falling edge of their transient photocurrents. Qualitative information related to the material properties can also be obtained by studying the shape of the transient photocurrents.

\subsection{Transient Photoconductivity for Organic Solar Cells}

Figure 3.1(a) shows the experimental concept of the transient photoconductivity method. Traditionally, a slab of semiconductor material is sandwiched between to non-injection contacts, and a voltage bias is applied without injecting carriers into the semiconductor. A pulsed light is shone on one side of the slab (e.g., through the transparent or semi-transparent contact). This creates a sheet of photogenerated carriers. The thickness of the slab should be much larger than the optical absorption length so that the dimension of the photogenerated carrier sheet is negligible compared to the width of the material. After that, while one type of carriers is quickly collected by one contact, the other type of carrier will drift under the influence of the electrical field before being collected by the contact at the other end. The photocurrent can be written as

$$
J_{p h}(t)=q n(t) \mu E
$$


where $\mathrm{q}$ is the unit electric charge, $\mathrm{n}(\mathrm{t})$ is the carrier density of electrons or holes inside the semiconductor, $\mu$ is the mobility, and $\mathrm{E}$ is the electric field.
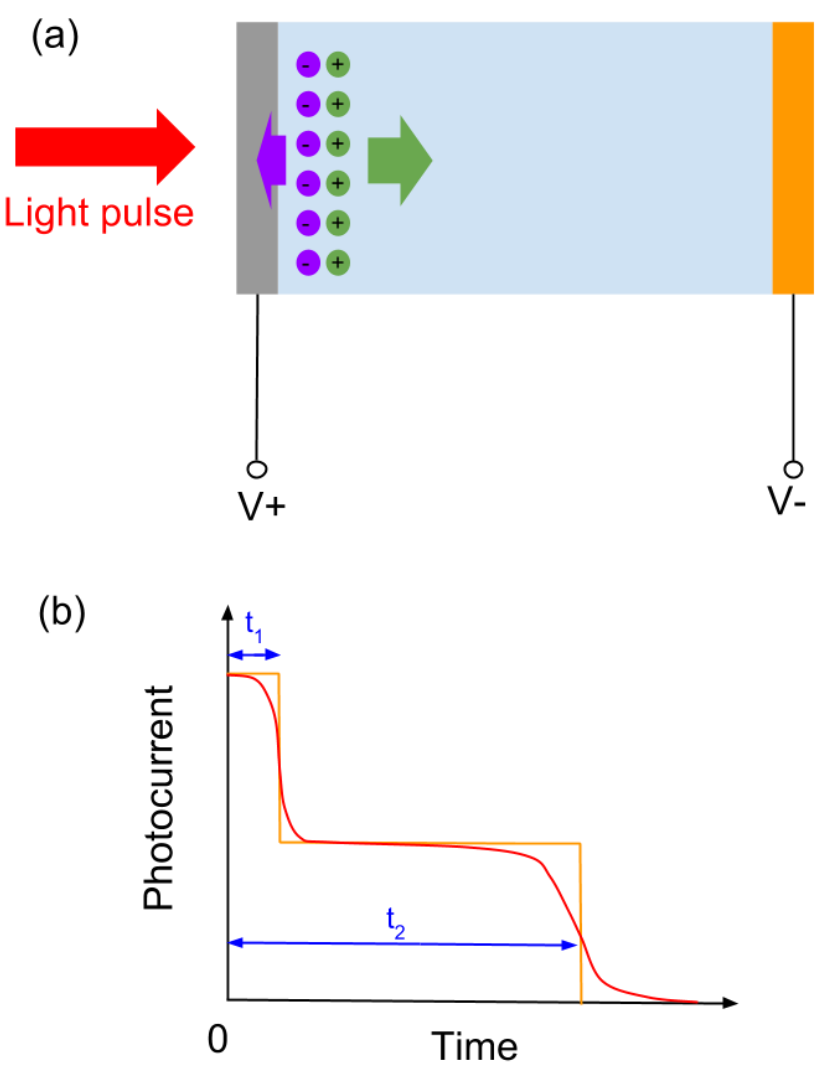

Figure 3.1: (a) The experimental concept of transient photoconductivity and (b) the typical transient photocurrent in an inorganic semiconductor where both electrons and holes participate in the charge transport. The orange solid line is the transient photocurrent without diffusion, while the red solid line is the transient photocurrent with the diffusion.

Typical transient photocurrents are plotted in Figure 3.1(b). If we ignore the diffusion of carriers, there should be two distinctive falling edges in the transient photocurrents: $t_{1}$ for fast carriers and $t_{2}$ for slow carriers. The knee after the first falling edge in the photocurrent curve appears when the decay of the faster carriers finishes but the decay of the slower carriers is still ongoing. The corresponding carrier mobilities can be calculated by

$$
\mu=\frac{L}{t_{t r} E}=\frac{L^{2}}{t_{t r} V_{a}}
$$


where $\mathrm{L}$ is the thickness of the semiconductor slab, $t_{t r}$ is the transit time (either $t_{1}$ or $t_{2}$ in Figure 3.1(b)), and $\mathrm{V}_{\mathrm{a}}$ is the applied bias. Equation ( 3.2) also assumes that the electric field is uniform inside the semiconductor slab, and the number of injected photogenerated carriers is not sufficient to induce the space-charge effect, which distorts the steady-state electric field.

The transient photoconductivity method can also be applied to a thin-film organic solar cell. However, the details differ from the traditional method in several ways:

- The assumption of "delta-function" photogenerated carriers in space may not be valid. The thickness of the BHJ organic device, usually around $100 \mathrm{~nm}$, is not optically thin enough, and the pulsed light will generate electron-hole pairs throughout in the film. If we approximate the photogeneration profile in the organic thin film to be uniform, the effective transit length of the carriers before the being collected by the electrodes is half the device length. Therefore, the mobility equation becomes [46]

$$
\mu=\frac{L}{2 t_{t r} E}=\frac{L^{2}}{2 t_{t r} V_{a}}
$$

- For organic solar cells, owing to pervasive trapping, the charge transport is very "dispersive". In dispersive transport, the mobility can be modeled as a time-dependent parameter, and there are almost no distinctive falling edges. Dispersive transport also has other interesting properties that are not usually seen in inorganic crystalline systems [46][48].

Note that for the application of transient photoconductivity, the transit time is determined by linear fitting of the slopes in a log-log plot of the initial photocurrent decay [46], [49].

\subsection{Transient Photocurrents of Polymer-Fullerene Bulk Heterojunction Organic Solar Cells}


Our collaborators, Burkhart et al. in Dr. Barry Thompson's group at the University of Southern California (USC), have developed a family of "semi-random" alkythiophene-based copolymers with a much broader absorption spectrum compared with the classical poly(3hexylthiophene) (P3HT) [50]. However, several of these broadband absorbers fail to achieve the power conversion efficiency of P3HT:6,6-phenyl-C61-butyric acid methyl ester (PCBM) bulk heterojunction (BHJ) solar cells. The DC performance of those two types of solar cells, based on P3HT and poly(3-hexylthiophene) thiophene thienopyrazine (P3HTT-TP), is shown in Table 3.1. It is obvious that P3HT:PCBM cells are much better than P3HTT-TP:PCBM cells. In fact, the efficiency of P3HTT-TP:PCBM solar cells is approximately 5 6 times lower than P3HT:PCBM BHJ solar cells. It is important to understand the causes of such a difference.

Table 3.1: The DC photovoltaic characteristics of P3HT:PCBM and P3HTT-TP:PCBM bulk heterojunction organic solar cells.

\begin{tabular}{|l|l|l|l|l|}
\hline & $\mathrm{V}_{\text {oc }}(\mathrm{V})$ & $\begin{array}{l}\mathrm{J}_{\text {sc }} \\
\left(\mathrm{mA} / \mathrm{cm}^{2}\right)\end{array}$ & FF & PCE (\%) \\
\hline P3HT:PCBM & 0.58 & 8.1 & 0.58 & 2.7 \\
\hline $\begin{array}{l}\text { P3HTT- } \\
\text { TP:PCBM }\end{array}$ & 0.37 & 1.9 & 0.33 & 0.24 \\
\hline
\end{tabular}

The synthesis of the "semi-random" alkythiophene-based copolymers is documented in Ref[50] and was carried out in Dr. Barry Thompson's group at USC. Figure 3.2(a) shows the layer structure of the fabricated polymer-fullerene BHJ devices. Figure 3.2(b) illustrates the transient measurement circuit. The pulsed light source is an Opnext laser diode (HL6363MG-A, $640 \mathrm{~nm}$ ) driven by an Agilent 81110A pulse generator with $100 \mu \mathrm{s} \sim 150 \mu$ s pulse width and 50 $\mathrm{Hz}$ repetition rate. The low duty cycle ensures that, after each pulsed excitation, the device relaxes to its steady-state before the next pulse. The light pulse rise and fall times ( 20 ns) are short relative to the response of the device. In order to be consistent with the experimental condition of the DC characterization, the optical pulse intensity is adjusted to match the $\mathrm{J}_{\mathrm{sc}}$ under 1 sun condition for each cell. The device under test is connected in series with an Agilent Infiniium DSO81004B digital oscilloscope. The input impedance of the oscilloscope is $50 \Omega$ and the device is biased using a HP 3314A function generator working in DC mode. 
Figure 3.3 (a) and Figure 3.3 (b) show the measured transient photocurrents of P3HT:PCBM and P3HTT-TP:PCBM, respectively. We observe that there are transient peaks at the rising edges in Figure 3.3(b) for all bias voltages, but none in Figure 3.3 (a). Similar phenomenon was reported by McNeill et al. in all-polymer solar cells [51]. This behavior can be explained by the model conceived by Goodman and Rose [36]. The transient peaks generally appear in a disordered material system in which electrons and holes have very different mobility-lifetime products $(\mu \tau)$. When the light is first turned on, electrons (with larger $\mu \tau$ ) reach the contact more quickly than holes (with smaller $\mu \tau$ ). This leads to an initial accumulation of holes inside the solar cell. As the space charge builds up, the electric field near both contacts experiences a selfadjusting process that extracts holes faster and electrons more slowly. The change of carrier recombination profile in time is responsible for the self-adjustment of the electric field. If the material system has very high $\mu \tau$ difference, the decreasing electron current cannot be fully compensated by the increasing hole current. On reaching steady state, the total current is smaller than the initial value, resulting in a transient peak.

It is known that electron mobility is at least an order of magnitude larger than hole mobility in organic BHJ solar cells, and large hole mobility helps improve power conversion efficiency of a solar cell [52]. The difference in the appearance of transient peaks indicates that the $\mu \tau$ difference is larger in P3HTT-TP:PCBM than in P3HT:PCBM organic solar cells. Therefore, the P3HT:PCBM cell is inherently superior to the P3HTT-TP:PCBM cell in achieving higher power conversion efficiency with more balanced charge transport. 
(a)

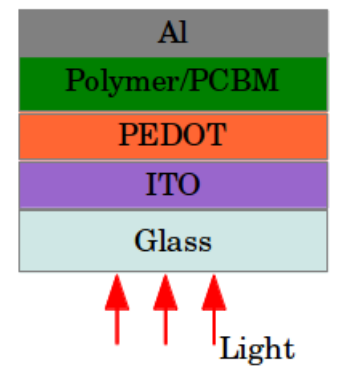

(b)

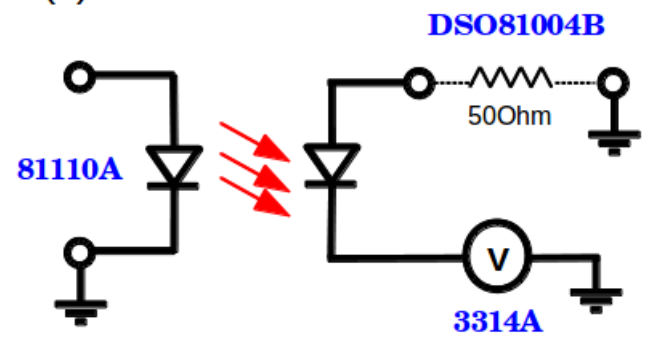

Figure 3.2: (a) Device structure of P3HT:PCBM and P3HTT-TP:PCBM bulk heterojunction organic solar cells. (b) The measurement circuit for transient photocurrents.

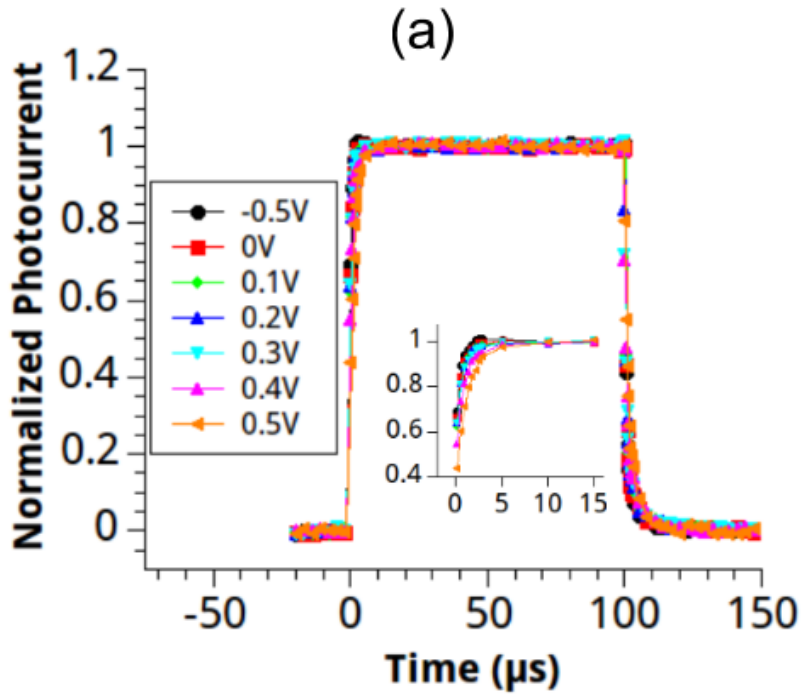

(b)

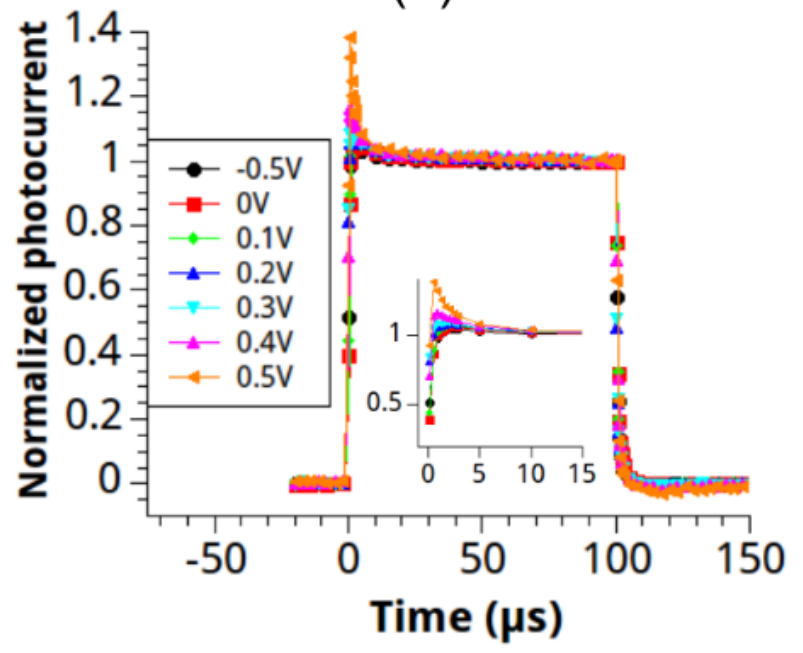

Figure 3.3: The normalized transient photocurrents of (a)P3HT:PCBM and (b)P3HTTTP:PCBM ВHJ organic solar cells excited by $640 \mathrm{~nm}$ light pulses. $J$ is the photocurrent density. Both devices are measured under 1 sun equivalence.

A further investigation of the falling edges of the transient photocurrent yields a more quantitative understanding of the difference described above. The voltage dependent transient photocurrent decay reflects the competition between charge carrier sweep-out and recombination [46]. When a solar cell is under reverse bias, the internal electric field is large enough to sweep out photogenerated carriers before significant carrier recombination occurs. The charge carrier sweep-out time can be measured using the time-of-flight method. However, when the internal voltage decreases as the applied bias changes from the reverse to forward, the carrier sweep-out 
slows down. Carrier recombination eventually dominates when the applied bias nears the built-in voltage, which is very close to the open-circuit voltage. At that point the photocurrent decay primarily reflects carrier recombination prior to collection by the electrodes. Therefore, by measuring the transient photocurrent decay time as a function of applied bias, we can extract the carrier recombination lifetime as the decay time reaches its maximum near the built-in voltage.

(a)
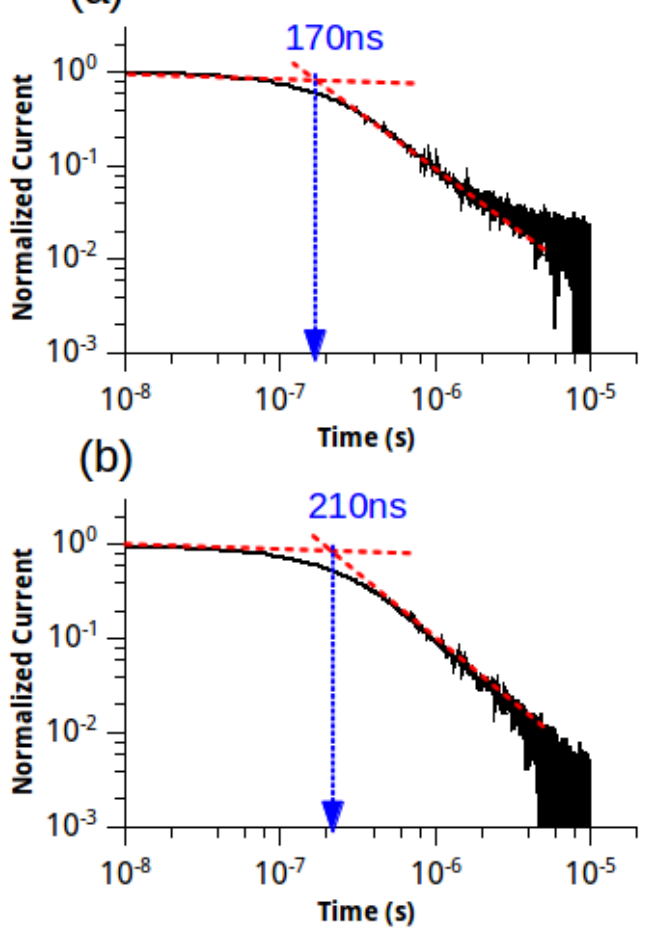

Figure 3.4: Transient photocurrent decay under $-2 V$ reverse bias for (a) P3HT:PCBM and (b) P3HTT-TP:PCBM organic solar cells. The photocurrent is normalized by its initial value at $t=0$.

Figure 3.4(a) and Figure 3.4 (b) plot the transient photocurrent decay of P3HT:PCBM and P3HTT-TP:PCBM cells under $-2 \mathrm{~V}$ reverse bias in log-log scale, respectively. The transit time is determined by the intersection of two "linear" fit lines in the initial decay region and the long tail. The transit time for the P3HT:PCBM cell is $170 \mathrm{~ns}$ and that for the P3HTT-TP:PCBM cell is $210 \mathrm{~ns}$. Using Equation ( 3.3) for devices with active layer thickness of $80 \mathrm{~nm}$, these correspond to mobilities of $7.3 \times 10^{-5} \mathrm{~cm}^{2} /(\mathrm{V} \times \mathrm{s})$ and $6.3 \times 10^{-5} \mathrm{~cm}^{2} /(\mathrm{V} \times \mathrm{s})$ for P3HT:PCBM and P3HTTTP:PCBM devices, respectively. Since electron mobility is generally much larger than hole 
mobility in P3HT:PCBM organic solar cells and the difference is even larger for P3HTTTP:PCBM organic solar cells, we conclude that we have measured the electron mobilities in those materials .

Figure 3.5 shows the measured transient photocurrent decay time constant $\left(\tau_{1 / \mathrm{e}}\right)$ as a function of forward applied bias. For the P3HT:PCBM cell $\tau_{1 / \mathrm{e}}$ monotonically increases as the applied bias increases and saturates near $\mathrm{V}_{\mathrm{oc}}$. The trend is less obvious for the P3HTT-TP:PCBM cell, but $\tau_{1 / \mathrm{e}}$ is generally smaller than the P3HT:PCBM cell in the recombination-dominated region (near $\mathrm{V}_{\mathrm{oc}}$ ). The corresponding recombination lifetime is approximately $0.9 \mu$ s for P3HT:PCBM and $0.4 \mu$ s for P3HTT-TP:PCBM cells.

Strong recombination in the P3HTT-TP:PCBM cell, which results in shorter lifetime, will impede charge collection and limit the overall power conversion efficiency. More specifically, the charge collection efficiency $\eta_{\mathrm{cc}}$ can be estimated using the relation

$$
\eta_{c c}=\frac{\tau_{r e c}}{\tau_{r e c}+\tau_{s w}}
$$

where $\tau_{\text {rec }}$ is the recombination lifetime, and $\tau_{\mathrm{sw}}$ is the sweep-out time [15]. The calculated charge collection efficiency is $\sim 80 \%$ for the P3HT:PCBM cell under short-circuit condition, which is larger than the value of $60 \%$ for the P3HTT-TP:PCBM cell. 


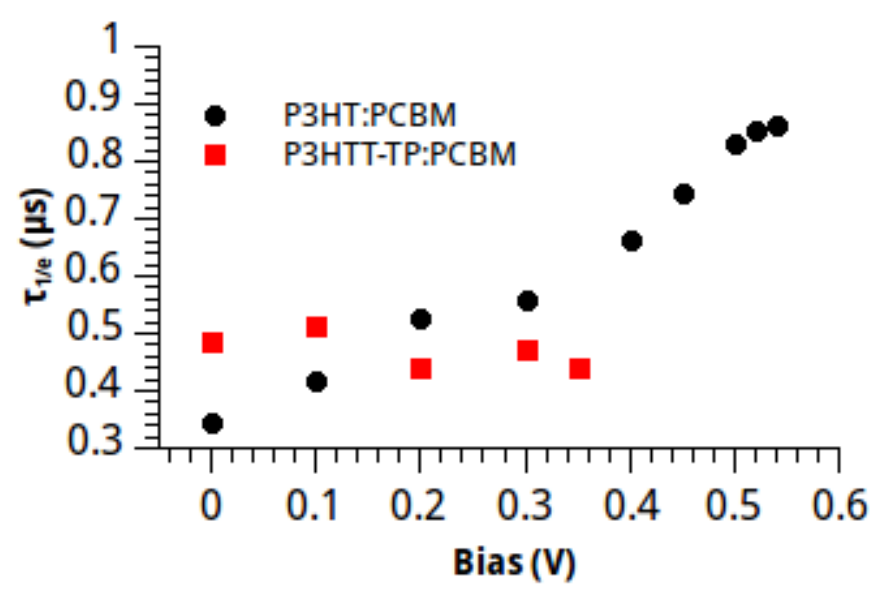

Figure 3.5: Transient photocurrent decay time constant $\tau_{1 / e}$ as a function of the forward applied bias.

\subsection{Summary}

In this chapter, the principles of transient photoconductivity method are described, and the transient photocurrents of BHJ organic solar cells are analyzed. By studying the falling edge of transient photocurrents, we are able to extract the carrier mobilities and recombination lifetimes of P3HT:PCBM and P3HTT-TP:PCBM BHJ organic solar cells. The difference in the mobilities and lifetimes of those two systems is consistent with the difference in the DC characteristics, especially the short-circuit current density and power conversion efficiency. However, the charge collection efficiency of P3HTT-TP:PCBM is not significantly smaller than P3HT:PCBM, while the former has higher absorption than the latter [50]. This indicates that only the huge difference in the exciton diffusion, exciton dissociation or charge transfer can justify the drastic difference in the power conversion efficiency. Further studies are needed to understand those physical processes, but that is beyond the capability of the transient photocurrent method. 


\section{Chapter 4 : Photo-induced Current Transient Spectroscopy}

\subsection{Introduction}

The work in this chapter would not have been possible without the hybrid solar cells generously provided by Matt Greaney in Dr. Richard Brutchey's group at the University of Southern California.

As discussed in Chapter 2, the charge transport in amorphous material systems like organic solar cells is governed by hopping processes, and is heavily influenced by the mid-bandgap energy states or trap states. Knowing the distribution of these trap states not only helps to understand charge transport physics in different amorphous material systems, but also provides a quantitative metric for device optimization.

Hybrid solar cells consisting of organic polymers and inorganic semiconductor nanocrystals are promising alternatives to fullerene-based all-organic solar cells owing to the tuneable band gap, high electron mobility, strong broadband absorption, and modular surface chemistry inherent to semiconductor nanocrystals [19], [20]. The syntheses of the nanocrystals are often carried out in the presence of organic surfactants or ligands, which have several functions, such as directing the growth of the nanocrystals, tuning the highest occupied molecular orbital (HOMO) and lowest unoccupied molecular orbital (LUMO) of the nanocrystals, and passivation of the nanocrystal surface [53]-[55]. These functions can directly impact the power conversion efficiency (PCE) of excitonic solar cells through modulation of the open circuit potential $\left(\mathrm{V}_{\mathrm{OC}}\right)$ [56], [57] and short circuit current [58].

One of the widely used inorganic nanocrystals for hybrid solar cells is cadmium selenide (CdSe) in the form of quantum dots, nanorodes, tetrapods, and hyperbranches [58]-[60]. During the post-synthesis surface mediation of $\mathrm{CdSe}$, small molecules as surface capping ligands are used to replace native ligands in order to facilitate charge transfer between the donor and acceptor [61]. With different surface capping ligands, the performance of CdSe hybrid solar cells varies significantly. Lek et al. found that P3HT:CdSe hybrid solar cells treated with 2thiophenepropionic acid (TPA) have much lower short circuit current density, $\mathrm{J}_{\mathrm{SC}}$, than those treated with 2-cyano-3-(-thiophen-2-yl) acrylic acid (CTAA) [62]. Greaney and coworkers found 
that quantitative displacement of CdSe native ligands with tert-butylthiol (tBT) resulted in an increased CdSe LUMO energy, as determined by a combination of electrochemical techniques [63], [64]. When incorporated into hybrid solar cells with poly(3-hexylthiophene-2,5-diyl) (P3HT), the tBT-treated CdSe exhibited an enhanced $\mathrm{V}_{\mathrm{OC}}$ (up to $0.8 \mathrm{~V}$ ) compared to pyridine (Py)-treated CdSe, which in combination with an increase in $\mathrm{J}_{\mathrm{SC}}$ led to PCEs ranging from 2\%$3 \%$ [64], [65]. Such difference is thought to be resulted from the creation or removal of surface trap states on the nanocrystals during the native ligand exchange process. Thus, knowing the trap state distribution and the emission rates of trap states is very important. To date, different measurement techniques such as time-correlated single photon counting [64] and transient absorption spectroscopy [62] have been employed to analyze the trap states and their impact on charge transport mechanisms. However, there are still very few reports on the energy level landscape in such systems.

Photo-induced current transient spectroscopy (PICTS) is a derivative of deep-level trap spectroscopy (DLTS) [66], and it is one of the few available methods that has been successfully applied to inorganic materials to extract quantitative information about trap levels [67]-[69]. Recently, Neugebauer et al. reported using DLTS on organic solar cells to study the role of trap states and activation energies [70]. We use the PICTS technique to examine the energy spectrum of trap states in hybrid solar cells fabricated from bulk heterojunctions of $\mathrm{P} 3 \mathrm{HT}$ and $\mathrm{CdSe}$ nanocrystals ligand exchanged with either Py or tBT. A data processing workflow to extract the trap emission spectra is implemented by solving an inverse problem with regularization techniques. We show that a correlation exists between the density of trap states and device performance. Such correlation is further corroborated by the mobilities of P3HT:CdSe(Py) and P3HT:CdSe(tBT) solar cells measured by the transient photoconductivity method.

\subsection{Principles of PICTS}

Different from the trapping model based on Gaussian or exponential density of states, the trap sites studied by PICTS are assumed to be made up of a series of discrete levels as shown in 
Figure 4.1. Two correlated processes, trapping and detrapping between the trap levels and mobility edges (HOMO and LUMO), happen randomly.

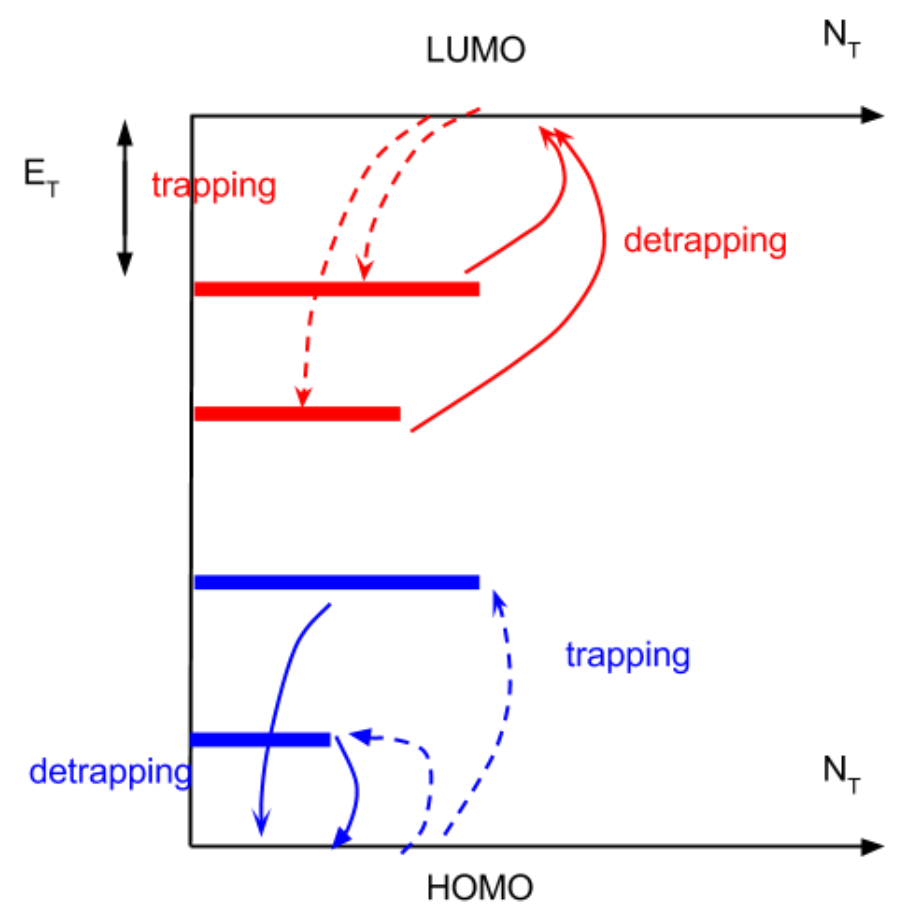

Figure 4.1: The trapping model in semiconductors for PICTS method.

For PICTS measurements, a cell is excited by a light pulse and the transient photocurrent is recorded. Once the light is switched off, after the initial carrier sweep-out, the primary contribution to the photocurrent tail is the emission of carriers that were trapped during photoexcitation. To simplify the complicated underlying physics of transient photocurrents, it is assumed that carrier retrapping and recombination is negligible in the later part of the photocurrent tail when carrier concentrations are low. A multi-exponential photocurrent decay is expected if there are several active trap levels. Without assuming specific details about the trap levels, the transient photocurrent can be described by the expression [71]

$$
I(t)=C \int_{0}^{\infty} N_{T}(f) f \exp (-f t) d f
$$


where $\mathrm{f}$ is the emission rate of trapped carriers $\left(\mathrm{s}^{-1}\right), \mathrm{N}_{\mathrm{T}}$ is the trap emission spectrum as a function of emission rate $\left(\mathrm{s} \times \mathrm{m}^{-3}\right)$, and $\mathrm{C}$ is a proportionality factor $\left(\mathrm{A} \times \mathrm{s} \times \mathrm{m}^{3}\right)$. In the case of a thermally activated detrapping process, the trap emission rate, activation energy, and temperature are related via[71]

$$
f=f_{0} \exp \left(-\frac{E_{T}}{k T}\right)
$$

where $E_{T}$ is the activation energy $(\mathrm{eV}), \mathrm{k}$ is the Boltzmann constant $\left(\mathrm{eV} \times \mathrm{K}^{-1}\right), \mathrm{T}$ is the temperature $(\mathrm{K})$, and $\mathrm{f}_{0}$ is a proportionality factor $\left(\mathrm{s}^{-1}\right)$. For a crystalline inorganic semiconductor, $\mathrm{f}_{0}=\sigma \mathrm{v}_{\mathrm{th}} \mathrm{N}_{\mathrm{c}, \mathrm{v}}$, where $\sigma$ is the capture cross section $\left(\mathrm{m}^{2}\right), \mathrm{v}_{\text {th }}$ is the carrier thermal velocity $\left(\mathrm{m} \times \mathrm{s}^{-}\right.$ ${ }^{1}$ ), and $\mathrm{N}_{\mathrm{c}, \mathrm{v}}$ is the effective density of states in the conduction band or the valence band $\left(\mathrm{m}^{-3}\right)$. In the framework of the Miller-Abrahams hopping model, which is the model used herein, the proportionality factor $\mathrm{f}_{0}$, known as the attempt-to-escape frequency, is considered to be temperature independent[70], [72], [73].

The trap emission spectra can be obtained by solving a least square problem based on Equation ( 4.1) with non-negativity constrained Tikhonov regularization using cross-validation for model selection as illustrated in Figure 4.2, which will be discussed in the next section. We can extract the trap emission peaks from photocurrent transients measured at different temperatures, and determine dominant activation energies by fitting Equation ( 4.2) in Arrhenius plots.

\subsection{Data Processing of PICTS}

The data processing of PICTS is essentially solving an inverse problem which finds the spectral response in emission rate (with the unit of frequency) domain from the transient signal in the time domain, as described by Equation (4.1). However, this is a non-trivial process. 


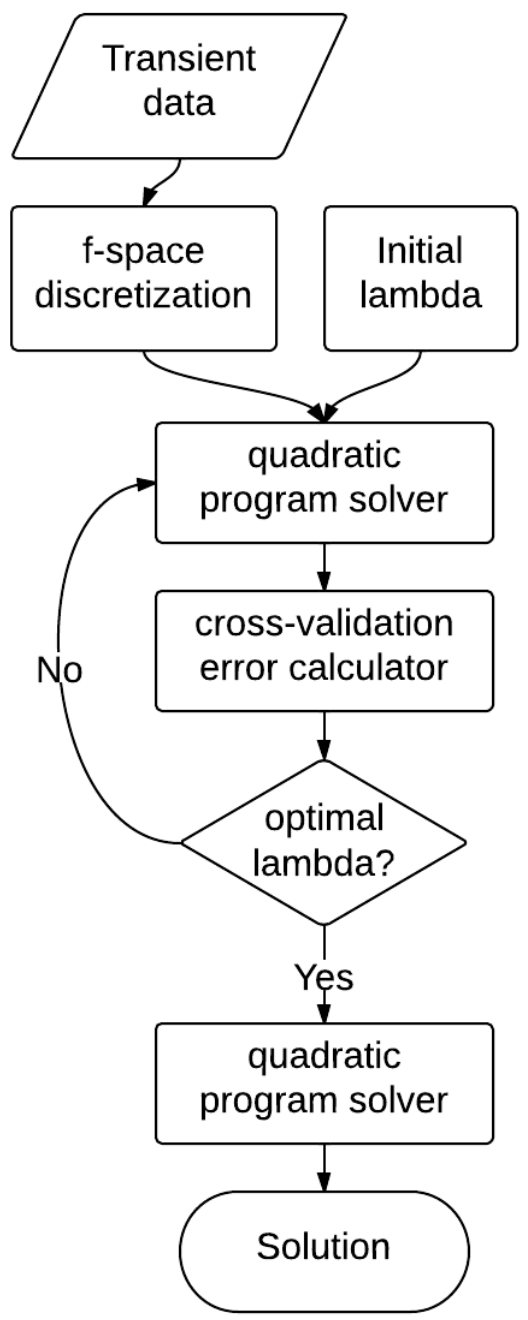

Figure 4.2: The PICTS data processing workflow.

\section{Discretization}

The first step is the discretization of the right-hand side of Equation (4.1). Owing to its high dynamic range, we discretize f-axis logarithmically into $f_{i}\left(i=1 \ldots N_{f}\right.$, ) as

with

$$
\begin{gathered}
f_{i}=f_{\text {min }} \theta^{i-1} \\
\theta=\left(\frac{f_{\text {max }}}{f_{\text {min }}}\right)^{\frac{1}{N_{f}-1}}
\end{gathered}
$$


where $f_{\max }$ and $f_{\min }$ are the upper and lower bounds of the $f$ grid, and $\mathrm{N}_{\mathrm{f}}$ is the number of discretization points.

\section{Regularization}

Contrary to the simplistic look of Equation ( 4.1) which is commonly known as the Fredholm integral equation of the first kind, obtaining the spectrum $\mathrm{N}_{\mathrm{T}}(\mathrm{f})$ from it is not trivial. The illposed nature of the inverse problem defined by Equation (4.1) makes the solution vulnerable to noise if only direct matrix inversion is used [74]. This is always a problem because experimental data contain noise. Here we use a regularization technique that damps the influence of noise while maintaining reasonable fits to the data. With the assumption that the optimal and most physically meaningful solution provides the best fit with the minimal fluctuation of the obtained spectrum from the inverse transform, we choose the widely used Tikhonov regularization of zero-order (regularization matrix being an identity matrix), and transform the problem to finding the optimal $\mathrm{N}_{\mathrm{T}}(\mathrm{f})$ that minimizes the objective function $\mathrm{h}\left[\mathrm{N}_{\mathrm{T}}(\mathrm{f})\right]$ as follows:

$$
h\left[N_{T}(f)\right]=\sum_{t}\left|\int_{0}^{\infty} N_{T}(f) f \exp (-f t) d f-I(t)\right|^{2}+\lambda^{2}\left|L_{T}(f)\right|^{2}
$$

where $\lambda$ is the regularization parameter and $\mathrm{L}$ is penalization matrix. Here $\mathrm{L}$ is an identity matrix which has been widely used in previous work [68]. Note that the first term on the right-hand side of Equation ( 4.4) is the residue of the fit, and the second term penalizes the roughness of the solution. At the same time, a physically meaningful $\mathrm{N}_{\mathrm{T}}$ is non-negative, which imposes a constraint on the solution. Therefore, the mathematical problem to be solved can be written in the form of a typical constrained quadratic programming $(\mathrm{QP})$ problem as

Minimize: $\mathrm{h}\left[\mathrm{N}_{\mathrm{T}}(\mathrm{f})\right]$

Subject to: $\mathrm{N}_{\mathrm{T}}(\mathrm{f}) \geq 0$.

Once $\lambda$ is given, the problem described in Equation ( 4.5) can be solved using a standard QP solver.

\section{Regularization parameter selection}


Since the value of the regularization parameter $\lambda$ directly impacts the final solution $\mathrm{N}_{\mathrm{T}}(\mathrm{f})$, it has to be chosen with caution. If $\lambda$ is too large, the solution may be smooth but may not fit the experimental data well. If $\lambda$ is too small, the solution may have a good fit to the data but the noise may dominate, making the solution physically meaningless. Without prior knowledge about the solution and the noise, "heuristic" parameter selection algorithms are popular choices, and L-curve criterion and cross-validation are two of the widely used methods:

1) L-curve criterion. This method was proposed and popularized by Hansen [75], [76]. It aims to balance the residue of fitting (the first term in Equation (4.4)) and the solution roughness (the second term in Equation ( 4.4)) by an empirical rule. By sweeping the regularization parameter $\lambda$ from a small value to a large value, a set of solutions can be obtained by solving the problem described in Equation (4.5). Consequently, we can calculate the corresponding residue of fitting and solution roughness associated with each $\lambda$. When these two quantities are plotted in log-log scale, the so-called "L-curve" is obtained because of its L-shape. The L-curve criterion selects the $\lambda$ at the knee of the Lcurve as the trade-off of those two quantities. A graphical representation of L-curve criterion is illustrated in Figure 4.3. Hansen demonstrated that finding the knee point of the L-curve could be very fast for unconstrained problems due to its unique properties [76]. However, this method, although it performs well for certain problems [75], is very empirical and still lacks solid mathematical foundations.

2) Ordinary cross-validation is a widely used method for model selection which can also be applied to calculate the optimal $\lambda$ in regularization. The basic idea is to use a subset of the data to "cross-validate" the model trained by the other part of the data, and search for the optimal $\lambda$ corresponding to the minimum averaged cross-validation error. If there are $\mathrm{N}$ data points in total, $\mathrm{k}$-fold cross-validation means the whole data set is divided into $\mathrm{k}$ subparts, and one subpart is used to test the model built by the other (k-1) subparts in a single iteration. Therefore, $\mathrm{k}$-fold cross-validation is also called leave-(N/k)-out crossvalidation since there are $\mathrm{N} / \mathrm{k}$ points left out when building the model each time. It is easy to find that there will be $\mathrm{N} \times \mathrm{k}$ iterations in total for an ordinary cross-validation calculation. The ordinary cross-validation procedure is as follows:

a) Generate $\lambda$ grid $\left[\lambda_{1}, \lambda_{2}, \ldots, \lambda_{N}\right]$.

b) In $\mathrm{j}^{\text {th }}$ iteration of each $\lambda_{\mathrm{i}}$, for each $\mathrm{p}_{\mathrm{j}} \in\left(\mathrm{p}_{1}, \mathrm{p}_{2}, \ldots, \mathrm{p}_{\mathrm{k}}\right)$ where $\mathrm{p}_{\mathrm{j}}$ is the a data subset, 
compute the solution with the model without data subset $\mathrm{p}_{\mathrm{j}}$. And then compute the cross-validation error $\epsilon_{\mathrm{j}}$ by testing the solution with data subset $\mathrm{p}_{\mathrm{j}}$ for this iteration.

c) Compute the averaged cross-validation error for this $\lambda_{\mathrm{i}}$ with the Equation ( 4.6).

$$
\epsilon_{C V}\left(\lambda_{i}\right)=\frac{1}{k} \sum_{j=1}^{k} \epsilon_{j}\left(\lambda_{i}, p_{j}\right)
$$

d) Proceed to the next $\lambda_{i}$, and plot the averaged cross-validation error against $\lambda$ grid. Find the optimal $\lambda$ corresponding to the minimum error.

A graphical demonstration of 5-fold ordinary cross-validation for our PICTS data processing can be found in Figure 4.4 .

Here we use ordinary cross-validation thanks to the suggestion from Dr. Daniel Weller.

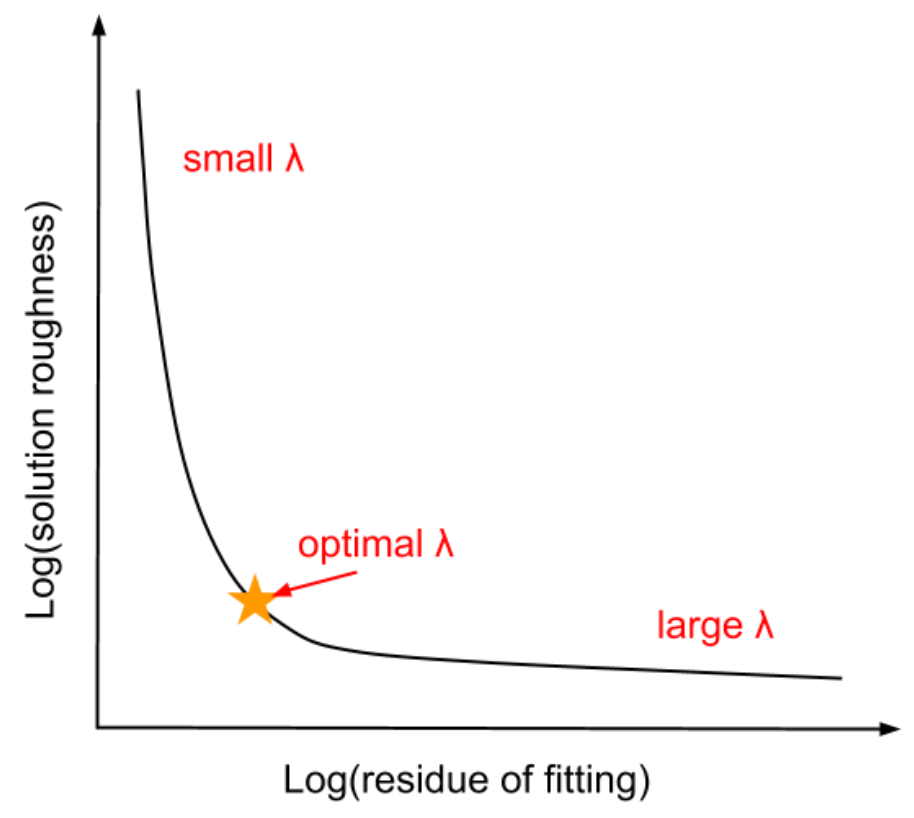

Figure 4.3: A graphical demonstration of the principle of L-curve criterion. $\lambda$ is the regularization parameter. 
(a)

$$
\begin{aligned}
& \begin{array}{|l|l|l|l|l|}
\hline \text { test } & \text { train } & \text { train } & \text { train } & \text { train } \\
\hline
\end{array} \\
& \text { CV error }=\frac{1}{n} \sum\left\|C_{\text {test }}(t)-\int_{0}^{\infty} N_{\text {Ttrain }}\left(f_{\text {train }}\right) \exp \left(-f_{\text {train }} t_{\text {test }}\right) d f_{\text {train }}\right\|_{2}^{2}
\end{aligned}
$$

(b)

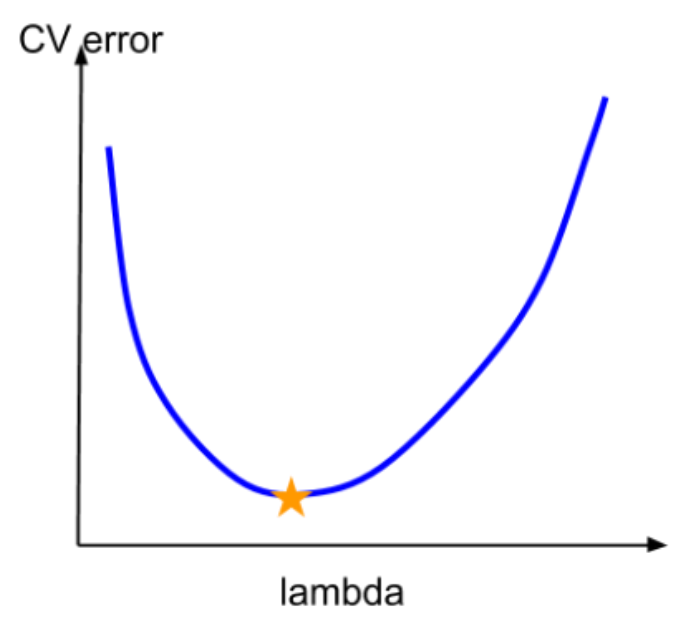

Figure 4.4: A graphical demonstration of 5-fold ordinary cross-validation for PICTS data processing.

\section{Numerical test}

To show the effectiveness of the workflow, we ran a numerical test as follows. An artificial trap emission spectrum consisting of three discrete delta-function peaks Table 4.1 was generated. The corresponding noise-free transient current was calculated using Equation (4.1), and white noise was superimposed on the noise-free transient, as illustrated in Figure 4.5(a). The solution as shown Figure 4.5(b) was obtained after applying 10-fold cross-validation. After fitting the peaks with Gaussian profiles, the center frequencies and the areas under each peak were computed; the results were also tabulated in Table 4.1. The center frequencies of the solution were close to the original input, with the slowest component being the most error-prone due to the influence of the noise. The areas under each peak corresponded to the peak strength. 
Table 4.1: The parameters of the input artificial spectrum and the solution. $f_{c}$ and $A$ are the center frequencies and areas of the peaks.

\begin{tabular}{|l|l|l|l|l|}
\hline \multicolumn{2}{|l|}{ Peak \# } & 1 & 2 & 3 \\
\hline \multirow{2}{*}{$\begin{array}{l}\text { Artificially generated } \\
\text { input }\end{array}$} & $\mathrm{f}_{\mathrm{c}}$ & 1 & 0.3 & 0.1 \\
\cline { 2 - 5 } & $\mathrm{A}$ & 3 & 1 & 0.5 \\
\hline \multirow{2}{*}{\begin{tabular}{l} 
Solution (Figure 4.5 (b)) \\
\cline { 2 - 5 }
\end{tabular}} & $\mathrm{f}_{\mathrm{c}}$ & 0.99 & 0.27 & 0.08 \\
\cline { 2 - 5 } & $\mathrm{A}$ & 3.05 & 1.05 & 0.43 \\
\hline
\end{tabular}

(a)

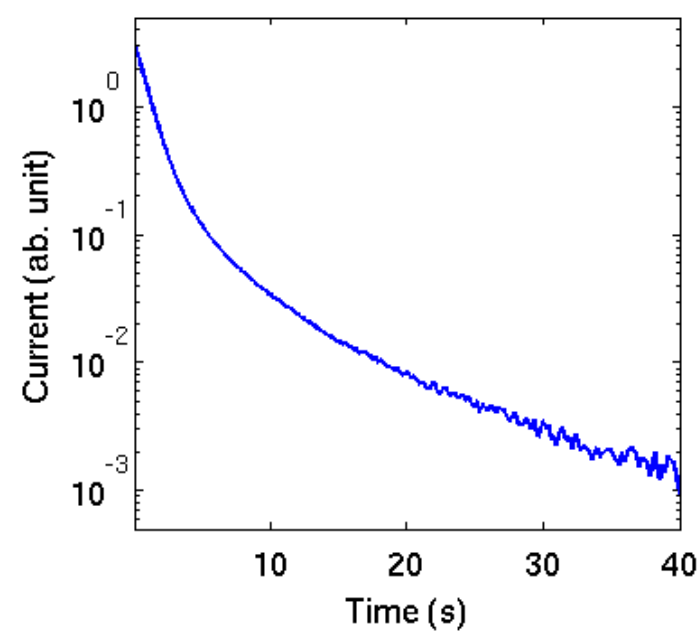

(b)

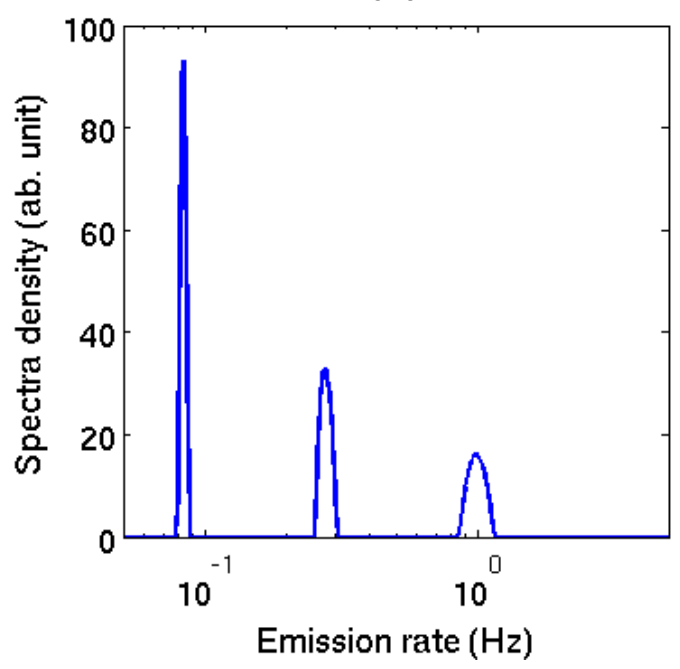

Figure 4.5: (a) The transient current corresponding to the artificially generated spectrum in Table 4.1but with additive white noise. (b) The solution to the inverse transform of the transient current in (a) with 200 sampling points in $f$-space.

\subsection{Experiments and Discussion}

The synthesis of CdSe nanocrystals, the Py and tBT ligand exchange, and device fabrication are documented elsewhere [64]. The DC characteristics of P3HT:CdSe(tBT) and P3HT:CdSe(Py) cells are listed in Table 4.2. 
Table 4.2: The open-circuit voltage $\left(V_{o c}\right)$, short-circuit current density $\left(J_{\text {sc }}\right)$, fill factor $(F F)$ and power conversion efficiency (PCE) of P3HT:CdSe(tBT) and P3HT:CdSe(Py) hybrid solar cells. The device parameters were measured under AM1.5G illumination at 1 sun.

\begin{tabular}{|l|l|l|l|l|}
\hline & $\mathrm{V}_{\mathrm{oc}}(\mathrm{V})$ & $\mathrm{J}_{\mathrm{sc}}\left(\mathrm{mA} \cdot \mathrm{cm}^{-2}\right)$ & FF & PCE (\%) \\
\hline P3HT:CdSe(tBT) & 0.74 & 6.6 & 0.54 & 2.6 \\
\hline P3HT:CdSe(Py) & 0.69 & 4.2 & 0.50 & 1.5 \\
\hline
\end{tabular}

For PICTS measurements, a cell was placed inside a liquid-nitrogen-cooled, continuous-flow cryostat. There were two temperature sensors: one was used to monitor the temperature of the cryogenic base underneath the sample substrate, and the other was placed next to the device being measured. The measured temperature variation of the device was less than $0.3 \mathrm{~K}$ over a period of $0.5 \mathrm{~h}$. Although a large reverse bias was preferred to minimize the influence of recombination on the transient photocurrent tail [77], the devices were measured at short-circuit condition to minimize the potential long-term electrical instability of P3HT:CdSe devices and suppress the dark current floor. Electrical pulses with repetition rate of $0.2 \mathrm{~Hz}$ and pulse width of $3 \mathrm{~ms}$ were generated from an Agilent 81110A pulse generator and used to produce optical pulses from a $405-\mathrm{nm}$ laser diode. The low pulse duty cycle ensured that the cell recovered to equilibrium after each excitation. The photocurrent was driven to a relatively flat plateau by the optical pulse to ensure enough trap filling before the decay. The photocurrent decay was measured when the laser was switched off. The photocurrents were amplified by an SR570 lownoise preamplifier and were recorded by a National Instrument USB-6211 data acquisition unit. Measurements were performed from $200 \mathrm{~K}$ to $250 \mathrm{~K}$. To minimize the influence of the initial sweep-out and recombination currents, which usually finished after several microseconds for similar material systems such as poly(3-hexylthiophene-2,5-diyl):6,6-phenyl-C61-butyric acid methyl ester (P3HT:PCBM) [46], [78], only the transient data later than $0.01 \mathrm{~ms}$ after the falling edge were processed. The end point of the time window was limited by either the signal-to-noise ratio or the repetition rate. The current reached the noise floor of the experimental setup after several hundred milliseconds and was limited by the Johnson noise, which was primarily due to the finite shunt resistance of the hybrid solar cells. To ensure a well-conditioned matrix during the inverse transform, we sampled points logarithmically equidistant in time scale [79], [80] for each temperature. 
A very similar setup was used for the transient photoconductivity measurement at room temperature. The device was biased at $-3 \mathrm{~V}$, and was photoexcited by $200-\mathrm{ns}$ wide 405 -nm laser pulses with $20 \mathrm{~Hz}$ repetition rate. The fast transient photocurrent decay was recorded by a highspeed Agilent DSO81004B oscilloscope that had 50-Ohm input impedance. For these measurements the device area was reduced to remove $\mathrm{RC}$ time constant effects on the transients.

Figure 4.6 (a) shows four typical PICTS transient photocurrents. The system RC time constant is no larger than $0.5 \mu \mathrm{s}$, and thus should not be a limiting factor in the photocurrent decay shown in Figure 4.6 (a). While the level of photocurrents is related to both carrier mobility (which is also a function of temperature) and the detrapping rate, the decay rate of the photocurrent tails is a stronger indicator of the detrapping rate and less correlated to the carrier mobility in our PICTS model. For both the P3HT:CdSe(tBT) and P3HT:CdSe(Py) devices, the photocurrent tails decay faster at higher temperatures. This is not surprising considering the detrapping rate decreases with temperature according to Equation (4.2). A closer look at all transients in Figure 4.6 reveals that the initial fast decay components correspond to Peak \#1 in Figure 4.6 (c) and Figure 4.6 (d). However, the fast initial decay component is less obvious in $243.5 \mathrm{~K}$ because the adjacent slower decay component, Peak \#2 in Figure 4.6(d), is faster at $243.5 \mathrm{~K}$ compared to $198.7 \mathrm{~K}$. On the other hand, when compared at similar temperatures, the photocurrent tails of the P3HT:CdSe(Py) device decay slower than P3HT:CdSe(tBT), an indication of deeper levels in P3HT:CdSe(Py). Generally, it is intuitive to understand and correlate the qualitative features of transient photocurrent decays to material properties. A more quantitative understanding can be obtained by applying PICTS analysis, which we discuss in further detail later. 
(a)

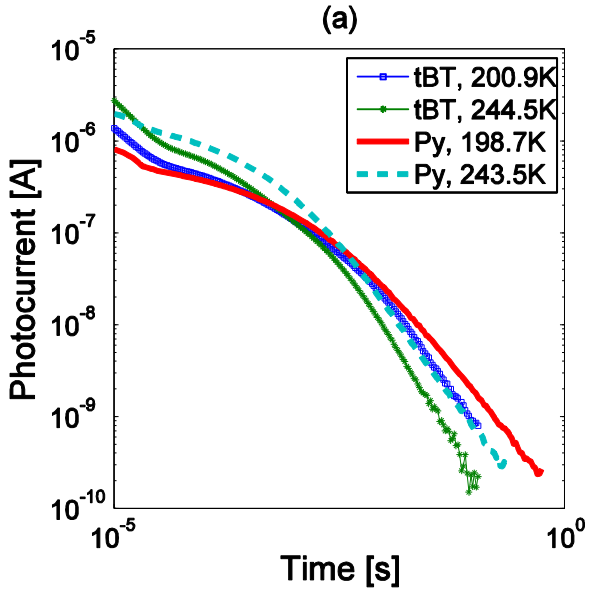

(c)

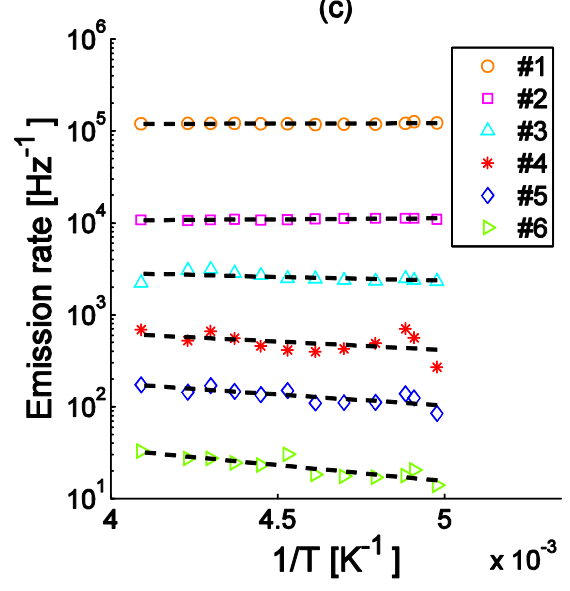

(b)

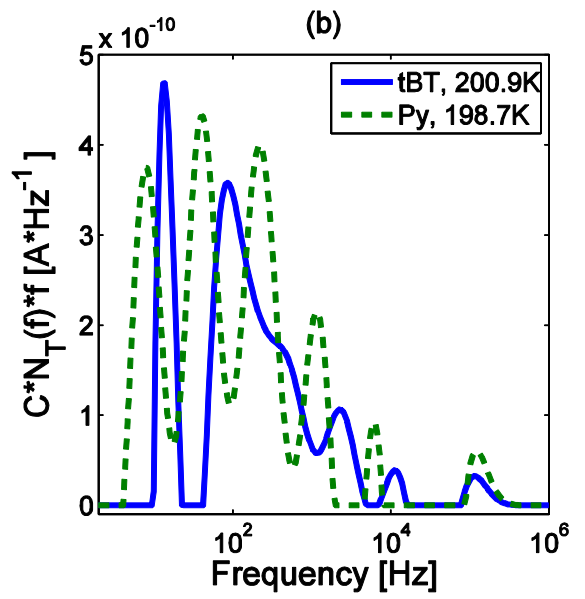

(d)

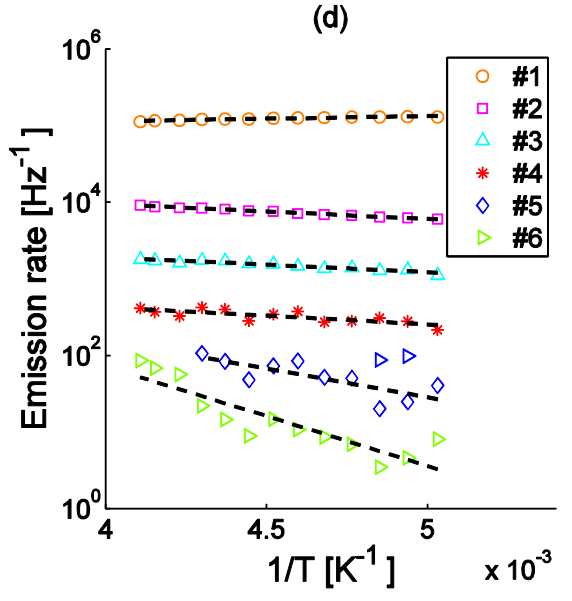

Figure 4.6: (a) Typical PICTS transient photocurrents; (b) typical trap emission spectra after the inverse transform of transients; (c) Arrhenius plot of P3HT:CdSe(tBT); (d) Arrhenius plot of P3HT: $\mathrm{CdSe}(\mathrm{Py})$.

Figure 4.6 (b) shows two typical processed trap emission spectra. The strength of the peaks in Figure 4.6 (b) is defined as $\mathrm{C} \int \mathrm{N}_{\mathrm{T}}(\mathrm{f}) \mathrm{df}$, where $\int \mathrm{N}_{\mathrm{T}}(\mathrm{f}) \mathrm{df}$ is the trap density and $\mathrm{C}$ is a proportionality factor in Equation (1), which can be obtained by calculating the area under each peak. The peak strength is plotted versus peak number in Figure 4.7 (a). Although the absolute values of trap densities are not determined, we can still compare the relative trap densities among different trap levels in a single system. It can be seen that the three deepest levels are more prominent than the shallow levels in term of trap densities for both P3HT:CdSe(Py) and P3HT:CdSe(tBT) systems. The spectrum broadening around each peak (between $40-100 \mathrm{meV}$ ) is fitted with a Gaussian distribution and is plotted in Figure 4.7 (b). 
The emission peaks and their fitting are shown in the Arrhenius plots in Figure 4.6 (c) and Figure $4.6(\mathrm{~d})$. The trap activation energies $\mathrm{E}_{\mathrm{T}}$ can be estimated with least square linear fitting on Equation (4.2). The values of $\mathrm{E}_{\mathrm{T}}$ determined by the method are summarized in Table 4.3. The fitting error $(\delta \mathrm{E})$ associated with the activation energies is also estimated with a $\mathrm{t}$-distribution with $95 \%$ confidence interval, assuming that the noise of each data point in Figure 4.6 (c) and Figure 4.6 (d) is normally distributed. Note that the $\delta \mathrm{E}$ in Table 4.3, only an indicator of the fitting, should not be confused with the energetic broadening to be discussed later.

Table 4.3: The trap activation energies $\left(E_{T}\right)$ and its deviation ( $\left.\delta E\right)$ of P3HT:CdSe(tBT) and P3HT:CdSe(Py) hybrid solar cells extracted from the Arrhenius plots in Figure 4.6 (c) and Figure 4.6 (d). Some $E T$ and $\delta E$ values are not given because they are too shallow to be recognized. Note that $\delta E$ only represents how far the fitted results can be from the "real" values, not the energetic broadening.

\begin{tabular}{|c|c|c|c|c|}
\hline \multirow{2}{*}{ Peak \# } & \multicolumn{2}{|l|}{ P3HT:CdSe(tBT) } & P3HT:CdSe(Py) \\
\cline { 2 - 5 } & ET $(\mathrm{meV})$ & $\delta \mathrm{E}(\mathrm{meV})$ & $\mathrm{ET}(\mathrm{meV})$ & $\delta \mathrm{E}(\mathrm{meV})$ \\
\hline 1 & - & - & 39 & 1 \\
\hline 2 & - & - & 39 & 4 \\
\hline 3 & 17 & 10 & 46 & 12 \\
\hline 4 & 36 & 26 & 149 & 47 \\
\hline 5 & 48 & 14 & 258 & 44 \\
\hline 6 & 69 & 14 & - & 12 \\
\hline
\end{tabular}

The trap densities (proportional to peak strength) and energetic broadening are described by the visualization in Figure 4.8. The first peaks in both hybrid systems are too shallow to be confidently analyzed using PICTS, and they may possibly be an artifact of the free carrier sweepout at early timescales, which shows much weaker temperature dependence than the trapping and detrapping processes. A stark difference in trap state energies is observed for the two deepest lying levels in the two hybrid systems under investigation. The deepest traps in the P3HT:CdSe(Py) system are 100-200 meV deeper than the analogous levels observed in the P3HT:CdSe(tBT) system. This indicates that one major effect of the tBT ligand is the passivation of deep-level traps that are otherwise observed for P3HT:CdSe(Py). The conclusion is self- 
consistent with the fact that $\mathrm{P} 3 \mathrm{HT}: \mathrm{CdSe}(\mathrm{tBT})$ demonstrates superior photovoltaic performance than P3HT: $\mathrm{CdSe}(\mathrm{Py})$ as shown in Table 4.2.

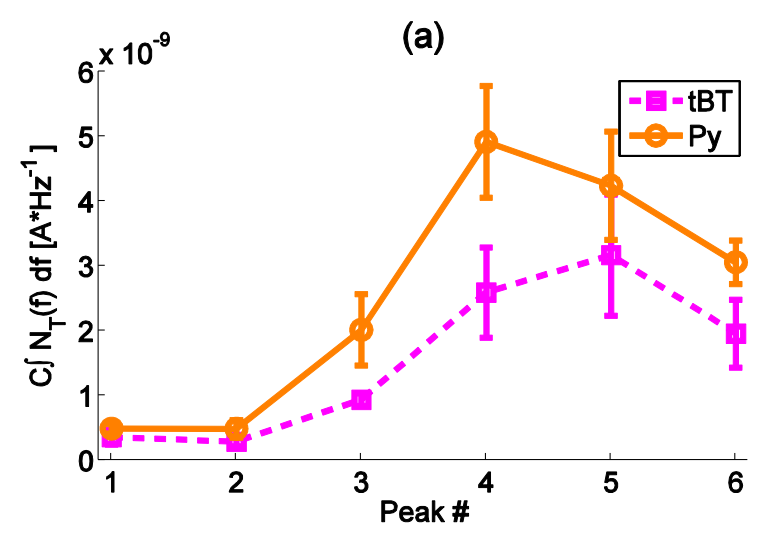

(b)

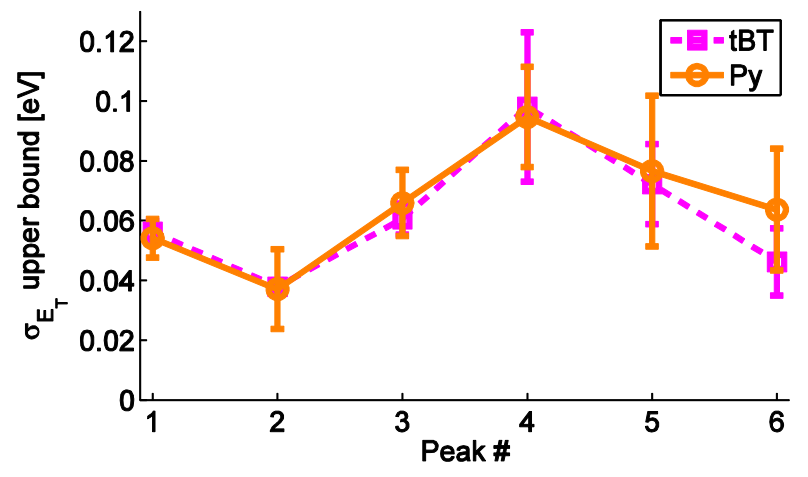

Figure 4.7: (a) Trap emission strength and (b) energetic broadening $\left(\sigma_{E_{T}}\right)$ of P3HT:CdSe(tBT) and P3HT:CdSe(Py) devices.

Transient photoconductivity measurements were performed on the same solar cells for the purpose of comparing the carrier mobilities, and the results are shown in Figure 4.9 and Table 4.4. Based on the PICTS analysis, it would be expected that P3HT:CdSe(tBT) devices exhibit an enhanced mobility relative to $\mathrm{P} 3 \mathrm{HT}: \mathrm{CdSe}(\mathrm{Py})$ devices. The carrier mobility of P3HT:CdSe(tBT) devices is measured to be $1 \times 10^{-4} \mathrm{~cm}^{2} \times \mathrm{V}^{-1} \times \mathrm{s}^{-1}$ compared to $3 \times 10^{-5} \mathrm{~cm}^{2} \times \mathrm{V}^{-1} \times \mathrm{s}^{-1}$ for P3HT:CdSe(Py) devices. We attribute the lower carrier mobility in P3HT:CdSe(Py) bulk heterojunctions to the deeper trap state levels or enhanced energetic disorder, which is consistent with well-known mobility models such as Gaussian disorder model[81] and correlated disorder model[82], [83]. 
However, the physical interpretation of the mobilities measured by transient photoconductivity of our P3HT:CdSe devices is not straightforward and conclusive. In the traditional time-of-flight measurement with transient photoconductivity, the film thickness is much larger than the absorption depth of the incident light so that most of the photogeneration occurs only in one end of the film, and the type of carrier associate with the measured mobility can be easily distinguished [84], [85]. However, this assumption is not valid for thin-film systems such as the P3HT:CdSe cells studied here. Rather, photogeneration occurs throughout the film owing to its thickness of only $\sim 100 \mathrm{~nm}$. Therefore, the contributions to the total photocurrent decay by electrons and holes may not be easily separated unless there is a significant difference in the magnitude of the mobilities. Cowan et al. assumed a large difference in the magnitude of the mobilities of the fast and slow carriers, and extracted mobilities by fitting the initial and later parts of a single photocurrent decay curve [46]. However, as stated previously, such assumption may not hold if the film is not optically thick, and the fitting of the slow carrier may be subjected to the influence of the noise floor. At the same time, Couderc et al. used transient photoconductivity to extract the mobilities of electrons and holes in P3HT:CdSe systems by measuring optically thick films [85]. However, it is well-known that the change in the thickness of an organic film induces change in the film morphology, making the mobility dependent on the film thickness [86]. Therefore the mobilities measured by Couderc et al. may not fully reflect the mobilities in our thin-film P3HT:CdSe systems. Given the difference in the magnitude of mobilities measured by different people and the ambiguity in the type of carriers with which the mobilities are associated [85], [87]-[89], we think that we have extracted the "average" mobility of electrons and holes from two-carrier photocurrent transients. This "average" mobility, although not distinctive by nature, can still reveal the effect of the surface ligands on the properties of the overall material system. 


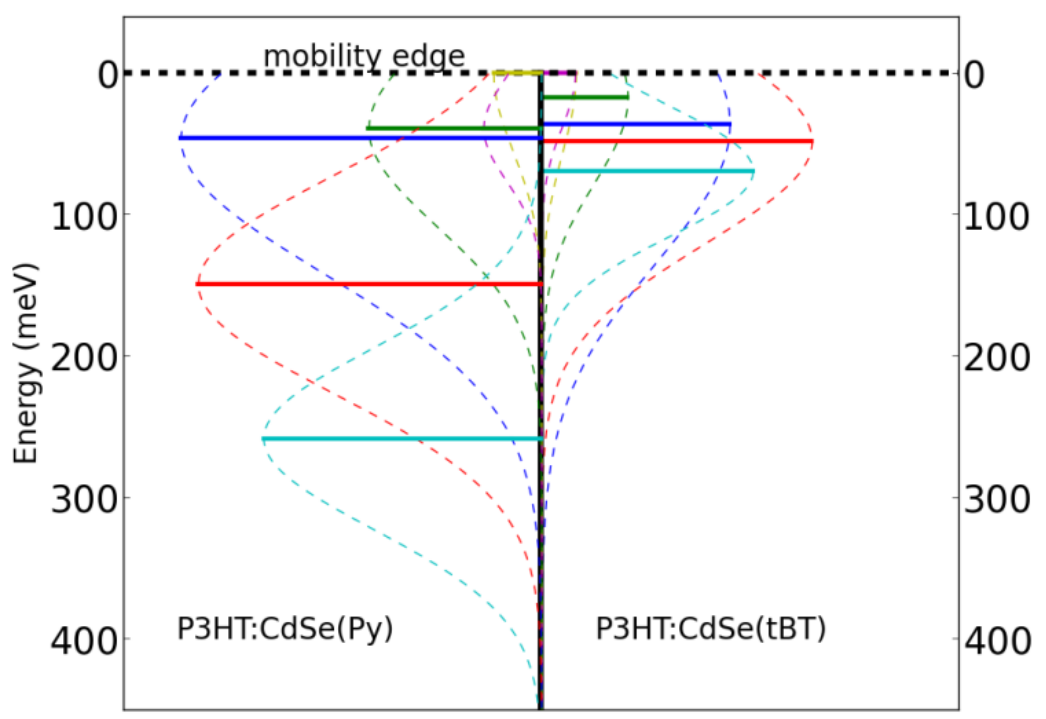

Figure 4.8: The visualization of the trap parameters in Table 4.3 and Figure 4.7.

(a)

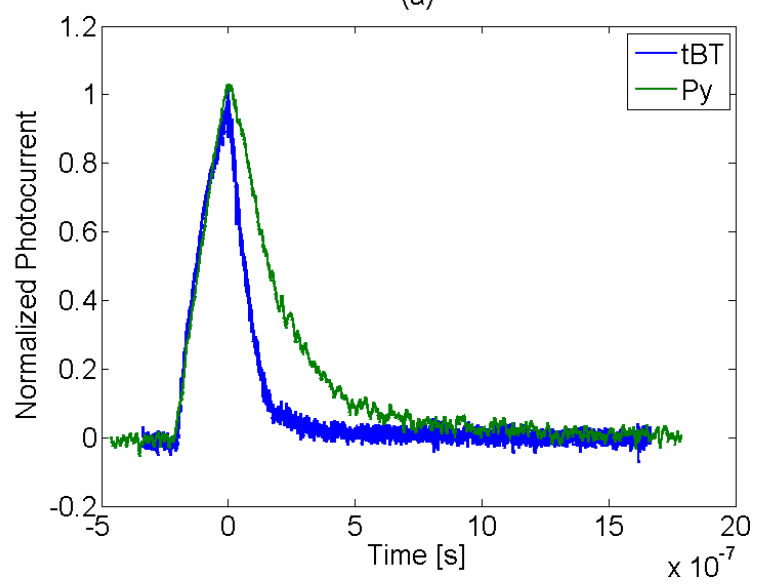

(b)

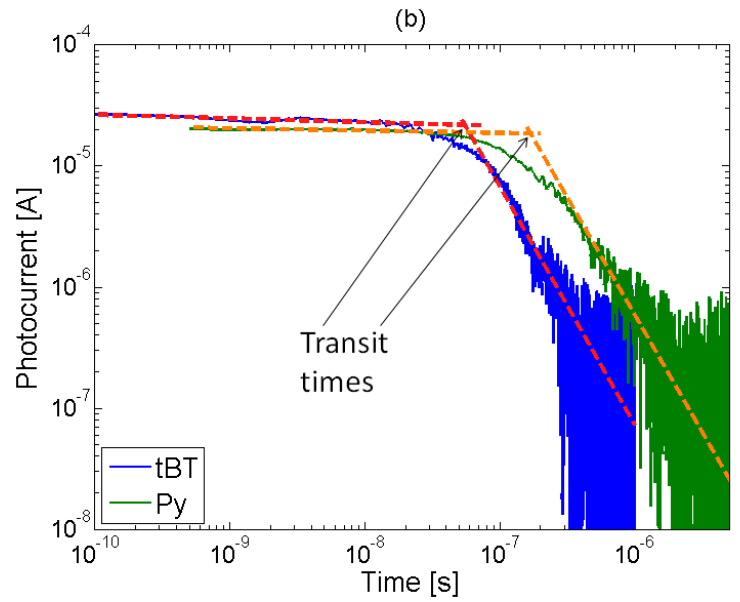

Figure 4.9: (a) Fast photocurrent transient decay normalized to the peak values for P3HT:CdSe(tBT) and P3HT:CdSe(Py); (b) Log-log plots providing a quantitative value of the transit times $\left(t_{t r}\right)$.

As shown in Figure 4.10, we have performed transmission electron microscopy (TEM) analysis on the hybrid films of P3HT:CdSe(Py) and P3HT:CdSe(tBT) to study the film morphology. We can observe clear percolation networks for both systems with minor difference. Both films have also been analyzed with atomic force microscopy (AFM). We see RMS roughness of $1 \mathrm{~nm}$ on the P3HT:CdSe(Py) film and $3 \mathrm{~nm}$ on the P3HT:CdSe(tBT) film. 
Therefore, we have observed difference, although not significant, in the film morphology owing to the different surface ligands. It is known that the film morphology, trap states, and carrier mobility are inter-correlated in an organic thin film [90], [91]. However, the exact physics of such correlation in P3HT:CdSe systems should be investigated with greater details in the future.

Although PICTS can measure trap levels in numerous material systems, it cannot determine whether a trap is donor-type or acceptor-type because the contributions of emitted electrons and holes are indistinguishable in photocurrent analysis. Occasionally, this indistinguishability may give rise to close activation energies with very different emission rates (for example, the $2^{\text {nd }}$ and $3^{\text {rd }}$ peaks in P3HT:CdSe(Py)). One hypothesis is that they belong to different types of traps: one being an electron trap and the other a hole trap. Future work is needed to determine their exact physical properties.

Understanding the trap density of states (DOS) of organic or hybrid solar cells is beneficial owing to its correlation with photovoltaic performance. Typically, the trap DOS is empirically assumed to be exponential or Gaussian in device modeling[92]. Under this assumption, the disordered properties of DOS are manifested by a "dispersive transport" model [47], which can be experimentally characterized by the transient photoconductivity method[48], [77]. Based on this model, it is reported that both open-circuit voltage and short-circuit current are reduced with increased energetic disorder [93]. On the other hand, MacKenzie et al. found localized trap DOS in P3HT:PCBM systems using a combination of transient photocurrent measurements and device modeling [94]. Our work extracts localized DOS from transient photocurrents, with the assumption that DOS is made up of broadened discrete trap levels. Despite the difference in the initial assumption on DOS and quantitative details of data processing from the Gaussian disorder model, we also reach a strong conclusion that is consistent with the DC performance of P3HT:CdSe hybrid solar cells treated with Py and tBT ligands. Thus, this work provides a supplemental perspective for understanding the electronic energy landscape within hybrid solar cells.

Table 4.4: Mobilities of P3HT:CdSe(tBT) and P3HT:CdSe(Py) solar cells extracted by the transient photoconductivity method.

\begin{tabular}{|l|l|l|}
\hline Device & $t_{\text {tr }}(\mathrm{ns})$ & $\mu\left(\mathrm{cm}^{2} \times \mathrm{V}^{-1} \times \mathrm{s}^{-1}\right)$ \\
\hline
\end{tabular}




\begin{tabular}{|l|l|l|}
\hline P3HT:CdSe (tBT) & 55 & $1 \times 10^{-4}$ \\
\hline P3HT:CdSe (Py) & 170 & $3 \times 10^{-5}$ \\
\hline
\end{tabular}

(a)

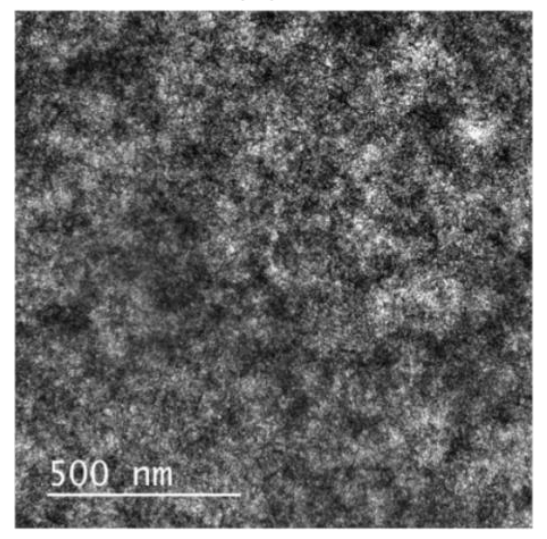

(b)

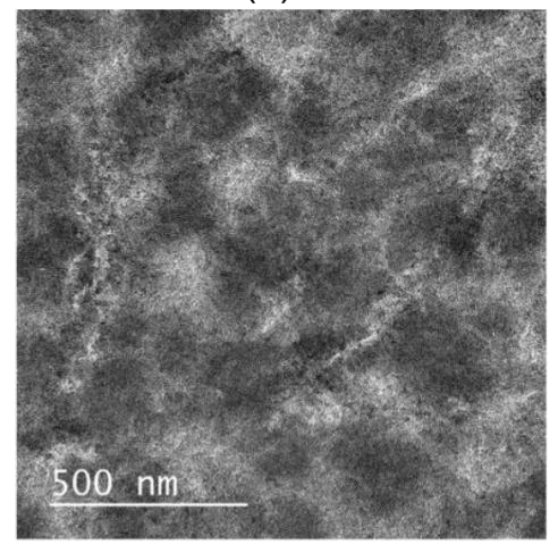

Figure 4.10: TEM images of (a) P3HT:CdSe(Py) and (b) P3HT:CdSe(tBT) BHJ films.

\subsection{Summary}

We have demonstrated the first application of PICTS analysis in hybrid solar cells comparing the effects of two well-utilized CdSe surface ligands on the photocurrent characteristics of P3HT:CdSe hybrid systems. Based on a numerical analysis, we detect four and five trap emission peaks in P3HT:CdSe(tBT) and P3HT:CdSe(Py) systems, respectively. Compared with 
Py treatment, tBT ligand treatment results in the passivation of the deepest trap levels in P3HT:CdSe hybrids. The obtained PICTS results agree with the DC characteristics of P3HT:CdSe(tBT) and P3HT:CdSe(Py) hybrid solar cells and the mobility results extracted by transient photoconductivity. This work demonstrates a useful technique to help understand the energetics of hybrid material systems, and provides physical parameters for device modeling. 


\section{Chapter 5 : Low-Frequency Noise Spectroscopy}

\subsection{Noise in Semiconductors}

Noise is the random fluctuation of signals or their physical representations and is ubiquitous in the universe. It is present in phenomena with very different scales, such as galactic radio waves [95], seismic waves [96], and quantum effect [97]. Noise is usually modeled by random processes and non-deterministic by nature.

Noise has been an active research interest in the community of semiconductor materials and devices for decades. Studying the noise in semiconductors is important and meaningful because:

- Noise limits the quality of signal generation and detection. The inherit noise of semiconductor devices (e.g., BJTs, FETs, diodes, etc.) will impact the performance of active components such as amplifiers, detectors and oscillators. For example, the sensitivity of amplifiers and detectors is directly related to the noise floor, and the timing jitter of high-Q oscillators is determined by their phase noise. Understanding the noise in those semiconductor devices is a very meaningful task in order to make highperformance instruments.

- Noise reflects the underlying physical processes in semiconductor materials and devices. The charge carrier transport plays a central role in the performance of semiconductor devices. Probing the physical parameters such as carrier mobility and lifetime through the noise measurement of the devices provides an invaluable supplemental perspective that complements other characterization techniques.

There are several types of noise associated with semiconductor devices:

1) Thermal noise (Johnson noise or Johnson-Nyquist noise [98], [99]). This type of noise is, as its name suggests, generated by the random thermal agitation of carriers. It is present in any material or device with resistance $\mathrm{R}$. The noise source can be either represented by a voltage source $V_{n}$ or current source $I_{n}$ after Thevenin or Norton equivalence, as shown in Figure 5.1 and Equations ( 5.1) and (5.2)): 


$$
\begin{gathered}
\overline{V_{n}^{2}}=4 k T R \Delta f \\
\overline{I_{n}^{2}}=\frac{4 k T}{R} \Delta f
\end{gathered}
$$

where $\mathrm{k}$ is the Boltzmann constant, $\mathrm{T}$ is the temperature, $\mathrm{R}$ is the source resistance, and $\Delta \mathrm{f}$ is the bandwidth. The noise is "white" in Equations (5.1) and (5.2)) as the spectrum density is independent of the frequency. The whiteness approximation is valid until the frequency is high enough (usually around $\mathrm{THz}$ ) that it becomes necessary to invoke correction by Planck's law, which is not a major concern for the semiconductor devices in this dissertation.

(a)

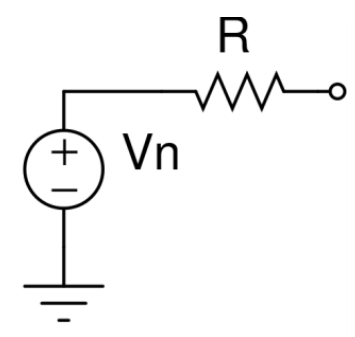

(b)

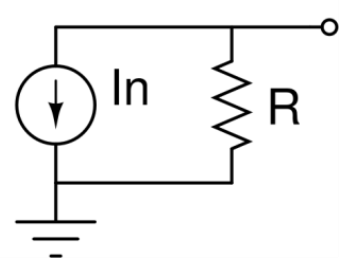

Figure 5.1: (a) Thevenin and (b) Norton representation of noise sources.

2) Shot noise. This type of noise originates from the randomness of carriers that penetrate an energy barrier (e.g., p-n junction) as indivisible units of charge. Therefore, shot noise is only present in junction devices such as diodes or BJTs. The noise can be characterized as a current source as in Equation ( 5.3) 


$$
\overline{I_{n}^{2}}=2 q I_{0} \Delta f
$$

where $\mathrm{q}$ is the unit charge, and $\mathrm{I}_{0}$ is the DC current. It can be seen that shot noise will play a significant role when the current is high.

3) Generation-recombination (G-R) noise. In a semiconductor, a crystal defect can give function as a "generation-recombination center", where the carrier can be randomly captured or released. The fluctuation of the number of carriers during such processes is reflected on the fluctuation of macroscopic quantities such as voltage or current. Thus the corresponding noise is referred to as generation-recombination (G-R) noise, and its spectral density function is in form [100]

$$
S_{n}(f)=\overline{(\Delta N)^{2}} \frac{4 \tau}{1+(2 \pi f \tau)^{2}}
$$

where $\tau$ is the G-R lifetime, and $\overline{(\Delta N)^{2}}$ is the variance of carrier number. For a given G-R center (a given $\tau$ ), the spectral density function is Lorentzian, which is a different from the white noise of thermal noise or shot noise.

4) Random telegraph signal (RTS) noise (or popcorn noise). The time-domain signal of RTS noise looks like a series of binary waves with constant amplitude but random pulse width. Carrier trapping and detrapping by a single trap can cause this. For example, the drain current of a MOSFET may demonstrate RTS behavior even with constant drain-source and gate-source biases, owing to the modulation of carrier numbers in the channel by an oxide trap in an ultrathin gate [101]. The spectral density function of RTS noise is very similar to G-R noise, as shown in Equation ( 5.5) [100]:

$$
S_{n}(f) \propto \frac{I_{0}^{2}}{1+\left(2 \pi f / f_{R T S}\right)^{2}}
$$

where $I_{0}$ is the average current, and $f_{\text {RTS }}$ is the RTS corner frequency which can be further 
written as

$$
f_{R T S}=\frac{1}{\overline{\tau_{l}}}+\frac{1}{\overline{\tau_{h}}}
$$

Here $\bar{\tau}_{l}$ and $\overline{\tau_{h}}$ are the average time of pulse at low and high level.

5) $1 / \mathrm{f}$ noise (flicker noise). $1 / \mathrm{f}$ noise refers to the type of noise with power spectral density inversely proportional to the frequency in the low-frequency region (near DC). Such behavior was translated to the audible "flickering" sound in the radio receiver in the early days of radio and that was the origin of the term "flicker noise". 1/f noise is a ubiquitous phenomenon: it can be found in other completely different situations, such as heart beats, brain waves, stock markets, experimental psychology, and more [102]. Since the origin of $1 / \mathrm{f}$ noise is complicated and its power spectral density may not exactly follow the " $1 / \mathrm{f}$ " trend, a more generalized term, "low-frequency noise" (LFN), is also frequently used. For semiconductor devices such as MOSFETs, although there is still some disagreement on the origin of the LFN, number fluctuation [103], [104] and mobility fluctuation [105][107] are two of the leading theories that can explain significant subsets of the observations thus far.

In the theory of number fluctuation, the number of carriers in the device is modulated by the trapping and detrapping processes associated with crystal defects[103]. This is similar to the underlying physical processes for generation-recombination noise. For example, in MOSFETs, the carriers in the channel can be randomly captured by the defect states in the gate oxide before being released (Figure 5.2 (a)). In a p-n junction diode, similar processes can occur in the space charge region, or near the surface where surface trap states are formed (Figure 5.2 (b)). The power spectral density of the lowfrequency noise owing to number fluctuation will be Lorentzian, which is not " $1 / \mathrm{f}$ " like. However, when there are multiple defects acting as G-R centers, the " $1 / \mathrm{f}$ " signature is formed by the superposition of multiple Lorentzians (similar to (Equation ( 5.4)), which is illustrated in Figure 5.4. When the defects are distributed continuously in the material, the exact " $1 / \mathrm{f}$ " signature is obtained by integrating Equation ( 5.4) in space [103]. Since the carrier trapping and detrapping are often related to the non-idealities in the semiconductor surface, the $1 / \mathrm{f}$ noise generation due to number fluctuation is also referred 
as the "surface effect".

In the theory of mobility fluctuation, as the name suggests, the current fluctuates as a result of fluctuations in the mobility, even if the number of carriers in transport is always constant. The power spectral density is derived by Hooge for a homogeneous media as follows:

$$
S_{n}(f)=\frac{\alpha_{H} I_{D C}^{\gamma}}{f^{\beta} N}
$$

where $\mathrm{I}_{\mathrm{DC}}$ is the $\mathrm{DC}$ current following through the device, $\mathrm{N}$ is the number of carriers participating in the charge transport, $\gamma$ is a constant related to the physical properties of materials and devices, and $\beta$ a constant close to 1 which gives rise to " $1 / \mathrm{f}$ " signature. For inhomogeneous media such as BJTs, MOSFETs or diodes, Equation ( 5.7) does not directly apply and should be reworked. However, in the end it does not change the " $1 / \mathrm{f}$ " behavior of the power spectral density.
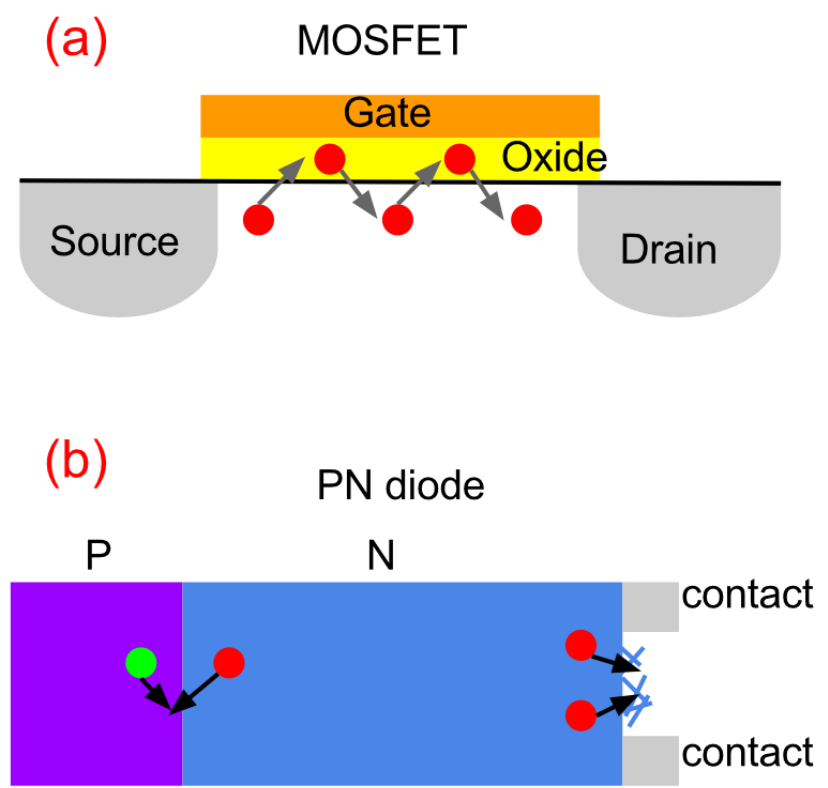

Figure 5.2: Carrier fluctuation caused by the trapping and detrapping in (a) a MOSFET and (b) a diode. 


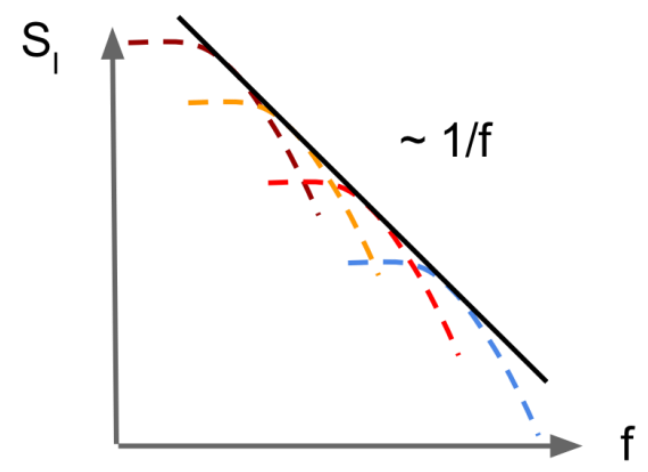

Figure 5.3: The illustration of how G-R noise resulted from multiple defects can give rise to "1/f" signature.

6) Avalanche noise. This type of noise is only present in semiconductor devices that exhibit impact ionization such as avalanche photodiodes or avalanche transistors that operate at very high electric fields. The randomness of the impact ionization events gives rise to excess noise that is different from the other noise types mentioned above. Since devices studied in this dissertation do not operate under such extreme conditions, this noise type is not discussed here.

Understanding the low-frequency noise (LFN) in electronic devices, such as diodes [108], [109] and transistors [110], has been an active topic since the 1950s [103], [104]. The correlation of LFN to material quality and device reliability has also made it a useful non-intrusive characterization tool [111]-[114]. Recently, LFN studies have also been extended to nontraditional materials and devices, such as nanowire transistors [115], [116], graphene devices [117], organic light-emitting diodes [118], and organic thin-film transistors [119], [120]. However, there are few reports of noise measurements on polymer solar cells. Bag et al. correlated the LFN of P3HT:PCBM BHJ solar cells with light-induced degradation [121]. Landi et al. investigated the dark LFN noise of P3HT:PCBM BHJ organic solar cells and correlated the noise spectrum with trapping states of the materials [122]. Nevertheless, the understanding of the relationship between LFN and carrier transport is still largely incomplete. The focus of the rest of this chapter will be on the measurement of $1 / \mathrm{f}$ noise in solar cells and photodiodes, and understanding how $1 / \mathrm{f}$ noise is related to the device physics. 


\subsection{Low Frequency Noise in Organic Solar Cells}

Organic solar cells are promising renewable energy sources that can achieve low production cost and extremely high production rates [15], [123]. Recent efforts have resulted in the highest power conversion efficiency of $10.7 \%$ reported by Mitsubishi Chemical [18], and the performance continues to improve. However, many challenges to understanding the underlying physics remain, such as the exciton loss mechanism [124], carrier recombination, and charge transport mechanisms [78], [125].

The poly (3-hexylthiophene-2,5-diyl) (P3HT) and [6,6]-phenyl C61-butyric acid methyl ester (PCBM) bulk heterojunction (BHJ) organic solar cell is a polymer-based organic photovoltaic device that has been extensively studied. It is well known that the thermal annealing conditions during the fabrication of P3HT:PCBM cells have a significant impact on the device characteristics, such as absorption spectra, series resistance [126], [127] and carrier mobilities [52]. Devices show better performance as the annealing temperature increases up to a certain level, usually between $110{ }^{\circ} \mathrm{C}$ and $160{ }^{\circ} \mathrm{C}$ depending on the fabrication conditions [127]. Studying the origin of such phenomenon will benefit the optimization of the fabrication organic solar cells. However, the traditional studies on the thermal annealing effect using current-voltage (IV) characteristics and atomic force microscopy (AFM) only reveal limited information related to carrier dynamics [127]. Additional methods can provide additional physical insights.

We study the dark LFN of P3HT:PCBM BHJ solar cells that are thermally treated at different annealing temperatures. The noise spectra of both the forward and reverse biased devices are measured and analyzed. In the forward bias, we show that the LFN level is proportional to the ratio of Hooge's parameter to carrier recombination lifetime, and LFN is higher for devices annealed at lower temperatures. Under reverse bias, the noise mechanisms are more complex since another noise generating process (i.e., tunneling current noise) becomes prevalent. We also demonstrate that the low-frequency noise is a material quality indicator related to the device performance.

The fabrication of P3HT:PCBM BHJ organic solar cells is described in Chapter 1. The active area of the devices was approximately $1 \mathrm{~mm}^{2}$. The active layer thickness was $85 \pm 5 \mathrm{~nm}$ as 
measured by a Veeco Dektak profilometer. The photovoltaic performance was characterized by a calibrated Oriel solar simulator, and the results are tabulated in Table 5.1. The dark currentvoltage (IV) characteristics were measured with a Keithley 2400 source meter.

Table 5.1: Open-circuit voltage $\left(V_{o c}\right)$, short-circuit current density $\left(J_{s c}\right)$, fill factor $(F F)$, and power conversion efficiency (PCE) of P3HT:PCBM BHJ solar cells annealed at $60^{\circ} \mathrm{C}, 95{ }^{\circ} \mathrm{C}$, $110^{\circ} \mathrm{C}$ and $140^{\circ} \mathrm{C}$.

\begin{tabular}{|c|c|c|c|c|}
\hline Temperature $\left({ }^{\circ} \mathrm{C}\right)$ & $\mathrm{V}_{\mathrm{oc}}(\mathrm{V})$ & $\mathrm{J}_{\mathrm{sc}}\left(\mathrm{mA} / \mathrm{cm}^{2}\right)$ & FF & PCE \\
\hline 60 & 0.56 & 6 & 0.39 & $1.3 \%$ \\
\hline 95 & 0.58 & 6 & 0.39 & $1.3 \%$ \\
\hline 110 & 0.60 & 8 & 0.45 & $2.2 \%$ \\
\hline 140 & 0.62 & 8 & 0.49 & $2.4 \%$ \\
\hline
\end{tabular}

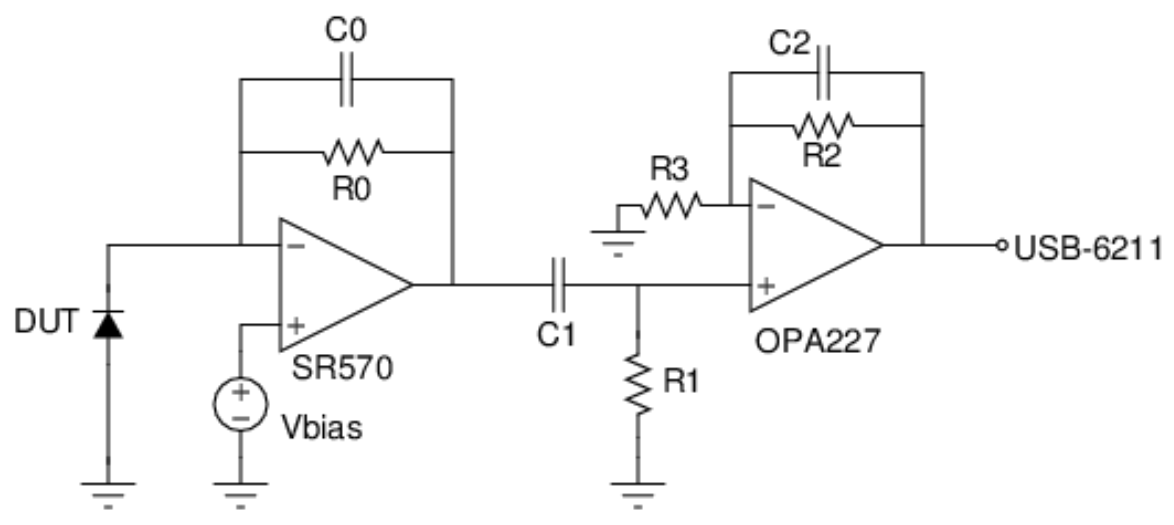

Figure 5.4: The circuit diagram for the low-frequency noise measurement. The bias on the devices under test is applied from the SR570 amplifier. C1 and R1 make a high-pass filter with $3 d B$ cut-off frequency of $0.3 \mathrm{~Hz}$. $C 2=1 \mathrm{nF}, R 2=10 \mathrm{k} \Omega$, and $R 3=1 \mathrm{k} \Omega$.

Figure 5.4 shows the experimental setup of the low-frequency noise measurement. The dark current from the organic solar cell was first amplified by an SR570 low-noise transimpedance amplifier (from Stanford Research Systems), which also provided the bias to the device under test. The device is placed in a metal case for measurement. The maximum bias voltage from the SR570 was $\pm 5 \mathrm{~V}$, which was sufficient for our application (-1V to $1 \mathrm{~V}$ depending on the dark current level). The output signal from the SR570 was passed through a high-pass filter, and then further amplified by a voltage amplifier built from a low-noise operational amplifier (OPA227 
from Texas Instruments). The SR570 amplifier was bandwidth limited below $10 \mathrm{kHz}$ or $2 \mathrm{kHz}$ depending on the gain. The $3 \mathrm{~dB}$ cut-off frequency of the high-pass filter was $0.3 \mathrm{~Hz}$. The output voltage of the SR570 was kept below $\pm 1 \mathrm{~V}$ to ensure that the amplifier operated in the linear region. For the second amplifier, $\mathrm{C} 2=1 \mathrm{nF}, \mathrm{R} 2=10 \mathrm{k} \Omega$, and $\mathrm{R} 3=1 \mathrm{k} \Omega$. The amplifier showed stable behavior during a frequency sweep test up to $20 \mathrm{kHz}$. Both amplifiers were powered by $\pm 12 \mathrm{~V}$ batteries for better noise immunity. The final output signal was digitized by a National Instruments USB-6211 data acquisition unit. The current noise power spectra were calculated by Fast Fourier Transform (FFT). To smooth the noise power spectra, 100 FFT traces were averaged based on Welch's method [128], [129] for each measurement. All of the measurements were done at room temperature. Although no systematic test on how the temperature influenced the LFN was done, two identical measurements carried out within a 10-hour interval showed very similar results.

Figure 5.5 shows the dark IV characteristics of four P3HT:PCBM organic solar cells annealed at different temperatures. Under low forward bias (below $0.5 \mathrm{~V}$ ), the IV characteristics of the 110 ${ }^{\circ} \mathrm{C}$ and $140{ }^{\circ} \mathrm{C}$ samples follow the diode equation

$$
I=I_{0}\left(e^{\frac{q V}{n T}}-1\right)
$$

where $\mathrm{I}_{0}$ is the reverse saturation current and $\mathrm{n}$ is the ideality factor. $\mathrm{I}_{0}$ and $\mathrm{n}$ can be obtained by fitting the curves in Figure 5.5 to Equation (5.8) between $3 \mathrm{kT} / \mathrm{q}$ and $0.5 \mathrm{~V}$. We find $\mathrm{I}_{0}$ and $\mathrm{n}$ to be $6.0 \times 10^{-8} \mathrm{~A} / \mathrm{cm}^{2}$ and 1.5 , respectively, at $110^{\circ} \mathrm{C}$, and $2.7 \times 10^{-8} \mathrm{~A} / \mathrm{cm}^{2}$ and 1.8 at $140{ }^{\circ} \mathrm{C}$. The IV characteristics of the devices annealed at $60{ }^{\circ} \mathrm{C}$ and $95{ }^{\circ} \mathrm{C}$ are symmetric in the low forward and reverse voltage range. This is an indicator of significant shunt leakage current, which is common in organic solar cells [130]. Since the IV characteristics deviate from Equation (5.8), $\mathrm{I}_{0}$ and $\mathrm{n}$ were not calculated for the two lower temperatures. 


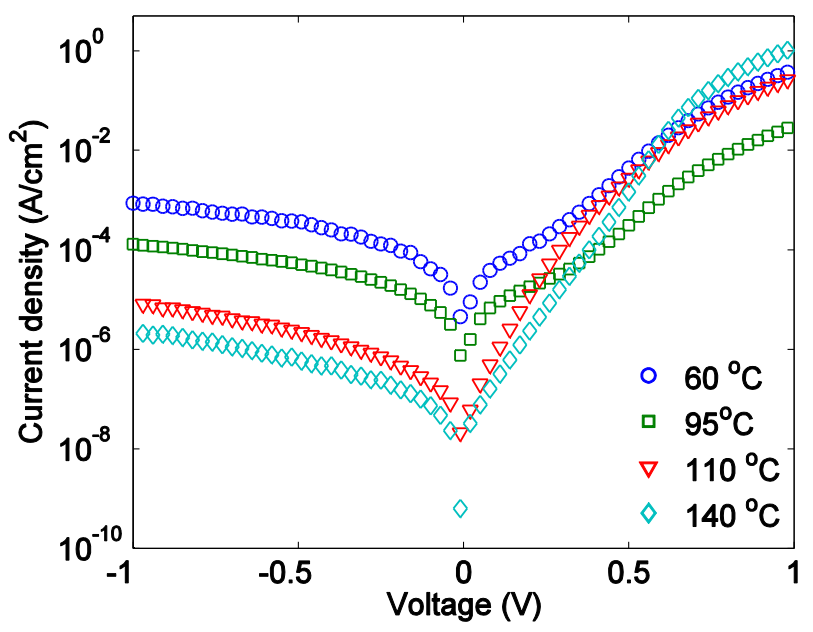

Figure 5.5: The dark current-voltage characteristics of four P3HT:PCBM devices annealed at $60^{\circ} \mathrm{C}, 95^{\circ} \mathrm{C}, 110^{\circ} \mathrm{C}$ and $140^{\circ} \mathrm{C}$.

Figure 5.6 shows four measured LFN spectrum examples for the four different annealing temperatures. All measured LFN curves are higher than the reference noise tested with a $1 \mathrm{M} \Omega$ resistor, which shows negligible LFN in the frequency range of interest. A general expression for the current noise power spectral density $(\mathrm{PSD}) \mathrm{S}_{\mathrm{I}}$ has the form

$$
S_{I} \propto \frac{I_{D C}^{\gamma}}{f^{\beta}}
$$

where $\mathrm{I}_{\mathrm{DC}}$ is the DC current level, $\beta$ is usually between 1 and 2 for " $1 / \mathrm{f}$ "-like spectra on different devices and operating conditions [112], [131], and $\gamma$ determines the relationship between $\mathrm{S}_{\mathrm{I}}$ and $\mathrm{I}_{\mathrm{DC}}$. 


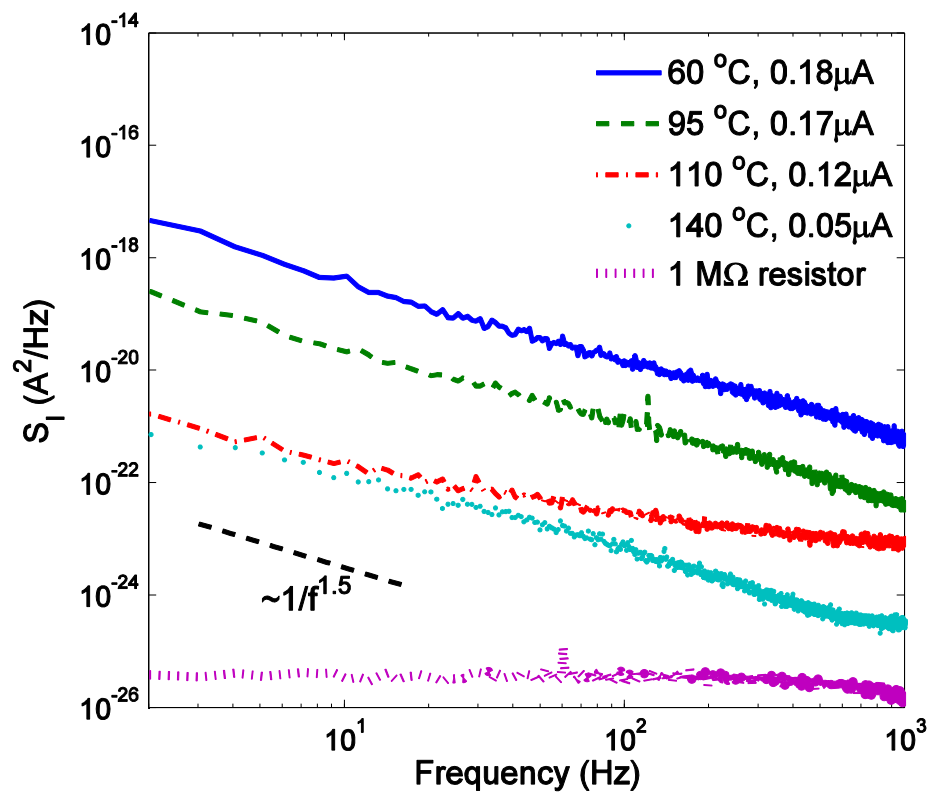

Figure 5.6: Four LFN spectrum examples under forward bias. The system noise is tested with an 1 M ohm resistor. The measured "1/f" slope is around 1.5 .

To compare the LFN of different devices, the current noise PSD at $10 \mathrm{~Hz}\left(\mathrm{~S}_{\mathrm{I} 0}\right)$ is plotted against $\mathrm{I}_{\mathrm{DC}}$ in Figure 5.7. The fitted $\gamma$ values are listed in Table 5.2. Two distinctive features are observed: for $60{ }^{\circ} \mathrm{C}$ and $95{ }^{\circ} \mathrm{C}$, the $\mathrm{S}_{\mathrm{I} 0} \sim \mathrm{I}_{\mathrm{DC}}$ relationship is almost linear ( $\gamma$ close to 1$)$ and $\mathrm{S}_{10}$ is relatively symmetric for the forward and reverse bias; for $110{ }^{\circ} \mathrm{C}$ and $140{ }^{\circ} \mathrm{C}$, however, $\gamma$ is close to 1 in forward bias and close to 2 in reverse bias, and $\mathrm{S}_{\mathrm{I} 0}$ is asymmetric for forward and reverse bias. The origin of this phenomenon can be explained in terms of the competition between generation-recombination (G-R) current noise and tunneling current noise, which will be elaborated later. 

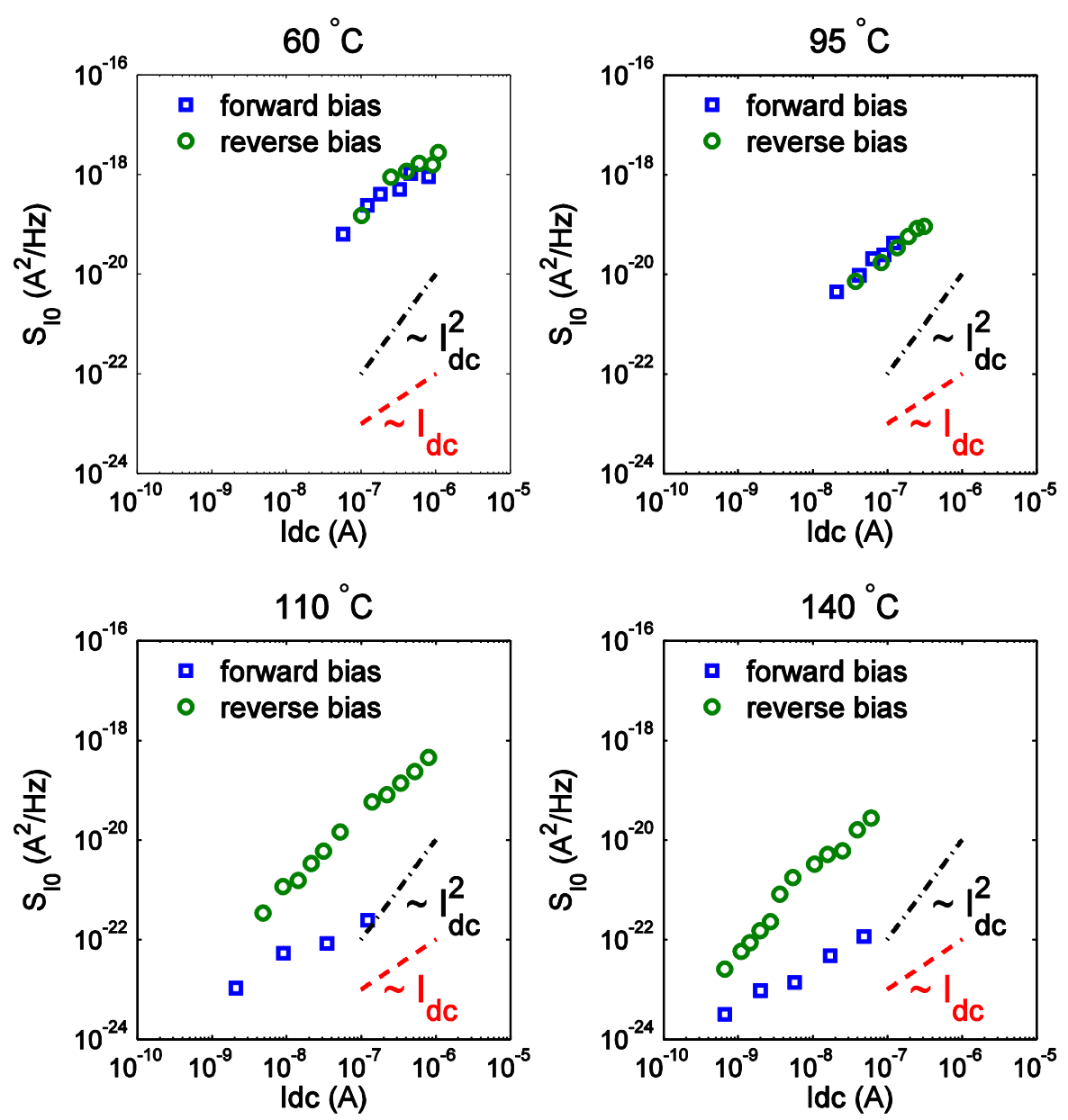

Figure 5.7: Current noise power spectral density at $10 \mathrm{~Hz}\left(S_{I 0}\right)$ versus forward and reverse DC dark currents of devices annealed at different temperatures.

\section{Table 5.2: The fitted $\gamma$ in Equation (5.9) for devices annealed at different temperatures.}

\begin{tabular}{|l|l|l|}
\hline Temperature $\left({ }^{\circ} \mathrm{C}\right)$ & $\gamma$, forward bias & $\gamma$, reverse bias \\
\hline 60 & 1.0 & 1.2 \\
\hline 95 & 1.2 & 1.2 \\
\hline 110 & 0.8 & 1.7 \\
\hline 140 & 0.8 & 1.7 \\
\hline
\end{tabular}


Although there is still some disagreement on the origin of the low-frequency noise (LFN) in electronic devices, mobility fluctuation [105]-[107] and number fluctuation [103], [104] are two of the leading theories that can explain significant subsets of the observations thus far. It is instructive to fit the LFN results of P3HT:PCBM BHJ solar cells to the theory of number fluctuation, which has as its origin from carrier trapping or surface recombination. The superposition of Lorentzian distributions in the frequency space as the result of carrier trapping can yield “1/f" -like noise spectra [114]. However, this will result in $\gamma \approx 2$ regardless of bias conditions [132], which contradicts our observations in the forward bias. The origin of 1/f noise has also been attributed to surface recombination [133]. However, the planar structure of BHJ solar cells does not have an exposed open surface area that can greatly influence the charge transport. It follows that number fluctuation does not apply to the BHJ devices structure studied in this work. Kleinpenning modeled the LFN generation in p-n junction diodes by extending Hooge's empirical law [106] to inhomogeneous media and derived formulas showing linear relationship between $S_{I}$ and $I_{D C}$ in the low voltage range [108], [109]. Here we use Kleinpenning's model to explain some aspects of the LFN results in Figure 5.7.

We choose Kleinpenning's formula for G-R limited current because the calculated ideality factors are closer to 2 than 1. In this scenario, the current noise PSD of P3HT:PCBM cells can be expressed as follows [108]:

$$
\mathrm{S}_{\mathrm{I}}=\frac{\alpha_{\mathrm{H}} \mathrm{cqI} \mathrm{I}_{\mathrm{DC}}}{\mathrm{f} \tau_{\mathrm{r}}}
$$

where $\alpha_{H}$ is the Hooge parameter, $I_{D C}$ is the DC dark current in Equation (1), $\tau_{\mathrm{r}}$ is the carrier recombination lifetime, and $c=2 / 3$ when the current is dominated by G-R [109]. Equation ( 5.10) can explain the $60{ }^{\circ} \mathrm{C}$ and $95{ }^{\circ} \mathrm{C}$ data in Figure 4 very well: $\gamma \approx 1$ and $\mathrm{S}_{\mathrm{I} 0}$ is symmetric in forward and reverse bias. However, for the $110{ }^{\circ} \mathrm{C}$ and $145^{\circ} \mathrm{C}$ data $\mathrm{S}_{\mathrm{I} 0}$ is unambiguously larger in reverse bias, and scales quadratically with $\mathrm{I}_{\mathrm{DC}}$. This suggests that there is another noise source that dominates in reverse bias but not for forward bias. The asymmetry in the noise characteristics was also observed in other material systems such as quantum well photodetectors [134]. 
Although the reverse current of P3HT:PCBM organic solar cells could increase rapidly beyond a specific electric field value (larger than $5 \times 10^{7} \mathrm{~V} / \mathrm{m}$ according to Ref. [38]), there is still no report on associating such current breakdown to carrier avalanche. Instead, in Ref. [38], it was reported that tunneling current can be the major effect in reverse bias. Therefore, we believe impact ionization may not play a major role in the reverse bias for P3HT:PCBM organic solar cells.

We attribute the excess LFN in the reverse bias from devices annealed at $110{ }^{\circ} \mathrm{C}$ and $140{ }^{\circ} \mathrm{C}$ to the tunneling current noise. While the total dark current in diodes may consist of diffusion, generation-recombination, shunt leakage, and tunneling, only the tunneling current, which increases with the electric field [23], [135], is significantly larger in the reverse bias than forward bias. The thin active layers of the P3HT:PCBM devices favor a tunneling mechanism. In fact, trap-assisted tunneling [136], [137] is probable since organic solar cells are known to have high densities of localized states. Previous work has shown that the tunneling current noise PSD generally has $\gamma \approx 2$ for planar devices [138]-[140]. When the device is under forward bias, the current noise arises primarily from the recombination current so that $\gamma \approx 1$. However, the tunneling current noise dominates the recombination current noise in the reverse bias for the 110 ${ }^{\circ} \mathrm{C}$ and $145{ }^{\circ} \mathrm{C}$ devices, which drives $\gamma$ close to 2 . At the same time, $\gamma \approx 1$ in the reverse bias for the $60{ }^{\circ} \mathrm{C}$ and $95{ }^{\circ} \mathrm{C}$ data indicates that the magnitude of the tunneling current noise may still be less than the recombination current noise owing to the higher degree of material disorder which facilitates recombination in the active layer.

However, the difference in the tunneling current noise is less obvious among the four different temperatures. From Figure 5.7, the magnitude of LFN in the reverse bias for the $60{ }^{\circ} \mathrm{C}$ and $95{ }^{\circ} \mathrm{C}$ devices where the tunneling current noise is not prevalent is no larger than the $110{ }^{\circ} \mathrm{C}$ and $145{ }^{\circ} \mathrm{C}$ devices where the tunneling current noise dominates. It follows that there is at least no significant reduction in the tunneling current noise when the annealing temperature increases. While the improved morphology as a result of higher annealing temperature should yield less pronounced trap-assisted tunneling, in fact, the tunneling current is also related to other factors such as the material band structure and the carrier effective mass [23], [135]. While there have been few studies on how the carrier effective mass changes with the annealing temperature, it is observed that thermal annealing modifies the band structure of P3HT:PCBM films manifested by 
the red-shift in the absorption spectrum [126]. Therefore, higher annealing temperature does not necessarily result in lower tunneling current noise. Future work is required to understand the physical details of the relationship between the tunneling current noise and thermal annealing.

It is widely acknowledged that the thermal annealing of BHJ organic solar cells improves the crystallinity of the active layer, which further influences the absorption spectrum, series resistance [126], [127] and charge transport properties such as the charge carrier mobilities [52] and lifetimes [141]. While the traditional methods such as current-voltage (IV) characteristics and atomic force microscopy (AFM) only provide qualitative and mingled insights [127], the LFN characteristics provide a clear indicator of carrier transport properties. The current noise PSD in the forward bias can be related to the ratio of the Hooge parameter $\alpha_{H}$ to the carrier recombination lifetime $\tau_{\mathrm{r}}$ as shown in Equation (5.10). It is known that the value of $\alpha_{\mathrm{H}}$ varies with type of material, and a low-value $\alpha_{\mathrm{H}}$ usually indicates better material quality and higher device reliability [112], [142]. Thermal treatment of BHJ devices usually results in increased $\tau_{\mathrm{r}}$ [141]. Figure 5.8 plots the $\alpha_{H} / \tau_{\mathrm{r}}$ ratio versus annealing temperature. Note that $\alpha_{H} / \tau_{\mathrm{r}}$ is three orders of magnitude less for the $140{ }^{\circ} \mathrm{C}$-annealed device compared to the device annealed at 60 ${ }^{\circ} \mathrm{C}$. This is consistent with the observation that P3HT:PCBM devices thermally treated beyond the glass transition point have better photovoltaic performance compared to those annealed at low temperatures as shown both in Table 5.1 and literature [126]. There is still no report on whether $\alpha_{H}$ is also a function of the annealing temperature for P3HT:PCBM BHJ solar cells. To extract the carrier lifetimes from the LFN results, the quantitative relationship between the Hooge's parameter and annealing temperatures could be the subject of future work. 


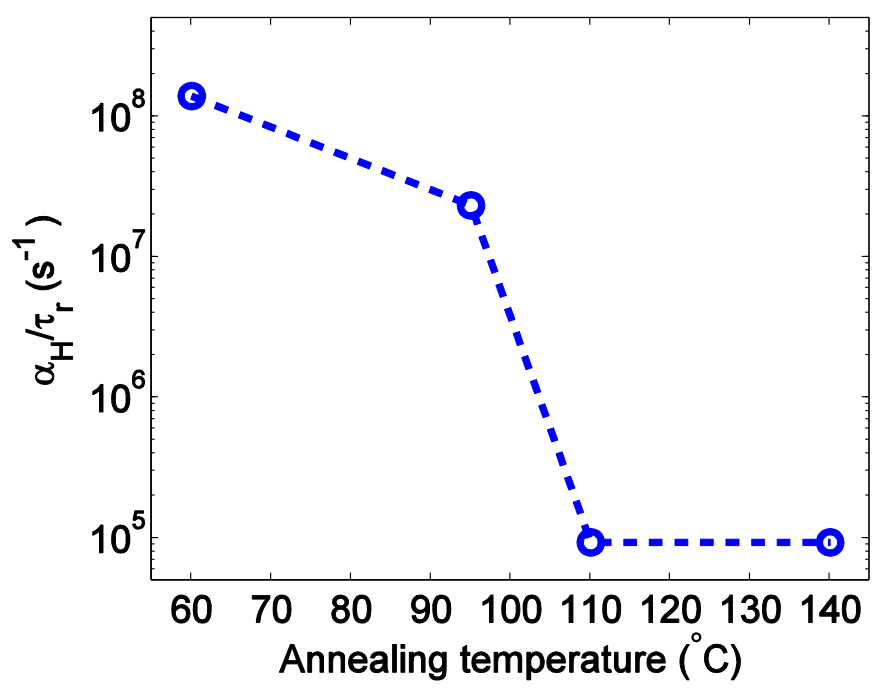

Figure 5.8: The ratio of the Hooge parameter to the carrier recombination lifetime as a function of the annealing temperatures. All values are calculated at $I_{D C}=1 \times 10^{-7} \mathrm{~A}$ in the forward bias.

The LFN measurement has the potential of being applied in organic solar cell manufacturing and device optimization. Although the power conversion efficiency is the ultimate benchmark of solar cells, it lacks the details that are beneficial for optimization of device performance. The underlying physical parameters such as carrier lifetimes provide the guidelines for device optimization. Compared with other methods used to measure carrier lifetimes such as transient photoconductivity method [46], [78], [143], the transient photovoltage method [144], [145], impedance spectroscopy [146] and time-resolved vibrational spectroscopy [147], the LFN measurement has the advantage of being compact and not requiring sophisticated test equipment in the experimental setup. It can potentially be integrated as part of a more comprehensive testing system.

\subsection{Low-Frequency Noise in MUTC Photodiodes}

As introduced in Chapter 2, the modified uni-traveling carrier (MUTC) photodiodes are highspeed and high-power inorganic photodiodes that are widely used in various analog optical links [29]-[31]. Recently, MUTC photodiodes have become crucial components in ultrastable 
microwave generators whose phase noise is significantly lower than their electrical counterparts [7]. The difference between various flavors of MUTC photodiodes [32], [148], which is usually label as "MUTCx" where $\mathrm{x}$ is a numeric number starting at 1 , is essentially the way to manage space charge to achieve different design goals, such as high speed or high power. In practice, tweaking the thickness of epitaxial layers and doping levels achieve space charge management. For the interest of this dissertation, MUTC4 photodiodes that are optimized to operate at $10 \mathrm{GHz}$ are investigated.

Similar to the organic solar cells, the low-frequency noise (LFN) in MUTC4 photodiodes is closely related to their physical properties. LFN measurements in the dark condition have been carried out on MUTC4 photodiodes with mesa diameters of $50 \mu \mathrm{m}, 40 \mu \mathrm{m}$ and $34 \mu \mathrm{m}$. The measurement circuit is very similar to Figure 5.4. The voltage bias on the photodiodes is provided by an SR570 pre-amplifier, and a high-pass filter with $0.3 \mathrm{~Hz} 3 \mathrm{~dB}$ bandwidth removes the DC offset from the SR570. The gain of the post-amplifier (the second amplifier in Figure 5.4) is either 11 or 50 to boost the voltage output from the pre-amplifier above the noise floor of the USB-6211. The sampling rate of the data acquisition unit USB-6211 is set to $50 \mathrm{kHz}$ to avoid aliasing in sampling. The total sampling duration for each measurement is $10 \mathrm{~s}$, and the noise spectrum is obtained with the same FFT routine used in the previous section.
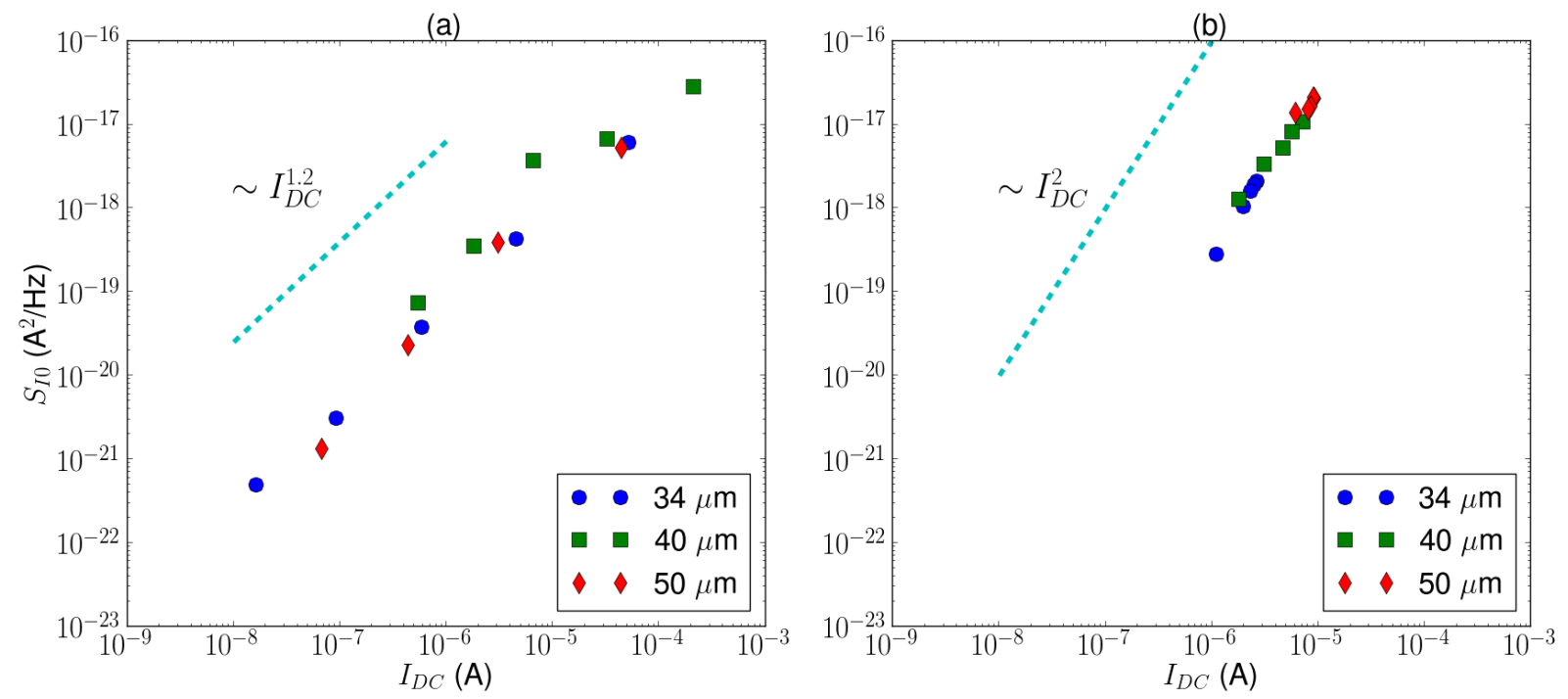

Figure 5.9: The dark low-frequency current noise power spectral density at $10 \mathrm{~Hz}$ of MUTC4 photodiodes with different active area sizes in the (a) forward and (b) reverse bias conditions. 
The dashed-lines indicate the fitted $\gamma$ value, the power law relationship between the current noise power and the DC dark current level.

The relationship between the dark low-frequency current noise power spectral density (PSD) and DC current level of MUTC4 photodiodes is shown in Figure 5.9 (a) (forward bias) and Figure 5.9 (b) (reverse bias). It can be seen that the diameter of the active area does not significantly influence the noise level for both forward and reverse bias, an indication that LFN is a bulk effect rather than a surface effect. Similar to organic solar cells, the LFN PSD can be related to the DC current using Equation ( 5.9). The fitted value of $\gamma$ in Equation ( 5.9) is close to $1(\gamma \sim 1.2)$ in the forward bias condition and approximately 2 in the reverse bias condition. The asymmetry of the noise characteristics in the forward and reverse conditions suggests there is a noise generating process that is prevalent in reverse bias but suppressed in forward bias. Among possible noise generation sources such as diffusion, generation-recombination, shunt leakage, tunneling, and impact ionization, only the tunneling current noise is much larger in reverse bias than forward bias. In fact, the high electrical field in the MUTC4 structure is very likely to make the tunneling mechanism pronounced, which will be discussed later in this section.

To understand the noise characteristics of MUTC4 photodiodes under illumination, another measurement setup as shown in Figure 5.10 was assembled. Different from the single-diode configuration shown in Figure 5.4, balanced photodetection is employed to minimize the undesirable interference of the common-mode laser noise. Two photodiodes with similar physical properties are arranged in series, and they are reverse biased using a pair of positive and negative voltage sources. The middle point between the photodiodes is the virtual ground, and can be sensed by a low-noise amplifier. A continuous-wave (CW) fiber laser with the emission wavelength of $1550 \mathrm{~nm}$ is first branched by a $3 \mathrm{~dB}$ optical splitter, and then coupled into the two photodiodes after attenuation. The bias sources, two Keithley 2400 sourcemeter units, also measure the photocurrent levels on the photodiodes respectively. The optical power coupled into the photodiodes is adjusted to match the photocurrent in each photodiode. If the photodiodes are well balanced, only the differential signal from the photodiodes is measured and the commonmode signals, such as the photonoise induced by the relative intensity noise (RIN) of the CW laser and DC photocurrent offset, are rejected. At the same time, there is also uncorrelated photonoise associated with the photodiodes themselves. Since this type of noise is uncorrelated on two separate photodiodes, the difference is not canceled. It should appear as a zero-mean 
random current noise that is sensed by the low-noise preamplifier SR570. The root mean square of the final photonoise should be the sum of root mean square photonoise from the two photodiodes. The voltage output of SR570 is digitized by a National Instrument data acquisition unit USB-6211. The noise spectrum is obtained by running the same FFT routine used in the dark LFN measurement. Compared with the measurement setup in Figure 5.4, this balanced detection scheme not only minimizes the influence of RIN, but also the large common DC offset in each photodiode, which can overload the amplifier very easily and prevent the amplifier from achieving the required gain in the non-balanced detection configuration.

A side note about the balanced photodetection setup shown in Figure 5.10 is that it cannot cancel the shot noise from the laser. The quantum nature of the shot noise dictates that the number of photons arriving at two photodiodes is subjected to number fluctuation, even though these photons are from the same laser and synchronized in time when they leave the laser. That is why an ultrastable laser is needed to accurately determine the phase noise floor of a photodiode [149]. However, other types of laser noise such as flicker noise or power fluctuation, may have different origins from shot noise, and thus may be effectively canceled by balanced photodetection. Here we are most interested in the low-frequency part of the spectrum above the white noise floor, and balanced photodetection is still a very viable method for this purpose.

Before measuring MUTC4 photodiodes, a calibration measurement was carried out on two similar packaged commercial photodiodes with responsivities of $0.95 \mathrm{~A} / \mathrm{W}$ and $0.91 \mathrm{~A} / \mathrm{W}$ and bandwidth of $1 \mathrm{GHz}$ from Applied Optoelectronics Inc. (part number PD1000-FA-10-H-B) to ensure that the system worked as expected. The dark current was only a few nA under $-5 \mathrm{~V}$ reverse bias. The two packaged single photodiodes were configured as balanced photodiodes according to Figure 5.10 for the photonoise measurement. 


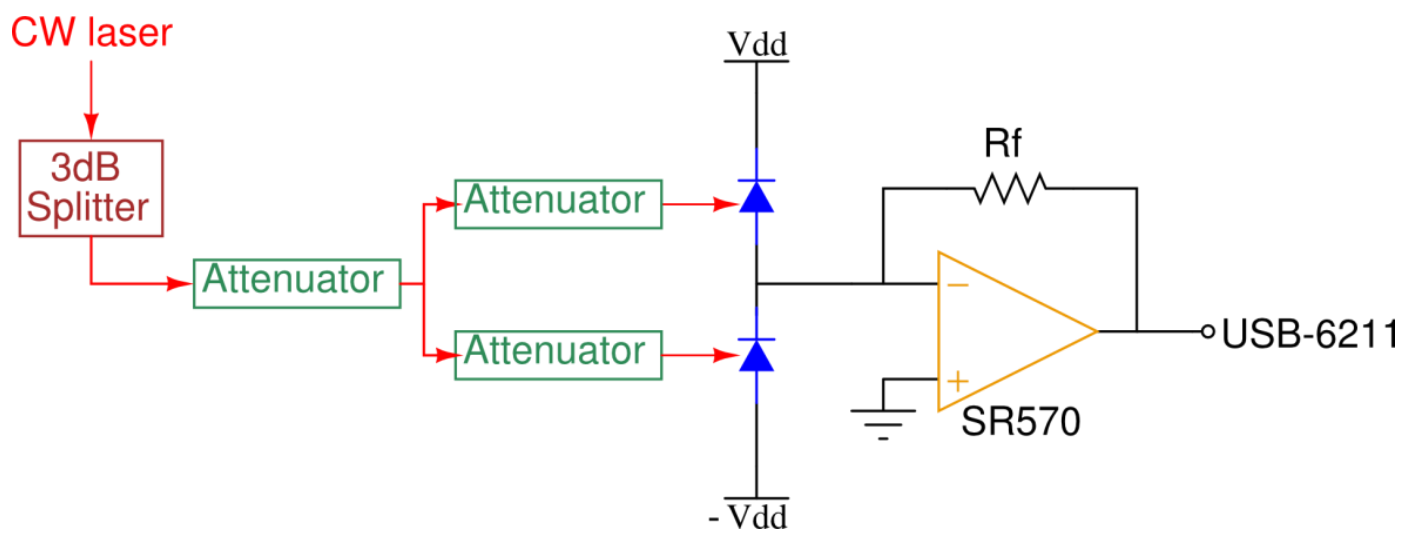

Figure 5.10: Photonoise measurement with balanced photodiodes.

(a)

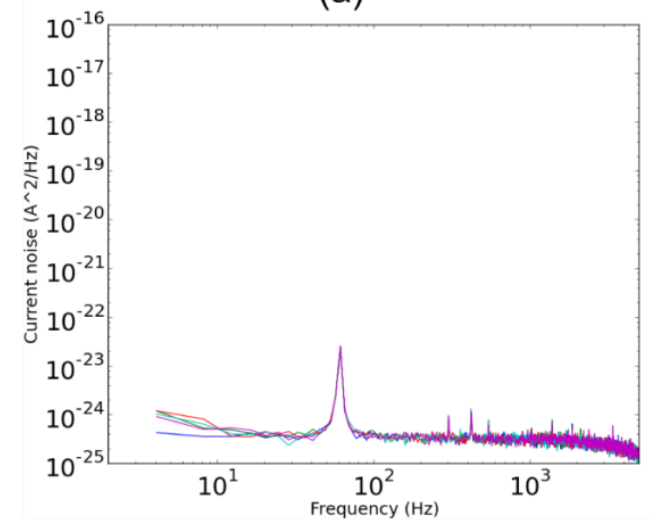

(b)

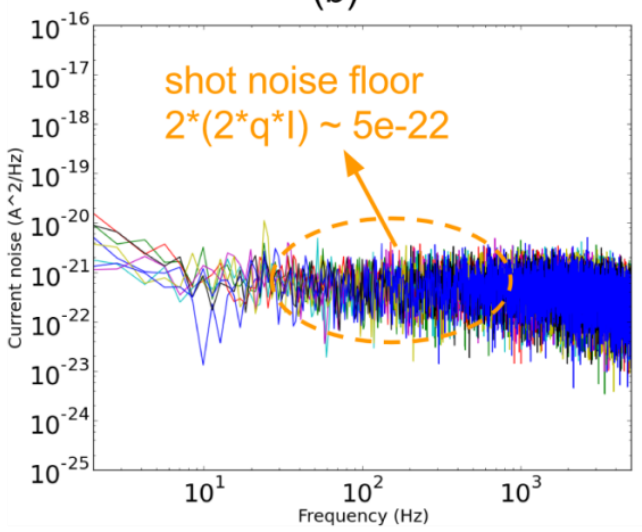

Figure 5.11: Multiple repetitive measurements of (a) dark noise and (b) photonoise (DC photocurrent $\sim 1 \mathrm{~mA}$ ) of commercial photodiodes from AOI under $-5 \mathrm{~V}$ reverse bias.

Figure 5.11(a) and Figure 5.11 (b) show the dark noise and photonoise, respectively, of AOI commercial photodiodes under $-5 \mathrm{~V}$ reverse bias. Measurements under identical conditions were repeated several times to ensure the consistency of the results. Both shot noise and thermal noise should contribute to the overall measured noise. The thermal current noise from a device with $100 \mathrm{M} \Omega$ impedance, which is the lower bound for the impedance of AOI photodiodes under $-5 \mathrm{~V}$ bias, is approximately $1.6 \times 10^{-28} \mathrm{~A}^{2} / \mathrm{Hz}$ according to Equation ( 5.3). The shot noise level corresponding to the $5 \mathrm{nA}$ DC current is $1.6 \times 10^{-27} \mathrm{~A}^{2} / \mathrm{Hz}$. Owing to the low dark current level, the noise in Figure 5.11 (a) is determined by the amplifier noise floor, which is much larger than both thermal noise and shot noise. The noise peak at $60 \mathrm{~Hz}$ is primarily due to interference coupled from the power lines. The photonoise of the same diodes with photocurrent level of 
$1 \mathrm{~mA}$ and reverse bias of $-5 \mathrm{~V}$ is plotted in Figure 5.11 (b). We can see that the shot noise, calculated to be $5 \times 10^{-22} \mathrm{~A}^{2} / \mathrm{Hz}$, after multiplication by a factor of 2 because there are noise contributions from the two uncorrelated noise sources, is close to the measured noise level, which is also well above the amplifier noise floor in Figure 5.11 (a). Although there are " $1 / \mathrm{f}$ "like trends in the noise spectra in both Figure 5.11 (a) and Figure 5.11 (b), they are too weak to be correctly quantified at this point.

The photonoise associated with different photocurrent levels was also measured, and the results are shown in Figure 5.12. The noise spectra do not show significant dependence on the bias for the same photocurrent level, an indication that the noise associated with the dark charge does not play a big role for this type of photodiode. Also, in Figure 5.12 (c) the " $1 / \mathrm{f}$ " signature in the low-frequency region does not become obvious until the photocurrent is high. All of above indicates that our measurement system functions correctly.

We proceeded to measure the photonoise of MUTC4 photodiodes with the balanced detection setup in Figure 5.10. The responsivities of the photodiodes under test are $0.14 \mathrm{~A} / \mathrm{W}$ and $0.2 \mathrm{~A} / \mathrm{W}$, respectively. The photodiodes were packaged by a third-party company (Finisar); Figure 5.13 is a picture of one of the devices. The photonoise for different photocurrent levels up to $1 \mathrm{~mA}$ was measured under $0 \mathrm{~V}$ and $-4 \mathrm{~V}$ bias. Different from the AOI commercial photodiodes, the " $1 / \mathrm{f}$ " features in the photonoise spectra of MUTC4 photodiodes are conspicuous for all conditions. More interestingly, for zero bias the photonoise increases with photocurrent as expected, but the trend is reversed for $-4 \mathrm{~V}$ bias. The relationship between the current noise PSD (at $10 \mathrm{~Hz}$ ) and the photocurrent level for zero bias and $-4 \mathrm{~V}$ bias is plotted in Figure 5.15. Not surprisingly, while the value of $\gamma$ is still between 1 and 2 for the zero bias condition, it becomes negative when the devices are under $-4 \mathrm{~V}$ reverse bias. Although the abnormal trend associated with the $-4 \mathrm{~V}$ bias is puzzling, it is consistent with the previous conclusion that the tunneling current mechanism is the dominant noise source in reverse biased MUTC4 photodiodes, which is reflected by the asymmetry of noise characteristics in Figure 5.9. 
(a)

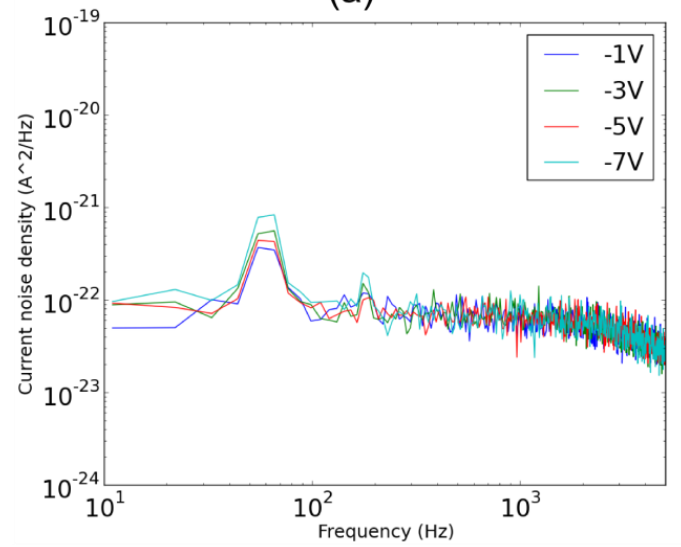

(b)

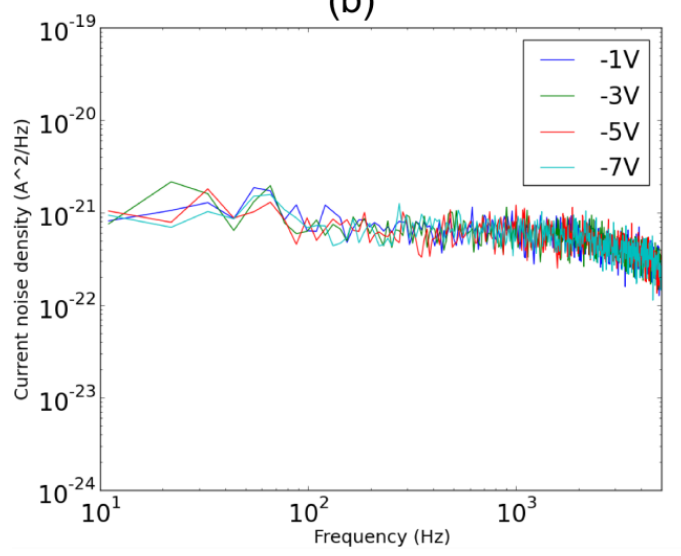

(c)

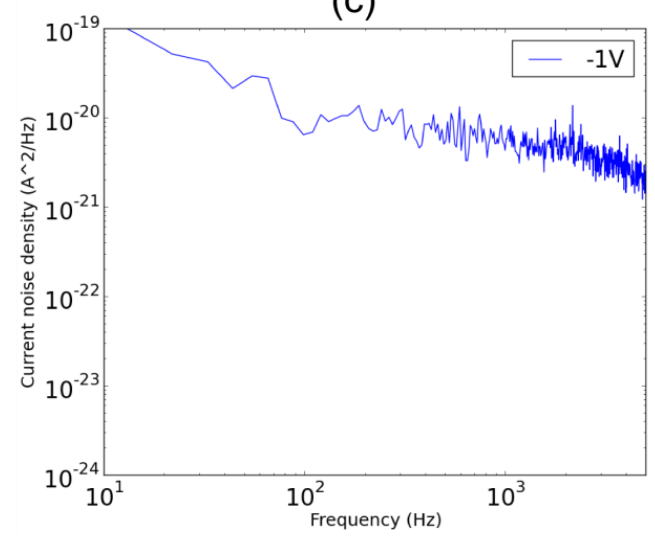

Figure 5.12: The photonoise of AOI commercial photodiodes with DC photocurrent levels of (a) $0.1 \mathrm{~mA}$, (b) $1 \mathrm{~mA}$ and (c) $7 \mathrm{~mA}$ under reverse bias of $-1 \mathrm{~V},-3 \mathrm{~V},-5 \mathrm{~V}$ and $-7 \mathrm{~V}$. For (c), only data under reverse bias of $-1 V$ is measured because of the unstable current under higher reverse bias. Also, the noise floor begins rolling off above $1 \mathrm{kHz}$ because of the bandwidth limit of the amplifier. 


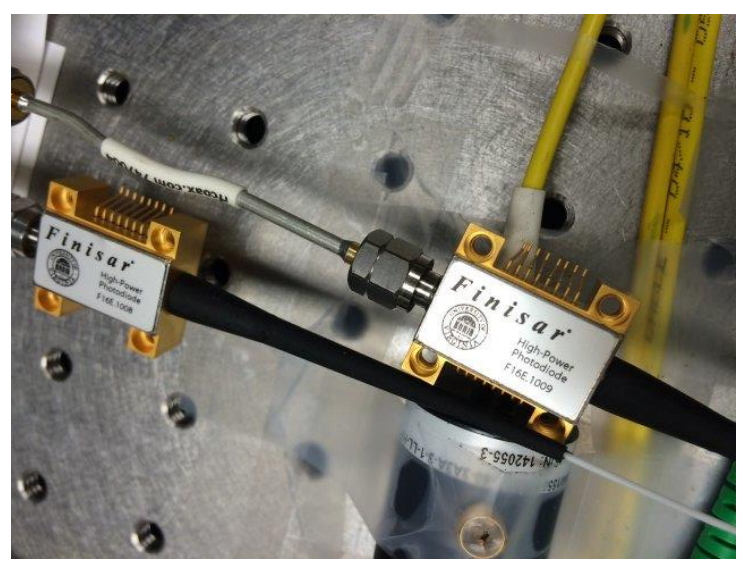

Figure 5.13: Packaged MUTC4 photodiodes from Finisar.

(a)

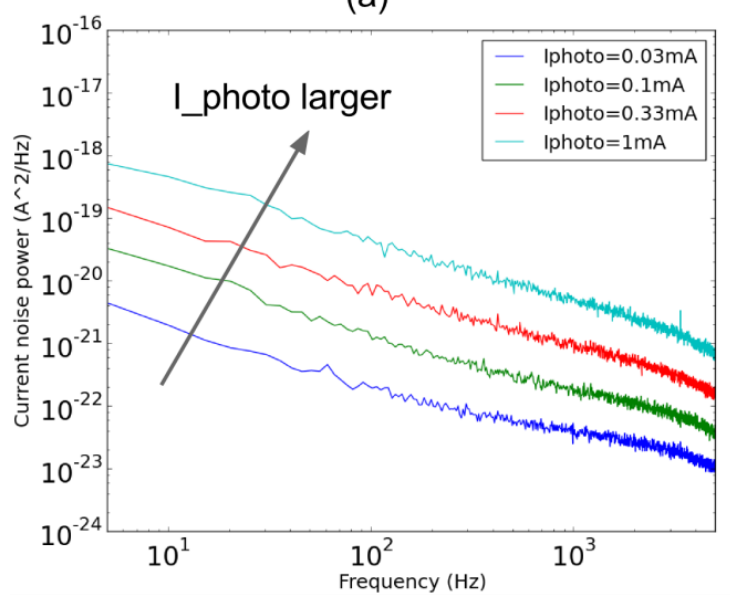

(b)

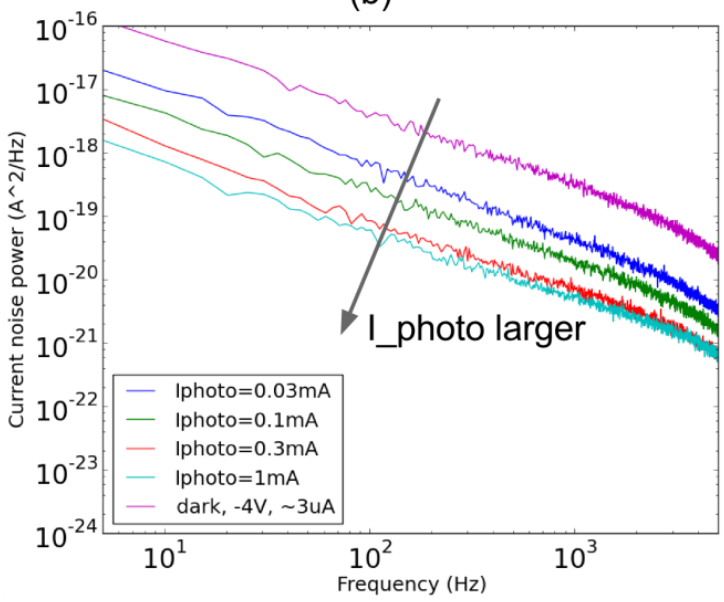

Figure 5.14: The photo-LFN of packaged MUTC4 photodiodes under (a) OV and (b) -4V bias. 


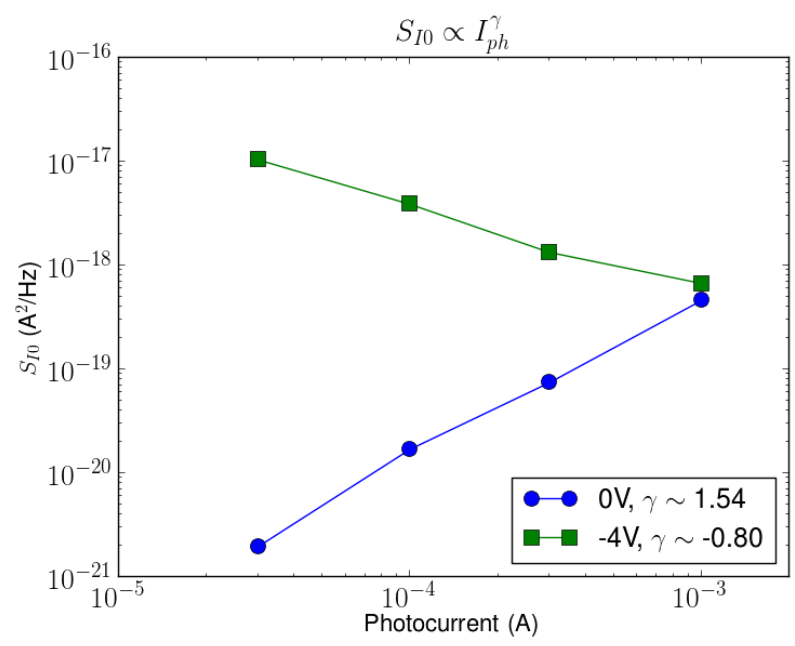

Figure 5.15: Photonoise PSD at $10 \mathrm{~Hz}$ versus averaged photocurrent level for MUTC4 photodiodes.

It is instructive to understand the tunneling current by analyzing the carrier dynamics in a typical p-n junction diode. The tunneling process is a quantum-mechanical effect, and can be understood as the direct charge conduction through a potential barrier, which can happen even if the kinetic energy of the charge is smaller than the potential barrier height. In the context of semiconductor diodes, there are several factors, such as the barrier height (usually equal to the band gap for a homojunction diode), the carrier effective mass, and the availability of energy states in the tunneling source and destination [23]. When a highly-doped p-n junction is in equilibrium (zero bias, no illumination) as shown in Figure 5.16(a), there is no tunneling because electrons in one side do not have corresponding vacant receiving states on the other side. When the diode is reverse biased, two types of tunneling, band-to-band tunneling and trap-assisted tunneling, are responsible for the tunneling related dark charge conduction. As shown in Figure 5.16(b), for the case of band-to-band tunneling, the electrons in the valence band of the p-type material can directly tunnel to the unoccupied states in the conduction band of the n-type material through a barrier with height of $E_{g}$. If there are trap states in the band gap as shown in Figure 5.16(c), the p-side electrons in the valence band can also first tunnel to the trap site, and then to the n-side vacancies in the conduction band, which is called the trap-assisted tunneling. It is easy to see that the trap-assisted tunneling is also related to the trap energy level and densities [139]. 
The tunneling probability $T_{t}$ derived from the triangle barrier model, which is a good approximation for semiconductor diodes in the case of band-to-band tunneling, can be written as [23]

$$
\mathrm{T}_{t} \approx \exp \left(-\frac{8 \pi \sqrt{2 m^{*}} E_{g}^{1.5}}{3 q h F}\right)
$$

where $\mathrm{m}^{*}$ is the carrier effective mass, $\mathrm{E}_{\mathrm{g}}$ is the band gap, $\mathrm{h}$ is Planck's constant, and $\mathrm{F}$ is the electric field. From Equation ( 5.11), we can see that a larger electric field results in higher tunneling probability and thus larger current, if other physical parameters remain the same.

Figure 5.17 is a plot of the simulated electrical field in a typical MUTC4 photodiode. It shows that the high electric field near the junction decreases as the optical power increases owing to the space-charge effect. It is tempting to argue that the reduction of the LFN as the photocurrent increases in the case of $-4 \mathrm{~V}$ bias results from the decreasing electric field. However, we only measure the photocurrent up to $1 \mathrm{~mA}$, which corresponds to current density of $0.08 \mathrm{kA} / \mathrm{cm}^{2}$ for a typical $40-\mu \mathrm{m}$ diameter MUTC4 photodiode. This is not high enough to induce any significant change in the electric field as can be seen in Figure 5.17. Therefore, the abnormal trend at $-4 \mathrm{~V}$ bias in Figure 5.15 is probably not caused by band-to-band tunneling. On the other hand, it is possible that the photogenerated carriers partially fill the trap states in the band gap, suppressing trap-assisted tunneling. This effect would be reflected in Figure 5.14 with reduced LFN PSD as the photogeneration increases. Among other noise generation mechanisms (shunt leakage, diffusion, generation-recombination, band-to-band tunneling), only trap-assisted tunneling can explain the anomaly at $-4 \mathrm{~V}$ bias in Figure 5.14 and Figure 5.15. However, further investigation is needed to reveal the related physical details. 
(a)

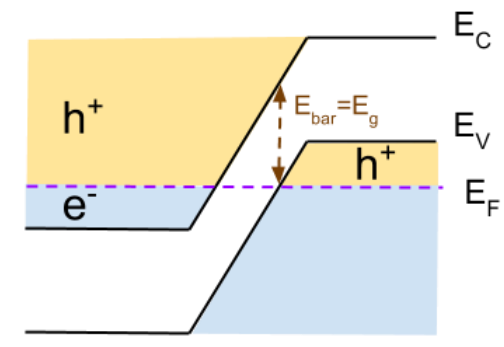

(b)

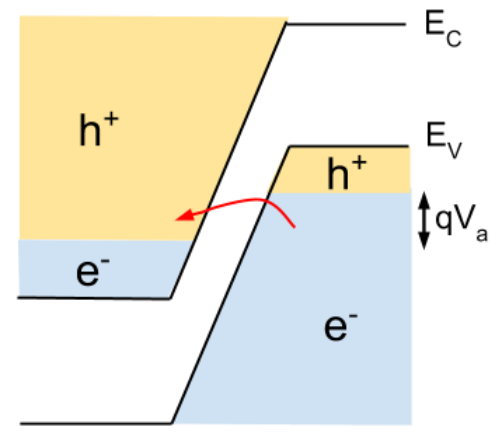

(c)

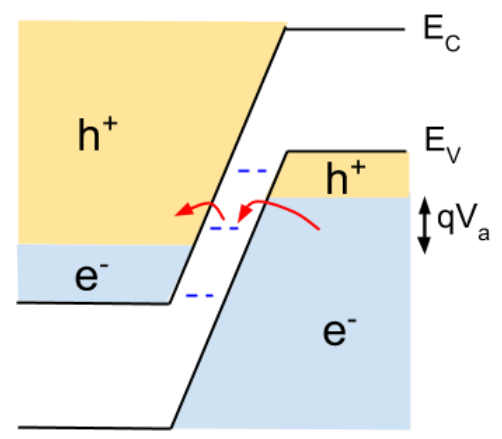

Figure 5.16: The illustration of (a) a highly-doped p-n junction (b) band-to-band tunneling and (c) trap-assisted tunneling. The tunneling barrier height $E_{b a r}$ usually equals the band gap $E_{g}$ for a homojunction diode.

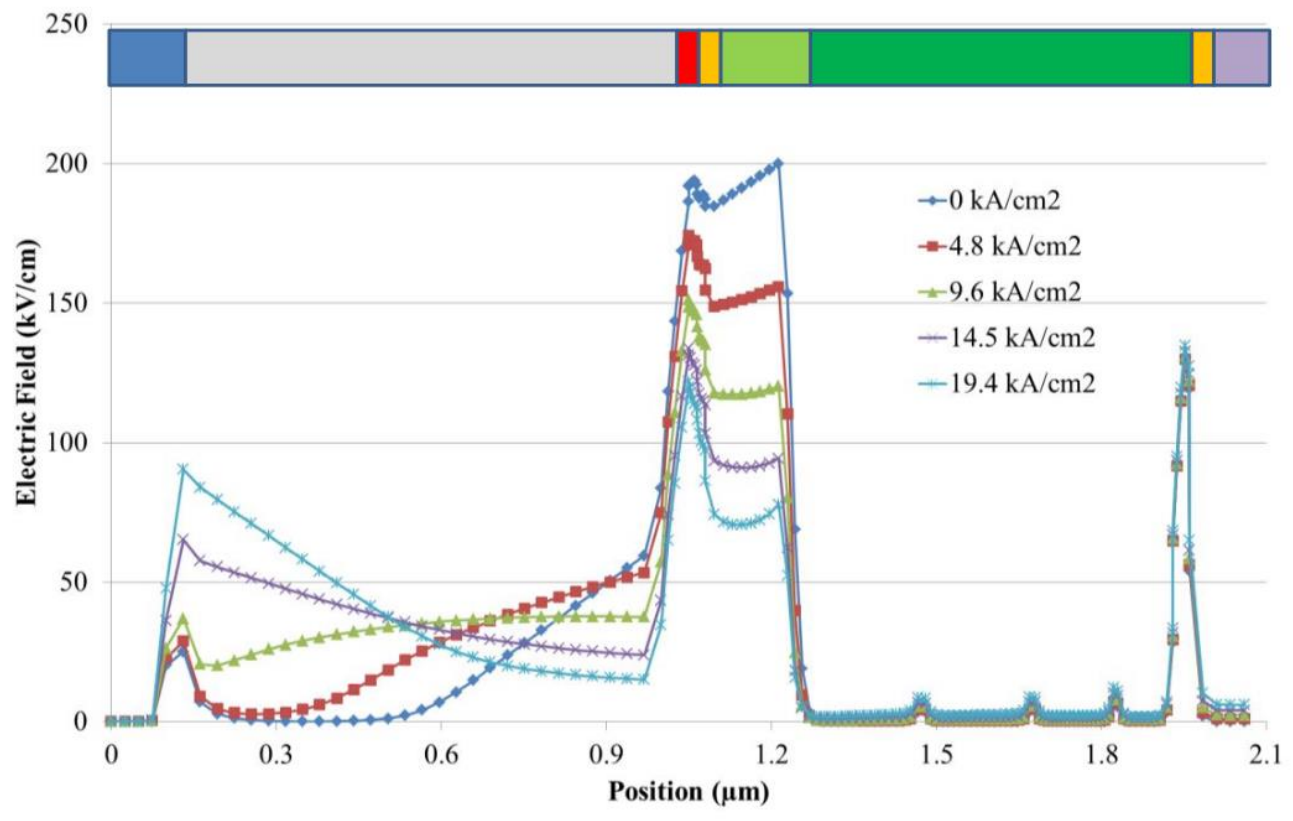

Figure 5.17: The electric field profiles of a typical MUTC4 photodiode under different illumination levels. Note that for a $40 \mu \mathrm{m}$-diameter MUTC4 photodiode, the $4.8 \mathrm{kA} / \mathrm{cm} 2$ current density corresponds to $60 \mathrm{~mA}$ photocurrent. This figure is from Ref[33]. 


\subsection{Up-conversion of Low-Frequency Noise}

In Sections 5.2 and 5.3 of this chapter, it has been demonstrated that the low frequency noise near DC is a good indicator of the physical properties of optoelectronic devices. However, it is also important to understand how this type of noise may affect the device performance in some applications. MUTC4 photodiodes are frequently deployed in high-power and high-speed applications, such as analog optical links and microwave signal generations. Recently, Dr. Diddams' group at National Institute of Standards and Technology (NIST) demonstrated microwave generation with record low phase noise near $10 \mathrm{GHz}$ using UVA high-power photodiodes [149]. While the thermal noise, shot noise and AM-PM noise contributions to phase noise have been studied in detail, the extent to which the low frequency noise, or $1 / \mathrm{f}$ noise near $\mathrm{DC}$, affects the noise performance near the RF carrier frequency is not well understood. This section tries to establish a basic framework for $1 / \mathrm{f}$ noise up-conversion.

The up-conversion of $1 / \mathrm{f}$ noise near $\mathrm{DC}$ to high frequency is a commonly observed phenomenon in semiconductor materials and devices [150], [151]. It has been actively studied for amplifiers based on devices such as BJT [152] and HBT [153]. Usually, the noise generation in a system with an input and an output is modeled by the fluctuation of its "transfer function", which closely reflects the system properties. For example, the dark 1/f noise generation near DC in a semiconductor material is modeled by its "flickering" conductance or resistance [109]. In a BJT amplifier with common-emitter configuration, the $1 / \mathrm{f}$ noise near DC is modeled by the "flickering" gain of the amplifier, which is related to the other parameters such as the collectorcurrent-dependent effective emitter resistance [152]. Since the "transfer function" of a photodiode is its responsivity, we can model the noise generation of a photodiode with a fluctuating responsivity. This proposition lays the foundation of the $1 / \mathrm{f}$ noise up-conversion in photodiodes.

Figure 5.18 shows the photocurrent and the mean of responsivity as a function of the input optical power in a typical photodiode. In the small-signal condition when the input optical power 
is small (e.g., operating point $\mathrm{P}_{\mathrm{a}}$ in Figure 5.18), the mean of responsivity is independent of the input power. Therefore, the "flickering" responsivity can be expressed as

$$
R=R_{0}\left[1+\sum_{n} X_{n}(t)\right]=R_{0}\left[1+X_{1}(t)+X_{2}(t)+\cdots\right]
$$

where $\mathrm{R}_{0}$ is the small-signal mean of responsivity, and $\mathrm{X}_{\mathrm{n}}(\mathrm{t})$ (with $\mathrm{k}=0,1,2, \ldots$ ) manifests different random processes modeling the generation of $1 / \mathrm{f}$ noise. As we have seen earlier in this chapter, there may be more than one $1 / \mathrm{f}$ noise generation processes. Therefore, I write the flickering source as the summation of $\mathrm{X}_{\mathrm{n}}(\mathrm{t})$.

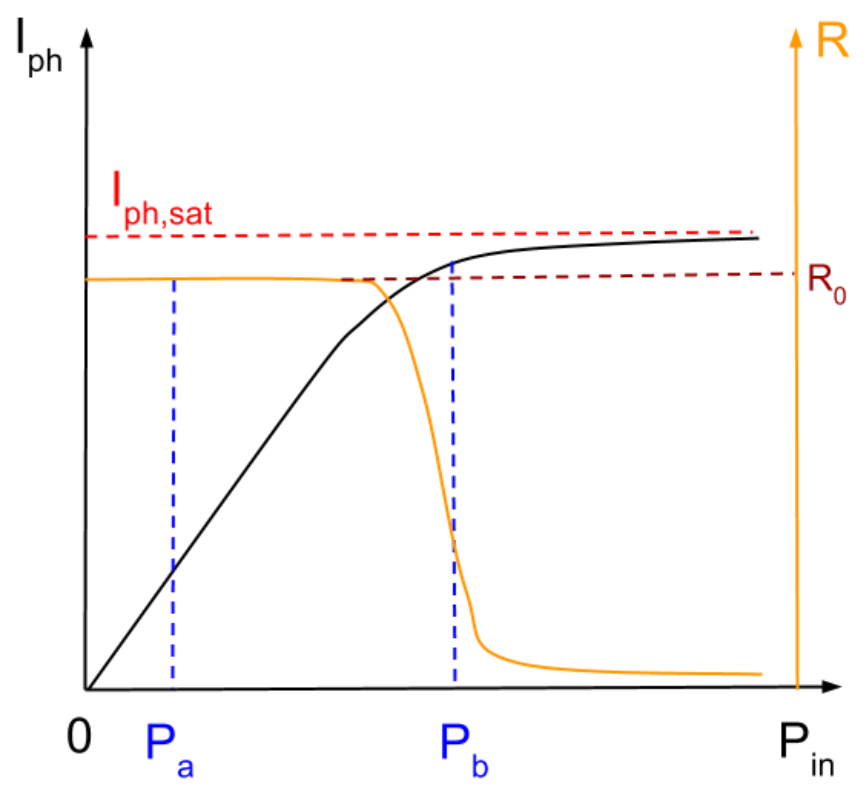

Figure 5.18: The photocurrent $I_{p h}$ (black solid line) and the mean of responsivity $R$ (orange solid line) as a function of input optical power $P_{\text {in }}$ in a typical photodiode. The $I_{p h \text { sat }}$ is the saturation photocurrent. $R_{0}$ is the small-signal responsivity. $P_{a}$ and $P_{b}$ are the operating points in the linear region and non-linear region.

To isolate the influence of the noise associated with the input (such as laser RIN, mechanical vibration of fibers, etc.), we ideally assume that there is no noise in the input optical signal and the measurement is done in a very stable environment. For an amplitude-modulated laser input modulated by a noiseless external modulator, the input optical RF power can be written as 


$$
P_{\text {in }}=P_{0}\left[1+m \cos \left(\omega_{0} t+\varphi_{0}\right)\right]
$$

where $\mathrm{P}_{0}$ is the average power, $\mathrm{m}$ is the modulation index, $\omega_{0}$ is the RF carrier frequency (not the optical carrier frequency), and $\varphi_{0}$ is the constant phase. The resulting photocurrent $\mathrm{I}_{\mathrm{ph}}$ is

$$
\begin{gathered}
I_{p h}=R P_{\text {in }} \\
=R_{0} P_{0}\left[1+m \cos \left(\omega_{0} t+\varphi_{0}\right)\right]\left[1+\sum_{n} X_{n}(t)\right] \\
=R_{0} P_{0}\left[1+m \cos \left(\omega_{0} t+\varphi_{0}\right)+\sum_{n} X_{n}(t)\right. \\
\left.+m \sum_{n} X_{n}(t) \cos \left(\omega_{0} t+\varphi_{0}\right)\right]
\end{gathered}
$$

Note that the first two terms in the square parentheses of Equation ( 5.14) are the deterministic response, the third term is the $1 / \mathrm{f}$ noise near $\mathrm{DC}$, and the fourth term is the up-converted $1 / \mathrm{f}$ noise. To make this more clear, the fourth term can be expanded with trigonometric identity and written as

$$
I_{p h, \text { noise }}=R_{0} P_{0} m \sum_{n} X_{n}(t) \cos \left(\omega_{0} t+\varphi_{0}\right)
$$

Therefore, the flicker noise near DC is converted to the amplitude noise near $\omega_{0}$.

We should also consider the large-signal condition when the photoresponse is no longer linear (e.g., operating point $\mathrm{P}_{\mathrm{b}}$ Figure 5.18). The difference is that the responsivity becomes timevarying as the input RF signal oscillates, which can be further expanded in Fourier series as

$$
R\left(P_{\text {in }}(t)\right)=\sum_{k=1}^{\infty}\left[U_{k} \cos \left(k \omega_{0} t\right)+V_{k} \sin \left(k \omega_{0} t\right)\right]\left[1+\sum_{n} X_{n}(t)\right]
$$


where

$$
U_{k}=\frac{2}{T} \int_{-T / 2}^{T / 2} R\left(P_{\text {in }}(t)\right) \cos \left(k \omega_{0} t\right) d t
$$

and

$$
V_{k}=\frac{2}{T} \int_{-T / 2}^{T / 2} R\left(P_{\text {in }}(t)\right) \sin \left(k \omega_{0} t\right) d t
$$

The resulting photocurrent becomes

$$
\begin{gathered}
I_{p h}=P_{0} \sum_{k=1}^{\infty}\left[U_{k} \cos \left(k \omega_{0} t\right)+V_{k} \sin \left(k \omega_{0} t\right)\right]\left[1+\sum_{n} X_{n}(t)\right][1 \\
\left.\quad+m \cos \left(\omega_{0} t+\varphi_{0}\right)\right] \\
=P_{0} \sum_{k=1}^{\infty}\left[U_{k} \cos \left(k \omega_{0} t\right)+V_{k} \sin \left(k \omega_{0} t\right)\right]\left[1+m \cos \left(\omega_{0} t+\varphi_{0}\right)\right. \\
\left.+\sum_{n} X_{n}(t)+m \sum_{n} X_{n}(t) \cos \left(\omega_{0} t+\varphi_{0}\right)\right] .
\end{gathered}
$$

The photonoise term in Equation ( 5.19) is

$$
\begin{aligned}
I_{\text {ph,noise }}=P_{0} \sum_{k=1}^{\infty} & {\left[U_{k} \cos \left(k \omega_{0} t\right)+V_{k} \sin \left(k \omega_{0} t\right)\right]\left[\sum_{n} X_{n}(t)\right.} \\
& \left.+m \sum_{n} X_{n}(t) \cos \left(\omega_{0} t+\varphi_{0}\right)\right]
\end{aligned}
$$

Although it is tedious to fully expand Equation ( 5.20), it is very clear that there are three sources that contribute to the fundamental frequency, $\omega_{0}$, in the output. The first one is the RF 
input modulated at $\omega_{0}$, the second is the $\omega_{0}$ Fourier component of the responsivity, and the third is the lower frequency component at $\omega_{0}$ after the mixing of the RF input and the $2 \omega_{0}$ Fourier component ( $2^{\text {nd }}$ harmonic) of the responsivity. The mathematical form of the noise near $\omega_{0}$ can be written as

$$
\begin{aligned}
I_{p h, \text { noise } \omega_{0}=} P_{0} & \sum_{n} X_{n}(t)\left[W_{1} \cos \left(\omega_{0} t+\theta_{1}\right)+m U_{0} \cos \left(\omega_{0} t+\varphi_{0}\right)\right. \\
& \left.+\frac{W_{2}}{2} \cos \left(\omega_{0} t+\theta_{2}-\varphi_{0}\right)\right]
\end{aligned}
$$

where

$$
\begin{gathered}
W_{k}=\sqrt{U_{k}^{2}+V_{k}^{2}} \\
\theta_{k}=-\arctan \left(\frac{V_{k}}{U_{k}}\right)
\end{gathered}
$$

Therefore, in the large-signal condition, the low-frequency noise near DC is also translated to the amplitude noise in higher frequency, but with more complicated weights than the small-signal condition.

However, more study is still needed to understand the details of such up-conversion. For example, for white noise such as thermal noise and shot noise, it is believed that half of the noise power is converted to amplitude noise and the other half goes to phase noise [155]. However, it is still not clear whether such an even split is justified for $1 / \mathrm{f}$ noise since $1 / \mathrm{f}$ noise is not white. Future study is still needed to have more thorough understanding on this subject.

\subsection{Summary}

In this chapter, I briefly review the noise in semiconductor devices and then focus on the lowfrequency noise in organic solar cells and high-power photodiodes. I demonstrate that low- 
frequency noise spectroscopy is a useful approach to understanding the underlying physics in organic solar cells and photodiodes.

I have studied the low-frequency noise characteristics of P3HT:PCBM bulk heterojunction organic solar cells in the dark condition. I found that the relationship between the current noise power spectral density and DC current level could be well explained by the competition between the recombination current noise and tunneling current noise. The interpretation of the noise characteristics is consistent with previous knowledge on the performance of the devices annealed at different temperatures.

I have also measured the LFN of commercial photodiodes and MUTC4 photodiodes with a balanced photodetection setup in dark and illuminated conditions. The measured photo-LFN from MUTC4 photodiodes under reverse bias decreases with increasing photogeneration, and we attribute such anomaly to the noise generation dominated by the trap-assisted tunneling.

In the end, I consider the up-conversion of the $1 / \mathrm{f}$ noise near DC to high frequency in a photodiode, and discussed the small-signal and large-signal scenarios. 


\section{Chapter 6 : Resonance-Coupled Photoconductivity Decay Measurement}

\subsection{Contactless Characterization of Semiconductor Materials}

The carrier lifetime is another fundamental physical parameter that is very crucial to device performance. Various methods, such as transient photoconductivity [28], transient photovoltage [78], and impedance spectroscopy [79], have been used to measure carrier recombination lifetimes. However, many of those methods require metal contacts that can be electrically probed in the experiments. Contactless characterization removes such limitation, thus it not only reduces the device fabrication cost, but also avoids the experimental artifacts related to metal contacts. Like many other semiconductor characterization techniques, there are DC and non-DC (i.e., AC and transient) contactless methods [156]. One of the most widely used DC contactless characterization methods is the Hall effect measurement, which can measure the carrier mobility and carrier density inside a semiconductor slab. Some DC contactless methods such as photomagnetoelectric sensing [157] can also measure carrier lifetimes based on indirect electromagnetic models.

Non-DC contactless methods have the advantage of directly measuring the carrier lifetimes, due to their capability to sense the carrier dynamics in time. There are several ways to extract the carrier recombination lifetimes with contactless methods, including but not limited to the following:

- Transient photoluminescence is a contactless method that has optical signals as both input and output. It is a very widely used method to measure the characteristic lifetime of materials including but not limited to semiconductors. Experimentally, it employs an optical input that is absorbable and stimulates the material system to an excited state, followed by a natural decay to the original state. During the decay process, some of the energy is lost in the form of radiative recombination, which leads to photoluminescence that can be detected by a photodetector. The lifetime associated with the optical decay is the recombination lifetime.

- Surface photovoltage measurement [158], [159]. This method senses the temporal surface 
voltage change of a semiconductor upon the excitation of a pulsed light. This technique has been commercialized in the semiconductor industry to characterize the minority carrier lifetime in electronic devices such as MOSFETs. However, extra care such as special surface cleaning before the measurement, the complexity of the vibrating surface voltage sensing probe (for the purpose of enhancing signal-to-noise ratio), and the ambiguity on distinguishing the surface recombination from bulk recombination, limit the wide application of this method.

- Time-resolved microwave conductivity (TRMC) measurement [160], [161]. In this method, a slab of semiconductor is placed inside a microwave waveguide and continuously excited by a background microwave signal. A power detector also constantly monitors the reflected microwave power from the semiconductor slab. This permits the reflected microwave power decay induced by the transient conductivity change in the semiconductor slab after being excited by a light pulse, and the recombination lifetime to be determined. Figure 6.1 shows a typical TRMC experimental setup.

- Resonance-coupled photoconductivity decay (RCPCD) measurement [162]. The idea of RCPCD is quite similar to RCPCD except that the background microwave signal is coupled to the semiconductor by an inductive coil. Details of this method will be discussed later in this chapter. 


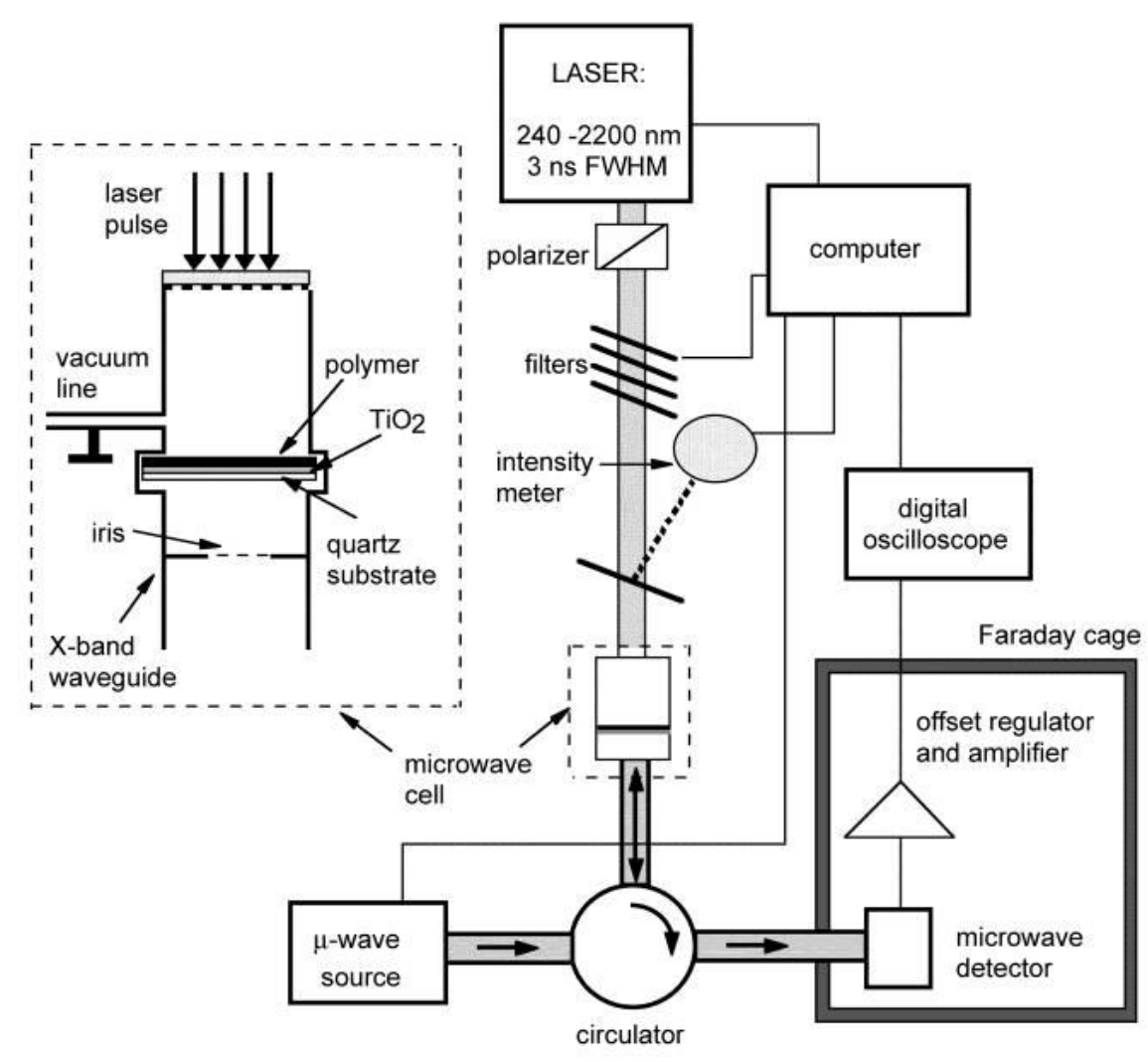

Figure 6.1: A typical experimental setup for time-resolved microwave conductivity measurement. The image is from Ref [161].

\subsection{Resonance-Coupled Photoconductivity Decay Measurement}

The work below resulted from the collaboration with Weikle's group here at UVA.

The resonance-coupled photoconductivity decay (RCPCD) measurement is a contactless method to characterize the carrier lifetime in wafers and thin films [162]. The fact that it is contactless is beneficial for lifetime measurements for some materials because contact interfaces have non-negligible influence on the thin active layer [163].

The RCPCD measurement is essentially homodyn RF detection. Figure 6.2 shows the circuit block diagram for this type of measurement, and Figure 6.3 is a picture of the RCPCD setup in the lab. The incident RF wave is split into two branches by a power splitter and amplified by two RF amplifiers (MiniCircuits ZHL-2010, 20dB gain). The RF wave in the upper branch in Figure 
6.2 is diverted by the $10 \mathrm{~dB}$ directional coupler (MiniCircuits ZEDC-10-2B, 30dB directivity) to excite the resonance circuit with the contactless load. The reflected wave is fed into the RF amplifier (MiniCircuits ZHL-6A, $25 \mathrm{~dB}$ gain) before the mixer (MiniCircuits ZAY-2, $23 \mathrm{dBm}$ input power limit). Two high-Q variable piston capacitors (Johanson Technology), $\mathrm{C}_{1}$ and $\mathrm{C}_{2}$, are used to tune the circuit to the resonance point and $\mathrm{L}_{1}$ is a solenoid that provides a physical interface with the contactless load. When a light pulse excites the organic film, the conductivity change will induce a change in the reflected power from the resonance circuit until the film returns to the steady state. The transient change is then demodulated by the mixer and measured by the oscilloscope. A transient signal related to the conductivity decay will be detected, and the recombination lifetime can be extracted. Compared with other contactless methods such as the time-resolved microwave conductivity measurement [160], RCPCD measurement features a more compact setup and a more flexible tuning mechanism due to the absence of metal waveguides.

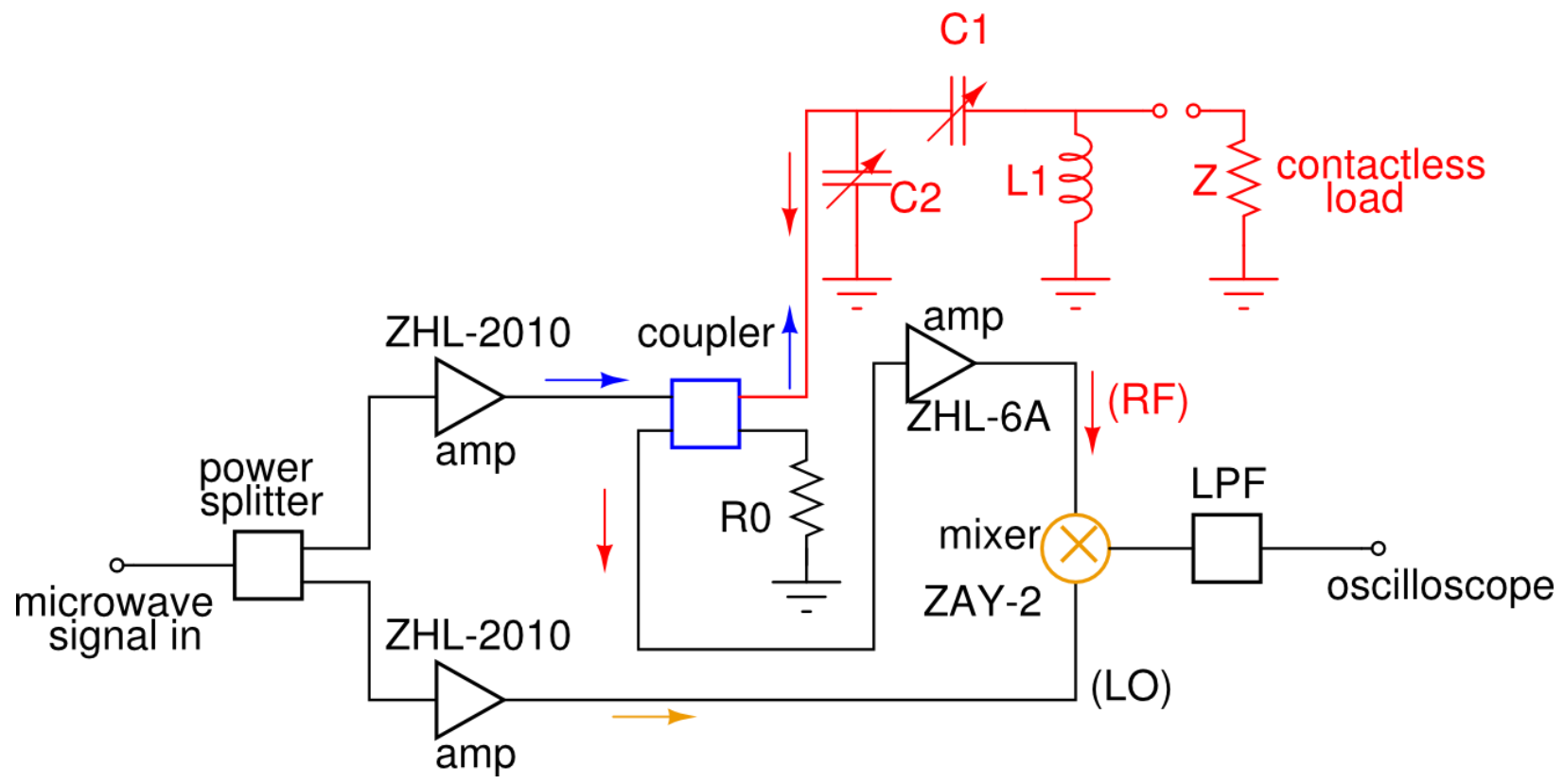

Figure 6.2: The circuit block diagram of RCPCD measurement. The impedance of the contactless load is subject to change upon the photoexcitation. 


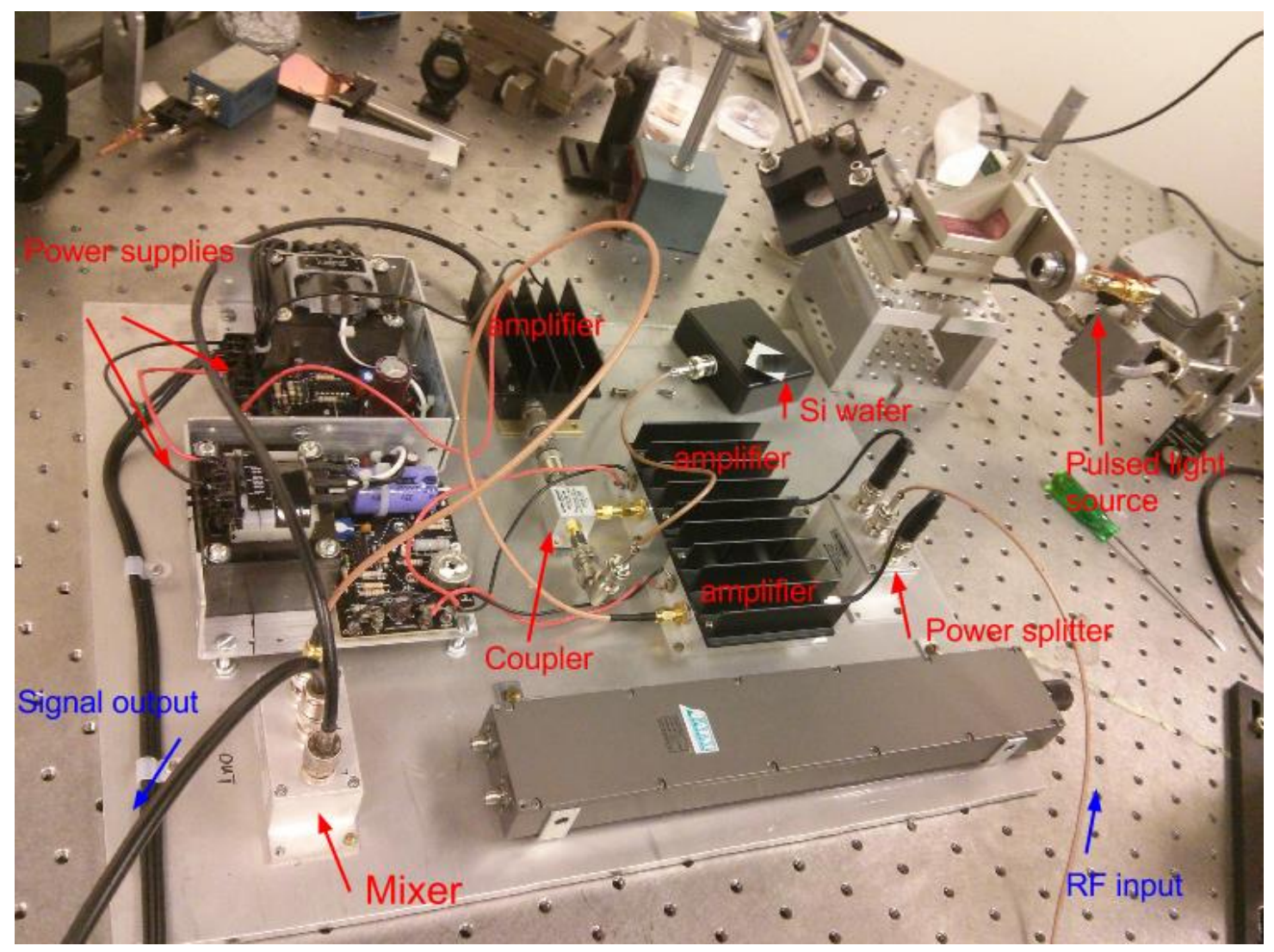

Figure 6.3: The RCPCD setup with a single mixer.

The most critical part of the whole setup is the resonance circuit, which is hidden in the black box under the silicon wafer in Figure 6.3. An illustration of the resonance circuit without the tuning capacitors is shown in Figure 6.4. This configuration is inspired by the principles of helical antennas [164]. When the circuit is excited by an AC voltage source, there is a timevarying magnetic field in the solenoid, which further induces an $\mathrm{AC}$ electrical field around the solenoid. Part of the electrical field is coupled to the nearby dielectric slab, and interacts with the mobile charge carriers in the slab. Therefore, the change in the number of the mobile carriers caused by the photogeneration in the slab will influence the magnetic field in the solenoid. This, in turn, influences the resonance circuit. Since carriers cannot escape through sweep-out in the dielectric slab, the excess photogenerated carriers can only relax by recombination, resulting in an exponential decay of the conductivity: 


$$
\sigma(t)=\sigma_{\infty}+\left(\sigma_{0}-\sigma_{\infty}\right) \exp \left(-\frac{t}{\tau}\right)
$$

where $\sigma_{0}$ is the conductivity at $\mathrm{t}=0, \sigma_{\infty}$ is the conductivity in steady state, and $\tau$ is the recombination lifetime.

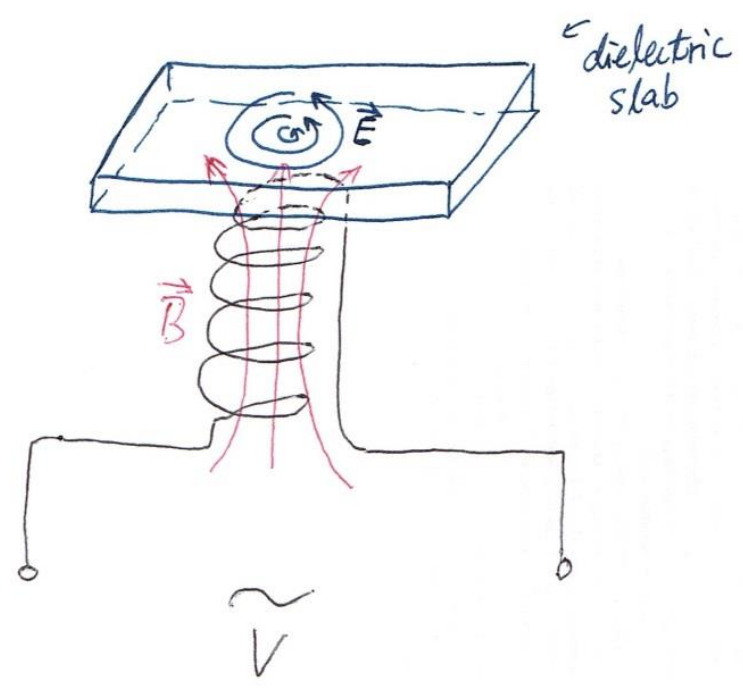

Figure 6.4: An illustration of the solenoid with a dielectric slab (e.g. silicon wafer) on the top in the $R C P C D$ resonance circuit.

The mathematical description of the frequency mixing in Figure 6.2 can be understood as follows. In the steady state, the local oscillator (LO) signal after the ZHL-2010 amplifier and the RF signal after the ZHL-6A amplifier can be written as Equations ( 6.2) and ( 6.3), respectively:

$$
\begin{aligned}
& V_{L O, S S}(t)=A_{L O} \cos \left(\omega_{0} t+\theta_{L O}\right) \\
& V_{R F, S S}(t)=A_{R F} \cos \left(\omega_{0} t+\theta_{R F}\right)
\end{aligned}
$$

where $\left.A_{\{L O}, R F\right\}$ and $\theta_{\{L O, R F\}}$ are the steady-state voltage amplitude and phase of $L O$ and $R F$ signals. The IF output from the mixer is 


$$
\begin{aligned}
V_{I F, S S}(t)=C V_{R F, S S}(t) V_{L O, S S}(t) \\
\quad=\frac{C A_{R F} A_{L O}}{2}\left[\cos \left(\theta_{R F}-\theta_{L O}\right)+\cos \left(2 \omega_{0} t+\theta_{L O}+\theta_{L O}\right)\right] .
\end{aligned}
$$

The constant, C, in Equation ( 6.4) stands for the mixer conversion loss. The low-pass filter after the mixer removes the high-frequency component, leaving the detected signal to be

$$
V_{\text {out }, s S}(t)=\frac{C A_{R F} A_{L O}}{2} \cos \left(\theta_{R F}-\theta_{L O}\right) .
$$

Therefore, one should see a DC voltage offset on the oscilloscope in the steady state, unless the phase difference between the RF and $\mathrm{LO}$ is $90^{\circ}$, or the reflected RF signal is close to zero when the LC circuit is well matched.

When the silicon wafer is excited by a pulsed light source, the RF signal into the mixer undergoes a change described by

$$
V_{R F}(t)=\left[A_{R F}+a(t)\right] \cos \left[\omega_{0} t+\theta_{R F}+\phi(t)\right]
$$

where a(t) and $\varphi(t)$ are the transient response of voltage amplitude and phase. Therefore, the corresponding output after the low pass filter can be written as

$$
V_{\text {out }}(t)=\frac{C\left[A_{R F}+a(t)\right] A_{L O}}{2} \cos \left[\theta_{R F}-\theta_{L O}+\phi(t)\right] .
$$

Equation ( 6.7) gives the mathematical behavior of the signal measured by the oscilloscope.

To carry out the transient measurement, a pulsed light source was constructed with an Opnex red laser diode (HL6362MG, 640nm, $45 \mathrm{~mW}$ ), and a 2N3904 NPN bipolar junction transistor (BJT) as shown in Figure 6.5. The base of the BJT was driven by an Agilent 81110A pulse generator which outputted voltage pulses that switched between $2.4 \mathrm{~V}$ and $0 \mathrm{~V}$, with pulse width 
of $20 \mu \mathrm{s}$, repetition rate of $100 \mathrm{~Hz}$ (duty cycle of $0.2 \%$ ), and lead time of $2 \mathrm{~ns}$. Since the BJT only had a finite gain-bandwidth product, the real speed of the optical output from the laser diode needed to be characterized. A Hamamatsu silicon photodiode with bandwidth of $2 \mathrm{GHz}$ was used to measure the optical pulses from the laser diode, and the photocurrent was amplified by a commercial high-speed transimpedance amplifier (Femto DHPCA-100, 200MHz bandwidth, 1.8 ns rise/fall time, $1000 \mathrm{~V} / \mathrm{A}$ sensitivity). According to Figure 6.5(b) and Figure 6.5(c), the fall time was measured to be near $35 \mathrm{~ns}$ while the rise time is about $250 \mathrm{~ns}$. Both measured rise time and fall time are at least one order of magnitude larger than the rise/fall time of the amplifier, which indicates that the measurement was not limited by the bandwidth of the amplifier.

A piece of $500 \mu \mathrm{m}$-thick high-resistivity silicon wafer was used to test the functionality of this system. The silicon wafer was loaded onto the inductive coil and the whole system was tuned to resonate at $327 \mathrm{MHz}$ by monitoring the $\mathrm{S}_{11}$ parameter in a network analyzer: the $\mathrm{S}_{11}$ exhibits a minimum when the system resonates. Also, the larger the $\mathrm{Q}$ factor of the resonance, the higher the sensitivity of the system. After setting the input microwave frequency to the resonance point, the pulsed light source was turned on to excite the silicon wafer. The IF output from the mixer was first low-pass filtered to remove the residual RF and LO signals, and measured by an Agilent Infiniium DSO81004B high-speed oscilloscope with input impedance of 50 Ohm. 


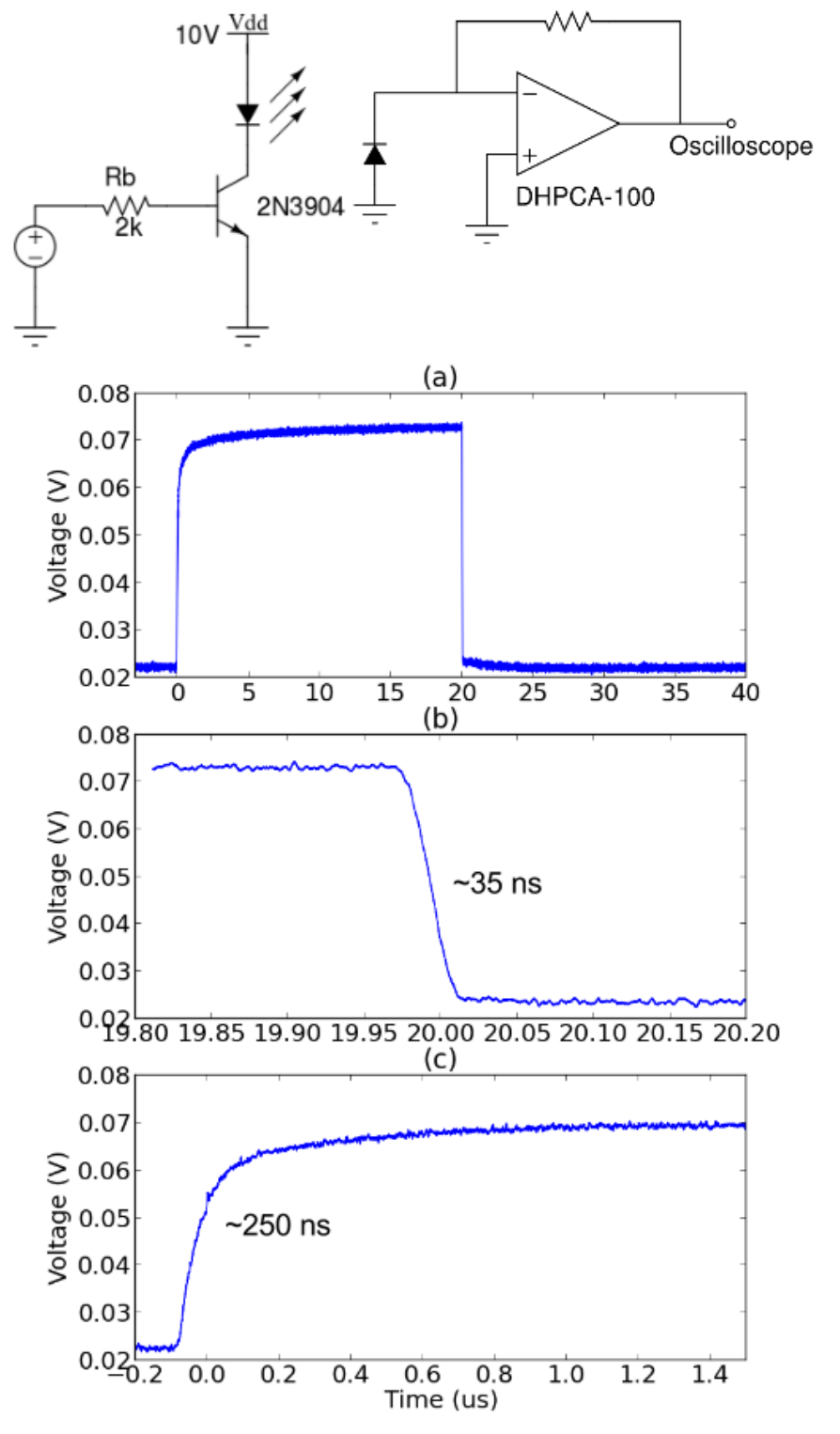

Figure 6.5: The driving and measurement circuits of the pulsed light source, and the photocurrent response of the (a) complete pulse, (b) the falling edge of the pulse and (c) the rising edge of the pulse. 
(a)

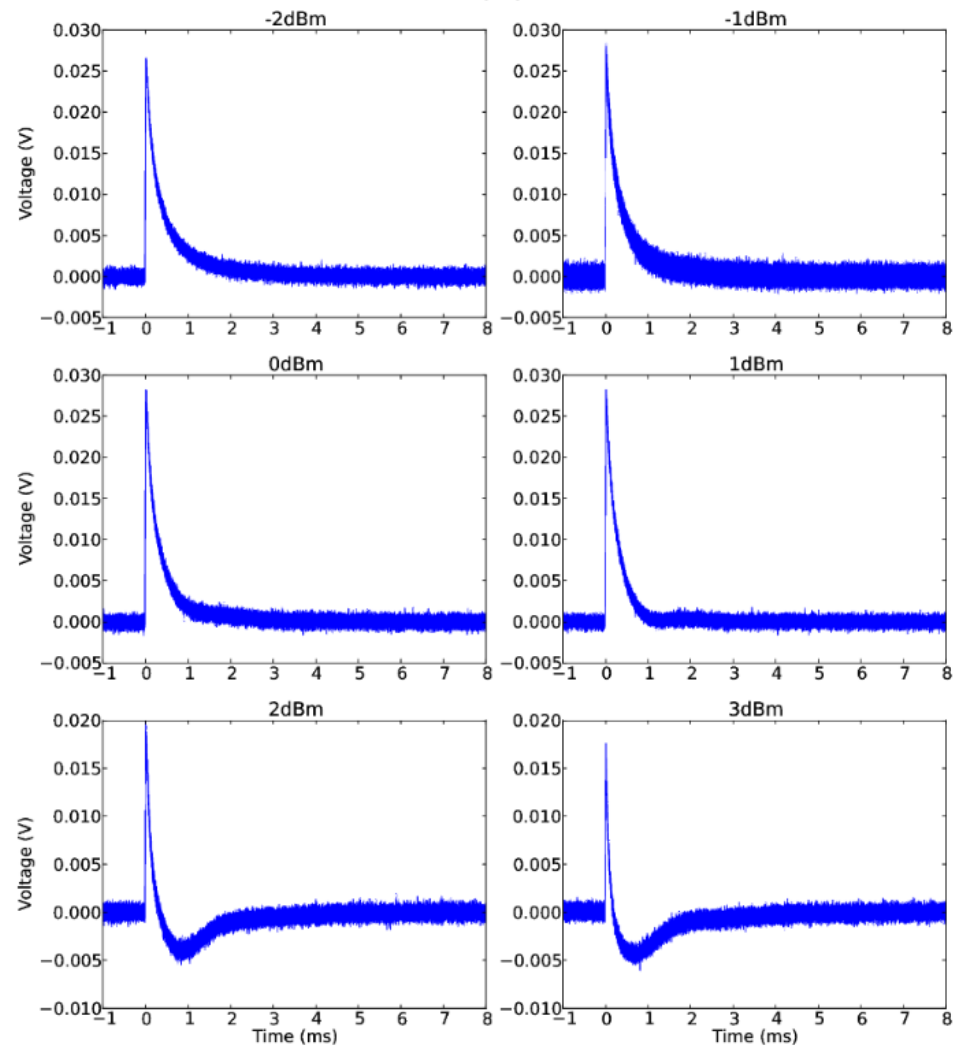

(b)

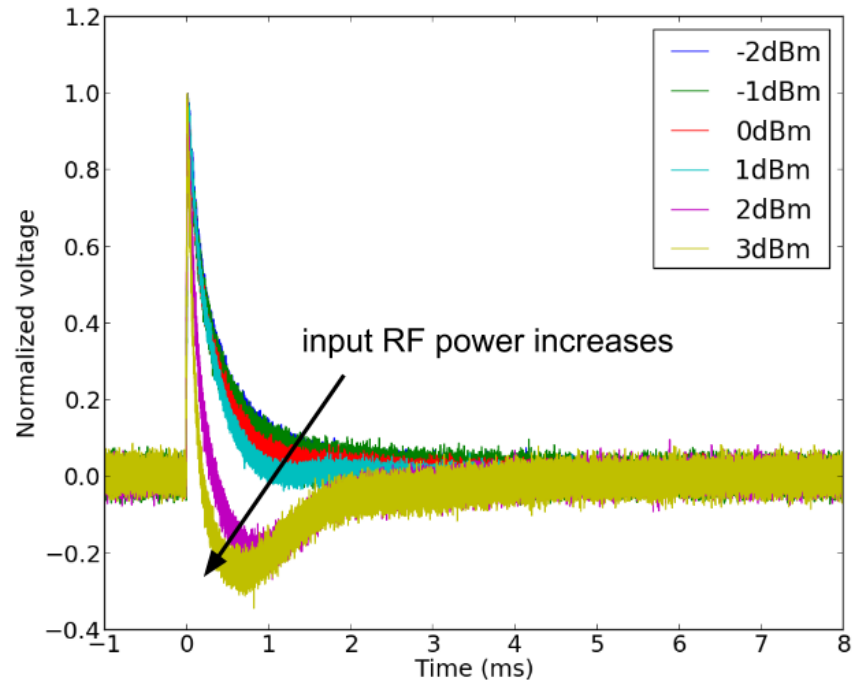

Figure 6.6: (a) The absolute and (b) normalized transient $R F$ response induced by the conductivity of the high-resistivity silicon wafer at different input $R F$ power levels. The DC offsets are all subtracted already.

Figure 6.6 shows the RCPCD response of the high-resistivity silicon wafer as a function of the input RF power. It is clear that the decay behavior changes once the input RF power exceeds - 
$1 \mathrm{dBm}$. As the RF power further increases above $1 \mathrm{dBm}$, undershoot behavior appears in the response that was not seen at lower RF power levels. This is very likely an artifact related to the linearity of the mixer. At the high power level, the higher-order harmonics of the RF and LO channels become non-negligible. Those harmonics not only distort the signal on the fundamental frequency, but also mix with each other, and the down-converted signal can interfere with the baseband signal from the fundamental frequency. The exact details in this process are complex. But as a rule of thumb, this makes the input power levels of the LO and the RF to the mixer high enough so that the transient response can be detected, but also low enough to avoid the distortion from higher-order harmonics. From Figure 6.6, we estimate the recombination lifetime of the silicon wafer under test to be approximately $0.3 \mathrm{~ms}$.

\subsection{Improved RCPCD Measurement with IQ Mixing}

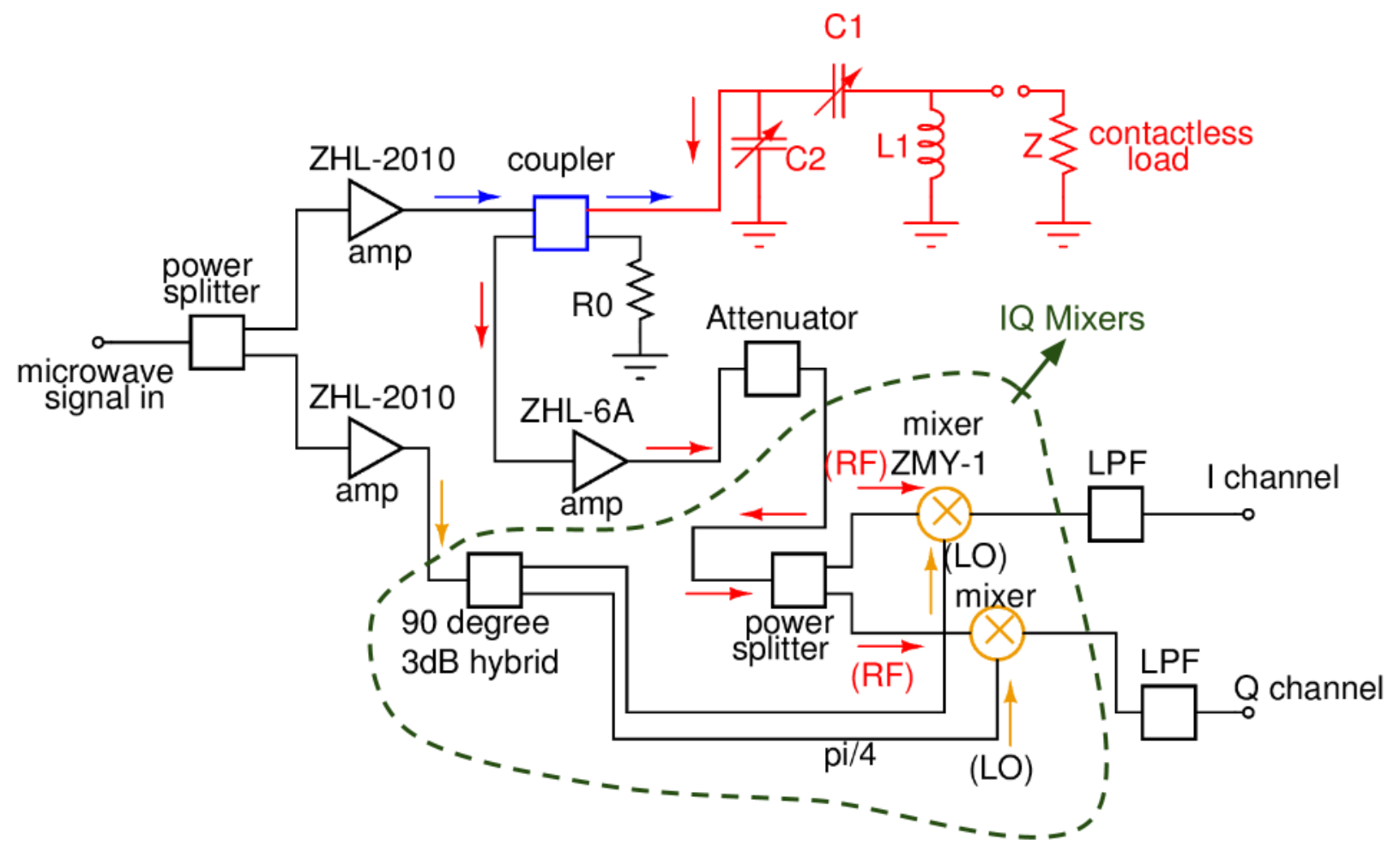

Figure 6.7: The circuit block diagram of improved RCPCD measurement with IQ mixers. The part inside the dashed circle lines is the implementation of IQ mixers using discrete components. 
From Equation ( 6.7), it is obvious that the transient responses of both amplitude and phase influence the decay shape on the oscilloscope. The problem with the RCPCD implementation described in the last section is that it cannot separate the amplitude information a(t) from the phase $\varphi(t)$ in Equation ( 6.7). Also, $V_{\text {out }}(t)$ is not linearly dependent on $\varphi(t)$, which raises questions about the accuracy of the extracted carrier recombination lifetime solely based on Equation ( 6.7).

This limitation can be resolved by introducing IQ mixing to the RCPCD measurement. IQ mixers are made up of two identical mixers with $90^{\circ}$ phase difference in either RF or LO input. Figure 6.7 shows the improved RCPCD setup with IQ mixing. The RF signal after the RF amplifier ZHL-6A and attenuator is split into two identical branches, which are fed into two mixers, respectively. On the other hand, the LO signal is split into two branches with $90^{\circ}$ phase difference after passing through a $90^{\circ} 3 \mathrm{~dB}$ coupler, and then into two mixers. The branch with no phase shift is called the "I" (in-phase) channel, while the branch with $90^{\circ}$ phase shift is the "Q" (quadrature-phase) channel. The RF signals into both the I and Q mixers are identical, and can be written as

$$
V_{R F, I}(t)=V_{R F, Q}(t)=\left[A_{R F}+a(t)\right] \cos \left[\omega_{0} t+\theta_{R F}+\phi(t)\right]
$$

However, the LO signals are different. They are

$$
V_{L O, I}(t)=A_{L O} \cos \left(\omega_{0} t+\theta_{L O}\right)
$$

and

$$
V_{L O, Q}(t)=A_{L O} \cos \left(\omega_{0} t+\theta_{L O}+\frac{\pi}{4}\right)=A_{L O} \sin \left(\omega_{0} t+\theta_{L O}\right)
$$


The IF output from the I mixer is

$$
\begin{gathered}
V_{I F, I}(t)=C V_{R F, I}(t) V_{L O, I}(t) \\
=\left[A_{R F}+a(t)\right] A_{L O} \cos \left[\omega_{0} t+\theta_{R F}+\phi(t)\right] \cos \left(\omega_{0} t+\theta_{L O}\right) \\
=\frac{C\left[A_{R F}+a(t)\right] A_{L O}}{2}\left[\cos \left(\theta_{R F}-\theta_{L O}+\phi(t)\right)+\cos \left(2 \omega_{0} t+\theta_{L O}+\theta_{L O}+\phi(t)\right)\right]
\end{gathered}
$$

where $\mathrm{C}$ is the mixer conversion loss. And the IF output from the Q mixer is

$$
\begin{gathered}
V_{I F, Q}(t)=C V_{R F, Q}(t) V_{L O, Q}(t) \\
=\left[A_{R F}+a(t)\right] A_{L O} \cos \left[\omega_{0} t+\theta_{R F}+\phi(t)\right] \sin \left(\omega_{0} t+\theta_{L O}\right) \\
=\frac{C\left[A_{R F}+a(t)\right] A_{L O}}{2}\left[\sin \left(-\theta_{R F}+\theta_{L O}-\phi(t)\right)+\sin \left(2 \omega_{0} t+\theta_{L O}+\theta_{L O}+\phi(t)\right)\right] .
\end{gathered}
$$

After the low-pass filters, the baseband signals in the I and Q channels are

$$
V_{\text {out }, I}(t)=\frac{C\left[A_{R F}+a(t)\right] A_{L O}}{2} \cos \left[\theta_{R F}-\theta_{L O}+\phi(t)\right]
$$

and

$$
V_{\text {out }, Q}(t)=-\frac{C\left[A_{R F}+a(t)\right] A_{L O}}{2} \sin \left[\theta_{R F}-\theta_{L O}+\phi(t)\right]
$$

Equations ( 6.13) and ( 6.14) fully describe the RCPCD decay behavior to be measured by the oscilloscope, from which we can solve for the transient amplitude and phase using the following equations

$$
A(t)=\frac{C\left[A_{R F}+a(t)\right] A_{L O}}{2}=\sqrt{V_{\text {out }, I}^{2}(t)+V_{\text {out }, Q}^{2}(t)}
$$

and 


$$
\phi(t)+\theta_{R F}-\theta_{L O}=\arccos \left(\frac{V_{\text {out }, I}(t)}{\sqrt{V_{\text {out }, I}^{2}(t)+V_{\text {out }, Q}^{2}(t)}}\right) .
$$

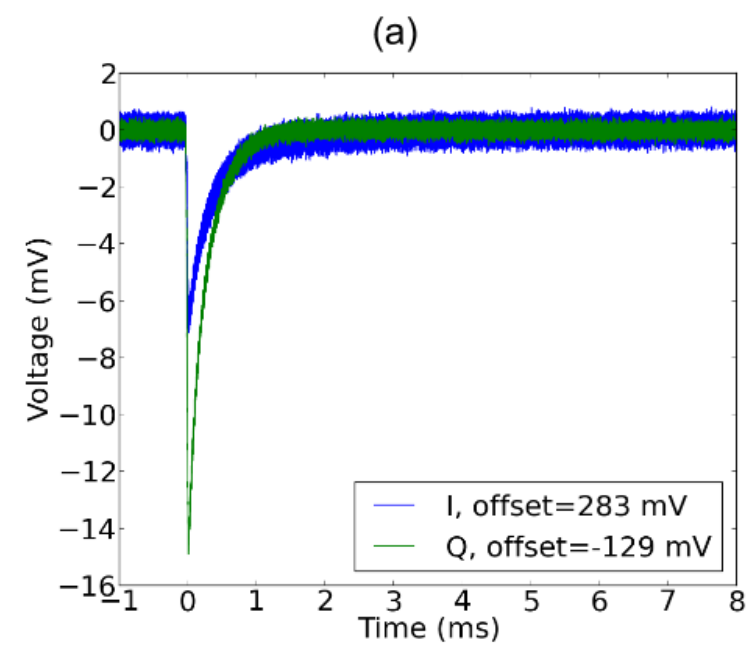

(b)
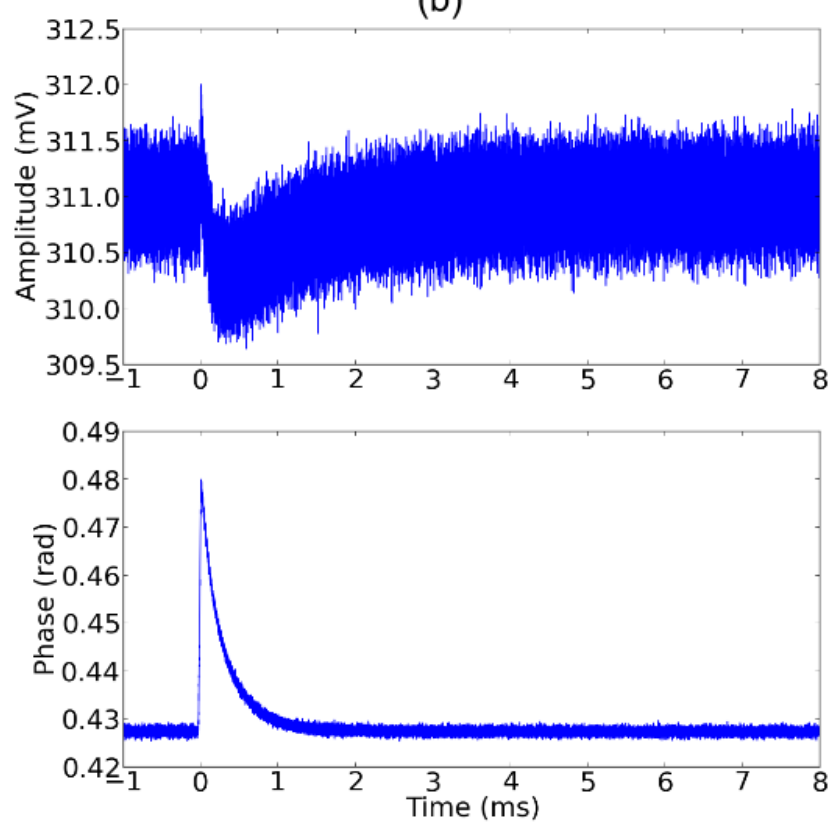

Figure 6.8: The transient response of (a) I and $Q$ channels and (b) the extracted amplitude and phase measured by the improved RCPCD method with IQ mixing.

Figure 6.8 shows the transient response of the same high-resistivity silicon wafer measured by the improved RCPCD with IQ mixing and with the same pulsed light source as the last section. 
In Figure 6.8(a) the DC offsets have been removed to highlight the delicate decay features since the I and Q channels have very different DC offsets. The extracted amplitude and phase transient responses are plotted in Figure 6.8(b). We can clearly see that the change in phase is much more significant than the amplitude, and the time constant extracted from the lower plot in Figure 6.8(b) is approximately $\sim 0.25 \mathrm{~ms}$, which is slightly smaller than the value of $0.3 \mathrm{~ms}$ measured by the single-mixer RCPCD method.

\subsection{Summary}

In conclusion, I have built an RF contactless characterization system for semiconductor wafers. I first adapted a single-mixer resonance-coupled photoconductivity decay technique, and further improved the accuracy of the results by replacing the single mixer with IQ mixers. This method can be a potentially useful real-time wafer characterization technique in semiconductor manufacturing facilities. 


\section{Chapter 7 : Future work}

\subsection{Phase Noise Measurement of High-Power Photodiodes with Phase- Sensitive Detection}

As shown in Chapter 5, the low-frequency noise near DC is a good indicator of the physical properties of charge carrier transport in MUTC4 photodiodes. However, most applications that utilize MUTC photodiodes do not operate near DC: usually they operate at frequencies above GHz. When using photodiodes to generate microwave oscillation, the phase noise is a very important metric to evaluate the overall performance. Similar to the noise spectra measured in Chapter 5, the phase noise spectrum consists of the white noise (usually dominated by the shot noise) and the $1 / \mathrm{f}$ noise. The $1 / \mathrm{f}$ noise can be especially detrimental to the oscillator owing to its proximity to the RF carrier frequency. One of the meaningful extensions of the work in Chapter 5 is measuring and understanding the $1 / \mathrm{f}$ phase noise of high-power photodiodes at high frequency.

Dr. Scott Diddams' group, our collaborators in NIST, has developed a sophisticated optoelectronic system to generate an ultrastable $10 \mathrm{GHz}$ microwave oscillation using photodiodes. An essential focus of that program is to characterize the photodiode phase noise and the AM-PM conversion factor [33], a metric that characterizes the coupling between the phase of the photocurrent and the amplitude of the optical input [7], [149]. Measuring AM-PM conversion is very important because the laser always has amplitude noise, which may have a $1 / \mathrm{f}$ component. Previously, the phase noise and AM-PM conversion factor of MUTC4 high-power photodiodes that were designed and fabricated by our group have been measured as part of the collaboration [33]. It was observed that the phase noise floor was $10 \mathrm{~dB}$ lower and the AM-PM conversion factor was smaller than commercial photodiodes at $10 \mathrm{GHz}$. The MUTC4 photodiodes were also able to handle larger photocurrents.

One project for further collaboration with NIST would be to extend the measurement of phase noise (especially 1/f noise) and AM-PM conversion to other MUTC photodiodes that were designed with different performance goals. For example, our group has designed and fabricated MUTC5 photodiodes for the operation above $50 \mathrm{GHz}$, by reducing the thickness of the active 
layers compared with the MUTC4 structure [165]. MUTC10 photodiodes with even thinner layers than MUTC5 photodiodes have been designed for the operation above $70 \mathrm{GHz}$. Two separate experiments are needed to measure the phase noise and the AM-PM conversion factor. Figure 7.1 (a) illustrates the NIST apparatus for low phase noise $10 \mathrm{GHz}$ microwave generation using photodiodes, and Figure 7.1 (b) shows the simplified experimental block diagram. A stable mode-locked laser with pulse width less than $1 \mathrm{ps}$ and repetition rate of $1 \mathrm{GHz}$ is used to excite photocurrent pulses in two photodiodes. The $10 \mathrm{GHz}$ electrical oscillation can be extracted by band-pass filtering the $10^{\text {th }}$ harmonic in the photocurrent. The random difference of the phase noise in two photodiodes is detected by homodyne mixing. Figure 7.2 shows a block diagram of the AM-PM conversion factor measurement [166]. An amplitude modulation is intentionally applied to the laser pulse train, and the corresponding phase change is detected. The AM-PM conversion factor is

$$
\alpha_{A M-P M}=\frac{\Delta \varphi}{\Delta P / P}
$$

where $\Delta \varphi$ is the phase change and $\Delta \mathrm{P} / \mathrm{P}$ is the normalized amplitude change. 
(a)

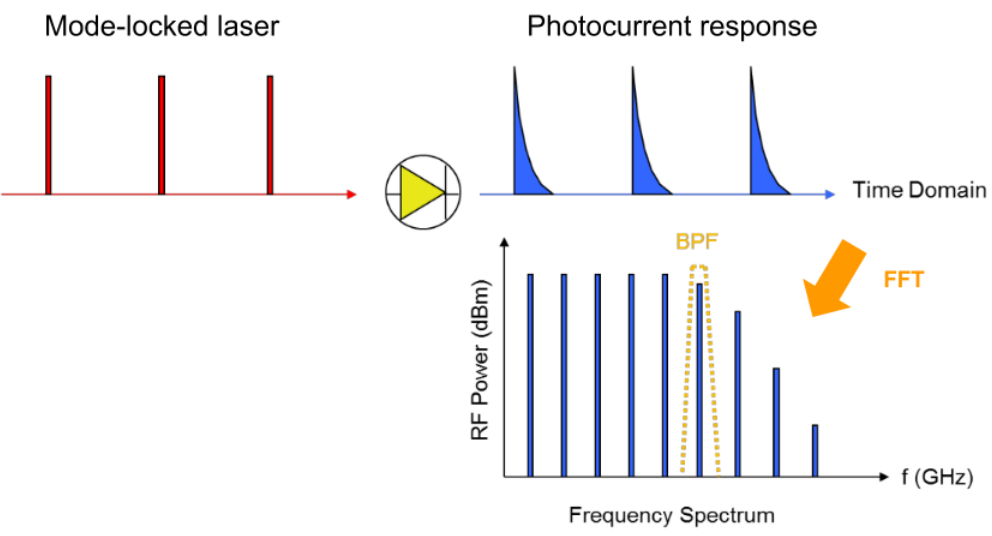

(b)

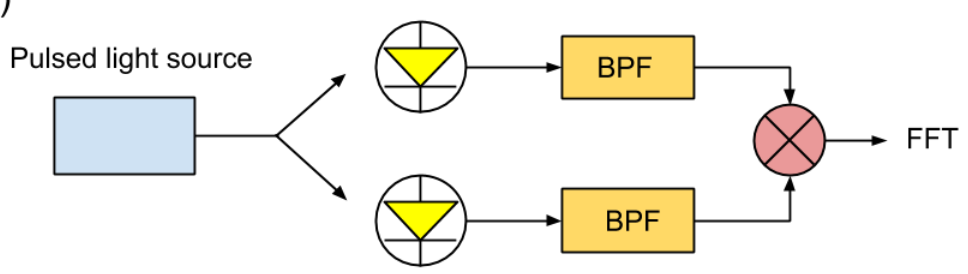

Figure 7.1: (a) Principle of optics-based microwave signal generation. Part of the figure is from Ref[33]. (b) A simplified block diagram of photodiode phase noise measurement.

A continuation of this work would be to build the in-house experiment apparatus described in Figure 7.1 and Figure 7.2 to measure the phase noise and AM-PM conversion factor of MUTC5 and MUTC10 photodiodes with help from NIST. This would enable further study the physical processes that contribute to the $1 /$ f noise near the RF carrier frequency, and device optimization in terms of better phase noise performance.

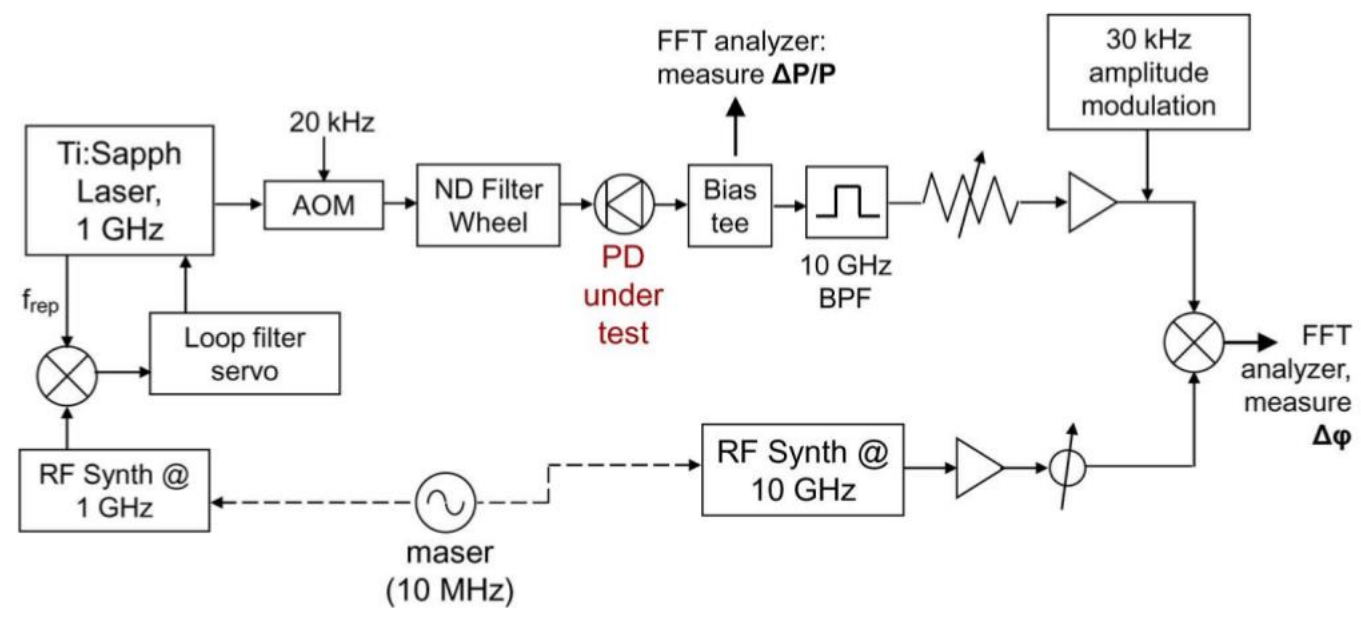

Figure 7.2: AM-PM conversion factor measurement. This figure is from Ref[166]. 


\subsection{Contactless Characterization of Organic Thin Films}

Contactless characterization is especially useful for studying BHJ solar cells because it provides a direct physical link to the exciton dynamics that is not easily accessible by other noncontactless methods. As described in Chapter 2, the mobile charge (free polaron) generation in BHJ solar cells is not only dependent on the exciton dissociation, but also related to the exciton generation and recombination. The quantum yield of the mobile charge generation, defined as the number ratio of mobile charges to incident photons, is found to be a sub-linear function of photoexcitation intensity in a plain P3HT film [167]. The exciton annihilation or exciton diffusion is accounted for the sub-linear dependence in the high photoexcitation intensity range. Moreover, the exciton diffusion length can be extracted if the sample is prepared with a bi-layer structure [168]. The impact of excess energy during the exciton generation on the mobile carrier generation can also be revealed by a wavelength-dependent photoexcitation study [169].

The RCPCD measurement described in Chapter 6 may also be applied to organic films. In practice, however, an organic film used in most organic electronics is too thin to provide enough absolute change in conductivity for RCPCD measurement. The film thickness is only tens to hundreds of $\mathrm{nm}$, more than three orders of magnitude thinner than the silicon wafer $(500 \mu \mathrm{m})$ tested in Chapter 6. One limitation of the resonance circuit (Figure 6.4) in Chapter 6 is that the field profile diverges very quickly near the end of the solenoid, and the profile cannot be easily controlled by the tuning circuit. The induced electric field near the end is much smaller than that in the middle of the solenoid. Moreover, the direction of the electric field near the end is not always in the plane of the semiconductor slab, the direction in which the mobile charges are driven by the electric field most easily. Therefore, the limited coupling between the contactless load and the RCPCD sensing circuit in Figure 6.4 may lead to unsatisfied results when the RCPCD method is applied to thin-film systems.

A system with enhanced tunability and load coupling is needed. Another system, inspired by the existing RCPCD setup and time-resolved microwave conductivity (TRMC) method [160], [161], [167] could be employed. This setup would only replace the resonance circuit in the 
improved RCPCD setup (red-colored part in Figure 6.7). The rest of the apparatus would be unchanged. The new resonance circuit is shown in Figure 7.3. A section of microwave waveguide would be used to form a resonance cavity together with the optically transparent RF reflector on the right, and the thin film sample would be mounted on the waveguide flange. The background microwave excitation will be coupled to the cavity from the left. By adjusting the wavelength of the microwave and the spacing between the right-end flange and the RF reflector, the system can be tuned to resonance, and have the electric field maximum on the thin film at the same time. After that, laser pulses from the right can excite the thin film through the optically transparent RF reflector, which can be a meshed metal grid or ITO sheet, inducing a transient change in the conductivity of the thin film. This will induce a transient change in the reflected microwave power, which can be detected by an external circuit.

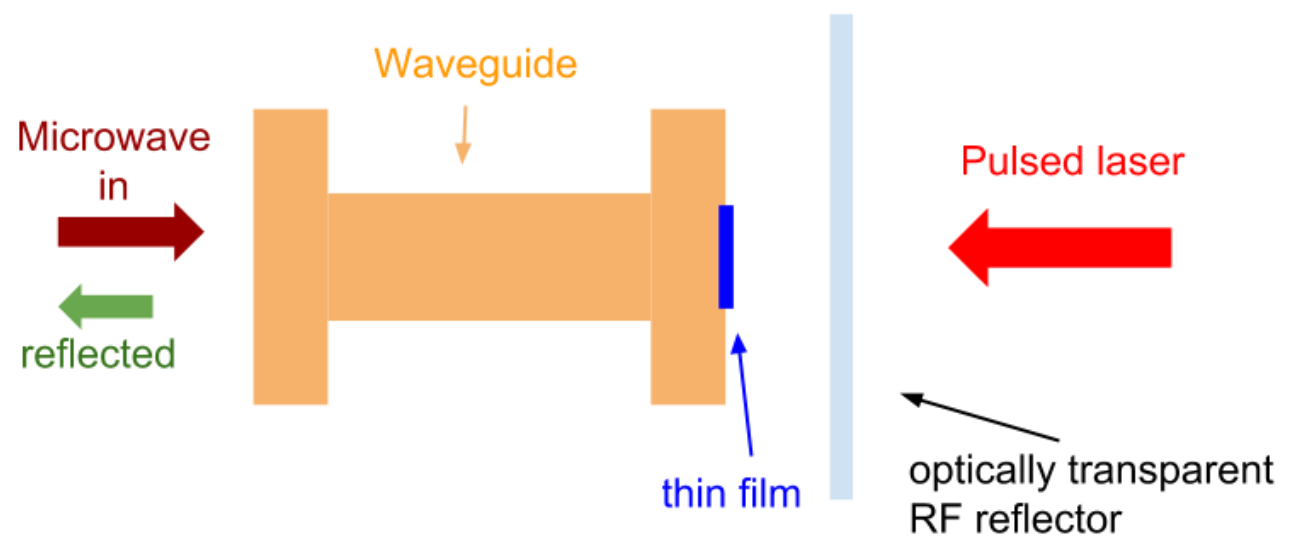

Figure 7.3: The improved resonance circuit.

Compared with the RCPCD resonance circuit in Chapter 6, the new circuit has finer control of the electric field and potentially higher Q factor (meaning greater sensitivity) because of the waveguide. Compared with the traditional TRMC setup described in Figure 6.1, the new setup is projected to have better tunability, and can also utilize the IQ mixing from the improved RCPCD setup for better result accuracy. Once the system is functional, it can be used to investigate the excitonic processes (e.g., exciton diffusion and recombination), which is beyond the capability of other non-contactless methods such as transient photoconductivity. 


\section{Appendix: Source Code for PICTS Data Processing}

Below is my MATLAB code for Tikhonov regularization with non-negativity constraint. The relationship between $I(t)$ and $N_{T}(f)$ is

$$
I(t)=C \int_{0}^{\infty} N_{T}(f) f \exp (-f t) d f
$$

where $\mathrm{I}(\mathrm{t})$ is the input data (e.g., experimental data), and $\mathrm{N}_{\mathrm{T}}(\mathrm{f})$ is the spectrum to be calculated in f-domain.

The regularization parameter can be either provided externally, or determined heuristically. Three different discretization schemes, i.e., linear, log, and Gauss-Laguerre, are available.

File list:

- test_tiknc.m: the test script for tikregnc.m

- tikregnc.m: function that solves for solutions to Tikhonov regularization with nonnegativity constraint

- ncsolve.m: linear non-negativty constrained problem solver; a linear programming solver is used

- get_A: get discretized matrix A; linear, log, Gaussian-Laguerre quadrature discretization are available

- $\quad$ _func: generates time-domain test data from a Gaussian spectrum in f-space

- gen_laguerre_rule2.m: modified Gauss-Laguerre quadrature discretizer based on the code at http://people.sc.fsu.edu/ jburkardt/m_src/gen_laguerre_rule/gen_laguerre_rule.html 
Other dependencies:

- regutools: a copy of P.C. Hansen's Regularization toolbox from

http://www2.imm.dtu.dk/ pcha/Regutools/

- MATLAB Optimization Toolbox http://www.mathworks.com/products/optimization/

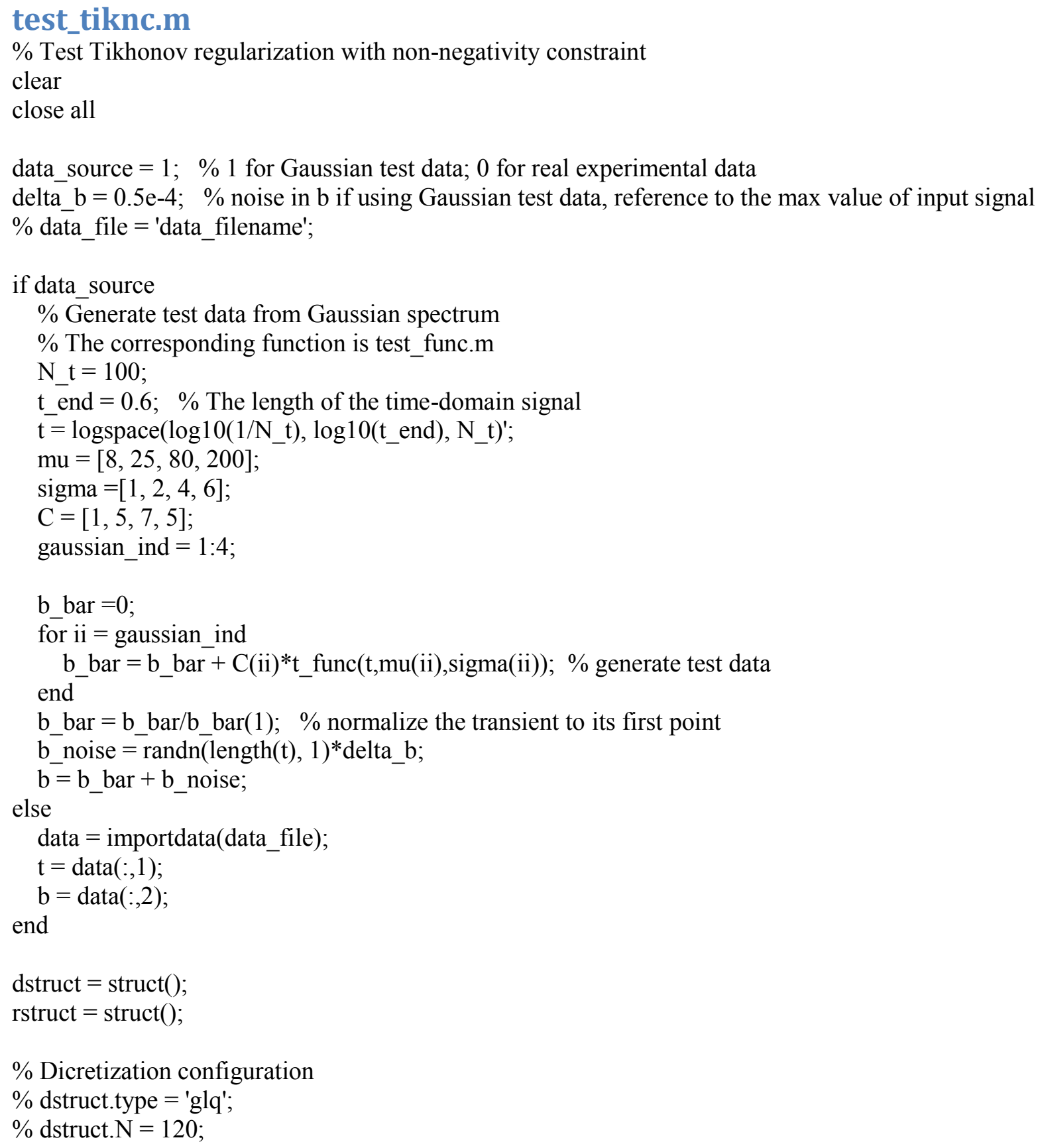




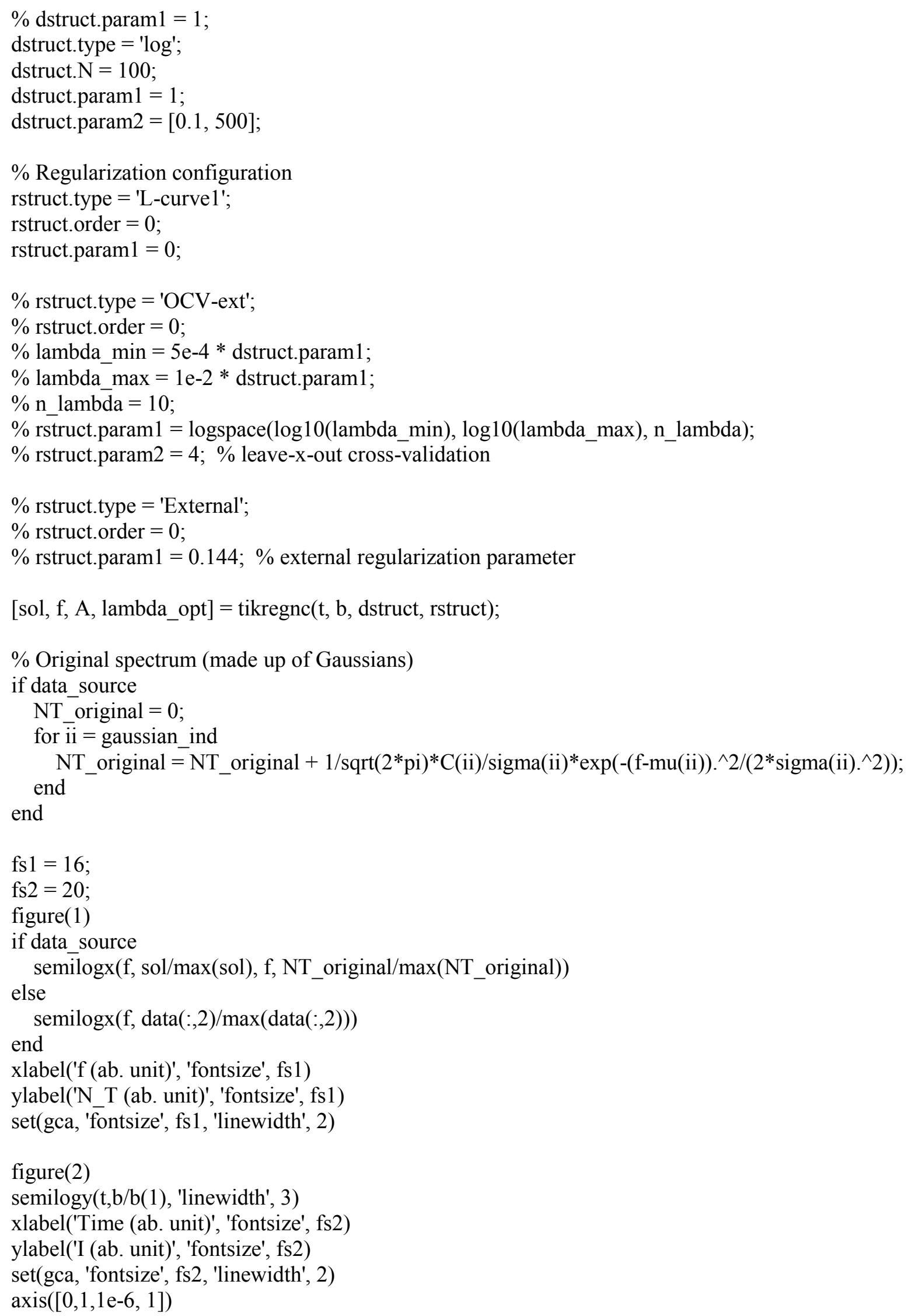




\section{tikregnc.m:}

function varargout $=$ tikregnc $(t, b$, dconfig, rconfig $)$

$\%$ A rewritten solver of Tikhonov regularization with non-negativity constraint

$\%$ Usage: $[\mathrm{X}]=$ tikregnc $(\mathrm{t}, \mathrm{b}$, dstruc, rconfig)

$\% \quad[\mathrm{X}, \mathrm{f}]=$ tikregnc $(\mathrm{t}, \mathrm{b}, \mathrm{dstruc}$, rconfig)

$\% \quad[\mathrm{X}, \mathrm{f}, \mathrm{A}, \mathrm{res}, \mathrm{smn}]=$ tikregnc $(\mathrm{t}, \mathrm{b}, \mathrm{dstruc}$, rconfig $)$

$\%$ Input arguments:

$\%$ t: time axis, a column vector

$\%$ b: observed data, a column vector

$\%$ dconfig: discretization configuration, a struct

$\% \quad$ dconfig.type: 'linear', 'log' or 'glq'

$\% \quad$ dconfig.N: discretization points in $\mathrm{f}$ space

$\%$ dconfig.param 1: scaling factor (a scalar). A few words about

$\% \quad$ scaling -- scaling is done for A matrix during the calculation

$\%$ for the purpose of adjusting the scale of regularization parameter

$\%$ because different value of regularization parameter tends to

$\% \quad$ give very different computing time. If A becauses $\mathrm{A} *$ sf, then

$\% \quad$ the corresponding soultion $\mathrm{x}$ becomes $\mathrm{x} / \mathrm{sf}$, and regularization

$\%$ paramter will be sf times larger (since it is actually lambda^ 2

$\% \quad$ before the $\|x\|_{2} 2^{\wedge} 2$ term.

$\% \quad$ update: seems like the scaling isn't really helping speed up

$\%$ the program

$\%$ dconfig.param2: [lower_bound, upper_bound] for 'linear' or 'log' type

$\%$ rconfig: regularization configuration, a struct

$\%$ rconfig.type: model selection type

$\% \quad \mathrm{X}$ : regularized solutions

$\%$ f_out: discretized f space

$\%$ A: discretization matrix

$\%$ lambda out: regularization parameter used in the end

$\%$

\% Written by Lijun Li, Oct, 2013

$\%$ 
$\%$ Update log:

$\%$ Mar, 2014: Add ordinary cross-validation for model selection

glq_alpha_ratio_tol $=1 \mathrm{e}-4 ; \%$ The tolerence of searching for the alpha value in 'glq'

$\mathrm{t}=$ force_column_vector $(\mathrm{t})$;

$\mathrm{b}=$ force_column_vector $(\mathrm{b})$;

$\left[\mathrm{A}, \mathrm{f} \_\right.$orig $]=$get_A(t, dconfig.type, dconfig. $\mathrm{N}$, dconfig.param2);

$\%$ note that $\mathrm{f}$ _orig is the real $\mathrm{f}$ grid for 'log' scaling, not $\log (\mathrm{f})$ grid

sf $=$ dconfig.param $1 ; \%$ scaling factor

$\mathrm{A}=\mathrm{A} * \mathrm{sf}$

$\%$ Solve the problem

L = full(get_l(length(f_orig), rconfig.order));

if strcmpi(rconfig.type, 'External')

$\%$ Use the externally provided regularization parameter

sol_scaled $=$ ncsolve $(A, b$, rconfig.param 1, L $)$;

lambda_opt $=$ rconfig.param $1 ; \%$ for the completeness of the output args

elseif strcmpi(rconfig.type, 'Morozov')

$\%$ Use Morozov discrepancy principle to determine the regularization

$\%$ parameter

delta $b=$ rconfig.param $1 ; \%$ noise amplitude of observed data $b$

lambda_opt=fsolve(@(lambda) lambda_func(lambda, A, b, L, delta_b), 1);

$[\mathrm{sol}]=$ ncsolve $(\mathrm{A}, \mathrm{b}$, lambda_opt, L);

elseif strcmpi(rconfig.type, 'OCV-ext')

$\%$ Ordinary cross validation with regularization parameters externally

$\%$ provided

$\%$ Note that the calling times of 11 _ls sove is length(lambda_arr) $* \mathrm{k} \_$fold

lambda_arr = rconfig.param $1 ; \%$ regularization parameter array provided externally

n_testdata $=$ rconfig.param $2 ; \%$ the number of test data points, or the data point of each subsample

$\mathrm{k} \_$fold $=$length(b) /n_testdata; $\% \mathrm{k}$ for $\mathrm{k}$-fold ordinary cross-validation

ocv_error_mean $=$ zeros $($ length(lambda_arr $), 1)$;

ocv_error_std $=$ zeros(length (lambda_arr), 1);

ocv_error_iter $=$ zeros $\left(\mathrm{k} \_\right.$fold, 1$) ; \%$ OCV error in each iteration below

$\operatorname{disp}([$ 'Going to do ', num2str(k_fold), '-fold cross-validation for ', num2str(length(lambda_arr)), ' regularization parameters'])

$\operatorname{disp}([$ 'Data points in each sub-sample $=$ ', num2str(n_testdata) $]$ )

$\operatorname{disp}([$ 'Discretization scaling factor $=$ ', num2str(dconfig.param1)] $)$

disp('----------------------------')

for $\mathrm{ii}=1$ :length(lambda_arr)

lambda_iter = lambda_arr(ii);

disp(['Iteration \#', num2str(ii), ', lambda = ', num2str(lambda_iter)])

for $\mathrm{jj}=1$ :k_fold 
$\%$ First compute the indices of training data and testing data ind_testdata $=$ zeros(length(n_testdata), 1);

for $\bar{k} \mathrm{k}=1: \mathrm{n}$ _testdata

end

ind_testdata $(\mathrm{kk})=\mathrm{jj}+\mathrm{k} \_$fold $*(\mathrm{kk}-1)$;

ind_whole $=1:$ length $(b)$;

ind_traindata $=$ ind_whole;

ind_traindata(ind_testdata) $=[] ; \%$ the way to get complementary data set from an array

$\%$ Now extract the training and testing data sets

b_test $=b$ (ind_testdata);

$\mathrm{t}$ test $=\mathrm{t}($ ind_testdata $)$;

b_train $=$ b(ind_traindata);

$\mathrm{t} \_$train $=\mathrm{t}($ ind_traindata $)$;

$\%$ Note the bumbers of columns of A_train and A_test are still the same

[A_train, f_train_orig] = get_A(t_train, dconfig.type, dconfig.N, dconfig.param2);

[A_test, f_test_orig] = get_A(t_test, dconfig.type, dconfig.N, dconfig.param2);

A train $=$ A train*sf;

A_test $=$ A_test*sf;

$\%$ Now solve the model based on training data solution

$x_{-}$train $=$ncsolve (A_train, b_train, lambda_iter, L); \% note that this is the scaled intermittent

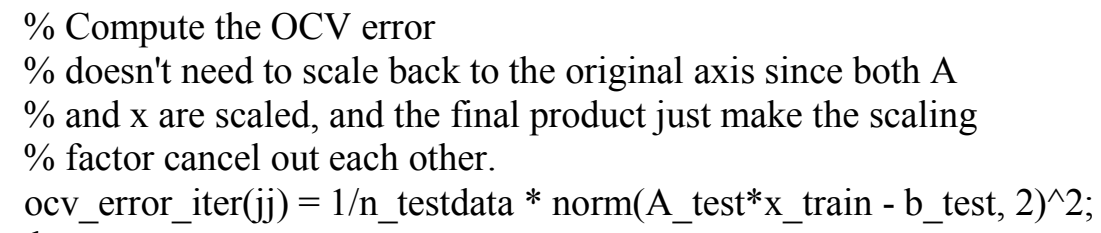

$\%$ Now compute the mean and variance of OCV error for this lambda_iter ocv_error_mean(ii) = mean (ocv_error_iter);

ocv_error_std(ii) $=$ std(ocv_error_iter);

disp $([$ 'OCV error mean = ', num2str(ocv_error_mean(ii)), ', OCV error standard deviation =', num2str(ocv_error_std(ii))])

$\operatorname{disp}(' \mathrm{OC} \mathrm{V}$ error for this iteration: ')

disp(ocv_error_iter)

end

$\%$ Save the lambda path data

data_out $=$ [force_column_vector(lambda_arr), ocv_error_mean, ocv_error_std];

save('-ascii', 'ocv_lambda_path.dat', 'data_out')

disp('Lambda path data saved to "ocv_lambda_path.dat".')

$\%$ Now determine the optimal lambda based on those results

$\%$ Results are also plotted in this function

lambda_opt $=$ find_lambda_opt_ocv(lambda_arr, ocv_error_mean, ocv_error_std); 
$\%$ Compute the final solution

sol_scaled $=\operatorname{ncsolve}(\mathrm{A}, \mathrm{b}$, lambda_opt, L);

elseif strcmpi(rconfig.type, 'L-curve1')

$\%$ Use L-curve criterion to determine the regularization parameter

1_curve_plot $=$ rconfig.param1;

$[\mathrm{U}, \mathrm{sm}, \mathrm{XX}, \mathrm{V}]=\operatorname{cgsvd}(\mathrm{A}, \mathrm{L}) ; \%$ Generalized singular value decomposition in compact form figure(1)

[lc0, rho, eta, reg_params $]=1$ _curve $(\mathrm{U}, \mathrm{sm}, \mathrm{b}$, 'Tikh');

$\% \mathrm{lc} 0$ is the regularization parameter from the unconstrained problem,

$\%$ which can be used as the initial value for the search of

$\%$ the regularization parameter for the constrained problem

close all

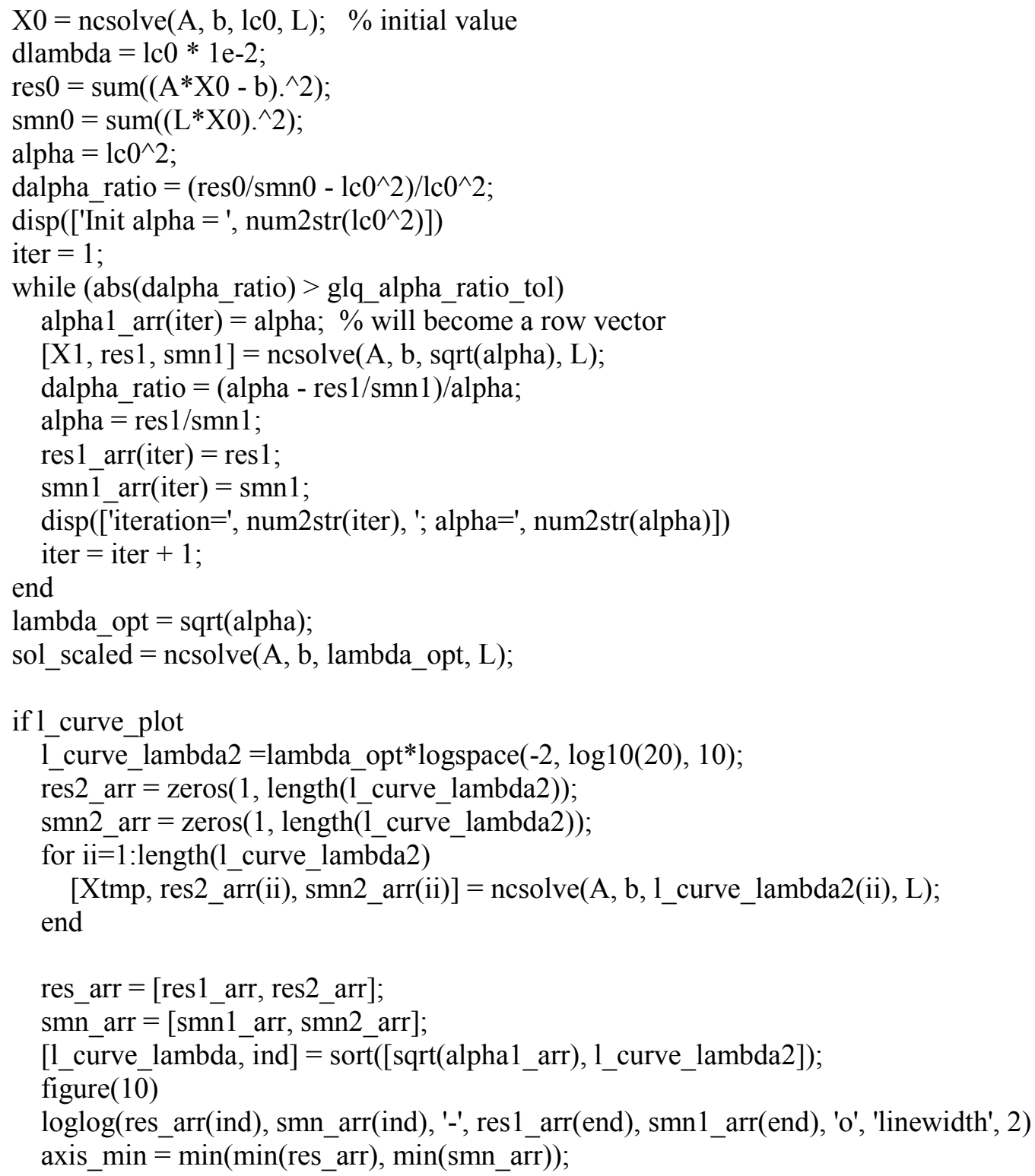




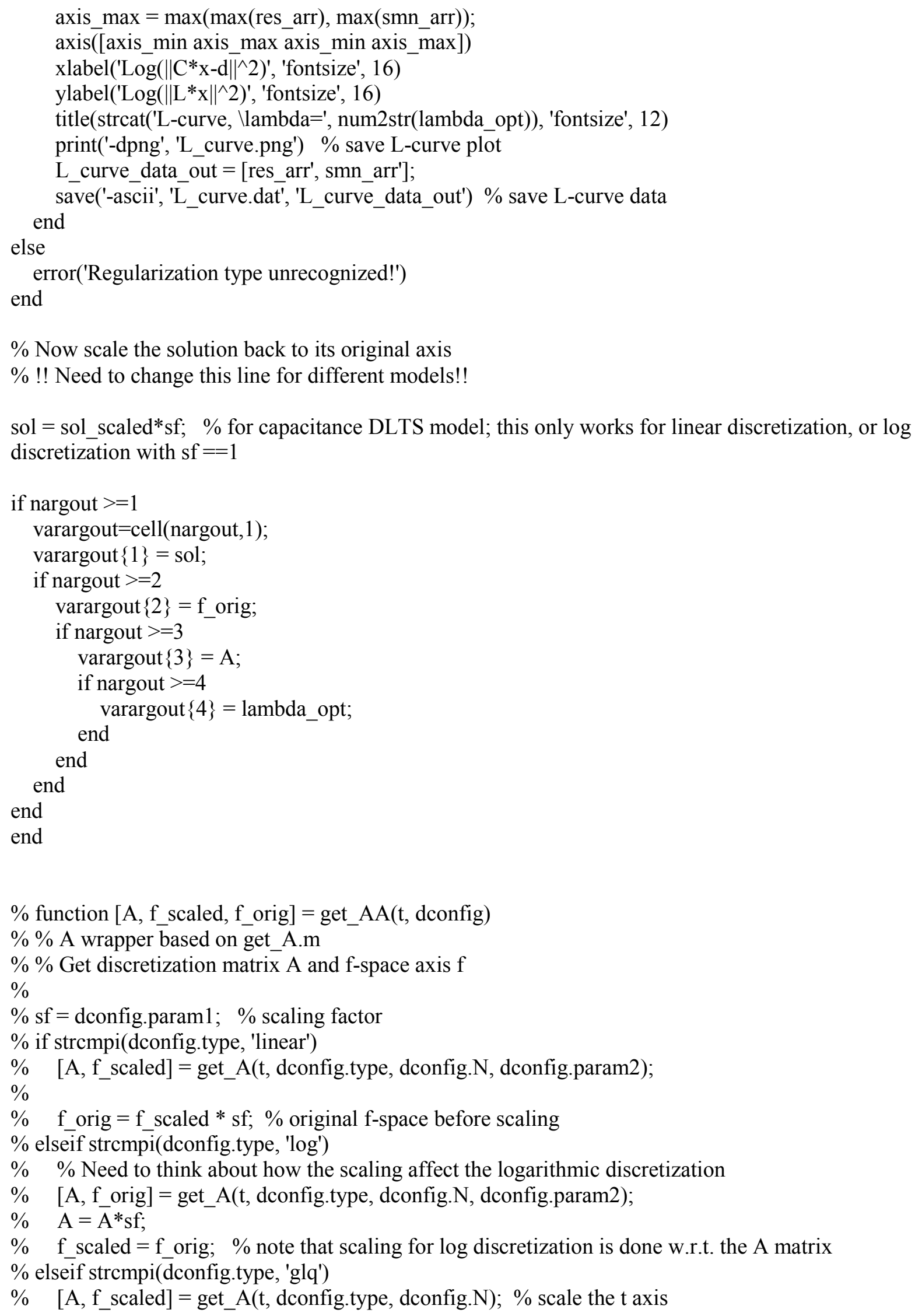


$\% \quad$ f_orig $=\mathrm{f}$ _scaled $*$ sf; $\%$ original $\mathrm{f}$-space before scaling

$\%$ end

$\% \% \mathrm{~A}=\operatorname{sparse}(\mathrm{A}) ; \% 11$ ls program can take the advantage of sparse matrix

$\%$

$\%$ end

function v_out $=$ force_column_vector(v_in)

$\%$ Convert the input to column vector if it is not

if $\sim$ iscolumn $\left(v_{-}\right.$in $)$

v_out = v_in';

else

v_out $=$ v_in;

end

end

function $\mathrm{y}=$ lambda_func(lambda, A, b, L, delta_b)

$\%$ The affiliated function when solving $\|\mathrm{A} * \mathrm{x}-\mathrm{b}\|=$ delta_b when using

$\%$ Morozov discrepancy principle to select regularization parameters

$\%$

$\%$ Input args:

$\%$ lambda: regularization parameter

$\% \mathrm{~A}, \mathrm{~b}, \mathrm{~L}$ : same as those defined in tikregnc.m

$\%$ delta b: noise amplitude in b, a scalar

$\%$

$\mathrm{x}=$ ncsolve $(\mathrm{A}, \mathrm{b}, \mathrm{lambda}, \mathrm{L})$;

$\mathrm{y}=\left(\mathrm{A}^{*} \mathrm{x}-\mathrm{b}\right)^{\prime *}\left(\mathrm{~A}^{*} \mathrm{x}-\mathrm{b}\right)-$ delta_ $\mathrm{b}^{\wedge} 2$;

end

\section{ncsolve.m}

function varargout $=$ ncsolve $(A, b$, lambda, $\mathrm{L})$

$\%$ Examples:

$\% \quad \mathrm{X}=$ ncsolve $(\mathrm{A}, \mathrm{b}$, lambda, $\mathrm{L})$;

$\% \quad[\mathrm{X}, \mathrm{res}, \mathrm{smn}]=\mathrm{ncsolve}(\mathrm{A}, \mathrm{b}$, lambda, $\mathrm{L})$;

$\%$ Solve the linear least square program with $\mathrm{x}>=0$ constraint

$\% \min \left\|\mathrm{C}^{*} \mathrm{x}-\mathrm{d}\right\|$ subject to $\mathrm{A}^{*} \mathrm{x}<=\mathrm{b}, \mathrm{A} \_$eq* $\mathrm{x}=\mathrm{b} \_$eq, $\mathrm{lb}<=\mathrm{x}<=\mathrm{ub}$

$\%$ Input args:

$\% \quad$ A: Discretization matrix

$\% \quad$ b: data (the transient current)

$\%$ lambda: the regularization parameter

$\% \quad$ L: derivative matrix

$\%$ (Optional) output args:

$\% \quad \mathrm{X}$ : the solution

$\%$ rmn: fitting residue

$\%$ smn: smoothness

$\mathrm{x} 0=\operatorname{zeros}(\operatorname{size}(\mathrm{L}, 2), 1) ; \%$ default

$\mathrm{N}=\operatorname{size}(\mathrm{A}, 2)$;

$\mathrm{C}=[\mathrm{A} ;$ lambda*L $]$

$\mathrm{d}=[\mathrm{b} ;$ lambda* L*x0];

$\mathrm{AA}=-\operatorname{eye}(\mathrm{N}, \mathrm{N})$; 


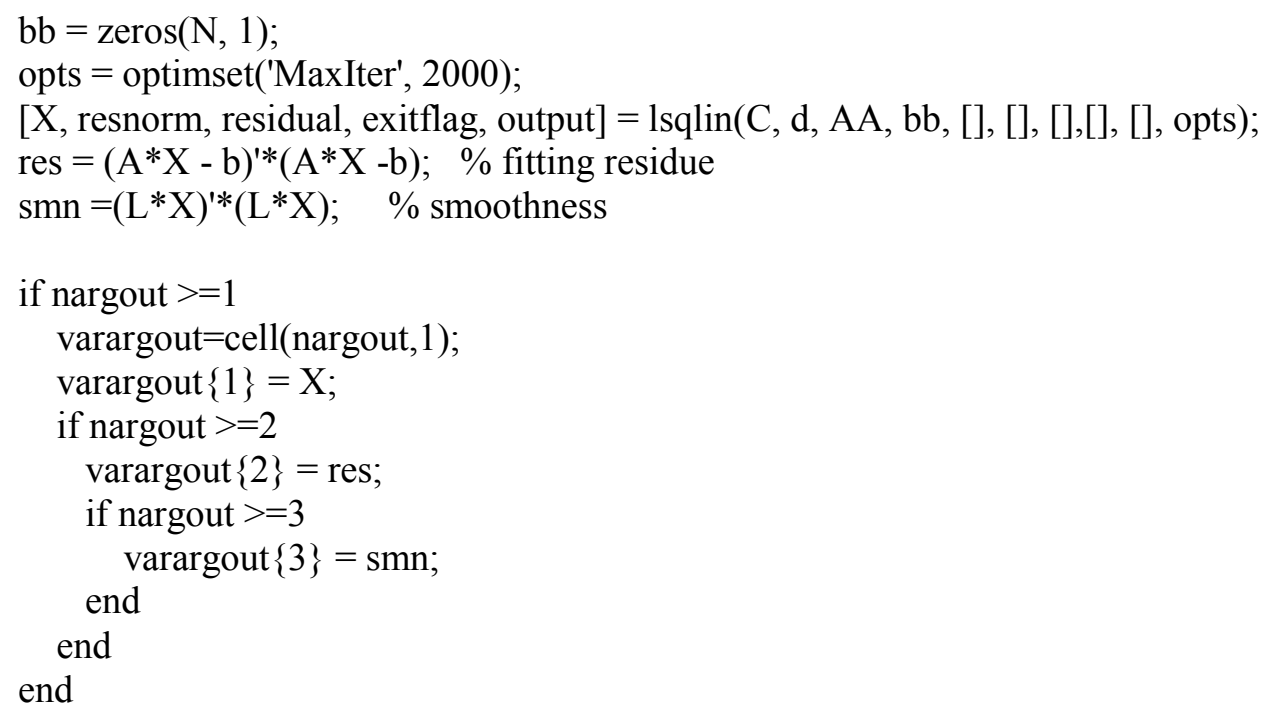




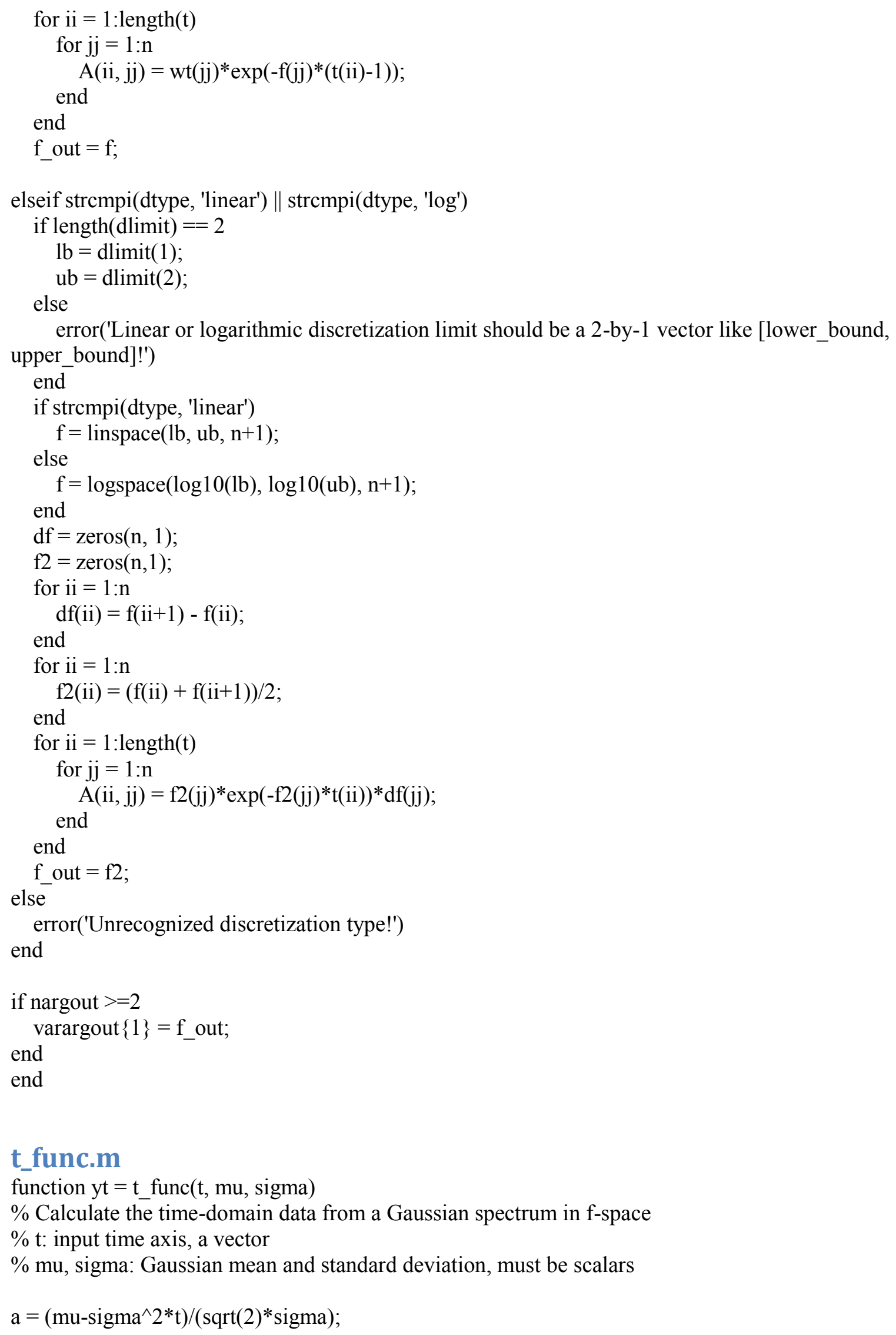

t_func.m

function $\mathrm{yt}=\mathrm{t}$ func $(\mathrm{t}, \mathrm{mu}$, sigma $)$

$\%$ Calculate the time-domain data from a Gaussian spectrum in f-space

$\% \mathrm{t}$ : input time axis, a vector

$\%$ mu, sigma: Gaussian mean and standard deviation, must be scalars

$\mathrm{a}=\left(\right.$ mu-sigma $\left.{ }^{\wedge} 2 * \mathrm{t}\right) /(\operatorname{sqrt}(2) * \operatorname{sigma})$ 
$\mathrm{A}=\exp (-\mathrm{mu} * \mathrm{t}+($ sigma*t).^2/2);

$\mathrm{B}=\left(\exp \left(-\mathrm{a} .{ }^{\wedge} 2\right)+\operatorname{sqrt}(\mathrm{pi}) * \mathrm{a} \cdot *(1+\operatorname{erf}(\mathrm{a}))\right)$

$\mathrm{yt}=\operatorname{sigma} / \operatorname{sqrt}(2 * \mathrm{pi}) * \mathrm{~A} . * \mathrm{~B}$;

end

\section{gen_laguerre_rulle2.m}

function $[\mathrm{x}, \mathrm{w}]=$ gen_laguerre rule2 (order, alpha, $\mathrm{a}, \mathrm{b})$

$\%[\mathrm{x}, \mathrm{w}]=$ gen_laguerre_rule2 (order, alpha, $\mathrm{a}, \mathrm{b})$

$\%$ This script is modified from

http://people.sc.fsu.edu/ jburkardt/m_src/gen_laguerre_rule/gen_laguerre_rule.html

$\%$

$\%$ This program computes a standard or exponentially weighted generalized Gauss-Laguerre quadrature rule.

$\%$ Integral $(0<=x<$ oo $)|x-a|^{\wedge} A L P H A \exp \left(-B^{*}(x-a)\right) f(x) d x$

$\%$ Input args:

$\%$ order: number of points in the rule

$\%$ alpha: the exponent of $|X|$

$\%$ a: the left endpoint of the interval of integration, and is currently fixed at 0

$\%$ b: the scale factor in the exponential

$\%$

\% Modified: 24 February 2010; Author: John Burkardt

$\%$ Further modified by Lijun Li (add output args $[\mathrm{x}, \mathrm{w}]$, reduce the verbose output, remove filename option)

\% Licensing: This code is distributed under the GNU LGPL license.

$\%$ Initialize the parameters.

beta $=0.0$

$\%$ Construct the rule.

kind $=5$;

$[\mathrm{x}, \mathrm{w}]=\operatorname{cgqf}($ order, kind, alpha, beta, $\mathrm{a}, \mathrm{b})$;

end

function [ $\mathrm{t}$, wts ] $=\operatorname{cdgqf}(\mathrm{nt}, \mathrm{kind}$, alpha, beta $)$

$\% \%$ CDGQF computes a Gauss quadrature formula with default A, B and simple knots.

$\%$

$\%$ Discussion:

$\%$

$\%$ This routine computes all the knots and weights of a Gauss quadrature

$\%$ formula with a classical weight function with default values for $\mathrm{A}$ and $\mathrm{B}$,

$\%$ and only simple knots.

$\%$ There are no moments checks and no printing is done.

$\%$ Use routine EIQFS to evaluate a quadrature computed by CGQFS.

$\%$

$\%$ Licensing: This code is distributed under the GNU LGPL license.

$\%$ Modified: 04 January 2010

$\%$ Original FORTRAN77 version by Sylvan Elhay, Jaroslav Kautsky.

$\%$ MATLAB version by John Burkardt.

$\%$ 
$\%$ Reference:

$\%$ Sylvan Elhay, Jaroslav Kautsky,

$\%$ Algorithm 655: IQPACK, FORTRAN Subroutines for the Weights of

$\%$ Interpolatory Quadrature,

$\%$ ACM Transactions on Mathematical Software,

$\%$ Volume 13, Number 4, December 1987, pages 399-415.

$\%$

$\%$ Parameters:

$\%$ Input, integer NT, the number of knots.

$\%$ Input, integer KIND, the rule.

$\% \quad$ 1, Legendre, $\quad(\mathrm{a}, \mathrm{b}) \quad 1.0$

$\% \quad 2$, Chebyshev, $\quad(a, b) \quad((b-x) *(x-a))^{\wedge}(-0.5)$

$\% \quad 3$, Gegenbauer, $\quad(a, b) \quad\left((b-x)^{*}(x-a)\right)^{\wedge}$ alpha

$\% \quad$ 4, Jacobi, $\quad(a, b) \quad(b-x)^{\wedge}$ alpha* $(x-a)^{\wedge}$ beta

$\% \quad$ 5, Generalized Laguerre, (a,inf) $\quad(x-a)^{\wedge} a l p h a * \exp \left(-b^{*}(x-a)\right)$

$\% \quad 6$, Generalized Hermite, (-inf,inf) $|x-a|^{\wedge}$ alpha*exp(-b*(x-a)^2)

$\% \quad 7$, Exponential, $\quad(\mathrm{a}, \mathrm{b}) \quad|\mathrm{x}-(\mathrm{a}+\mathrm{b}) / 2.0|^{\wedge}$ alpha

$\% \quad 8$, Rational, $\quad$ (a,inf) $\quad(\mathrm{x}-\mathrm{a})^{\wedge}$ alpha* $(\mathrm{x}+\mathrm{b})^{\wedge}$ beta

$\%$

$\%$ Input, real ALPHA, the value of Alpha, if needed.

$\%$ Input, real BETA, the value of Beta, if needed.

$\%$ Output, real T(NT), the knots.

$\%$ Output, real WTS(NT), the weights.

$\%$

parchk ( kind, $2 *$ nt, alpha, beta );

$\%$

$\%$ Get the Jacobi matrix and zero-th moment.

$[\mathrm{aj}, \mathrm{bj}$, zemu $]=$ class_matrix $($ kind, nt, alpha, beta $)$; $\%$

$\%$ Compute the knots and weights.

$[\mathrm{t}, \mathrm{wts}]=\operatorname{sgqf}(\mathrm{nt}, \mathrm{aj}, \mathrm{bj}, \mathrm{zemu})$;

return

end

function $[\mathrm{t}, \mathrm{wts}]=\operatorname{cgqf}(\mathrm{nt}$, kind, alpha, beta, a, b )

$\% \%$ CGQF computes knots and weights of a Gauss quadrature formula.

$\%$

$\%$ Discussion:

$\%$ The user may specify the interval (A,B).

$\%$ Only simple knots are produced.

$\%$ The user may request that the routine print the knots and weights,

$\%$ and perform a moment check.

$\%$ Use routine EIQFS to evaluate this quadrature formula.

$\%$

$\%$ Licensing: This code is distributed under the GNU LGPL license.

$\%$

$\%$ Modified: 16 February 2010

$\%$

$\% \quad$ Original FORTRAN77 version by Sylvan Elhay, Jaroslav Kautsky.

$\%$ MATLAB version by John Burkardt.

$\%$ 
$\%$ Reference:

$\%$ Sylvan Elhay, Jaroslav Kautsky,

$\%$ Algorithm 655: IQPACK, FORTRAN Subroutines for the Weights of

$\%$ Interpolatory Quadrature,

$\%$ ACM Transactions on Mathematical Software,

$\%$ Volume 13, Number 4, December 1987, pages 399-415.

$\%$

$\%$ Parameters:

$\%$ Input, integer NT, the number of knots.

$\%$ Input, integer KIND, the rule.

$\% \quad 1$, Legendre, $\quad(a, b) \quad 1.0$

$\% \quad$ 2, Chebyshev Type 1, (a,b) $\quad\left((\mathrm{b}-\mathrm{x})^{*}(\mathrm{x}-\mathrm{a})\right)^{\wedge}(-0.5)$

$\%$ 3, Gegenbauer,

$\%$ 4, Jacobi, $(a, b) \quad(b-x)^{\wedge}$ alpha* $(x-a)^{\wedge}$ beta

$\% \quad$ 5, Generalized Laguerre, $(a,+o o) \quad(x-a)^{\wedge}$ alpha* $\exp \left(-b^{*}(x-a)\right)$

$\% \quad 6$, Generalized Hermite, $(-0 o,+\infty) \quad|x-a|^{\wedge}$ alpha* $\exp \left(-b^{*}(x-a)^{\wedge} 2\right)$

$\% \quad 7$, Exponential, $\quad(\mathrm{a}, \mathrm{b}) \quad|\mathrm{x}-(\mathrm{a}+\mathrm{b}) / 2.0|^{\wedge}$ alpha

$\% \quad 8$, Rational, $\quad(\mathrm{a},+\mathrm{oo}) \quad(\mathrm{x}-\mathrm{a})^{\wedge}$ alpha* $(\mathrm{x}+\mathrm{b})^{\wedge}$ beta

$\% \quad 9$, Chebyshev Type 2, (a,b) $\quad\left((\mathrm{b}-\mathrm{x})^{*}(\mathrm{x}-\mathrm{a})\right)^{\wedge}(+0.5)$

$\%$

$\%$ Input, real ALPHA, the value of Alpha, if needed.

$\%$ Input, real BETA, the value of Beta, if needed.

$\%$ Input, real A, B, the interval endpoints.

$\%$ Output, real T(NT), the knots.

$\%$ Output, real WTS(NT), the weights.

$\%$

$\%$ Compute the Gauss quadrature formula for default values of A and B.

[ $\mathrm{t}, \mathrm{wts}]=\operatorname{cdgqf}(\mathrm{nt}, \mathrm{kind}$, alpha, beta $)$;

$\%$ All knots have multiplicity $=1$.

$\mathrm{mlt}=\operatorname{zeros}(\mathrm{nt}, 1)$;

$\operatorname{mlt}(1: \mathrm{nt})=1$

$\%$

$\% \operatorname{NDX}(\mathrm{I})=\mathrm{I}$.

$\%$

$\mathrm{ndx}=(1: \mathrm{nt})$

$\%$

$\%$ Scale the quadrature rule.

[ $\mathrm{t}$, wts ] $=\operatorname{scqf}(\mathrm{nt}, \mathrm{t}, \mathrm{mlt}$, wts, nt, ndx, kind, alpha, beta, a, b ); return

end

function [ aj, bj, zemu ] = class_matrix ( kind, m, alpha, beta )

$\% \%$ CLASS_MATRIX computes the Jacobi matrix for a quadrature rule.

$\%$ Discussion:

$\% \quad$ This routine computes the diagonal AJ and subdiagonal BJ

$\%$ elements of the order $\mathrm{M}$ tridiagonal symmetric Jacobi matrix

$\%$ associated with the polynomials orthogonal with respect to

$\%$ the weight function specified by KIND.

$\%$

$\%$ For weight functions 1-7, $\mathrm{M}$ elements are defined in BJ even

$\%$ though only M-1 are needed. For weight function $8, \mathrm{BJ}(\mathrm{M})$ is

$\%$ set to zero. 
$\%$ The zero-th moment of the weight function is returned in ZEMU.

$\%$ Licensing: This code is distributed under the GNU LGPL license.

$\%$

\% Modified: 04 January 2010

$\%$

$\% \quad$ Original FORTRAN77 version by Sylvan Elhay, Jaroslav Kautsky.

$\%$ MATLAB version by John Burkardt.

$\%$

$\%$ Reference:

$\%$ Sylvan Elhay, Jaroslav Kautsky,

$\%$ Algorithm 655: IQPACK, FORTRAN Subroutines for the Weights of

$\%$ Interpolatory Quadrature,

$\%$ ACM Transactions on Mathematical Software,

$\%$ Volume 13, Number 4, December 1987, pages 399-415.

$\%$

$\%$ Parameters:

$\%$ Input, integer KIND, the rule.

$\% \quad 1$, Legendre, $\quad(\mathrm{a}, \mathrm{b}) \quad 1.0$

$\% \quad 2$, Chebyshev, $\quad(a, b) \quad((b-x) *(x-a))^{\wedge}(-0.5)$

$\% \quad 3$, Gegenbauer, $\quad(a, b) \quad((b-x) *(x-a))^{\wedge}$ alpha

$\% \quad$ 4, Jacobi, $\quad(a, b) \quad(b-x)^{\wedge}$ alpha* $(x-a)^{\wedge}$ beta

$\% \quad$ 5, Generalized Laguerre, $(a$, inf $) \quad(x-a)^{\wedge}$ alpha*exp $(-b *(x-a))$

$\% \quad 6$, Generalized Hermite, (-inf,inf) $|x-a|^{\wedge}$ alpha*exp $\left(-b *(x-a)^{\wedge} 2\right)$

$\% \quad 7$, Exponential, $\quad(\mathrm{a}, \mathrm{b}) \quad|\mathrm{x}-(\mathrm{a}+\mathrm{b}) / 2.0|^{\wedge}$ alpha

$\%$ 8, Rational, $\quad(\mathrm{a}$, inf $) \quad(\mathrm{x}-\mathrm{a})^{\wedge}$ alpha* $(\mathrm{x}+\mathrm{b})^{\wedge}$ beta

$\%$

$\%$ Input, integer $\mathrm{M}$, the order of the Jacobi matrix.

$\%$ Input, real ALPHA, the value of Alpha, if needed.

$\%$ Input, real BETA, the value of Beta, if needed.

$\%$ Output, real $\mathrm{AJ}(\mathrm{M}), \mathrm{BJ}(\mathrm{M})$, the diagonal and subdiagonal

$\%$ of the Jacobi matrix.

$\%$ Output, real ZEMU, the zero-th moment.

$\%$

temp = eps;

parchk ( kind, $2 * \mathrm{~m}-1$, alpha, beta );

temp2 $=0.5$;

if $\left(500.0 *\right.$ temp $<$ abs $\left((\text { gamma }(\text { temp2 }))^{\wedge} 2-\right.$ pi $\left.)\right)$

fprintf ( 1, ' $\mathrm{n}$ ' );

fprintf ( 1 , 'CLASS - Fatal error! $\backslash n ')$;

fprintf ( 1 , ' Gamma function does not match machine parameters. $\backslash n '$ ');

error ( 'CLASS - Fatal error!' );

end

$\mathrm{bj}=\mathrm{zeros}(\mathrm{m}, 1)$;

$a j=\operatorname{zeros}(m, 1)$;

if $($ kind $==1)$

$\mathrm{ab}=0.0$;

zemu $=2.0 /(\mathrm{ab}+1.0)$;

$\operatorname{aj}(1: m)=0.0$; 


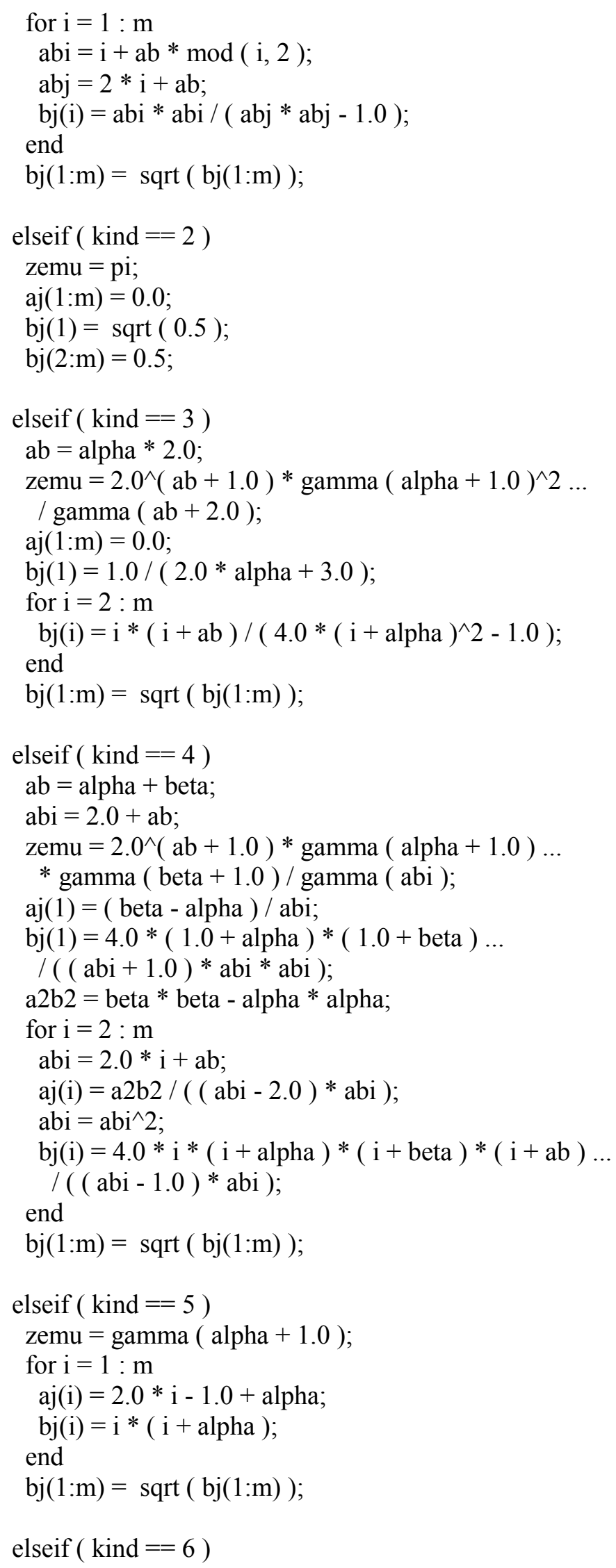




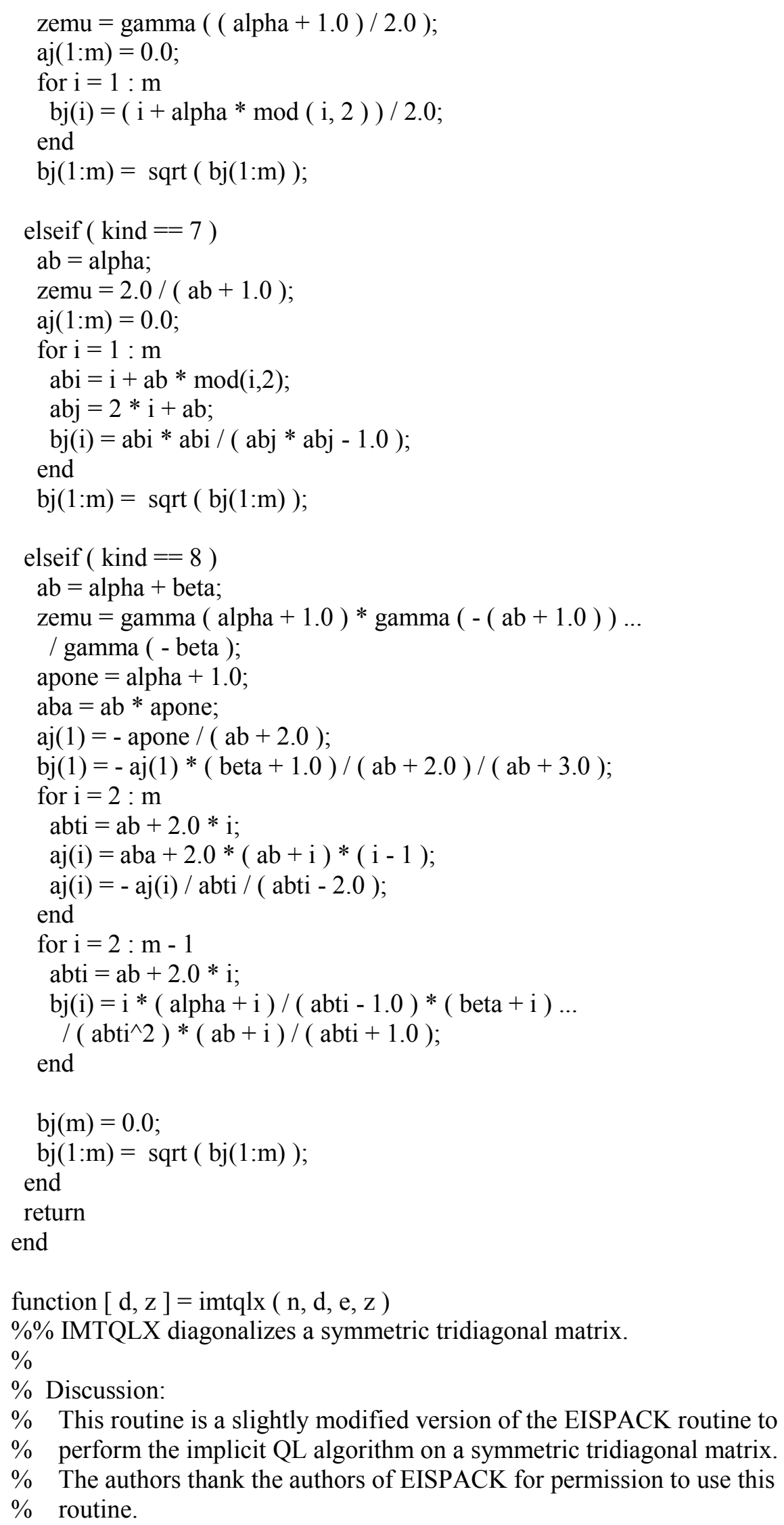


$\% \quad$ It has been modified to produce the product $\mathrm{Q}^{\prime} * \mathrm{Z}$, where $\mathrm{Z}$ is an input

$\%$ vector and $\mathrm{Q}$ is the orthogonal matrix diagonalizing the input matrix.

$\%$ The changes consist (essentialy) of applying the orthogonal transformations

$\%$ directly to $\mathrm{Z}$ as they are generated.

$\%$ Licensing: This code is distributed under the GNU LGPL license.

$\%$

$\%$ Modified: 04 January 2010

$\%$ Original FORTRAN77 version by Sylvan Elhay, Jaroslav Kautsky.

$\% \quad$ MATLAB version by John Burkardt.

$\%$

$\%$ Reference:

$\%$ Sylvan Elhay, Jaroslav Kautsky,

$\%$ Algorithm 655: IQPACK, FORTRAN Subroutines for the Weights of

$\%$ Interpolatory Quadrature,

$\%$ ACM Transactions on Mathematical Software,

$\%$ Volume 13, Number 4, December 1987, pages 399-415.

$\%$

$\%$ Roger Martin, James Wilkinson,

$\%$ The Implicit QL Algorithm,

$\%$ Numerische Mathematik,

$\%$ Volume 12, Number 5, December 1968, pages 377-383.

$\%$

$\%$ Parameters:

$\%$ Input, integer $\mathrm{N}$, the order of the matrix.

$\%$ Input, real $\mathrm{D}(\mathrm{N})$, the diagonal entries of the matrix.

$\%$ Input, real $\mathrm{E}(\mathrm{N})$, the subdiagonal entries of the

$\%$ matrix, in entries $\mathrm{E}(1)$ through $\mathrm{E}(\mathrm{N}-1)$.

$\%$ Input, real $\mathrm{Z}(\mathrm{N})$, a vector to be operated on.

$\%$ Output, real $\mathrm{D}(\mathrm{N})$, the diagonal entries of the diagonalized matrix.

$\%$ Output, real $\mathrm{Z}(\mathrm{N})$, the value of $\mathrm{Q}^{\prime} * \mathrm{Z}$, where $\mathrm{Q}$ is the matrix that

$\%$ diagonalizes the input symmetric tridiagonal matrix.

$\%$

itn $=30$;

prec $=$ eps;

if $(\mathrm{n}==1)$

return

end

$\mathrm{e}(\mathrm{n})=0.0$

for $1=1: \mathrm{n}$

$\mathrm{j}=0$;

while ( 1 )

for $\mathrm{m}=1: \mathrm{n}$

if $(\mathrm{m}==\mathrm{n})$

break

end

if $(\operatorname{abs}(\mathrm{e}(\mathrm{m}))<=\operatorname{prec} *(\operatorname{abs}(\mathrm{d}(\mathrm{m}))+\operatorname{abs}(\mathrm{d}(\mathrm{m}+1))))$

break

end 


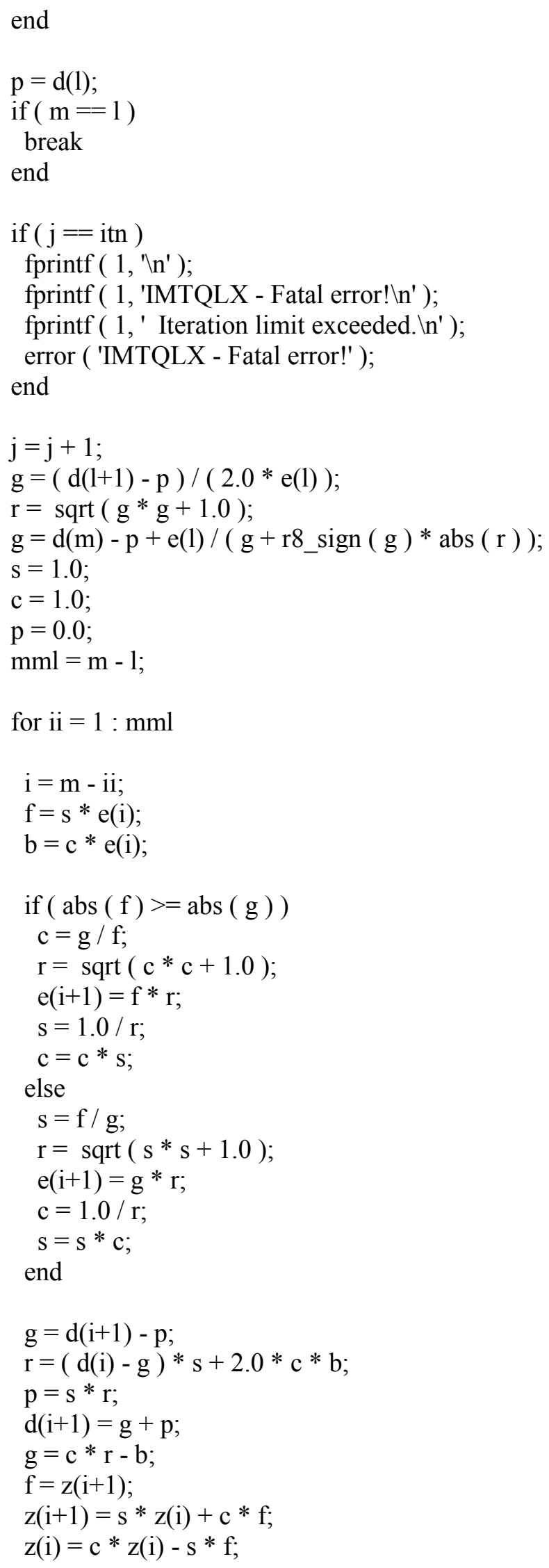




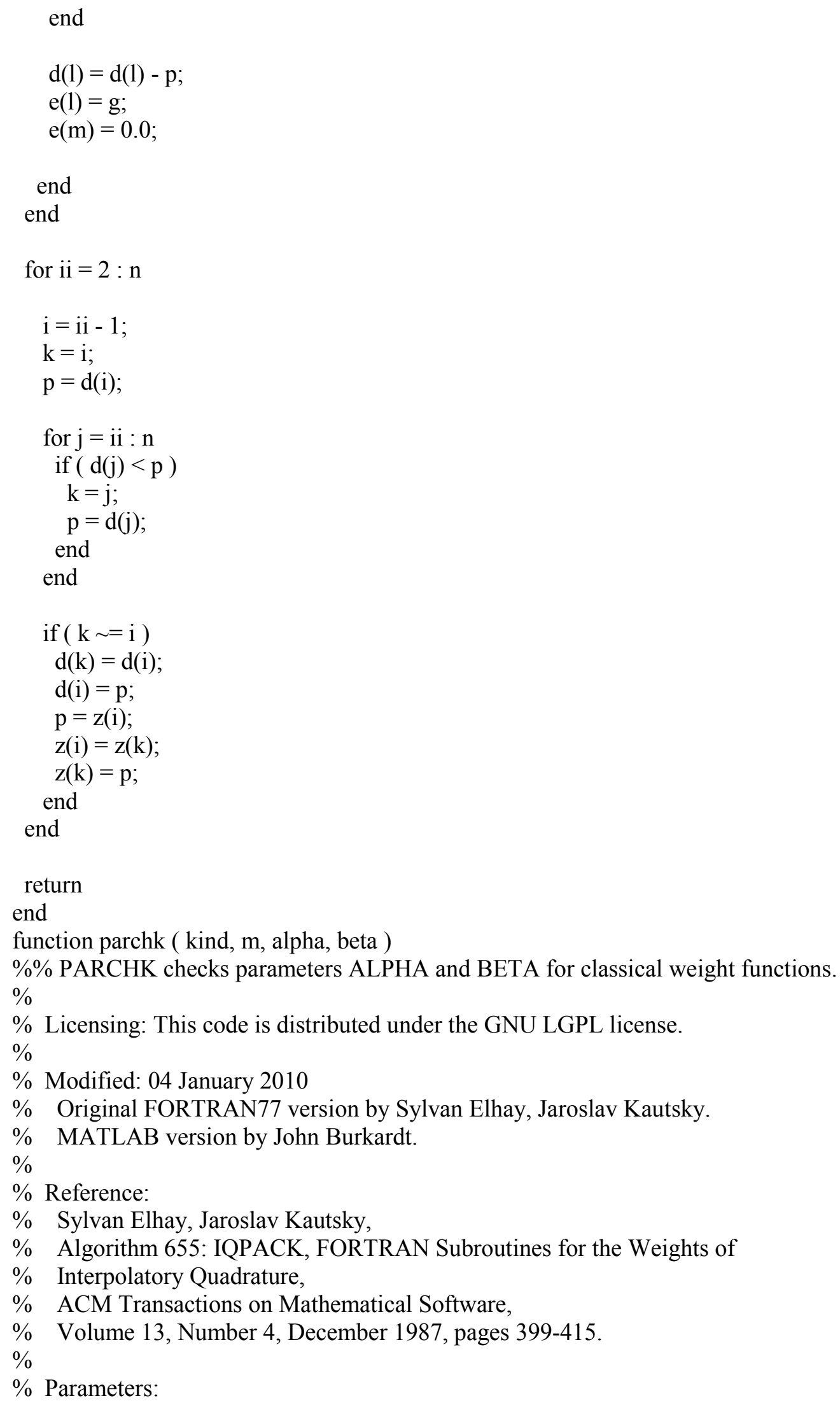


$\%$ Input, integer KIND, the rule.

$\% \quad 1$, Legendre, $\quad(\mathrm{a}, \mathrm{b}) \quad 1.0$

$\% \quad 2$, Chebyshev, $\quad(a, b) \quad((b-x) *(x-a))^{\wedge}(-0.5)$

$\% \quad 3$, Gegenbauer, $\quad(a, b) \quad\left((b-x)^{*}(x-a)\right)^{\wedge}$ alpha

$\% \quad$ 4, Jacobi, $\quad(a, b) \quad(b-x)^{\wedge}$ alpha* $(x-a)^{\wedge}$ beta

$\% \quad$ 5, Generalized Laguerre, (a,inf) $\quad(x-a)^{\wedge}$ alpha* $\exp \left(-b^{*}(x-a)\right)$

$\% \quad 6$, Generalized Hermite, (-inf,inf) $|\mathrm{x}-\mathrm{a}|^{\wedge}$ alpha* $\exp \left(-\mathrm{b}^{*}(\mathrm{x}-\mathrm{a})^{\wedge} 2\right)$

$\% \quad 7$, Exponential, $\quad(\mathrm{a}, \mathrm{b}) \quad|\mathrm{x}-(\mathrm{a}+\mathrm{b}) / 2.0|^{\wedge} \mathrm{alpha}$

$\% \quad 8$, Rational, $\quad(\mathrm{a}$, inf $) \quad(\mathrm{x}-\mathrm{a})^{\wedge}$ alpha* $(\mathrm{x}+\mathrm{b})^{\wedge}$ beta

$\%$

$\%$ Input, integer $\mathrm{M}$, the order of the highest moment to

$\%$ be calculated. This value is only needed when $\mathrm{KIND}=8$.

$\%$

$\%$ Input, real ALPHA, BETA, the parameters, if required

$\%$ by the value of KIND.

$\%$

if $($ kind $<=0$ )

fprintf ( 1, ' $n$ ')

fprintf ( 1 , 'PARCHK - Fatal error! \n' );

fprintf ( $1,{ }^{\prime}$ KIND $<=0 . \ln$ ');

error ( 'PARCHK - Fatal error!' );

end

$\%$

$\%$ Check ALPHA for Gegenbauer, Jacobi, Laguerre, Hermite, Exponential.

$\%$

if $(3<=$ kind $\& \&$ alpha $<=-1.0)$

fprintf ( 1, ' 'n' );

fprintf ( 1 , 'PARCHK - Fatal error! $\backslash n '$ );

fprintf ( $1,{ }^{\prime} 3<=$ KIND and ALPHA $<=-1 . \ln$ ' );

error ( 'PARCHK - Fatal error!' );

end

$\%$

$\%$ Check BETA for Jacobi.

$\%$

if $($ kind $==4 \& \&$ beta $<=-1.0$ )

fprintf ( 1, ' 'n');

fprintf ( 1 , 'PARCHK - Fatal error! $\backslash n '$ );

fprintf ( $1,{ }^{\prime}$ KIND $==4$ and BETA $\left.<=-1.0 . \backslash n '\right)$;

error ( 'PARCHK - Fatal error!' );

end

$\%$

$\%$ Check ALPHA and BETA for rational.

$\%$

if $($ kind $==8)$

tmp $=$ alpha + beta $+\mathrm{m}+1.0$;

if $(0.0<=$ tmp $\|$ tmp $<=$ beta $)$

fprintf $(1$, ' 'n' );

fprintf ( 1, 'PARCHK - Fatal error! \n' );

fprintf ( $1,{ }^{\prime}$ KIND $==8$ but condition on ALPHA and BETA fails. $\left.\mid n^{\prime}\right)$;

error ( 'PARCHK - Fatal error!' );

end 


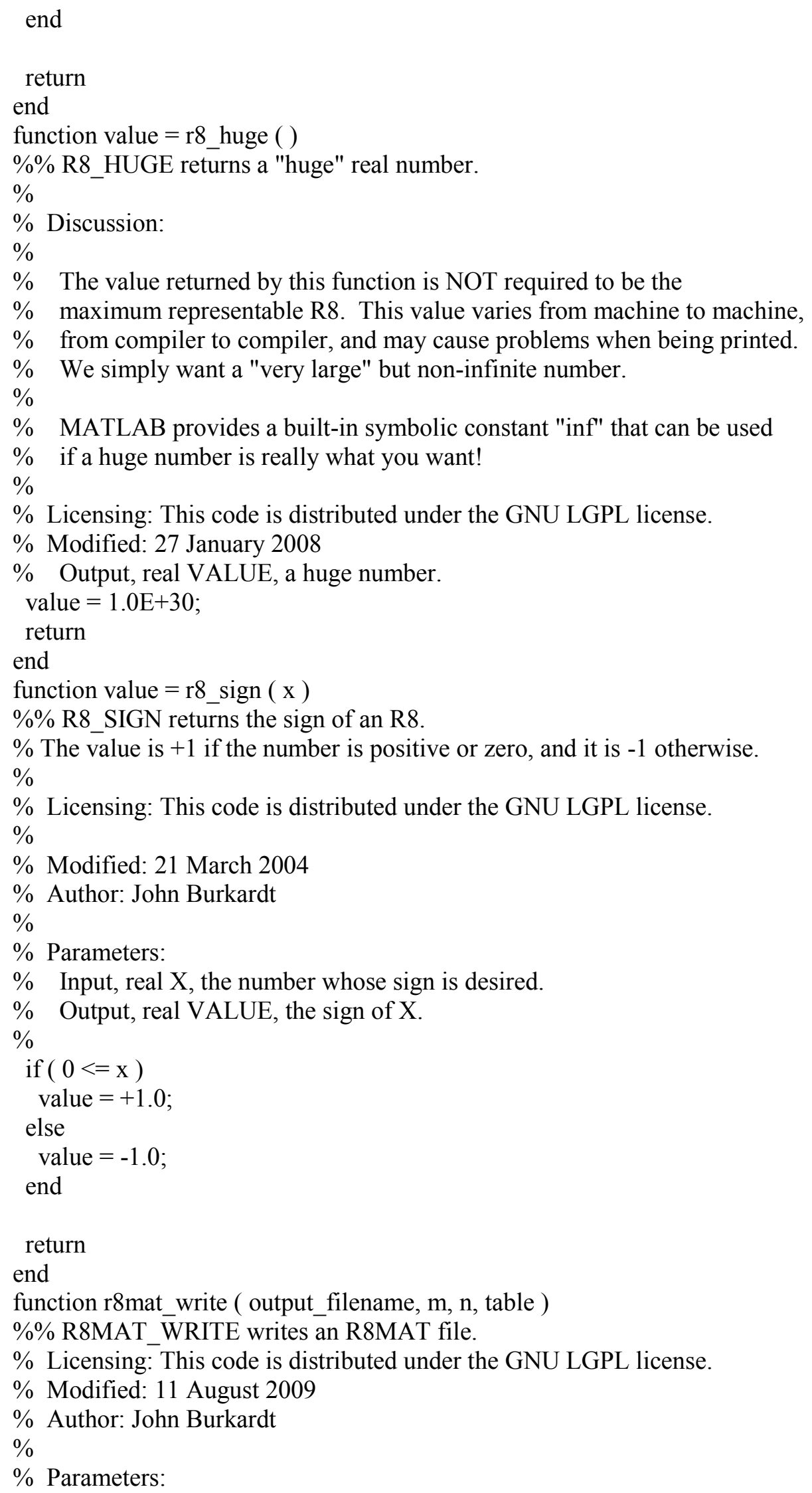




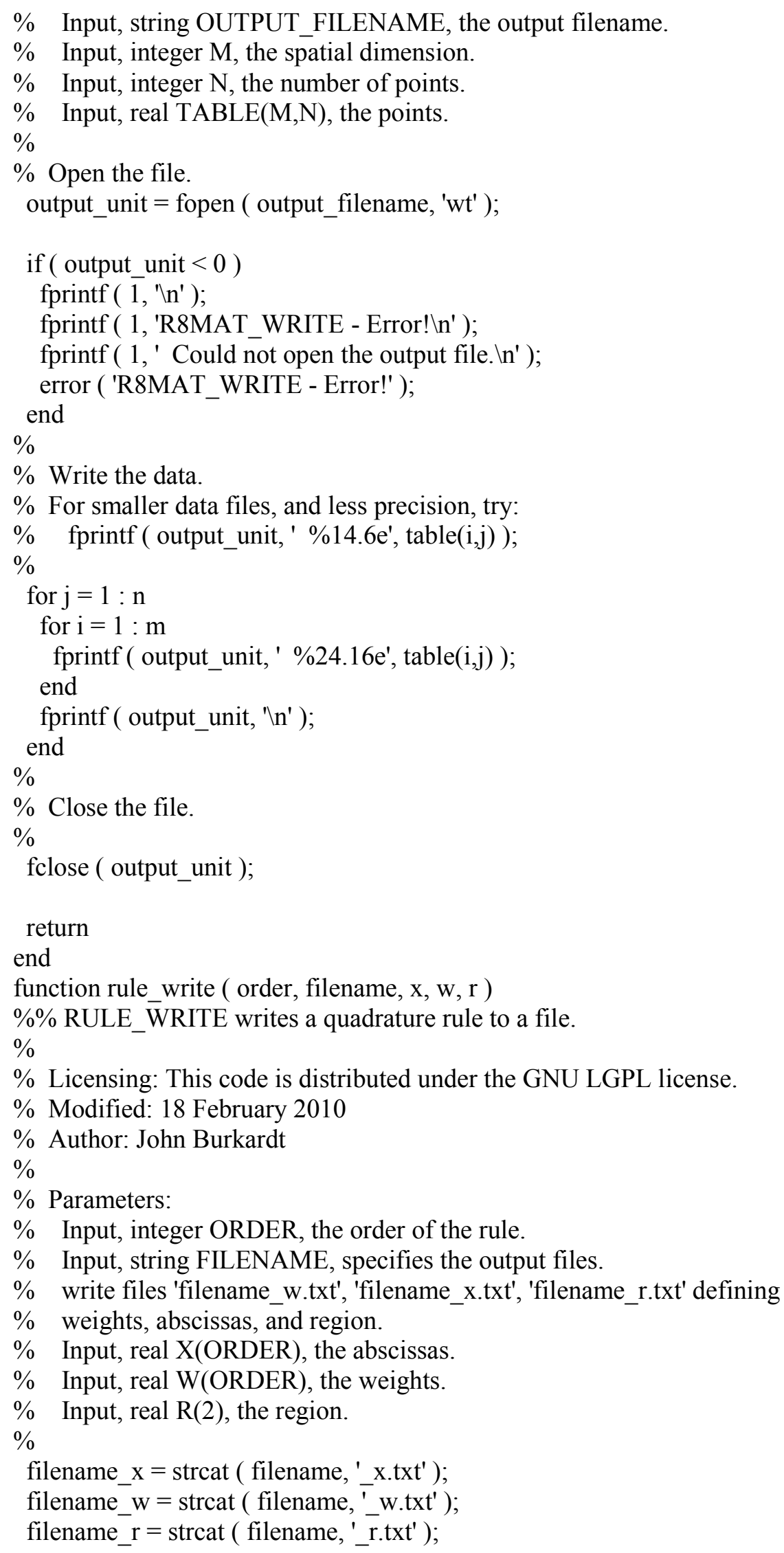




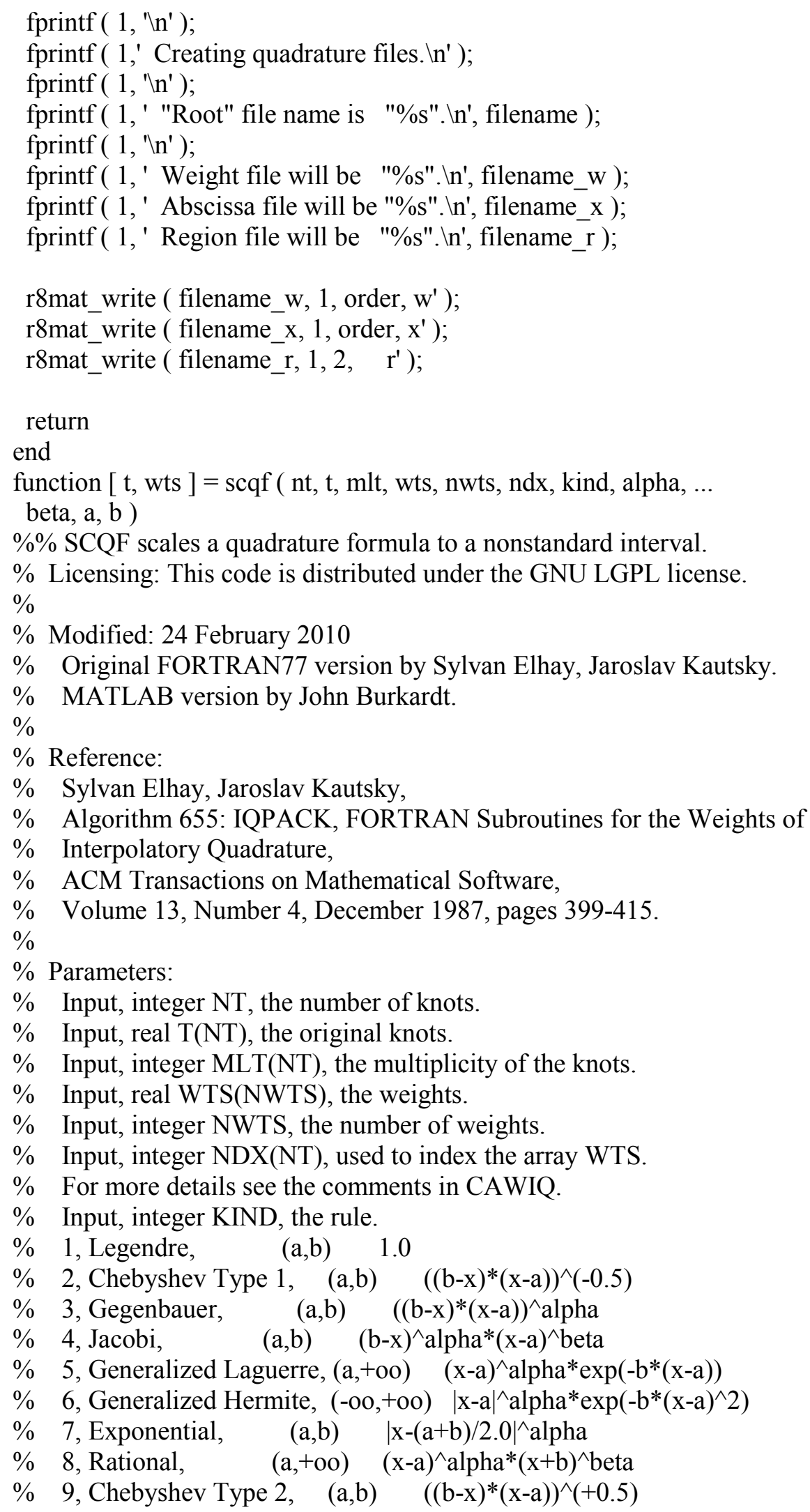


$\%$ Input, real ALPHA, the value of Alpha, if needed.

$\%$ Input, real BETA, the value of Beta, if needed.

$\%$ Input, real $\mathrm{A}, \mathrm{B}$, the interval endpoints.

$\% \quad$ Output, real T(NT), the scaled knots.

$\%$ Output, real WTS(NWTS), the scaled weights.

$\%$

temp = eps;

parchk ( kind, 1, alpha, beta )

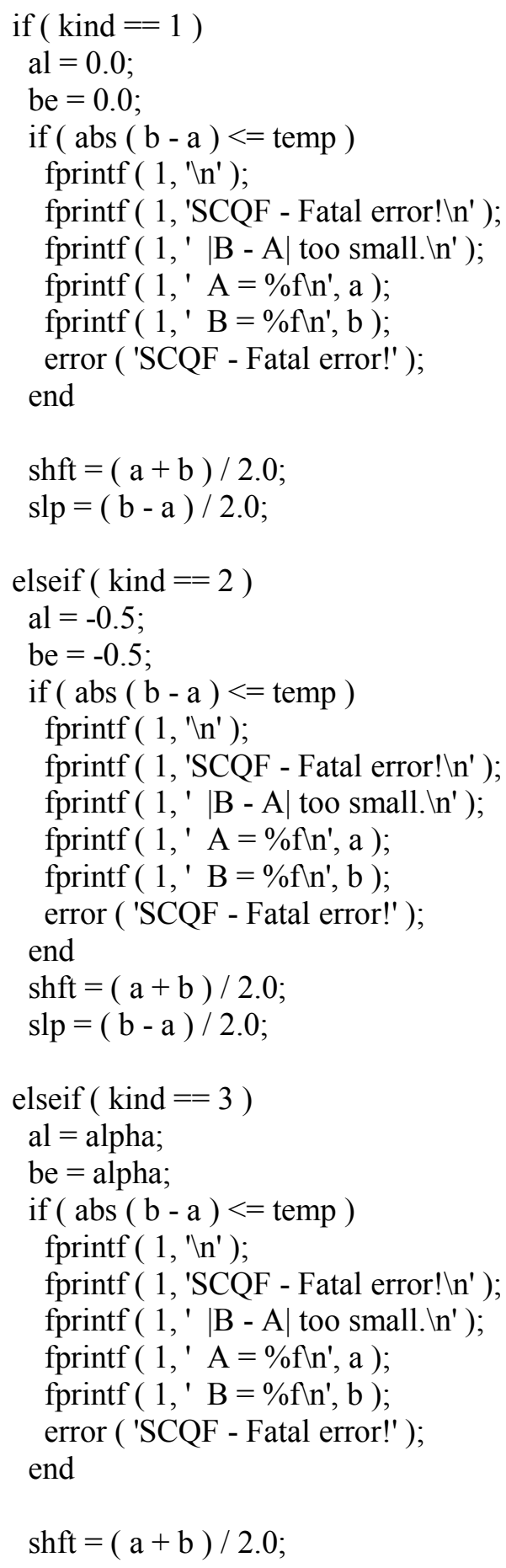




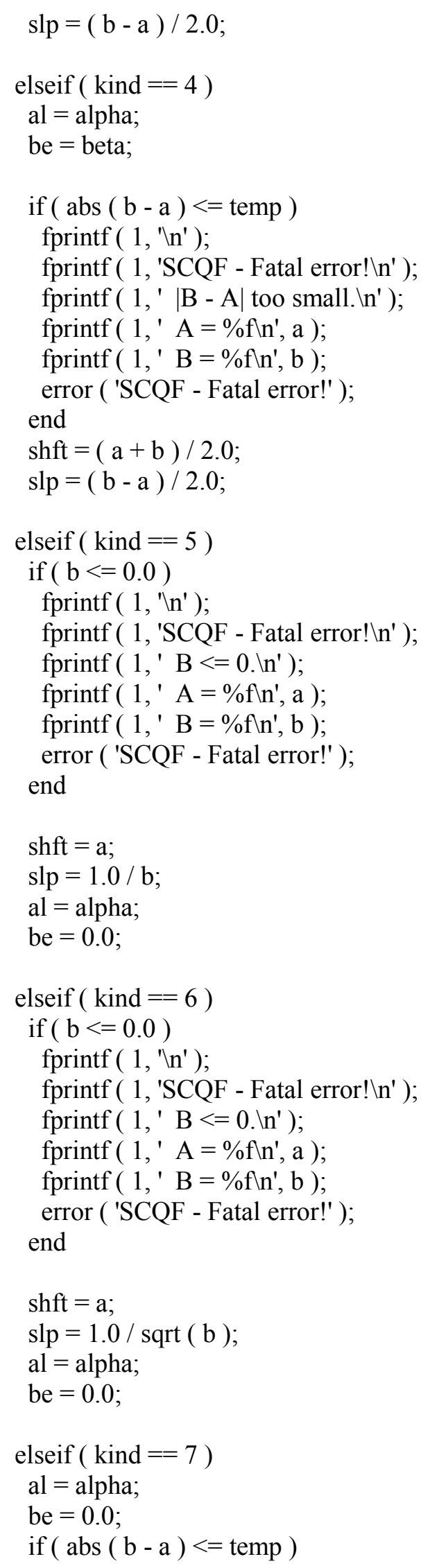




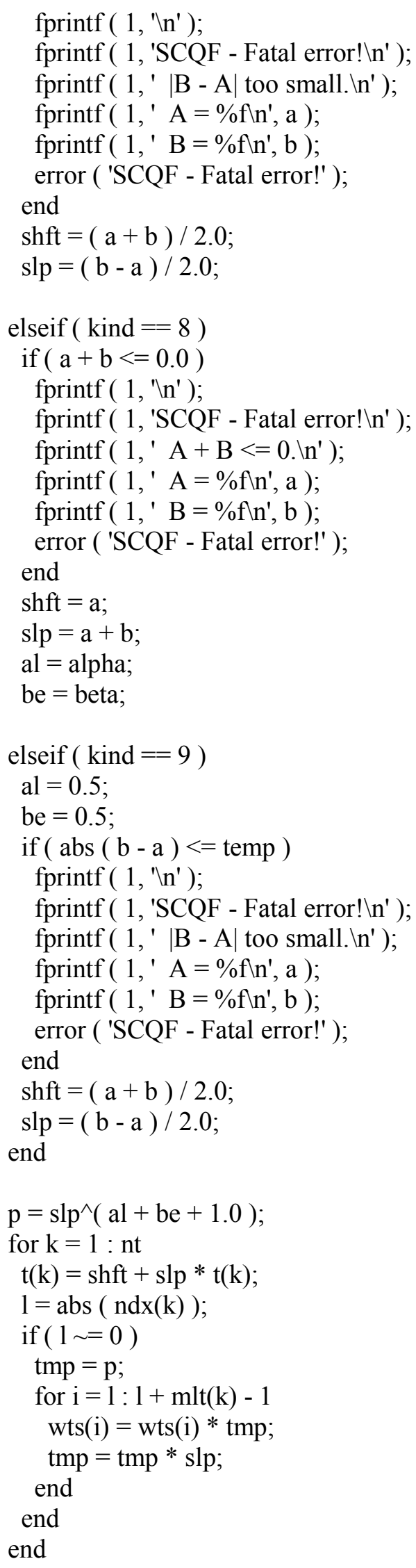


return

end

function [ $\mathrm{t}$, wts ] $=\operatorname{sgqf}(\mathrm{nt}, \mathrm{aj}, \mathrm{bj}, \mathrm{zemu})$

$\% \%$ SGQF computes knots and weights of a Gauss Quadrature formula.

$\%$

$\%$ Discussion:

$\%$ This routine computes all the knots and weights of a Gauss quadrature

$\%$ formula with simple knots from the Jacobi matrix and the zero-th

$\%$ moment of the weight function, using the Golub-Welsch technique.

$\%$

$\%$ Licensing: This code is distributed under the GNU LGPL license.

$\%$ Modified: 12 February 2010

$\%$ Original FORTRAN77 version by Sylvan Elhay, Jaroslav Kautsky.

$\% \quad$ MATLAB version by John Burkardt.

$\%$ Reference:

\% Sylvan Elhay, Jaroslav Kautsky,

$\%$ Algorithm 655: IQPACK, FORTRAN Subroutines for the Weights of

$\%$ Interpolatory Quadrature,

$\%$ ACM Transactions on Mathematical Software,

$\%$ Volume 13, Number 4, December 1987, pages 399-415.

$\%$

$\%$ Parameters:

$\%$ Input, integer NT, the number of knots.

$\%$ Input, real $\mathrm{AJ}(\mathrm{NT})$, the diagonal of the Jacobi matrix.

$\%$ Input, real BJ(NT), the subdiagonal of the Jacobi

$\%$ matrix, in entries 1 through NT-1. On output, BJ has been overwritten.

$\%$ Input, real ZEMU, the zero-th moment of the weight function.

$\%$ Output, real T(NT), the knots.

$\%$ Output, real WTS(NT), the weights.

$\%$

$\%$ Exit if the zero-th moment is not positive.

if $($ zemu $<=0.0)$

fprintf ( 1, ' 'n' );

fprintf ( 1, 'SGQF - Fatal error! \n' );

fprintf ( 1, ' ZEMU < =0. In' );

error ( 'SGQF - Fatal error!' );

end

$\%$

$\%$ Set up vectors for IMTQLX.

wts = zeros ( nt, 1$)$;

$\operatorname{wts}(1)=\operatorname{sqrt}($ zemu $)$;

wts $(2: \mathrm{nt})=0.0$;

$\%$

$\%$ Diagonalize the Jacobi matrix.

[ t, wts ] = imtqlx ( nt, aj, bj, wts );

$\operatorname{wts}(1: n t)=w t s(1: n t) .^{\wedge} 2$;

return

end 


\section{Reference}

[1] "The Third Great Wave (Special Report on The World Economy)," The Economists, London, UK, Oct-2014.

[2] D. Evans, "The Internet of Things: How the Next Evolution of Internet Is Changing Everything," 2011.

[3] G. P. Agrawal, Fiber-Optic Communication Systems, 4th ed. Wiley, 2010.

[4] J. P. Dunlop, Photovoltaic Systems, 3rd ed. Amer Technical Pub, 2012.

[5] K. E. Sapsford, C. Bradburne, J. B. Delehanty, and I. L. Medintz, "Sensors for detecting biological agents," Mater. Today, vol. 11, no. 3, pp. 38-49, Mar. 2008.

[6] J. R. Janesick, Scientific Charge-coupled Devices, 1st ed. SPIE Publications, 2001.

[7] T. M. Fortier, F. Quinlan, A. Hati, C. Nelson, J. A. Taylor, Y. Fu, J. Campbell, and S. A. Diddams, "Photonic microwave generation with high-power photodiodes.," Opt. Lett., vol. 38, no. 10, pp. 1712-4, May 2013.

[8] K. I. Sato, H. Hasegawa, T. Niwa, and T. Watanabe, "A large-scale wavelength routing optical switch for data center networks," IEEE Commun. Mag., vol. 51, no. 9, pp. 46-52, 2013.

[9] W. Shockley and H. J. Queisser, "Detailed Balance Limit of Efficiency of p-n Junction Solar Cells," J. Appl. Phys., vol. 32, no. 3, pp. 510-519, 1961.

[10] A. Shabaev, A. L. Efros, and A. J. Nozik, "Multiexciton generation by a single photon in nanocrystals," Nano Lett., vol. 6, no. 12, pp. 2856-2863, 2006.

[11] G. Masson, M. Latour, M. Rekinger, I.-T. Theologitis, M. Papoutsi, and C. Winneker, "Global Market Outlook for Photovoltaics 2013-2017," 2013.

[12] Solar Energy Industries Association, "Solar Market Insight Report 2013 Year in Review," 2013.

[13] U.S. Energy Information Administration, "How much electricity does a typical nuclear power plant generate," 2013. [Online]. Available:

http://www.eia.gov/tools/faqs/faq.cfm?id=104\&t=3. 
[14] International Renewable Energy Agency, "Solar Photovoltaics Technology Brief,” 2013.

[15] H. Hoppe and N. S. Sariciftci, "Organic solar cells: An overview," J. Mater. Res., vol. 19, no. 07, pp. 1924-1945, 2004.

[16] C. Deibel and V. Dyakonov, "Polymer fullerene bulk heterojunction solar cells," Rep. Prog. Phys., vol. 73, p. 96401, 2010.

[17] G. Yu, J. Gao, J. C. Hummelen, F. Wudl, and A. J. Heeger, "Polymer Photovoltaic Cells: Enhanced Efficiencies via a Network of Internal Donor-Acceptor Heterojunctions," Science (80-. )., vol. 270, no. 5243, pp. 1789-1791, 1995.

[18] M. A. Green, K. Emery, Y. Hishikawa, W. Warta, and E. D. Dunlop, "Solar cell efficiency tables (version 43)," Prog. Photovoltaics Res. Appl., vol. 22, no. 1, pp. 1-9, 2014.

[19] P. D. Cozzoli, T. Pellegrino, and L. Manna, "Synthesis, properties and perspectives of hybrid nanocrystal structures," Chem. Soc. Rev., vol. 35, no. 11, pp. 1195-1208, 2006.

[20] T. Xu and Q. Qiao, "Conjugated polymer-inorganic semiconductor hybrid solar cells," Energy Environ. Sci., vol. 4, no. 8, pp. 2700-2720, 2011.

[21] S. Dayal, N. Kopidakis, D. C. Olson, D. S. Ginley, and G. Rumbles, "Photovoltaic Devices with a Low Band Gap Polymer and CdSe Nanostructures Exceeding 3\% Efficiency," Nano Lett., vol. 10, no. 1, pp. 239-242, 2010.

[22] B. P. Nguyen, T. Kim, and C. R. Park, "Nanocomposite-Based Bulk Heterojunction Hybrid Solar Cells," J. Nanomater., vol. 2014, p. 243041, 2014.

[23] S. M. Sze and K. K. Ng, Physics of Semiconductor Devices. Wiley-Interscience, 2007.

[24] C. Honsberg and S. Bowden, "Standard Solar Spectra." [Online]. Available: http://pveducation.org/pvcdrom/appendices/standard-solar-spectra.

[25] NREL, "Reference Solar Spectral Irradiance: Air Mass 1.5." [Online]. Available: http://rredc.nrel.gov/solar/spectra/am1.5/.

[26] National Renewable Energy Laboratory, "Research Cell Efficiency Records,” 2014. [Online]. Available: http://www.nrel.gov/ncpv/.

[27] C. M. Natarajan, M. G. Tanner, and R. H. Hadfield, "Superconducting nanowire singlephoton detectors: physics and applications," Supercond. Sci. Technol., vol. 25, no. 6, p. 63001, 2012. 
[28] K. Kato, S. Hata, K. Kawano, and A. Kozen, "Design of Ultrawide-Band, HighSensitivity p-i-n Protodetectors (Special Issue on Optical/Microwave Interaction Devices, Circuits and Systems)," IEICE Trans. Electron., vol. 76, no. 2, pp. 214-221, Feb. 1993.

[29] C. H. Cox, Analog Optical Links: Theory and Practice, vol. 2006. Cambridge University Press, 2006, p. 302.

[30] C. H. Cox, E. I. Ackerman, G. E. Betts, and J. L. Prince, "Limits on the performance of RF-over-fiber links and their impact on device design," IEEE Trans. Microw. Theory Tech., vol. 54, no. 2, pp. 906-920, 2006.

[31] M. Faugeron, M. Chtioui, A. Enard, O. Parillaud, F. Lelarge, M. Achouche, J. Jacquet, A. Marceaux, and F. van Dijk, "High Optical Power, High Gain and High Dynamic Range Directly Modulated Optical Link," Light. Technol. J., vol. 31, no. 8, pp. 1227-1233, Apr. 2013.

[32] Z. Li, H. Pan, H. Chen, A. Beling, and J. C. Campbell, "High-saturation-current modified uni-traveling-carrier photodiode with cliff layer," IEEE J. Quantum Electron., vol. 46, no. 5, pp. 626-632, 2010.

[33] Y. Fu, "RF Characterization and Modeling of High-Power InGaAs/InP Photodiodes for Analog Optic Links," University of Virginia, 2013.

[34] C. B. . J. Norris and J. F. Gibbons, "Measurement of high-field carrier drift velocities in silicon by a time-of-flight technique," IEEE Trans. Electron Devices, vol. 14, no. 1, 1967.

[35] I. H. Campbell, D. L. Smith, C. J. Neef, and J. P. Ferraris, "Consistent time-of-flight mobility measurements and polymer light-emitting diode current-voltage characteristics," Appl. Phys. Lett., vol. 74, no. 19, p. 2809, 1999.

[36] A. M. Goodman and A. Rose, "Double Extraction of Uniformly Generated Electron-Hole Pairs from Insulators with Noninjecting Contacts," J. Appl. Phys., vol. 42, p. 2823, 1971.

[37] A. J. Campbell, D. D. C. Bradley, H. Antoniadis, M. Inbasekaran, W. S. W. Wu, and E. P. Woo, "Transient and steady-state space-charge-limited currents in polyfluorene copolymer diode structures with ohmic hole injecting contacts," Appl Phys Lett, vol. 76, no. 13 , pp. 1734-1736, 2000.

[38] M. A. Lampert, "Simplified theory of space-charge-limited currents in an insulator with traps," Phys. Rev., vol. 103, no. 6, pp. 1648-1656, 1956.

[39] J. M. Montero and J. Bisquert, "Interpretation of trap-limited mobility in space-charge limited current in organic layers with exponential density of traps," J. Appl. Phys., vol. 110, no. 4, 2011. 
[40] J. Lorrmann, B. H. Badada, O. Inganäs, V. Dyakonov, and C. Deibel, "Charge carrier extraction by linearly increasing voltage: Analytic framework and ambipolar transients," J. Appl. Phys., vol. 108, no. 11, 2010.

[41] E. Von Hauff, V. Dyakonov, and J. Parisi, "Study of field effect mobility in PCBM films and P3HT:PCBM blends," in Solar Energy Materials and Solar Cells, 2005, vol. 87, no. $1-4$, pp. 149-156.

[42] L. H. Jimison, M. F. Toney, I. McCulloch, M. Heeney, and A. Salleo, "Charge-Transport Anisotropy Due to Grain Boundaries in Directionally Crystallized Thin Films of Regioregular Poly(3-hexylthiophene)," Adv. Mater., vol. 21, no. 16, pp. 1568-1572, 2009.

[43] B.-Y. Chang and S.-M. Park, "Electrochemical impedance spectroscopy," Annu. Rev. Anal. Chem., vol. 3, pp. 207-229, 2010.

[44] G. Garcia-Belmonte, A. Munar, E. M. Barea, J. Bisquert, I. Ugarte, and R. Pacios, "Charge carrier mobility and lifetime of organic bulk heterojunctions analyzed by impedance spectroscopy," Org. Electron., vol. 9, no. 5, pp. 847-851, 2008.

[45] K. Li, Y. Shen, N. Majumdar, C. Hu, M. C. Gupta, and J. C. Campbell, "Determination of free polaron lifetime in organic bulk heterojunction solar cells by transient time analysis," J. Appl. Phys., vol. 108, no. 8, 2010.

[46] S. R. Cowan, R. A. Street, S. Cho, and A. J. Heeger, "Transient photoconductivity in polymer bulk heterojunction solar cells: Competition between sweep-out and recombination," Phys. Rev. B, vol. 83, no. 3, p. 35205, Jan. 2011.

[47] T. Tiedje and A. Rose, "A physical interpretation of dispersive transport in disordered semiconductors," Solid State Commun., vol. 37, no. 1, pp. 49-52, 1981.

[48] R. A. Street, K. W. Song, J. E. Northrup, and S. Cowan, "Photoconductivity measurements of the electronic structure of organic solar cells," Phys. Rev. B, vol. 83, no. 16, p. 165207, Apr. 2011.

[49] N. Christ, S. W. Kettlitz, S. Züfle, S. Valouch, and U. Lemmer, "Nanosecond response of organic solar cells and photodiodes: Role of trap states," Phys. Rev. B, vol. 83, no. 19, p. 195211, May 2011.

[50] B. Burkhart, P. P. Khlyabich, T. Cakir Canak, T. W. LaJoie, and B. C. Thompson, “'SemiRandom' Multichromophoric rr-P3HT Analogues for Solar Photon Harvesting," Macromolecules, vol. 44, no. 6, pp. 1242-1246, 2011.

[51] C. R. McNeill, I. Hwang, and N. C. Greenham, "Photocurrent transients in all-polymer solar cells: Trapping and detrapping effects," J. Appl. Phys., vol. 106, p. 24507, 2009. 
[52] V. D. Mihailetchi, H. X. Xie, B. de-Boer, L. J. A. Koster, and P. W. M. Blom, "Charge Transport and Photocurrent Generation in Poly(3-hexylthiophene): Methanofullerene Bulk-Heterojunction Solar Cells," Adv. Funct. Mater., vol. 16, no. 5, pp. 699-708, 2006.

[53] J. R. I. Lee, H. D. Whitley, R. W. Meulenberg, A. Wolcott, J. Z. Zhang, D. Prendergast, D. D. Lovingood, G. F. Strouse, T. Ogitsu, E. Schwegler, L. J. Terminello, and T. van Buuren, "Ligand-Mediated Modification of the Electronic Structure of CdSe Quantum Dots," Nano Lett., vol. 12, no. 6, pp. 2763-2767, 2012.

[54] S. Kilina, S. Ivanov, and S. Tretiak, "Effect of Surface Ligands on Optical and Electronic Spectra of Semiconductor Nanoclusters," J. Am. Chem. Soc., vol. 131, no. 22, pp. 77177726, 2009.

[55] P. Yang, T. Sergei, and I. Sergei, "Influence of Surfactants and Charges on CdSe Quantum Dots," J. Clust. Sci., vol. 22, no. 3, pp. 405-431, 2011.

[56] R. R. Lunt, T. P. Osedach, P. R. Brown, J. A. Rowehl, and V. Bulović, "Practical Roadmap and Limits to Nanostructured Photovoltaics," Adv. Mater., vol. 23, no. 48, pp. $5712-5727,2011$.

[57] M. D. Perez, C. Borek, S. R. Forrest, and M. E. Thompson, "Molecular and Morphological Influences on the Open Circuit Voltages of Organic Photovoltaic Devices," J. Am. Chem. Soc., vol. 131, no. 26, pp. 9281-9286, 2009.

[58] S. Kwon, H. C. Moon, K.-G. Lim, D. Bae, S. Jang, J. Shin, J. Park, T.-W. Lee, and J. K. Kim, "Improvement of power conversion efficiency of P3HT:CdSe hybrid solar cells by enhanced interconnection of CdSe nanorods via decomposable selenourea," J. Mater. Chem. A, vol. 1, no. 7, pp. 2401-2405, 2013.

[59] B. Sun and N. C. Greenham, "Improved efficiency of photovoltaics based on CdSe nanorods and poly(3-hexylthiophene) nanofibers," Phys. Chem. Chem. Phys., vol. 8, no. 30, pp. 3557-3560, 2006.

[60] J. D. Olson, G. P. Gray, and S. A. Carter, "Optimizing hybrid photovoltaics through annealing and ligand choice," Sol. Energy Mater. Sol. Cells, vol. 93, no. 4, pp. 519-523, Apr. 2009.

[61] K. Kumari, U. Kumar, S. N. Sharma, S. Chand, R. Kakkar, V. D. Vankar, and V. Kumar, "Effect of surface passivating ligand on structural and optoelectronic properties of polymer : CdSe quantum dot composites," Journal of Physics D: Applied Physics, vol. 41, no. 23. p. 235409, 2008.

[62] J. Y. Lek, G. Xing, T. C. Sum, and Y. M. Lam, "Electron transport limitation in P3HT:CdSe nanorods hybrid solar cells," ACS Appl. Mater. Interfaces, vol. 6, no. 2, pp. 894-902, 2014. 
[63] D. H. Webber and R. L. Brutchey, "Ligand Exchange on Colloidal CdSe Nanocrystals Using Thermally Labile tert-Butylthiol for Improved Photocurrent in Nanocrystal Films," J. Am. Chem. Soc., vol. 134, no. 2, pp. 1085-1092, 2012.

[64] M. J. Greaney, S. Das, D. H. Webber, S. E. Bradforth, and R. L. Brutchey, "Improving Open Circuit Potential in Hybrid P3HT:CdSe Bulk Heterojunction Solar Cells via Colloidal tert-Butylthiol Ligand Exchange," ACS Nano, vol. 6, no. 5, pp. 4222-4230, 2012.

[65] M. J. Greaney, J. Araujo, B. Burkhart, B. C. Thompson, and R. L. Brutchey, "Novel semirandom and alternating copolymer hybrid solar cells utilizing CdSe multipods as versatile acceptors," Chem. Commun., vol. 49, no. 77, pp. 8602-8604, 2013.

[66] D. V Lang, "Deep-level transient spectroscopy: A new method to characterize traps in semiconductors," J. Appl. Phys., vol. 45, no. 7, pp. 3023-3032, 1974.

[67] M. R. Burd and R. Braunstein, "Deep levels in semi-insulating liquid encapsulated Czochralski-grown GaAs," J. Phys. Chem. Solids, vol. 49, no. 7, pp. 731-735, 1988.

[68] C. Eiche, D. Maier, M. Schneider, D. Sinerius, J. Weese, K. W. Benz, and Honerkamp J, "Analysis of photoinduced current transient spectroscopy (PICTS) data by a regularisation method: Application of compensation defects in CdTe," J. Phys. Condens. Matter, vol. 4, pp. 6131-6140, 1992.

[69] P. Muret, J. Pernot, M. Azize, and Z. Bougrioua, "Photoinduced current transient spectroscopy of deep levels and transport mechanisms in iron-doped $\mathrm{GaN}$ thin films grown by low pressure-metalorganic vapor phase epitaxy," J. Appl. Phys., vol. 102, no. 5, p. 53701, 2007.

[70] S. Neugebauer, J. Rauh, C. Deibel, and V. Dyakonov, "Investigation of electronic trap states in organic photovoltaic materials by current-based deep level transient spectroscopy," Appl. Phys. Lett., vol. 100, no. 26, p. 263304, 2012.

[71] D. Schroder, Semiconductor Material and Device Characterization, 3rd ed. John Wiley \& Sons, Ltd., 2006.

[72] A. Miller and E. Abrahams, "Impurity Conduction at Low Concentrations," Phys. Rev., vol. 120, no. 3, pp. 745-755, Nov. 1960.

[73] R. Schmechel, "Hopping transport in doped organic semiconductors: A theoretical approach and its application to p-doped zinc-phthalocyanine," J. Appl. Phys., vol. 93, no. 8, pp. 4653-4660, 2003.

[74] R. C. Aster, B. Borchers, and C. H. Thurber, Parameter Estimation and Inverse Problems, 2nd ed. Academic Press, 2012. 
[75] P. C. Hansen, "Analysis of discrete ill-posed problems by means of the L-curve," SIAM Rev., vol. 34, no. 4, pp. 561-580, 1992.

[76] P. Hansen and D. O'Leary, "The Use of the L-Curve in the Regularization of Discrete IllPosed Problems," SIAM J. Sci. Comput., vol. 14, no. 6, pp. 1487-1503, 1993.

[77] R. C. I. MacKenzie, C. G. Shuttle, G. F. Dibb, N. Treat, E. von Hauff, M. J. Robb, C. J. Hawker, M. L. Chabinyc, and J. Nelson, "Interpreting the Density of States Extracted from Organic Solar Cells Using Transient Photocurrent Measurements," J. Phys. Chem. C, vol. 117, pp. 12407-12414, 2013.

[78] K. Li, L. Li, and J. C. Campbell, "Recombination lifetime of free polarons in polymer/fullerene bulk heterojunction solar cells," J. Appl. Phys., vol. 111, no. 3, p. 034503, 2012.

[79] M. Bertero, P. Brianzi, and E. R. Pike, "On the recovery and resolution of exponential relaxation rates from experimental data. III. The effect of sampling and truncation of data on the Laplace transform inversion," Proc. R. Soc. London. A. Math. Phys. Sci., vol. 398, no. 1814 , pp. 23-44, 1985.

[80] A. A. Istratov and O. F. Vyvenko, "Exponential analysis in physical phenomena," Rev. Sci. Instrum., vol. 70, no. 2, p. 1233, 1999.

[81] H. Bassler, "Charge Transport in Disordered Organic Photoconductors a Monte Carlo Simulation Study," Phys. Status Solidi, vol. 175, no. 1, pp. 15-56, 1993.

[82] Y. N. Gartstein and E. M. Conwell, "High-field hopping mobility in molecular systems with spatially correlated energetic disorder," Chem. Phys. Lett., vol. 245, no. 4-5, pp. 351-358, Nov. 1995.

[83] D. H. Dunlap, P. E. Parris, and V. M. Kenkre, "Charge-Dipole Model for the Universal Field Dependence of Mobilities in Molecularly Doped Polymers," Phys. Rev. Lett., vol. 77, no. 3, pp. 542-545, Jul. 1996.

[84] E. Lebedev, T. Dittrich, V. Petrova-Koch, S. Karg, and W. Brütting, "Charge carrier mobility in poly(p-phenylenevinylene) studied by the time-of-flight technique," Appl. Phys. Lett., vol. 71, no. 18, p. 2686, 1997.

[85] E. Couderc, N. Bruyant, A. Fiore, F. Chandezon, D. Djurado, P. Reiss, and J. FaureVincent, "Charge transport in poly(3-hexylthiophene):CdSe nanocrystals hybrid thin films investigated with time-of-flight measurements," Appl. Phys. Lett., vol. 101, no. 13, p. 133301, 2012.

[86] B. Huang, E. Glynos, B. Frieberg, H. Yang, and P. F. Green, "Effect of thicknessdependent microstructure on the out-of-plane hole mobility in poly(3-hexylthiophene) films," ACS Appl. Mater. Interfaces, vol. 4, no. 10, pp. 5204-5210, 2012. 
[87] W. Fu, Y. Shi, W. Qiu, L. Wang, Y. Nan, M. Shi, H. Li, and H. Chen, "High efficiency hybrid solar cells using post-deposition ligand exchange by monothiols.," Phys. Chem. Chem. Phys., vol. 14, no. 35, pp. 12094-8, 2012.

[88] K. Kumari, S. Chand, P. Kumar, S. N. Sharma, V. D. Vankar, and V. Kumar, "Effect of CdSe quantum dots on hole transport in poly(3-hexylthiophene) thin films," Appl. Phys. Lett., vol. 92, no. 26, p. 263504, 2008.

[89] V. Kažukauskas, E. Couderc, A. Sakavičius, A. Nekrošius, V. Vertelis, P. Reiss, D. Djurado, and J. Faure-Vincent, "Analysis of carrier transport in photovoltaic structures of P3HT with CdSe nanocrystals,” Appl. Surf. Sci., Sep. 2014.

[90] A. L. Domanski, I. Lieberwirth, E. Sengupta, K. Landfester, H. J. Butt, R. Berger, J. Rauh, V. Dyakonov, and C. Deibel, "Effect of morphological changes on presence of trap states in P3HT:PCBM solar cells studied by cross-sectional energy filtered TEM and thermally stimulated current measurements," J. Phys. Chem. C, vol. 117, no. 45, pp. 23495-23499, 2013.

[91] O. Oklobia and T. S. Shafai, "Correlation between charge carriers mobility and nanomorphology in a blend of P3HT/PCBM bulk heterojunction solar cell: Impact on recombination mechanisms," Sol. Energy Mater. Sol. Cells, vol. 122, pp. 158-163, Mar. 2014.

[92] R. C. I. MacKenzie, T. Kirchartz, G. F. A. Dibb, and J. Nelson, "Modeling Nongeminate Recombination in P3HT:PCBM Solar Cells," J. Phys. Chem. C, vol. 115, no. 19, pp. 9806-9813, 2011.

[93] J. Blakesley and D. Neher, "Relationship between energetic disorder and open-circuit voltage in bulk heterojunction organic solar cells," Phys. Rev. B, vol. 84, no. 7, 2011.

[94] R. C. I. MacKenzie, C. G. Shuttle, M. L. Chabinyc, and J. Nelson, "Extracting Microscopic Device Parameters from Transient Photocurrent Measurements of P3HT:PCBM Solar Cells," Adv. Energy Mater., vol. 2, no. 6, pp. 662-669, 2012.

[95] G. Gavazzi, G. and Sironi, "Cosmic-ray electrons and galactic radio noise: Some problems," La Riv. del Nuovo Cim., vol. 5, no. 2, pp. 155-186, 1975.

[96] S. Bonnefoy-Claudet, F. Cotton, and P.-Y. Bard, "The nature of noise wavefield and its applications for site effects studies," Earth-Science Rev., vol. 79, no. 3-4, pp. 205-227, Dec. 2006.

[97] A. A. Clerk, M. H. Devoret, S. M. Girvin, F. Marquardt, and R. J. Schoelkopf, "Introduction to quantum noise, measurement, and amplification," Rev. Mod. Phys., vol. 82, no. 2, pp. 1155-1208, Apr. 2010. 
[98] J. B. Johnson, "Thermal Agitation of Electricity in Conductors," Phys. Rev., vol. 32, no. 1, pp. 97-109, Jul. 1928.

[99] H. Nyquist, "Thermal Agitation of Electric Charge in Conductors," Phys. Rev., vol. 32, no. 1, pp. 110-113, Jul. 1928.

[100] A. Konczakowska and B. M. Wilamowski, "Noise in Semiconductor Devices," in Fundamentals of Industrial Electronics, 1st ed., CRC Press, 2011.

[101] B. J. Gross and C. G. Sodini, "1/f noise in MOSFETs with ultrathin gate dielectrics," in Electron Devices Meeting, 1992. IEDM '92. Technical Digest., International, 1992, pp. 881-884.

[102] L. M. Ward and P. E. Greenwood, “1/f noise,” Scholarpedia. p. 1537, 2007.

[103] A. L. McWhorter, "1/f noise and related surface effects in germanium.," MIT Lincoln Laboratory, Lexington, MA, 1955.

[104] A. L. McWhorter, "1/f noise and germanium surface properties," Semicond. Surf. Phys., pp. 207-228, 1957.

[105] K. K. Hung, P. K. Ko, C. Hu, and Y. C. Cheng, "A unified model for the flicker noise in metal-oxide-semiconductor field-effect transistors," Electron Devices, IEEE Trans., vol. 37, no. 3, pp. 654-665, 1990.

[106] F. N. Hooge, “1/f noise,” Phys. B+ C, vol. 83, no. 1, pp. 14-23, 1976.

[107] F. N. Hooge, “1/f noise sources,” Electron Devices, IEEE Trans., vol. 41, no. 11, pp. 1926-1935, 1994.

[108] T. G. M. Kleinpenning, “1/f Noise in pn diodes,” Phys. B+ C, vol. 98, no. 4, pp. 289-299, 1980.

[109] T. G. M. Kleinpenning, “1/f noise in p-n junction diodes,” J. Vac. Sci. Technol. A, vol. 3, pp. 176-182, 1985.

[110] F. N. Hooge, T. G. M. Kleinpenning, and L. K. J. Vandamme, "Experimental studies on 1/f noise," Reports Prog. Phys., vol. 44, no. 5, pp. 479-532, 1981.

[111] T. G. M. Kleinpenning, F. Schurink, and J. H. C. Van Der Veer, "1/f noise as a sensitive parameter in the life prediction testing of photovoltaic modules," Sol. cells, vol. 12, no. 4, pp. 363-370, 1984.

[112] L. K. J. Vandamme, "Noise as a diagnostic tool for quality and reliability of electronic devices," IEEE Trans. Electron Devices, vol. 41, pp. 2176-2187, 1994. 
[113] B. K. Jones, "Low-frequency noise spectroscopy," Electron Devices, IEEE Trans., vol. 41, no. 11, pp. 2188-2197, Nov. 1994.

[114] A. K. Raychaudhuri, "Measurement of $1 / \mathrm{f}$ noise and its application in materials science," Curr. Opin. solid state Mater. Sci., vol. 6, no. 1, pp. 67-85, 2002.

[115] S. L. Rumyantsev, M. S. Shur, M. E. Levinshtein, A. Motayed, and A. V. Davydov, "Low-frequency noise in GaN nanowire transistors," J. Appl. Phys., vol. 103, p. 064501, 2008.

[116] C. J. Delker, Y. Zi, C. Yang, and D. B. Janes, "Low-Frequency Noise Contributions from Channel and Contacts in InAs Nanowire Transistors," Electron Devices, IEEE Trans., vol. 60, pp. 2900-2905, 2013.

[117] A. A. Balandin, "Low-frequency 1/f noise in graphene devices.," Nat. Nanotechnol., vol. 8, pp. 549-555, 2013.

[118] L. Ke, X. Y. Zhao, R. S. Kumar, and S. J. Chua, "Low-frequency noise measurement and analysis in organic light-emitting diodes," Electron Device Lett. IEEE, vol. 27, no. 7, pp. 555-557, 2006.

[119] L. Ke, S. Bin Dolmanan, L. Shen, C. Vijila, S. J. Chua, R.-Q. Png, P.-J. Chia, L.-L. Chua, and P. K.-H. Ho, "Low frequency noise analysis on organic thin film transistors," J. Appl. Phys., vol. 104, no. 12, p. 124502, 2008.

[120] H. Kang, L. Jagannathan, and V. Subramanian, "Measurement, analysis, and modeling of 1/f noise in pentacene thin film transistors," Appl. Phys. Lett., vol. 99, no. 6, p. 62106, 2011.

[121] M. Bag, N. S. Vidhyadhiraja, and K. S. Narayan, "Fluctuations in photocurrent of bulk heterojunction polymer solar cells-A valuable tool to understand microscopic and degradation processes," Appl. Phys. Lett., vol. 101, no. 4, p. 043903, 2012.

[122] G. Landi, C. Barone, A. De Sio, S. Pagano, and H. C. Neitzert, "Characterization of polymer:fullerene solar cells by low-frequency noise spectroscopy," Appl. Phys. Lett., vol. 102, no. 22, p. 223902, 2013.

[123] B. Kippelen and J. Bredas, “Organic photovoltaics," Energy Environ. Sci., vol. 2, no. 3, pp. 251-261, 2009.

[124] K. Li, P. P. Khlyabich, L. Li, B. Burkhart, B. C. Thompson, and J. C. Campbell, "Influence of Exciton Diffusion and Charge-Transfer State Dissociation Efficiency on the Short-Circuit Current Densities in Semi-Random Donor/Acceptor Polymer:Fullerene Solar Cells," J. Phys. Chem. C, vol. 117, no. 14, pp. 6940-6948, 2013. 
[125] A. Pivrikas, N. S. Sariciftci, G. Juška, and R. Österbacka, "A review of charge transport and recombination in polymer/fullerene organic solar cells," Prog. Photovoltaics Res. Appl., vol. 15, no. 8, pp. 677-696, Dec. 2007.

[126] G. Li, V. Shrotriya, Y. Yao, and Y. Yang, "Investigation of annealing effects and film thickness dependence of polymer solar cells based on poly(3-hexylthiophene)," J. Appl. Phys., vol. 98, no. 4, p. 043704, 2005.

[127] X. Yang and A. Uddin, "Effect of thermal annealing on P3HT:PCBM bulk-heterojunction organic solar cells: A critical review," Renew. Sustain. Energy Rev., vol. 30, pp. 324-336, Feb. 2014.

[128] P. D. Welch, "The Use of Fast Fourier Transform for the Estimation of Power Spectra: A Method Based on Time Aver. aging Over Short, Modified Periodograms," IEEE Trans. AUDIO Electroacoust., vol. AU-15, pp. 70-73, 1967.

[129] D. G. Proakis, J.G. and Manolakis, Digital Signal Processing, 4th ed. Prentice Hall, 2006.

[130] S. Dongaonkar, J. D. Servaites, G. M. Ford, S. Loser, J. Moore, R. M. Gelfand, H. Mohseni, H. W. Hillhouse, R. Agrawal, M. A. Ratner, T. J. Marks, M. S. Lundstrom, and M. A. Alam, "Universality of non-Ohmic shunt leakage in thin-film solar cells," J. Appl. Phys., vol. 108, no. 12, p. 124509, 2010.

[131] P. R. F. Rocha, H. L. Gomes, L. K. J. Vandamme, D. M. De Leeuw, S. C. J. Meskers, and P. van de Weijer, "Low-frequency noise as a diagnostic tool for OLED reliability," in Noise and Fluctuations (ICNF), 2013 22nd International Conference on, 2013, pp. 1-4.

[132] D. V. Kuksenkov, H. Temkin, A. Osinsky, R. Gaska, and M. A. Khan, "Low-frequency noise and performance of GaN p-n junction photodetectors," J. Appl. Phys., vol. 83, no. 4, p. 2142, 1998.

[133] S. T. Hsu, "Surface-state related 1/f noise in p-n junctions and MOS transistors," Appl. Phys. Lett., vol. 12, no. 9, p. 287, 1968.

[134] P. Kolev, M. J. Deen, H. C. Liu, J. M. Li, M. Buchanan, and Z. R. Wasilewski, "Asymmetry in the dark current low frequency noise characteristics of B-B and B-C quantum well infrared photodetectors from 10 to $80 \mathrm{~K}$," J. Appl. Phys., vol. 87, no. 5, p. $2400,2000$.

[135] K. Li, L. Li, P. P. Khlyabich, B. Burkhart, W. Sun, Z. Lu, B. C. Thompson, and J. C. Campbell, "Breakdown mechanisms and reverse current-voltage characteristics of organic bulk heterojunction solar cells and photodetectors," J. Appl. Phys., vol. 115, p. 223104, Jun. 2014. 
[136] Q. K. Yang, F. Fuchs, J. Schmitz, and W. Pletschen, "Investigation of trap-assisted tunneling current in InAs/(GaIn)Sb superlattice long-wavelength photodiodes," Appl. Phys. Lett., vol. 81, no. 25, p. 4757, 2002.

[137] B. Chen, J. Yuan, and A. L. Holmes, "Dark current modeling of InP based SWIR and MWIR InGaAs/GaAsSb type-II MQW photodiodes," Opt. Quantum Electron., vol. 45, no. 3, pp. 271-277, 2013.

[138] T. G. M. Kleinpenning, "On low-frequency noise in tunnel diodes," Solid. State. Electron., vol. 21, no. 7, pp. 927-931, Jul. 1978.

[139] Y. Nemirovsky, "Tunneling and 1/f noise currents in HgCdTe photodiodes," J. Vac. Sci. Technol. B Microelectron. Nanom. Struct., vol. 10, no. 4, p. 1602, Jul. 1992.

[140] G. B. Alers, K. S. Krisch, D. Monroe, B. E. Weir, and A. M. Chang, "Tunneling current noise in thin gate oxides," Appl. Phys. Lett., vol. 69, no. 19, p. 2885, 1996.

[141] C. Deibel, A. Baumann, and V. Dyakonov, "Polaron recombination in pristine and annealed bulk heterojunction solar cells," Appl. Phys. Lett., vol. 93, no. 16, p. 163303, 2008 .

[142] A. van der Ziel, P. H. Handel, X. Zhu, and K. H. Duh, "A theory of the Hooge parameters of solid-state devices," Electron Devices, IEEE Trans., vol. 32, no. 3, pp. 667-671, Mar. 1985 .

[143] L. Li, K. Li, P. P. Khlyabich, B. Burkhart, B. C. Thompson, and J. C. Campbell, "Photocurrent transients in polymer-fullerene bulk heterojunction organic solar cells," in Photovoltaic Specialists Conference (PVSC), 2012 38th IEEE, 2012, pp. 2756-2760.

[144] B. C. O'Regan, K. Bakker, J. Kroeze, H. Smit, P. Sommeling, and J. R. Durrant, "Measuring Charge Transport from Transient Photovoltage Rise Times. A New Tool To Investigate Electron Transport in Nanoparticle Films," J. Phys. Chem. B, vol. 110, pp. 17155-17160, 2006.

[145] C. G. Shuttle, B. O’Regan, A. M. Ballantyne, J. Nelson, D. D. C. Bradley, J. de Mello, and J. R. Durrant, "Experimental determination of the rate law for charge carrier decay in a polythiophene: Fullerene solar cell," Appl. Phys. Lett., vol. 92, no. 9, p. 093311, 2008.

[146] G. Garcia-Belmonte, P. P. Boix, J. Bisquert, M. Sessolo, and H. J. Bolink, "Simultaneous determination of carrier lifetime and electron density-of-states in P3HT:PCBM organic solar cells under illumination by impedance spectroscopy," Sol. Energy Mater. Sol. Cells, vol. 94, no. 2, pp. 366-375, Feb. 2010.

[147] R. D. Pensack, K. M. Banyas, and J. B. Asbury, "Charge Trapping in Organic Photovoltaic Materials Examined with Time-Resolved Vibrational Spectroscopy," $J$. Phys. Chem. C, vol. 114, pp. 5344-5350, 2010. 
[148] Z. Li, “High-Power Photodiodes,” University of Virginia, 2011.

[149] T. M. Fortier, M. S. Kirchner, F. Quinlan, J. Taylor, J. C. Bergquist, T. Rosenband, N. Lemke, A. Ludlow, Y. Jiang, C. W. Oates, and S. A. Diddams, "Generation of ultrastable microwaves via optical frequency division," Nat. Photonics, vol. 5, no. 7, pp. 425-429, 2011.

[150] E. Starikov, "Upconversion as a Universal Mechanism of Noise Transformation," in AIP Conference Proceedings, 2005, vol. 800, pp. 460-465.

[151] S. Pérez, T. González, S. L. Delage, and J. Obregon, "Monte Carlo analysis of the influence of $\mathrm{dc}$ conditions on the upconversion of generation-recombination noise in semiconductors," Semiconductor Science and Technology, vol. 16, no. 2. pp. L8-L11, 2001.

[152] F. L. and F.-P. E. S. and J. S. R. Walls, "Origin of 1/f PM and AM noise in bipolar junction transistor amplifiers," Ultrason. Ferroelectr. Freq. Control. IEEE Trans., vol. 44, no. 2, pp. 326-334, 1997.

[153] M. Rudolph, F. Lenk, O. Llopis, and W. Heinrich, "On the simulation of low-frequency noise upconversion in InGaP/GaAs HBTs," IEEE Trans. Microw. Theory Tech., vol. 54, no. 7, pp. 2954-2961, 2006.

[154] R. Boudot and E. Rubiola, "Phase noise in RF and microwave amplifiers," IEEE Trans. Ultrason. Ferroelectr. Freq. Control, vol. 59, no. 12, pp. 2613-2624, 2012.

[155] H. Jiang, J. Taylor, F. Quinlan, T. Fortier, and S. A. Diddams, "Noise Floor Reduction of an Er:Fiber Laser-Based Photonic Microwave Generator,” Photonics Journal, IEEE, vol. 3, no. 6, pp. 1004-1012, Dec. 2011.

[156] J. Krupka, "Contactless methods of conductivity and sheet resistance measurement for semiconductors, conductors and superconductors," Meas. Sci. Technol., vol. 24, no. 6, p. $062001,2013$.

[157] J. Hlávka, "New contactless method for lifetime measurement in semiconductor wafers," Rev. Sci. Instrum., vol. 54, no. 10, p. 1386, 1983.

[158] D. K. Schroder, "Contactless surface charge semiconductor characterization," in Materials Science and Engineering B: Solid-State Materials for Advanced Technology, 2002, vol. 91-92, pp. 196-210.

[159] V. Zevin, J. T. Suss, A. Zemel, and S. Rotter, "A new method for contactless conductivity measurement of a semiconductor layer," Solid State Commun., vol. 66, no. 5, pp. 553555, May 1988. 
[160] M. P. De Haas and J. M. Warman, "Photon-induced molecular charge separation studied by nanosecond time-resolved microwave conductivity," Chem. Phys., vol. 73, no. 1-2, pp. 35-53, Dec. 1982.

[161] J. E. Kroeze, T. J. Savenije, M. J. W. Vermeulen, and J. M. Warman, “Contactless determination of the photoconductivity action spectrum, exciton diffusion length, and charge separation efficiency in polythiophene-sensitized TiO2 bilayers," J. Phys. Chem. $B$, vol. 107, no. 31, pp. 7696-7705, 2003.

[162] R. K. Ahrenkiel and S. W. Johnston, "An advanced technique for measuring minoritycarrier parameters and defect properties of semiconductors," Mater. Sci. Eng. B, vol. 102, no. 1-3, pp. 161-172, Sep. 2003.

[163] D. Chen, A. Nakahara, D. Wei, D. Nordlund, and T. P. Russell, "P3HT/PCBM Bulk Heterojunction Organic Photovoltaics: Correlating Efficiency and Morphology," Nano Lett., vol. 11, no. 2, pp. 561-567, 2011.

[164] “Helical antenna,” Wikipedia. 2014.

[165] Q. Zhou, A. S. Cross, A. Beling, Y. Fu, Z. Lu, and J. C. Campbell, "High-Power V-Band InGaAs/InP Photodiodes," Photonics Technol. Lett. IEEE, vol. 25, no. 10, pp. 907-909, 2013.

[166] J. Taylor, S. Datta, A. Hati, C. Nelson, F. Quinlan, A. Joshi, and S. Diddams, "Characterization of Power-to-Phase Conversion in High-Speed P-I-N Photodiodes," Photonics Journal, IEEE, vol. 3, no. 1, pp. 140-151, Feb. 2011.

[167] G. Dicker, M. P. de Haas, L. D. A. Siebbeles, and J. M. Warman, "Electrodeless timeresolved microwave conductivity study of charge-carrier photogeneration in regioregular poly(3-hexylthiophene) thin films," Phys. Rev. B, vol. 70, no. 4, p. 45203, Jul. 2004.

[168] M. C. Fravventura, J. Hwang, J. W. A. Suijkerbuijk, P. Erk, L. D. A. Siebbeles, and T. J. Savenije, "Determination of Singlet Exciton Diffusion Length in Thin Evaporated C60 Films for Photovoltaics," J. Phys. Chem. Lett., vol. 3, no. 17, pp. 2367-2373, 2012.

[169] D. H. K. Murthy, M. Gao, M. J. W. Vermeulen, L. D. A. Siebbeles, and T. J. Savenije, "Mechanism of Mobile Charge Carrier Generation in Blends of Conjugated Polymers and Fullerenes: Significance of Charge Delocalization and Excess Free Energy," J. Phys. Chem. C, vol. 116, no. 16, pp. 9214-9220, 2012. 